\title{
International Union of Basic and Clinical Pharmacology. XCIX. Angiotensin Receptors: Interpreters of Pathophysiological Angiotensinergic Stimuli ${ }^{\mathbb{S}}$
}

Sadashiva S. Karnik, Hamiyet Unal, Jacqueline R. Kemp, Kalyan C. Tirupula, Satoru Eguchi, Patrick M. L. Vanderheyden, and Walter G. Thomas

Department of Molecular Cardiology, Lerner Research Institute of Cleveland Clinic, Cleveland, Ohio (S.S.K., H.U., J.R.K., K.C.T.); Cardiovascular Research Center, Temple University School of Medicine, Philadelphia, Pennsylvania (S.E.); Faculty of Sciences and Bioengineering Sciences, Vrije Universiteit Brussel, Brussels, Belgium (P.M.L.V.); and Department of General Physiology, School of Biomedical Sciences, The University of Queensland, Brisbane, Queensland, Australia (W.G.T.)

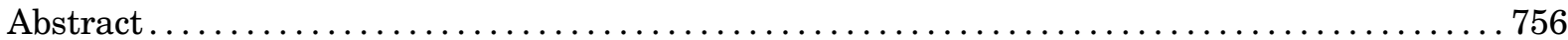

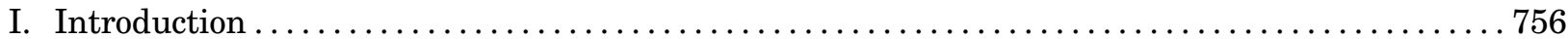

A. History, Classic Components, and Functions of Renin-Angiotensin System ........... 756

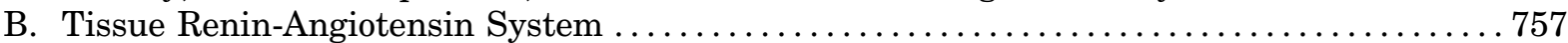

C. Intracellular Renin-Angiotensin System and Alternative Pathways Generating

Angiotensin Peptides .................................................. 757

D. Newer Components and Functional Axis of Renin-Angiotensin System . . . . . . . . . . . 758

E. Angiotensin Receptors Interpret Functions of Renin-Angiotensin System . ............. 759

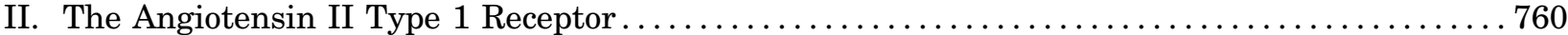

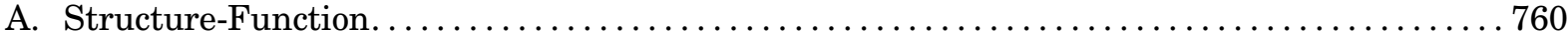

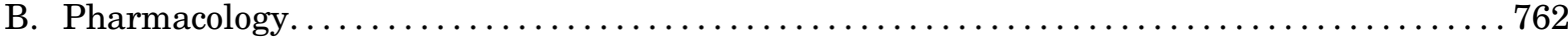

1. Pharmacophore Structure-Activity Relationship. ....................... 763

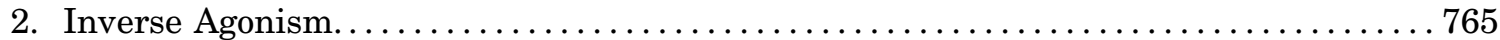

3. Insurmountable and Reversible Antagonism............................. 765

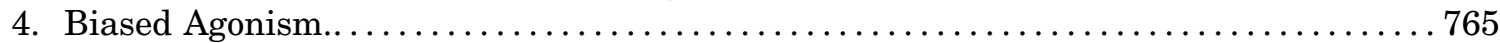

C. Mouse Models ........................................................ 766

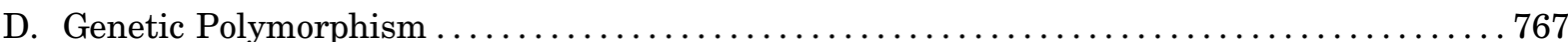

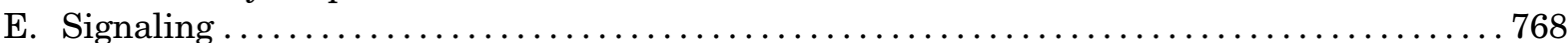

1. G-protein-Mediated Signaling................................... 768

2. G-Protein-Independent $\beta$-Arrestin-Mediated Signaling. . . . . . . . . . . . . . . . . . . 769

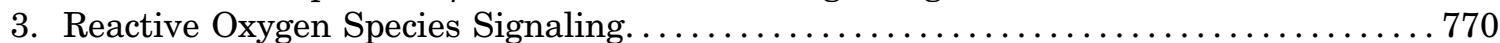

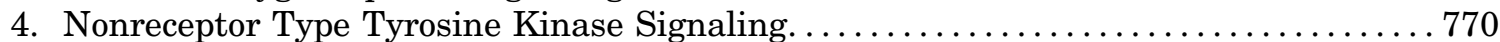

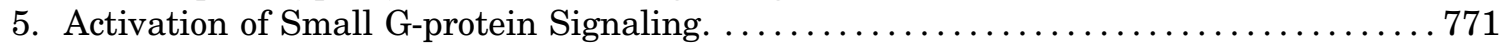

6. Transactivation of Receptor Tyrosine Kinase Signaling. ...................... 771

7. Signaling through $\mathrm{AT}_{1}$ Receptor Interacting Scaffold Proteins................. 771

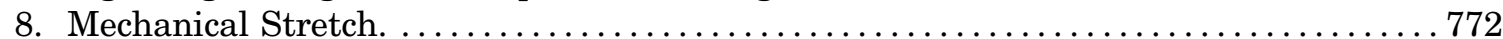

9. Signaling through Heterodimerization................................ 772

10. AngiotensinII Type 1 Receptor Signaling by Phosphorylation,

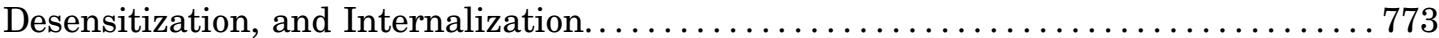

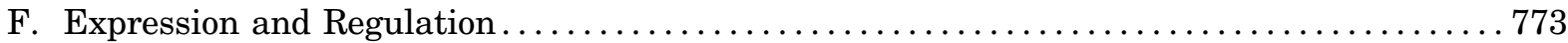

G. Pathophysiological Aspects of AngII Type 1 Receptor Activation ................. 774

This work was supported by National Institutes of Health National Heart, Lung, and Blood [Grants R01 HL57470 and HL115964 to S.S.K.]. S.S.K., H.U., J.J.K., and K.C.T. contributed equally to this work.

S.S.K., H.U., J.R.K., and K.C.T. are local members of Subcommittee on Angiotensin Receptor Nomenclaturel and S.E., P.M.L.V., and W.G. are external members.

Address correspondence to: Dr. Sadashiva S. Karnik, Chair, Subcommittee on Angiotensin Receptors, Nomenclature CommitteeInternational Union of Pharmacology, Department of Molecular Cardiology, Lerner Research Institute of Cleveland Clinic, 9500 Euclid Ave., NB5-76, Cleveland, OH 44195. E-mail: karniks@ccf.org

dx.doi.org/10.1124/pr.114.010454.

\$This article has supplemental material available at pharmrev.aspetjournals.org. 
1. Cardiovascular Remodeling and Hypertrophy........................... 774

2. Vascular Inflammation and Atherosclerosis............................... 775

3. Endothelial Dysfunction. ....................................... 775

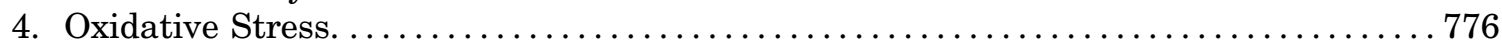

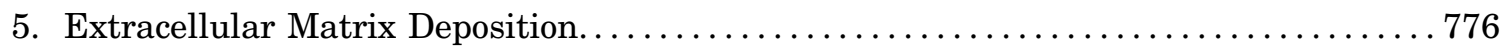

6. Insulin Resistance. ............................................. 776

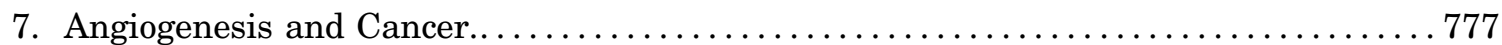

8. Autoantibodies and Malignant Hypertension. . . . . . . . . . . . . . . . . . . . . 777

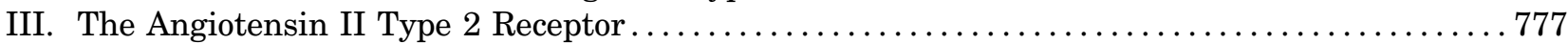

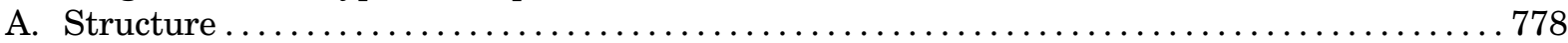

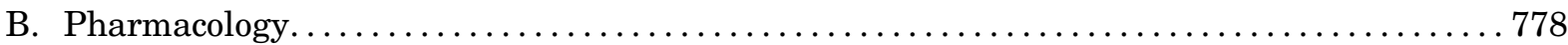

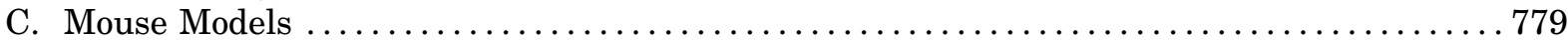

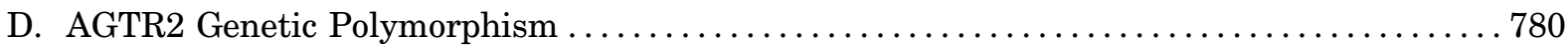

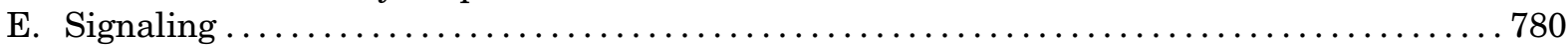

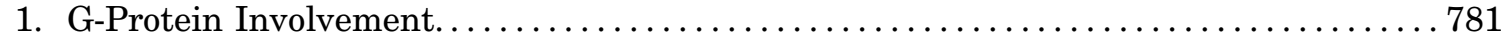

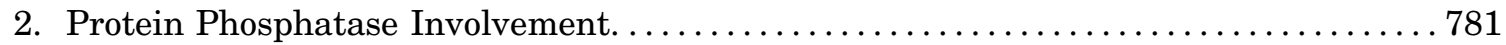

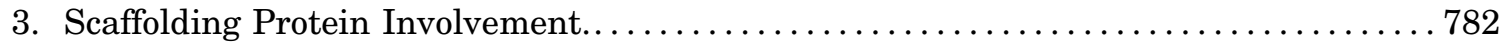

4. Nitric Oxide/cGMP Involvement...................................... 783

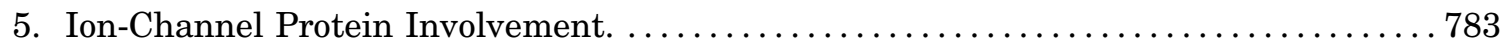

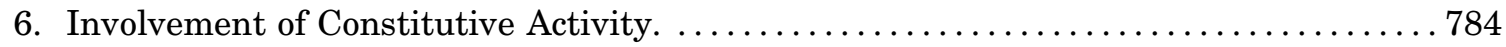

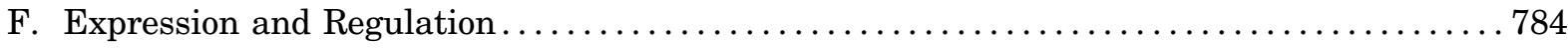

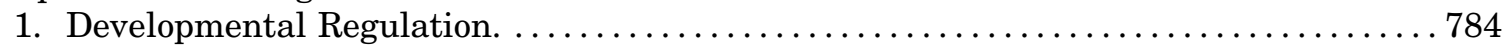

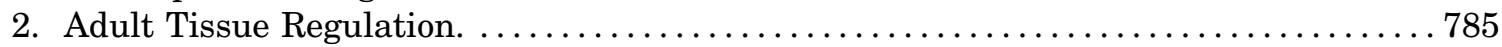

G. Pathophysiological Aspects of AngII Type 2 Receptor Activation . . . . . . . . . . . . . 785

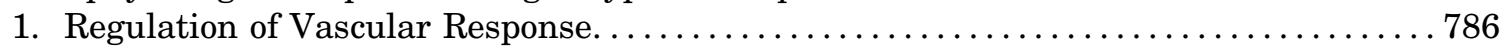

2. Regulation of Cardiac Growth Response............................. 786

3. Regulation of Fibrosis Response in Other Tissue.......................... 787

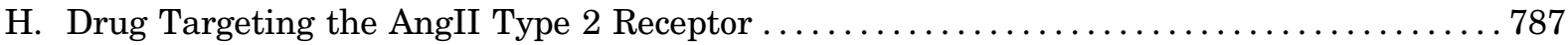

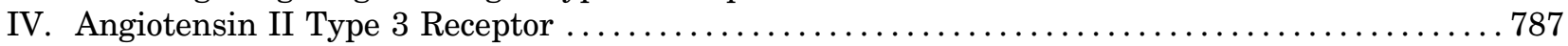

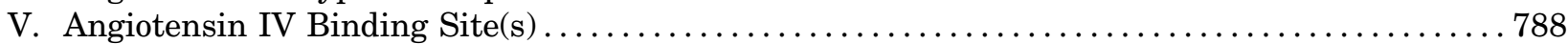

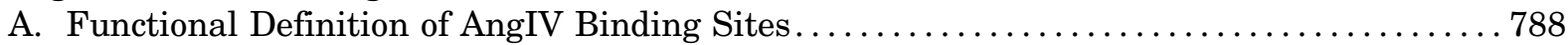

B. Structure of the AngIV Binding Protein, Insulin Regulated Amino Peptidase ........... 788

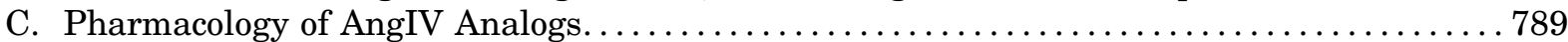

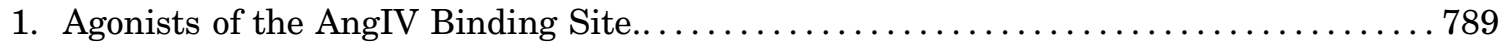

2. Antagonists of the AngIV Binding Site ............................... 790

3. Inhibitors of the Insulin Regulated Amino Peptidase Activity. . . . . . . . . . . . 790

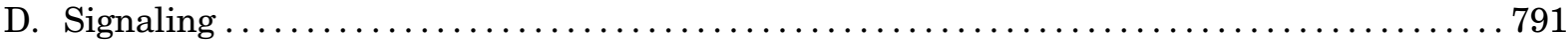

1. Effects on Insulin Regulated Amino Peptidase Signaling as a

Transmembrane Receptor....................................... 791

\begin{abstract}
ABBREVIATIONS: ACE, angiotensin converting enzyme; ADAM, A Disintegrin and Metalloproteinase; AGT, angiotensinogen; Ang(1-7), angiotensin (1-7, Asp $\left.{ }^{1}-\mathrm{Arg}^{2}-\mathrm{Val}^{3}-\mathrm{Tyr}^{4}-\mathrm{Ile}^{5}-\mathrm{His}^{6}-\mathrm{Pro}^{7}\right)$; Ang, angiotensin; AngII, octapeptide angiotensin $\left(\mathrm{Asp}^{1}-\mathrm{Arg}^{2}-\mathrm{Val}^{3}-\mathrm{Tyr}^{4}-\mathrm{Ile}^{5}-\mathrm{His}^{6}-\mathrm{Pro}^{7}-\right.$

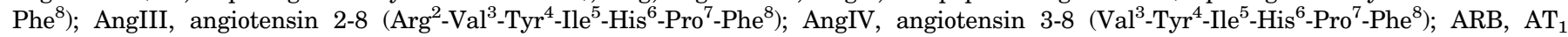
receptor blocker; $\mathrm{AT}_{1}$ receptor, AngII type 1 receptor; $\mathrm{AT}_{2}$ receptor, AngII type 2 receptor; AR234960, 1-[[(4S)-4-(3-fluorophenyl)-1-(2-methoxy4-nitrophenyl)sulfonylpyrrolidin-3-yl]methyl]-4-pyridin-2-ylpiperazine; AR244555, 1'-but-3-enyl-5-chlorospiro[2H-indole-3,4'-piperidine]-1-yl)(2,6-difluorophenyl)methanone; $\mathrm{AT}_{3}$ receptor, AngII type 3 receptor; ATN, antiretroviral toxic neuropathy; $\mathrm{ATRAP}$, $\mathrm{AT}_{1}$ receptor-associated protein; AVP, vasopressin; C21, Compound 21; CGP42112, nicotinic acid-Tyr- $N$-benzoxyl-carbonyl-Arg-Lys-His-Pro-Ile-OH; CHO, Chinese hamster ovary; CTGF, connective tissue growth factor; DAG, diacylglycerol; ECL, extracellular loop; eGFP, enhanced green fluorescent protein; EGFR, AT 1 receptorassociated protein (ATRAP1); EMA300, 5-[2,2-di(phenyl)acetyl]-4-[(4-methoxy-3-methylphenyl)methyl]-1,4,6,7-tetrahydroimidazo[4,5-c]pyridine-6carboxylic acid; EMA401, (S)-2-(diphenylacetyl)-1,2,3,4-tetrahydro-6-methoxy-5-(phenylmethoxy)-3-isoquinolinecarboxylic acid; ERK, extracellular signal-regulated kinase; 3-D, three-dimensional; GPCR, G-protein-coupled receptor; GRK, G-protein-coupled receptor kinase; HF, heart failure; HGF, hepatocyte growth factor; ICL3, intracellular third loop; IL, interleukin; IP3, inositol triphosphates; IRAP, insulin regulated amino peptidase, also AngIV binding site; IRS, insulin receptor substrates; L-158809, 2-ethyl-5,7-dimethyl-3-[[4-[2-(2H-tetrazol-5-yl)phenyl]phenyl]methyl]imidazo[5,4-b] pyridine; LDL, low-density lipoprotein; MAPK, mitogen-activated protein kinase; MAS, putative Ang(1-7) receptor, also product of the MAS oncogene; $\mathrm{NF}_{\kappa} \mathrm{B}$, nuclear factor; NOS, nitric oxide synthase; PD123177, trifluoroacetate salt; PD123319, ditrifluoroacetate; PDGFR, platelet derived growth factor receptor; PKC, protein kinase C; PLC, phospholipase C; PLZF, transcription factor promyelocytic zinc finger protein; PRR, prorenin receptor; PTZ, pentylenetetrazol; RAS, renin-angiotensin system; ROS, reactive oxygen species; RTK, receptor tyrosine kinase; SHP, Src homology phosphatase; SHR, spontaneously hypertensive rats; SNP, single nucleotide polymorphism; TM, transmembrane; VEGF, vascular endothelial growth factor; VIF, vasoconstriction-inhibiting factor; VSMC, vascular smooth muscle cells.
\end{abstract}


2. Effects on Insulin Regulated Amino Peptidase-Dependent

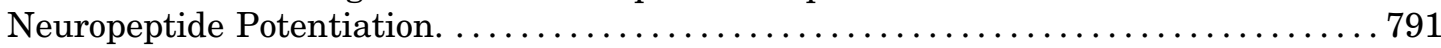

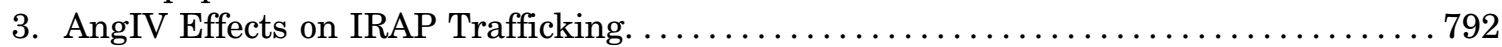

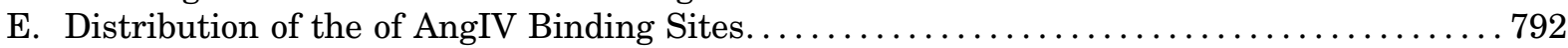

F. Insulin Regulated Amino Peptidase Gene Knockout Mice ....................... 793

1. Evaluation of AngIV-dependent Functions........................... 793

2. Nuances for Defining Receptor(s) for AngIV ........................... 794

3. AngIV Binding Site Conclusion..................................... 794

VI. Receptor Conundrum in the Renin-Angiotensin System . . . . . . . . . . . . . . . . . . 795

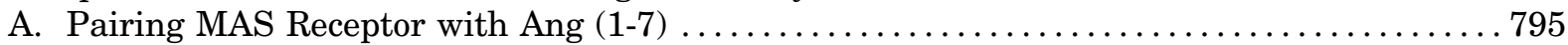

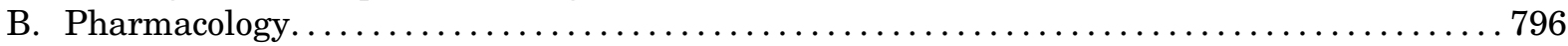

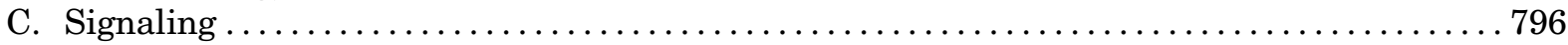

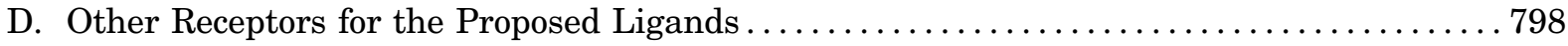

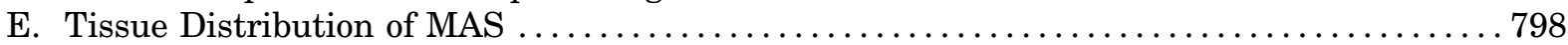

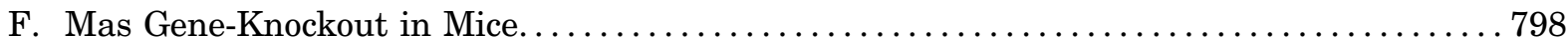

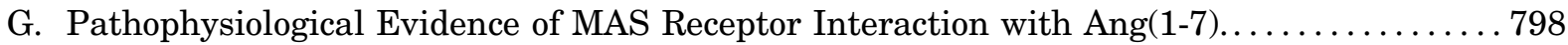

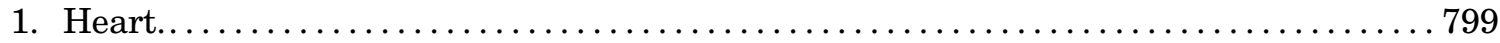

2. Kidney. .................................................... 799

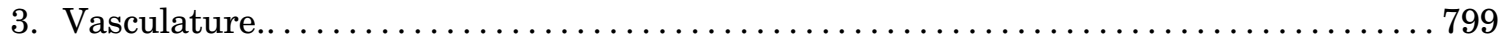

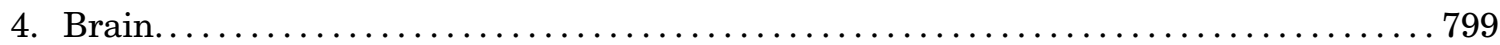

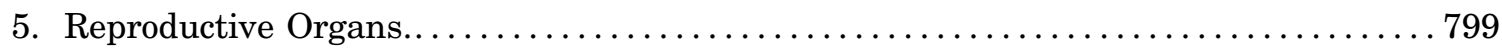

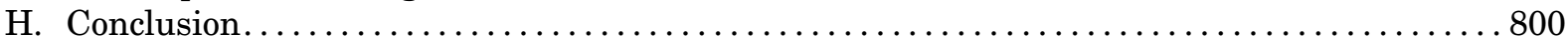

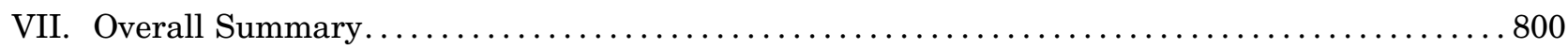

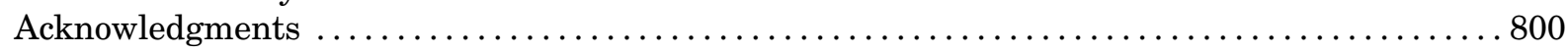

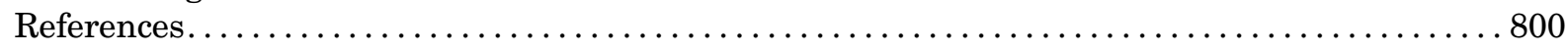

Abstract_— The renin angiotensin system (RAS) produced hormone peptides regulate many vital body functions. Dysfunctional signaling by receptors for RAS peptides leads to pathologic states. Nearly half of humanity today would likely benefit from modern drugs targeting these receptors. The receptors for RAS peptides consist of three G-protein-coupled receptorsthe angiotensin $\mathrm{II}$ type 1 receptor (AT $\mathrm{T}_{1}$ receptor), the angiotensin II type 2 receptor $\left(A_{2} T_{2}\right.$ receptor), the MAS receptor-and a type II trans-membrane zinc proteinthe candidate angiotensin IV receptor (AngIV binding site). The prorenin receptor is a relatively new contender for consideration, but is not included here because the role of prorenin receptor as an independent endocrine mediator is presently unclear. The full spectrum of biologic characteristics of these receptors is still evolving, but there is evidence establishing unique

\section{Introduction}

The angiotensin receptor field has featured an enormous development since the last IUPHAR review, with $>7255$ peer review publications and reviews. Many of these belong to various facets of the type 1 angiotensin receptor followed by the type 2 receptor literature, which is closely followed by the upstart newcomer MAS receptor literature (Fig. 1). We have tried to identify all key papers and year-by-year break down on each of the four angiotensin receptors by carrying out systematic searches, initially using broad search terms and finally narrowing down to specific receptors. We recognize that computer searches do not fully cover all aspects of a particular roles of each receptor in cardiovascular, hemodynamic, neurologic, renal, and endothelial functions, as well as in cell proliferation, survival, matrix-cell interaction, and inflammation. Therapeutic agents targeted to these receptors are either in active use in clinical intervention of major common diseases or under evaluation for repurposing in many other disorders. Broad-spectrum in fluence these receptors produce in complex pathophysiological context in our body highlights their role as precise interpreters of distinctive angiotensinergic peptide cues. This review article summarizes findings published in the last 15 years on the structure, pharmacology, signaling, physiology, and disease states related to angiotensin receptors. We also discuss the challenges the pharmacologist presently faces in formally accepting newer members as established angiotensin receptors and emphasize necessary future developments. receptor, but we have added selected references to more detailed reviews, which should be consulted for further information. To provide access to a wide range of specific information and the biologic activity data for each receptor detailed in this review, links are provided to IUPHAR/BPS receptor page for each receptor. Readers may also navigate to the IUPHAR/BPS Guide to Pharmacology website (www.guidetopharmacology.org).

\section{A. History, Classic Components, and Functions of Renin-Angiotensin System}

"Renin" was discovered in kidney extract as a hypertensive factor nearly 117 years ago by Tigerstedt and Bergman (1898). Hypertension in humans and 
A

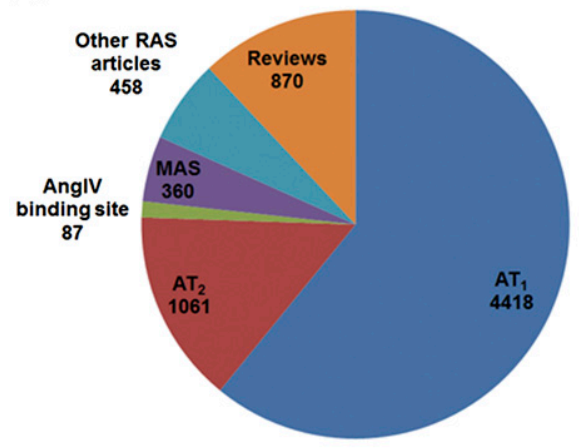

B

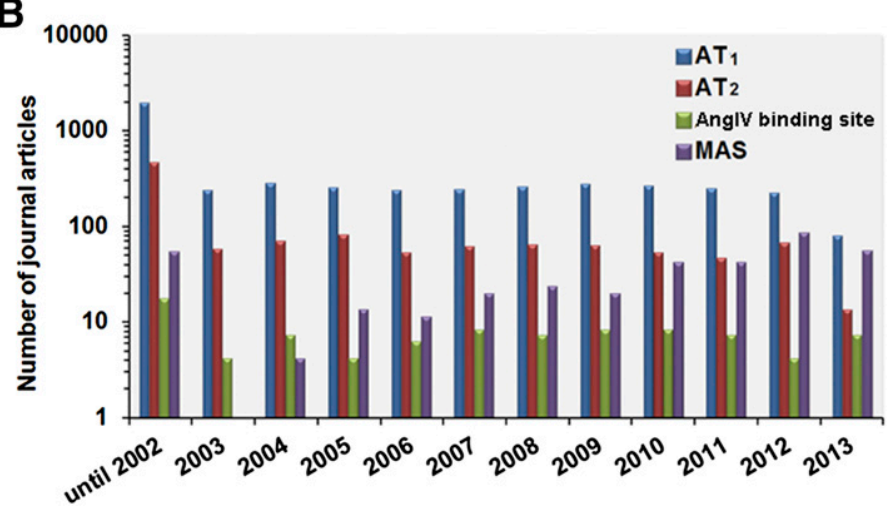

Fig. 1. Literature search and analysis of primary journal articles on RAS receptors. The journal articles published on RAS were automatically retrieved from PubMed with the search term "(((( RAS[Title/Abstract]) AND angiotensin, or $\mathrm{AT}_{1}$ receptor, or $\mathrm{AT}_{2}$ receptor, or $\mathrm{AT}_{3}$ receptor, or AngIV binding site, or MAS1[Title/Abstract]) NOT medication adherence scale[Title/Abstract])". The extracted references list had a total of 9147 reviews, opinions, commentary, lectures, and primary journal articles through the end of year 2013. The results were manually curated to retain relevant, nonredundant references. The primary journal articles were separated from the reviews, interviews, lectures, or commentary articles. The pool of these 7255 primary journal articles were further analyzed to illustrate number of publications under each RAS receptor as in the pie chart (A). The bar graph represents publication for each receptor/year (B). Python and Bio python scripts were used to aid in the literature search and analysis.

animal models was described as a renovascular disease (Goldblatt et al., 1934). Page and Helmer (1940) isolated angiotensinogen, which they named as "renin activator" at that time, and they proceeded to isolate a vasoconstrictor substance "angiotonin" in the blood from live animals infused with renin. An identical vasoactive compound identified in Goldblatt hypertensive dog ischemic kidney by Braun-Menendez was named "hypertensin." The independently isolated pressor substance was later shown to be an octapeptide and not its decapeptide precursor (Skeggs et al., 1956; Bumpus et al., 1957; Elliott and Peart, 1957). Now the octapeptide bears the hybrid name angiotensin II (AngII) in honor of the original independent discovery of this important endocrine hormone with numerous actions beyond its hypertensive effects. The precursor AngI generated by renin action on angiotensinogen has no vasopressor activity. The sequence of human AngII is $\mathrm{Asp}^{1}-\mathrm{Arg}^{2}-\mathrm{Val}^{3}-\mathrm{Tyr}^{4}-\mathrm{Ile}^{5}-\mathrm{His}^{6}-\mathrm{Pro}^{7}-\mathrm{Phe}^{8}$. The quest for a peptide antagonist drug to control hypertension began with establishment of bioactivity of total chemically synthesized AngII (Bumpus et al., 1957; Rittel et al., 1957), which lead to an era of establishing the structure-activity relationship of angiotensin analogs in tissues (Khosla et al., 1974; Meyer et al., 1974; Peach and Levens, 1980).

The cascade of proteolytic steps leading to the formation of AngII in vivo and components of the renin angiotensin system (RAS) were characterized in later years (Fig. 2). RAS maintains normal blood pressure in vivo by regulating fluid volume and the vascular structure as well as integrity. AngII regulates blood volume through water-electrolyte balance (content) and also modulates cardiac output, vascular resistance (container). RAS is activated in response to decreased plasma sodium level and fluid volume, which stimulate juxtaglomerular cells in the kidneys to secrete renin. The enzyme renin cleaves angiotensinogen (AGT) released to circulation by liver to the decapeptide angiotensin I (AngI) in circulation. The octapeptide AngII is produced by the carboxypeptidase angiotensin converting enzyme (ACE1) predominantly located on endothelial cells. This constitutes the classic circulating RAS, an elaborate endocrine system that stimulates adrenal gland to release aldosterone to regulate fluid volume/electrolytes and nerve ends to release catecholamine to regulate vascular tone. It is a multifunctional hormone influencing many cellular processes, including cell growth, apoptosis, migration, inflammation, and fibrosis (Hunyady, 2009; Stegbauer and Coffman, 2011; Horiuchi et al., 2012).

\section{B. Tissue Renin-Angiotensin System}

Apart from being a circulating hormone, locally produced AngII in most tissues functions as a paracrine and autocrine hormone (Dzau and Gibbons, 1987; Griffin et al., 1991; Weber et al., 1995). In this mode, the tissue RAS regulates long-term and chronic responses to locally produced AngII in tissues, including the brain, heart, kidneys, pancreas, vasculature, and adipose tissue. Importantly, tissue RAS functions independently of circulating RAS, providing critical paracrine or autocrine control in pathophysiological conditions, including hypertension, inflammation, thrombosis, atherosclerosis, diabetes, end-stage renal disease, coronary artery disease, cardiovascular hypertrophy, and heart failure (HF) (Lijnen and Petrov, 1999; Kim and Iwao, 2000; Lavoie and Sigmund, 2003; Mehta and Griendling, 2007).

\section{Intracellular Renin-Angiotensin System and Alternative Pathways Generating Angiotensin Peptides}

Cardiac and vascular smooth muscle cells and fibroblasts have been reported to harbor an intracellular AngII production pathway sometimes also referred as an "intracrine system" (Kumar et al., 2009). Often the 


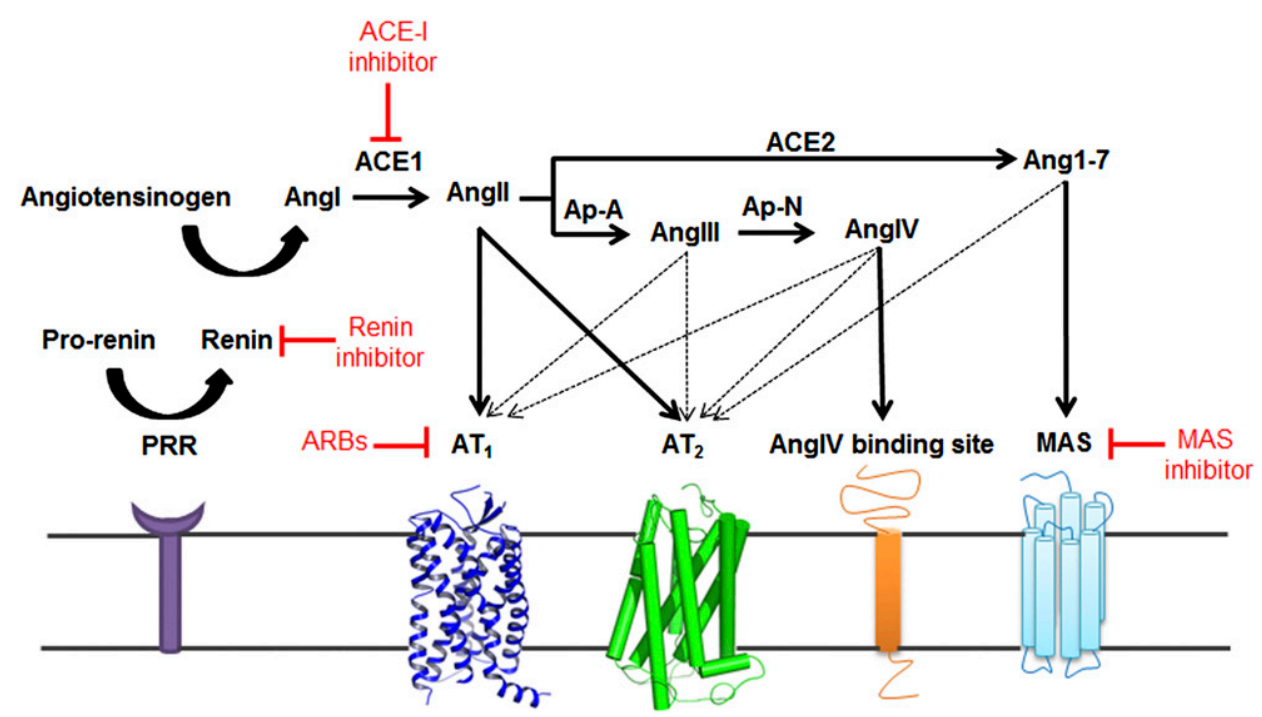

Fig. 2. A current view of enzymatic pathways generating hormonal peptides of RAS and corresponding cell surface receptors. Surrogate enzymes, such as mast cell chymase, neutral endopeptidase, tonin, cathepsin D, kalikrine, and aminopeptidases are also known to participate in angiotensin metabolism in different contexts. Some authors contend inclusion of these enzymes as components of RAS. Inhibitors of different components of RAS are shown in red.

major components of RAS, renin, AGT, and ACE do not coexist in most cells to fully support an intracellular RAS paradigm. However, alternative enzymes, such as cathepsins and chymases may produce functional AngII intracellularly (Kumar et al., 2008; Kumar and Boim, 2009). Depending on the cell type, cathepsin D can participate in the first step of intracellular AGT cleavage usually catalyzed by renin and the second step by chymase, not ACE (Wasse et al., 2012). Obviously, ACE inhibitors do not inhibit the intracellular RAS. Similarly, intracellular AngII actions are not prevented by Ang receptor blockers (Baker et al., 2004; Baker and Kumar, 2006; Singh et al., 2007). Importance of intracellular RAS concept in pathophysiology of disease is extensively described in several reports (Carey, 2012; Cook and Re, 2012; Ellis et al., 2012; Gwathmey et al., 2012; Kumar et al., 2012). However, controversy exists because it is recognized that extracellular AngII may be internalized by receptor-mediated processes and serve as the source of intracellular AngII (Re and Cook, 2011).

ACE-independent, alternative pathways generating angiotensin peptides AngI, AngII, AngIII, Ang(1-7), and AngIV (Fig. 2) have been described. These are intricate, highly efficient endogenous pathways in cardiac and smooth muscle cells, renal tubular, mesenchymal, as well as inflammatory cells infiltrating various tissues. Contribution of alternative pathways involving chymases, tonins, neutral endopeptidases, and aminopeptidases can significantly vary relative to classic RAS in a pathologic setting (Carey and Siragy, 2003; Chua et al., 2010; Wasse et al., 2012; Naffah-Mazzacoratti et al., 2014; $\mathrm{Su}, 2014)$.

\section{Newer Components and Functional Axis of Renin- Angiotensin System}

The function of classic RAS was thought to be rather simple, to regulate formation of AngII as shown in Fig. 2. However, clinical application of RAS blockers demonstrated that these agents do not uniformly control circulating and tissue AngII levels. The concentration of AngII may often increase above pretreatment levels under therapy, which suggests that the beneficial effects are not due to blocking AngII production. Perhaps AngII is further metabolized to produce the beneficial effects. Paradigm shift discovery of angiotensin(1-7) [Ang(1-7)] as the antagonist of physiologic actions mediated by AngII and the discovery of angiotensin-converting enzyme 2 (ACE2) that produces Ang(1-7) in vivo has provided basis for this. More than a decade of research has unraveled a second arm of RAS (Fig. 2) that is composed of the monocarboxypeptidase ACE2, producing Ang(1-7) through hydrolysis of AngI or AngII, and counterbalancing the pressor arm constituted by renin-angiotensinogenAngI-ACE, leading to production of AngII (Ahmad et al., 2011). This arm of RAS is considered the counterregulatory axis. Both animal and clinical studies have emerged to define a role for ACE2 in regulating the progression of cardiovascular disease and pulmonary arterial hypertension. The activation of pulmonary ACE2 could serve as a novel therapeutic target in vivo (Bradford et al., 2010; Jiang et al., 2014). The ACE2/Ang(1-7) axis seems to be involved in many physiologic and pathophysiological processes in several systems and organs, especially by opposing the detrimental effects of inappropriate overactivation of the ACE/AngII axis (Passos-Silva et al., 2013). 
Blockade of RAS with drugs targeting various components disturbs feedback control of AngII levels by highly regulated release of renin. As a result, the increase in plasma renin causes AngII and aldosterone levels to surge. In addition, the levels of prorenin also increase, a key observation that led to the discovery of the prorenin receptor (Fig. 2) (Nguyen et al., 2002; Batenburg et al., 2004). Higher levels of prorenin than renin are seen in blood plasma during hypertension and diabetes. The prorenin receptor (PRR) is a ubiquitously expressed 350-amino acid protein, previously described as $\mathrm{Na} / \mathrm{H}^{+}$ATPase. PRR can bind both renin and prorenin and induce nonproteolytic prorenin activation and generation of AngI. PRR-mediated activation is a distinct mechanism from classic proteolytic activation of prorenin by an unidentified enzyme restricted to kidney. Renin inhibitors do not block the PRR activation of prorenin. PRR expression levels are high in brain regions, which could be particularly relevant because the expression of classic RAS components is low. The PRR-mediated oxidative stress in central nervous system is caused by increased reactive oxygen produced by overexpressed NOX2 and NOX4. This regulation is independent of AngII formation and involves ERKPI3K/Akt signals directly induced by PRR binding of prorenin (Peng et al., 2013). Neuron-specific PRR gene knockout prevents development of deoxycorticosterone acetate salt-induced hypertension and brain AngII production ( $\mathrm{Li}$ et al., 2014). PRR may thus be a critical membrane-bound prorenin receptor with signaling ability that regulates physiology. The PRR thus may contribute to angiotensin surges as well as directly activate transmembrane signaling independently. The discovery of PRR has renewed interest in the physiology of the RAS (Guang et al., 2012).

\section{E. Angiotensin Receptors Interpret Functions of Renin- Angiotensin System}

The concept of a plasma membrane receptor in target cells emerged based on specific recognition of AngII and stimulation of response such as adrenal steroidogenesis, nerve catecholamine release, and aortic contraction (Peach, 1977; Devynck et al., 1978). In the 1980s, pharmacological nonpeptide antagonists Dup753 and PD123177 were instrumental in demonstrating two types of AngII receptors in tissues, which were indistinguishable by peptide analogs (Chiu et al., 1989; Whitebread et al., 1989; Speth and Kim, 1990). Other angiotensin-derived metabolites such as Ang1-7, or Ang3-8 (AngIV) have all been shown to have distinct biologic activities (Peach, 1977; Ferrario et al., 1991; Wright et al., 1995; Iyer et al., 1998). Metabolism of AngII via aminopeptidases $\mathrm{A}$ and $\mathrm{N}$ generates AngIV (Padia and Carey, 2013). Effector organs responded differently to AngII and its metabolites based on the presence of distinctly different receptors, their abundance in target tissue, their selectivity for the agonists, their structure-activity relationships, signal transduction specificity, and regulation, desensitization and trafficking. G-protein-coupled receptors (GPCR) that elicit a response to AngII and Ang(2-8) (AngIII) are the angiotensin II type 1 ( $\mathrm{AT}_{1}$ receptor) and angiotensin II type $2\left(\mathrm{AT}_{2}\right.$ receptor $)$, which are extensively studied (Teerlink, 1996; de Gasparo et al., 2000). MAS, an orphan GPCR, has been functionally and pharmacologically linked to $\mathrm{Ang}(1-7)$ and is a strong candidate target for mediating the ACE2-Ang(1-7) axis of RAS. A class I membrane protease, insulin regulated amino peptidase (IRAP), is a strong candidate for AngIV receptor with distinctly different function compared with the GPCRs of RAS.

The decapeptide angiotensin I (AngI) serves as the reference for residue numbering of all angiotensin metabolites, as adopted by the International Society for Hypertension, The American Heart Association, and the World Health Organization (Dzau and Gibbons, 1987). Along this convention, the receptors for specific angiotensin metabolites are named Ang receptors (currently MAS is an exception). The classification of angiotensin receptors proposed in 1991 was updated in 2000 and 2014 (Bumpus et al., 1991; de Gasparo et al., 2000; Karnik et al., 2014).

The criteria used for inclusion of Ang receptors include the affinity and selectivity of agonists and antagonists for the receptor followed by transduction criteria, which is dependent on agonist-specific receptor-effector coupling. Finally, the gene and receptor sequence and the three-dimensional structure, if available, are the structural criteria. Signal coupling mechanisms and physiologic tissue responses in some instances have been major influencing factors on differentiating receptor types. Gene and receptor structures, cloning and heterologous expression combined with pharmacological validation were used in defining different receptors, which respond to specific metabolite fragment angiotensins, ultimately validating the structural basis for classification. Thus, a combination of several criteria was used in defining true receptor types. However, all criteria not met with equitable rigor for different Ang receptors remain a limitation.

Principles used for angiotensin receptor nomenclature were documented previously (de Gasparo et al., 2000). The receptors are $\mathrm{AT}_{1}-\mathrm{AT}_{3}$. Additional subdivisions (e.g., $\mathrm{AT}_{1 \mathrm{~b}}$ ) and species $\left(\mathrm{hAT}_{1}\right.$ or $\left.\mathrm{mAT}_{2}\right)$ are identified for pharmacologically defined receptor subtypes in different species. The Ang receptors $\mathrm{AT}_{1}$ and $\mathrm{AT}_{2}$ fully meet classification criteria, with IUPHAR Receptor Code of 2.1.Ang.01.000.00.00 and 2.1 Ang.02.000.00.00 (Humphrey and Barnard, 1998). They are seven transmembrane domains rhodopsin subclass GPCRs. The human genome contains single genes AGTR1 and AGTR2, which encode $\mathrm{AT}_{1}$ and $\mathrm{AT}_{2}$ receptors, respectively. The name $\mathrm{AT}_{3}$ receptor was originally assigned based on AngII binding described in the 
Neuro-2a mouse neuroblastoma cell line. AngII binding to sites in these cells was not blocked by the $\mathrm{AT}_{1}$-specific losartan, and the $\mathrm{AT}_{2}$-specific PD123319 and was not affected by GTP analogs (Chaki and Inagami, 1992b). Without the knowledge of the complete human genome at that time, cloning efforts directed at finding additional angiotensin receptor genes in humans lead to the realization of segmental duplications and rearrangements in unrelated loci but not a second $\mathrm{AT}_{1}$ receptor gene (Iafrate et al., 2004). Consequently, an AGTR3 gene is also not assigned in the completed human genome despite molecular identification of a putative cDNA clone for the proposed $\mathrm{AT}_{3}$ receptor. The existence of a genuine $\mathrm{AT}_{3}$ receptor is not confirmed at this time.

Additional angiotensin receptors (AngIV binding site and MAS) are proposed based on ligand interaction and physiologic functional criteria. The AngIV binding site may play a significant role in the central nervous system, kidney, and vasculature. The cloned AngIV binding site is an allosteric transmembrane zinc protease. The selectivity of endogenous AngIV for this receptor is not clear, and the physiologic characteristics evaluated in gene knockout and overexpression mouse models have not yielded a conclusive unifying picture. The signal transduction mechanisms of the AngIV binding site are unknown. Also, other functional surrogate AngIV binding sites proposed have generated some confusion. This transmembrane protease is thus a strong candidate for consideration as AngIV binding site. MAS is an exception to the Ang receptor nomenclature system, because it is an orphan GPCR capable of signal transduction in response to several unrelated peptides and angiotensin metabolites. The confusing pharmacology documented for this receptor, including the possibility of multiple endogenous ligands and confusing transduction modalities, is the reason for not elevating it to Ang(1-7) receptor. Multiple endogenous ligands are not unusual but AngIV binding site and MAS as physiologic entities do not rise to the level of acceptance as specific Ang receptors, because pharmacology and signaling are not rigorously established.

\section{The Angiotensin II Type 1 Receptor}

The lion's share of literature on RAS receptors belongs to the $\mathrm{AT}_{1}$ receptor as reflected by 4418 peer reviewed articles mined in our search (Fig. 1; Unal et al., 2014, http://www.guidetopharmacology.org/GRAC/ ObjectDisplayForward?objectId=34). Major effects on renal and cardiovascular physiology attributed to AngII are mediated through the $\mathrm{AT}_{1}$ receptor (Dinh et al., 2001). Chronic activation of the $\mathrm{AT}_{1}$ receptor can lead to disease states including hypertension, cardiac arrhythmia, stroke, diabetic nephropathy, and metabolic disorders (Audoly et al., 2000; de Gasparo et al., 2000; Zaman et al., 2002; Thomas and Mendelsohn,
2003), which are effectively treated using $\mathrm{AT}_{1}$ receptor blockers (Zaman et al., 2002; Billet et al., 2008; Akazawa et al., 2013; Michel et al., 2013; Seva Pessoa et al., 2013). The cDNAs for the $\mathrm{AT}_{1}$ receptor were cloned from rat smooth muscle and bovine adrenal gland (Murphy et al., 1991; Sasaki et al., 1991). The $\mathrm{AT}_{1}$ receptor of human, mouse, rabbit, pig, dog, turkey, and frog were characterized later. Human genome harbors a single gene, AGTR1, which mapped to chromosome 3q21-3q25. Rat and mouse genome harbors two distinct $\mathrm{AT}_{1}$ receptor genes, Agtr1a (chromosomes 17 and 13, respectively) and Agtr $1 b$ (chromosomes 2 and 3, respectively). The $\mathrm{AT}_{1 \mathrm{a}}$ receptor and $\mathrm{AT}_{1 \mathrm{~b}}$ receptor share 95\% amino acid sequence homology. Noncoding regions of their genes are strikingly different, suggesting possible differences in tissue-specific expression and regulation of $\mathrm{AT}_{1 \mathrm{a}}$ receptor and $\mathrm{AT}_{1 \mathrm{~b}}$ receptor (de Gasparo et al., 2000). Indeed, both receptor subtypes are pharmacologically and functionally identical, but they differ in tissue distribution and transcriptional regulation. The $\mathrm{AT}_{1 \mathrm{a}}$ receptor is well expressed in most cardiovascular tissues and is the principal regulator of blood pressure. The $\mathrm{AT}_{1 \mathrm{~b}}$ receptor expression is limited to endocrine tissues such as the adrenal and pituitary glands (Kakar et al., 1992; Ito et al., 1995; Sugaya et al., 1995; Chen et al., 1997). The AGTR1 gene contains five exons and four introns, out of which exon 5 codes for the hAT 1 receptor polypeptide (Sasaki et al., 1991). Human AGTR1 splice variant mRNAs containing exon 2 are poorly translated. In contrast, mRNA splice variants, which harbor exon 3 , produce a longer $\mathrm{hAT}_{1}$ receptor isoform with additional $32 \mathrm{~N}$-terminal amino acids. The long-form of $\mathrm{hAT}_{1}$ receptor displayed a threefold diminished affinity for AngII. Splice variants of $\mathrm{hAT}_{1}$ receptor may ultimately affect AngII responsiveness in a given tissue (Warnecke et al., 1999; Elton and Martin, 2003). The early characterization of $\mathrm{AT}_{1}$ receptor pharmacology and signal transduction property is comprehensively reviewed by de Gasparo et al. (2000).

\section{A. Structure-Function}

The genomic DNA of all mammalian species contains an open reading frame for 359 amino acid residues, yielding $\sim 41 \mathrm{kDa}$ calculated molecular weight for $\mathrm{AT}_{1}$ receptor (Guo et al., 2001). $\mathrm{AT}_{1}$ receptor belongs to the rhodopsin branch of the GPCR superfamily. The extracellular domain consists of the $\mathrm{N}$ terminus and the extracellular loops (ECLs) and contains three $N$-glycosylation sites. Four cysteine residues in the extracellular domain of the $\mathrm{AT}_{1}$ receptor are predicted to form two disulfide bonds (Fig. 3A), which are prone to inactivation by dithiothreitol and other reducing agents (Warnecke et al., 1999). The three intracellular loops form the $\mathrm{G}$ protein activation domain. The cytoplasmic C-terminal tail contains phosphorylation sites for serine/threonine kinases, including protein kinase $\mathrm{C}$ (PKC) and GPCR kinases. Functionally, the $A T_{1}$ receptor is primarily coupled through the $G_{q}$ protein to 
phospholipases C, A2, and D. Similar to other prototypical GPCRs, agonist activation leads to desensitization and internalization of $\mathrm{AT}_{1}$ receptor.

The three-dimensional structure at room temperature with 2.9 -A resolution was determined for $\mathrm{hAT}_{1}$ receptor bound to the experimental antihypertensive agent ZD7155 using a novel, X-ray-free approach through collaborative efforts (Zhang et al., 2015). The threedimensional structure confirms a canonical seventransmembrane (TM) $\alpha$-helical architecture with precise boundaries for $\mathrm{N}$ terminus, three extracellular loops (ECL1-3), three intracellular loops (ICL1-3), an amphipathic helix VIII, and the C terminus (Figs. 2 and 3). The $\mathrm{AT}_{1}$ receptor three-dimensional structure is most similar to chemokine and opioid receptors. The predicted disulfide bonds, Cys18-Cys274 connecting the $\mathrm{N}$ terminus and ECL3, and Cys101-Cys180 connecting helix III and ECL2 are confirmed. The ECL2 of AT 1 receptor exhibits a $\beta$-hairpin secondary structure as in other peptide GPCRs (Fig. 3B). Intriguingly, ECL2 of $\mathrm{AT}_{1}$ receptor serves as an epitope for the agonistic autoantibodies in preeclampsia and malignant hypertension (Unal et al., 2012; Xia and Kellems, 2013). The conserved DRY motif in helix III and the NPxxY motif in helix VII of $\mathrm{AT}_{1}$ receptor were proposed to participate in receptor activation (Oliveira et al., 2007).

An agonist bound $\mathrm{AT}_{1}$ receptor structure is unavailable at this time. However extensive site-directed mutagenesis studies combined with biochemical and pharmacological experiments on mammalian $\mathrm{AT}_{1}$ receptor have provided insights into AngII binding, mechanism of receptor activation, G-protein interaction, as well as regulation by desensitization and internalization (Hjorth et al., 1994; Marie et al., 1994; Schambye et al., 1994; Noda et al., 1995a; Yamano et al., 1995; Hunyady et al., 1996, 1998; Karnik et al., 1996; Monnot et al., 1996; Balmforth et al., 1997; Groblewski et al., 1997; Inoue et al., 1997; Han et al., 1998).

The current view is that AngII binding to the $\mathrm{AT}_{1}$ receptor involves two salt bridges, between the $\alpha$-carboxyl group of AngII and $\mathrm{Lys}^{199}$ of $\mathrm{AT}_{1}$ receptor and between the guanidinium group of Arg-2 in AngII and Asp ${ }^{281}$ in the receptor (Yamano et al., 1992; Feng et al., 1995; Noda et al., 1995a; Miura et al., 2003a). Ligand-crosslinking approach showed interactions between Phe- 8 of AngII and $\mathrm{Phe}^{293}$ and $\mathrm{Asn}^{294}$ in $\mathrm{AT}_{1}$ receptor in $\mathrm{TM}$ domain (Perodin et al., 2002). Another interaction observed by crosslinking between Val-3 of AngII and $\mathrm{Ile}^{172}$ in $\mathrm{AT}_{1}$ receptor is consistent with an ion pair suggested between Asp-1 in AngII and His ${ }^{183}$ by mutagenesis data (Feng et al., 1995; Boucard et al., 2000). $\mathrm{Arg}^{23}$ in $\mathrm{N}$ terminus of $\mathrm{AT}_{1}$ receptor may be essential for binding AngII (Santos et al., 2004a). Thus, the hydrophobic carboxyl terminal region of AngII appears to enter the TM-domain core of the receptor and amino terminal region of AngII appears to interact with extracellular region of the $\mathrm{AT}_{1}$ receptor. A two-step model for binding of AngII to $\mathrm{AT}_{1}$ receptor has been proposed (Le et al., 2002; Feng et al., 2005). Boucard et al. (2000) suggested an extended conformation of $\mathrm{AT}_{1}$ receptorbound AngII (Perodin et al., 2002; Fillion et al., 2013). The methionine proximity mapping approach they used identified details of the residues lining the AngII binding
A

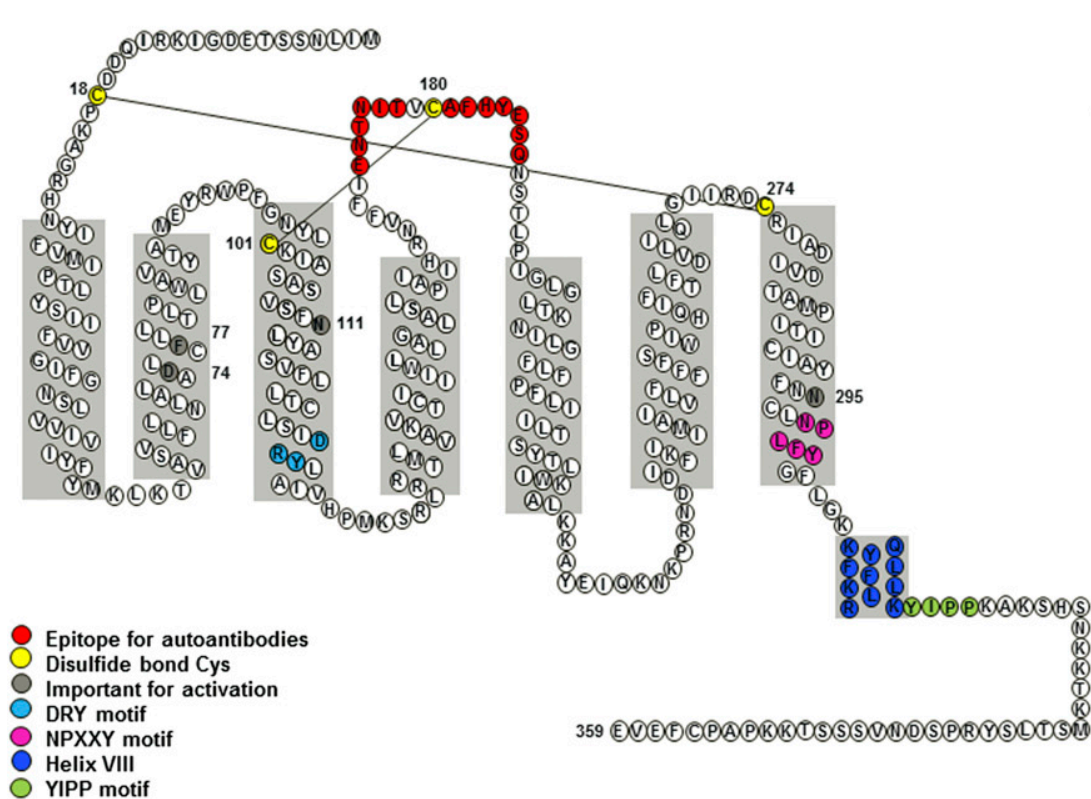

B

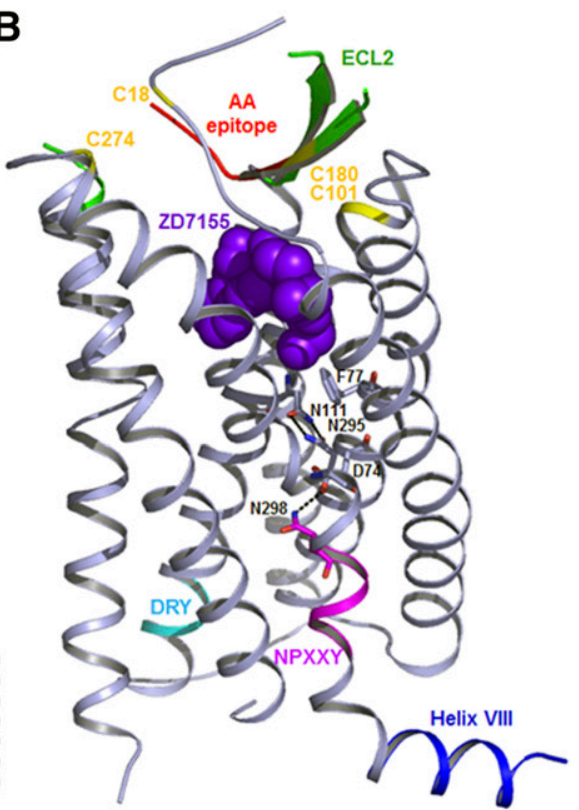

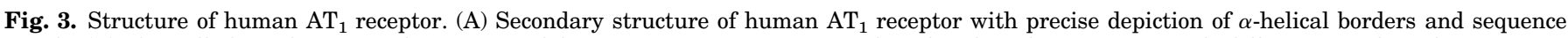

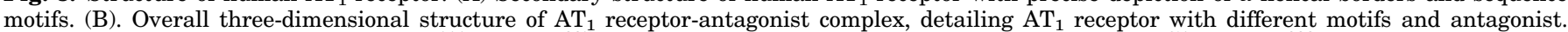

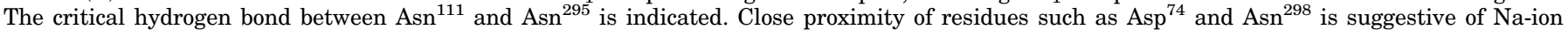
coordination site. Sodium concentration may modulate receptor activation by agonists. 
pocket of $\mathrm{AT}_{1}$ receptor. These residues include $\mathrm{Phe}^{77}$, $\mathrm{Leu}^{112}, \mathrm{Tyr}^{113}, \mathrm{Phe}^{249}$, Trp ${ }^{253}, \mathrm{His}^{256}, \mathrm{Thr}^{260}$, and $\mathrm{Phe}^{293}$, $\mathrm{Asn}^{294}, \mathrm{Asn}^{295}$, Cys ${ }^{296}$, and Leu ${ }^{297}$ (Correa et al., 2002; Clement et al., 2005, 2006, 2009). Almost all of these residues are within $3-\AA$ distance of the bound antagonist in the crystal structure, which substantiates the idea that the agonist, AngII, and $\mathrm{AT}_{1}$ receptor antagonists share an overlapping binding pocket of the $\mathrm{AT}_{1}$ receptor.

Intrinsic stability prevents spontaneous activation of $\mathrm{AT}_{1}$ receptor, and agonists overcome this stability barrier during activation. AngII is proposed to mediate activation of $\mathrm{AT}_{1}$ receptor through stacking interactions between Phe- 8 of AngII and His ${ }^{256}$ (Noda et al., 1995a) and between Tyr-4 of AngII and $\mathrm{Asn}^{111}$ in $\mathrm{AT}_{1}$ receptor (Noda et al., 1996; Miura et al., 1999). Interhelical interaction between $\mathrm{Asn}^{111}$ and $\mathrm{Tyr}^{292}$ in the inactive receptor is proposed to be disrupted upon AngII binding, allowing Tyr ${ }^{292}$ to interact with $\mathrm{Asp}^{74}$ (Joseph et al., 1995). Smaller residue substitutions for $\mathrm{Asn}^{111}$ have been shown to induce constitutively activated $\mathrm{AT}_{1}$ receptor conformation (Groblewski et al., 1997; Feng et al., 1998; Miura et al., 1999). The three-dimensional structure suggests that Asn ${ }^{111}$ hydrogen bonds with $\mathrm{Asn}^{295}$, and disruption of this interaction may cause constitutive activation of $\mathrm{AT}_{1}$ receptor. Several other TM mutations that potentially disrupt intrinsic stability in $\mathrm{AT}_{1}$ receptor are reported to produce constitutive activation, including $\mathrm{Asn}^{295}$, $\mathrm{Asp}^{125}, \mathrm{Phe}^{77}$, $\mathrm{Leu}^{112}$, $\mathrm{Leu}^{118}$, Leu ${ }^{195}, \mathrm{Ile}^{245}$, and Leu ${ }^{305}$ (Parnot et al., 2000). Nikiforovich et al. (2005) showed that $\mathrm{AT}_{1}$ receptor constitutive activity is also elicited when bulkier residues were introduced in TMIII without mutating Asn ${ }^{111}$. They proposed that by mutating Asn ${ }^{111}$ and adjacent bulky residues, a cascade of conformational perturbations activated the receptor.

Activation of $\mathrm{AT}_{1}$ receptor is shown to be associated with translation and rotation of TM helices, including TMII, TMIII, TMV, TMVI, and TMVII by two independent methods, reporter cysteine accessibility mapping and methionine proximity analysis (Miura and Karnik, 2002; Boucard et al., 2003; Miura et al., 2003b; Martin et al., 2004, 2007; Domazet et al., 2009a,b; Arsenault et al., 2010a). The residues Met ${ }^{30}$ and $\mathrm{Thr}^{33}$ in TMI, as well as $\mathrm{Arg}^{167}$ and $\mathrm{Val}^{169}$ in ECL2, are shown to interact with AngII, but these regions undergo very little movement during $\mathrm{AT}_{1}$ receptor activation (Yan et al., 2010). Only subtle structural changes were identified between the $\mathrm{AT}_{1}$ receptor and its constitutively active form (Clement et al., 2006). Binding of structurally different ligands could produce different active receptor conformations (Miura et al., 2012).

ECL2 is a critical determinant of ligand-specific conformational changes resulting in activation or inhibition of the $\mathrm{AT}_{1}$ receptor (Unal et al., 2010). Unal et al. (2010) suggested that ECL2 might assume a lid conformation induced upon binding both agonist and antagonist but exposing distinct residues around the highly conserved disulfide bond between $\mathrm{Cys}^{180}$ and $\mathrm{Cys}^{101}$. A role in coupling the movements of TM helices to the ECL2 during receptor activation was suggested (Ohyama et al., 1995; Karnik et al., 2003; Unal et al., 2010). Interestingly, the ECL2 assumed a lid conformation in the gain-of-function mutant $\mathrm{N} 111 \mathrm{G}-\mathrm{AT}_{1}$ receptor without agonist stimulation (Unal et al., 2013). In contrast, AngII did not induce a lid conformation in the loss-offunction mutant D281A, which is consistent with poor AngII binding in this mutant. However, a lid conformation was regained when an AngII analog that is specific for D281A mutant bound the mutant receptor. On the basis of these results an emerging paradigm of domain coupling facilitated by long-range interactions was proposed. Domain coupling has been postulated as a mechanism for explaining a range of GPCR conformations with different functional consequences regulated not only by classic agonists and antagonists, but also by a variety of nonclassic factors including receptor-interacting proteins, dimerization, and activation by autoantibody (Unal and Karnik, 2012).

Mutational analysis has shown that $\mathrm{Tyr}^{127}$ to $\mathrm{Met}^{134}$ region (ICL2) and the $\mathrm{Ile}^{238}$ to $\mathrm{Phe}^{239}$ region (ICL3) are important for $\mathrm{G}_{\mathrm{q} / 11}$ activation by the $\mathrm{AT}_{1}$ receptor (Miura et al., 2000; Zhang et al., 2000; Gaborik et al., 2003). Critical single residues in the intracellular region of the $\mathrm{AT}_{1}$ receptor required for G-protein activation include $\mathrm{Arg}^{126}$ (TMIII), Tyr ${ }^{215}$ (TM V), Leu ${ }^{222}$ (ICL 3), $\mathrm{Tyr}^{312}$, Phe $^{313}$, and Leu ${ }^{314}$ (TMVIII) (Oliveira et al., 2007). The carboxyl tail of $\mathrm{AT}_{1}$ receptor regulates $\mathrm{AT}_{1}$ receptor desensitization and internalization (Hunyady and Catt, 2006; Smith and Luttrell, 2006) and it also directly associates with downstream effectors and plays crucial roles in signal transduction, internalization, and other functions of the receptor (Cruse et al., 1992; Ali et al., 1997; Venema et al., 1998a; Horiuchi et al., 2012). A stretch of C-terminal residues 305-320 in $\mathrm{AT}_{1}$ receptor is known as the helix VIII (Huynh et al., 2009). Multiple lines of evidence suggest contribution of helix VIII to $\mathrm{AT}_{1}$ receptor expression and trafficking, G-protein coupling and activation, receptor internalization, dimerization, and signaling by mediating protein-protein and protein-lipid interactions. The Tyr-Ile-Pro-Pro (YIPP, amino acids 319-322 in Fig. 3A) motif within helix VIII is essential for activation of the Jak-STAT pathway, PLCgamma phosphorylation, and the AngII-induced intracellular calcium transients (Venema et al., 1998a).

\section{B. Pharmacology}

Nonpeptide $\mathrm{AT}_{1}$ receptor antagonists, referred to as $\mathrm{AT}_{1}$ receptor blocking (ARB) drugs, are a cornerstone of therapy for not only lowering blood pressure and protecting renal damage, but are under consideration for a variety of other human disease conditions. At least eight different ARBs are clinically available at this time. They are losartan, candesartan, olmesartan, telmisartan, eprosartan, irbesartan, valsartan, and 
A

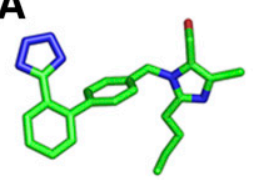

Losartan

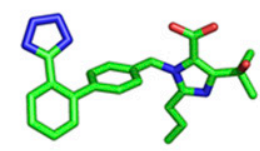

Olmesartan

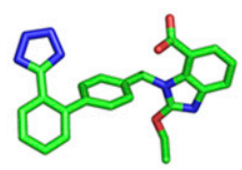

Candesartan
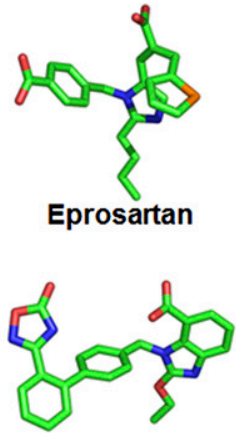

Azilsartan

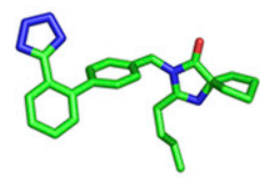

Irbesartan

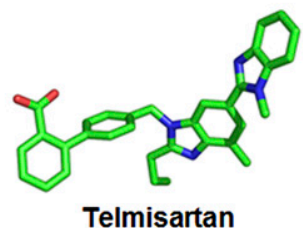

Telmisartan

B

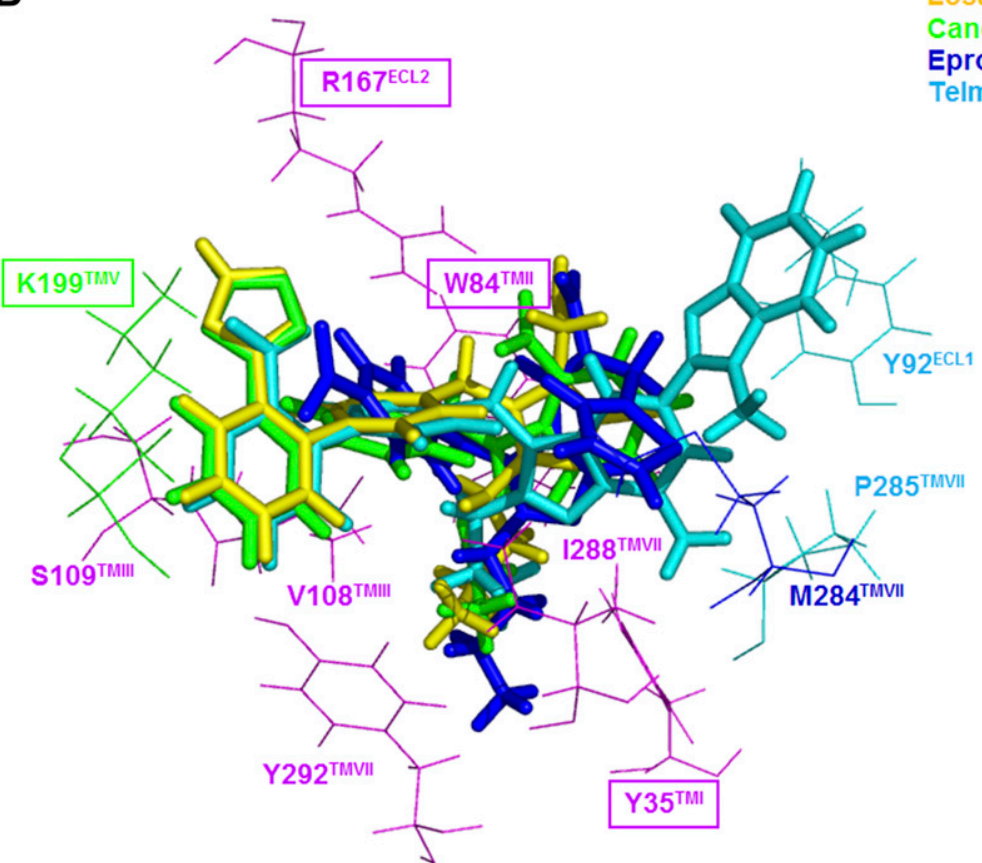

Fig. 4. (A) Three-dimensional structural details of $A R B s$ in clinical use; and (B) interaction with human $\mathrm{AT}_{1}$ receptor. Differences in the efficacy of different ARBs may be due to small differences in interaction of shown ARBs with residues such as Tyr ${ }^{92}$, Trp ${ }^{182}, \operatorname{Lys}^{199}$, Met ${ }^{284}$, and $\mathrm{P}^{285}$.

azilsartan (Fig. 4A). ARB development efforts critically focus on binding to $\mathrm{AT}_{1}$ receptor and oral bioavailability, insurmountability, inverse agonism (Kohara et al., 1996; Miura et al., 2006; Fujino et al., 2010). Candesartan cilexitil, losartan, and olmesartan medoxomil are prodrugs of candesartan, EXP3174, and olmesartan, respectively (Schmidt and Schieffer, 2003). Most ARBs do not cross the blood-brain barrier but central effects may be observed due to prolonged treatment or under pathologic conditions where the blood-brain barrier may become more permissive. Telmisartan is reported to cross the blood-brain barrier, but losartan, candesartan, olmesartan, azilsartan, and valsartan are reported to poorly cross the blood-brain barrier. Effects on the central nervous system are reported in preclinical studies for several ARBs, which may afford beneficial effects in Alzheimer's disease in addition to blood pressure lowering (Duron and Hanon, 2010). Physiologic responses to in vivo antagonism of the $\mathrm{AT}_{1}$ receptor have been quantified by measurement of renin release (Munafo et al., 1992; Muller et al., 1994; Maillard et al., 2002), aldosterone release (Ogihara et al., 1995), and plasma NO production (Gossmann et al., 2001) in response to AngII in humans.

1. Pharmacophore Structure-Activity Relationship. Structurally, the biphenyl tetrazole seen in losartan, EXP3174, ZD7155, and candesartan or an acidic moiety as seen in telmisartan, 5-oxo-1,2,4-oxadiazole, as in azilsartan change lipophilicity of ARBs (Vyas and Ghate, 2010). Eprosartan has a different structure, biphenyltetrazole replaced with benzoic acid. Consequently, ARBs are designed to exploit hydrophobic interaction of the phenyl rings with transmembrane core of $\mathrm{AT}_{1}$ receptor and bind basic residues in $\mathrm{AT}_{1}$ receptor forming ionic interactions with the acidic moieties. Losartan has an imidazole with $\mathrm{Cl}$ at the other end of the molecule, 
and its metabolite, EXP3174, has a COOH substituent. Sartans present a greater variety of structures at this end that probably account for some of their functional differences. Olmesartan and losartan are closely related. Irbesartan has a cyclopentyl ring incorporated in place of the $\mathrm{Cl}$. A benzimidazole is substituted in candesartan and azilsartan; two benzimidazoles are attached in telmisartan to produce an unusual structure for this $\mathrm{ARB}$. The unique structure of valsartan lacks a nitrogen containing heterocycle. The imidazole ring in eprosartan has a large alkyl chain substituent. The difference in structure is expected to cause the ARBs to bind in slightly different ways.

In the crystal structure of $\mathrm{AT}_{1}$ receptor bound to ZD7155, the ligand-binding pocket shows exactly how ARBs might interact with $\mathrm{AT}_{1}$ receptor (Fig. 3). The $\mathrm{AT}_{1}$ receptor residues, mainly from helices I, II, III, VII, and ECL2, directly interact with the ligand. Side chains of $\mathrm{Arg}^{167(\mathrm{ECL} 2)}$ and $\mathrm{Tyr}^{35(\mathrm{TM} 1)}$ form ionic and polar interactions with ZD7155. The acidic tetrazole moiety closely interacts with the basic $\mathrm{Arg}^{167(\mathrm{ECL} 2)}$, and this residue may be primarily responsible for ARB-binding affinity and selectivity for $\mathrm{AT}_{1}$ receptor. $\mathrm{Tyr}^{35(\mathrm{TM} 1)}$ and the naphthyridin-2-one moiety of ZD7155 form hydrogen bonds. Trp ${ }^{84(\mathrm{TM} 2)}$ of $\mathrm{AT}_{1}$ receptor forms $\pi-\pi$ interaction with the naphthyridin-2-one moiety of ZD7155. Additionally, residues $\mathrm{Ile}^{31 \text { (TM1) }}, \mathrm{Val}^{108(\mathrm{TM} 3)}$, Leu ${ }^{112(\mathrm{TM} 3)}$, and $\mathrm{Tyr}^{292(\mathrm{TM} 7)}$ in $\mathrm{AT}_{1}$ receptor ligand-binding pocket interact hydrophobically with ZD7155. Most of the other contacts for $\mathrm{ZD} 7155$ binding to $\mathrm{AT}_{1}$ receptor, however, are mediated by residues, including $\mathrm{Tyr}^{87(\mathrm{TM} 2)}$, Thr ${ }^{88(T M 2)}$, Ser $^{105(T M 3)}$, Ser $^{109(T M 3)}$, Ala $^{163(T M 4)}$, Phe $^{182(E C L 2)}$, Pro ${ }^{285(T M 7)}$, and Ile ${ }^{288(T M 7)}$ (Zhang et al., 2015).

Shape of the binding cavity is determined by the secondary structures and disulfide crosslinking patterns of the extracellular loop region, proline and nonproline kinks in 7TM helical bundle, and other local variations, resulting in deviations in the extracellular tips of TM helices. Specific structural variations create a remarkable variety of sizes, shapes, and electrostatic properties of the ligand-binding pockets in different GPCRs. The ZD7155 binding site in $\mathrm{AT}_{1}$ receptor partially overlaps with known ligand binding sites in the chemokine and opioid receptors in which the cavity is larger, more open, and located closer to the extracellular surface, a general feature of GPCRs that recognize diffusible small-molecule ligands. Some of the residues that comprise the ligand-binding pockets are conserved among these structurally similar peptide GPCRs, including Tyr ${ }^{\text {(TM1) }}$ and Trp ${ }^{\text {(TM2) }}$, and the majority of the residues forming the ligand binding of these receptors are close to the extracellular boundaries of the helices. $\mathrm{Arg}^{167(\mathrm{ECL} 2)}$ is one of the three critical residues that holds the antagonist close to the extracellular side and it is a unique residue of $\mathrm{AT}_{1}$ receptor compared with other structurally similar peptide GPCRs.
Docking simulations of the clinically used antihypertensive ARBs show that they bind in similar orientations and interact with the three critical residues, $\mathrm{Arg}^{167(\mathrm{ECL} 2)}$, Trp ${ }^{84(\mathrm{TM} 2)}$, and $\mathrm{Tyr}^{35(\mathrm{TM} 1)}$ in the $\mathrm{AT}_{1}$ receptor ligand-binding pocket (Fig. 3). Details of the interactions with residues $\mathrm{Phe}^{77(\mathrm{TMII})}$, $\mathrm{Tyr}^{87(\mathrm{TMII})}$, Ser $^{105 \text { (TMIII) }}, \mathrm{Val}^{108 \text { (TMIII) }}$, Ser ${ }^{109 \text { (TMIII) }}$, Leu ${ }^{112(\mathrm{TMIII})}$, $\mathrm{Ala}^{163(\mathrm{TMIV})}, \mathrm{Phe}^{182(\mathrm{ECL} 2)}, \mathrm{Ile}^{288(\mathrm{TMVII})}$, and $\mathrm{Tyr}^{292(\mathrm{TMVII})}$, which shape the ligand-binding pocket of the $\mathrm{AT}^{1}$ receptor, are different for different ARBs. For example, one of the common features among these ARBs is a short alkyl tail with 2-4 carbons extending into a narrow hydrophobic pocket formed by Tyr ${ }^{35 \text { (TM1) }} \mathrm{Phe}^{\text {77(TM2) }}$, $\mathrm{Val}^{108(\mathrm{TM} 3)}, \mathrm{Ile}^{288(\mathrm{TM} 7)}$, and $\mathrm{Tyr}^{292(\mathrm{TM} 7)}$. Losartan is a surmountable antagonist with lower binding affinity to $\mathrm{AT}_{1}$ receptor compared with the later developed ARBs (Takezako et al., 2004; Miura et al., 2011). Docking results suggest that $\mathrm{Arg}^{167(\mathrm{ECL} 2)}$ forms a salt bridge with only the tetrazole moiety of losartan but lacks other polar interaction and its interaction with $\mathrm{Tyr}^{35(\mathrm{TM} 1)}$; distances and angles for hydrogen bonding are suboptimal. The lower binding affinity and surmountable property of losartan for $\mathrm{AT}_{1}$ receptor is likely due to this. In contrast, candesartan docking results indicate that it forms optimal interaction with the pocket. Lys ${ }^{199(T M 5)}$ may form an additional salt bridge with the tetrazole moiety and further stabilize candesartan binding. Crystal structure and docking analyses suggest that Lys ${ }^{199(T M 5)}$ provides conformational heterogeneity in the $\mathrm{AT}_{1}$ receptor; the amino group of this residue may reach the acidic moieties of ARBs by forming salt bridges (as in the cases of candesartan and telmisartan) or through water-mediated interactions with other ARBs (Zhang et al., 2015). The structure of nonpeptide antagonists mimics the contact points of AngII side chains, and their binding sites overlap with the AngII binding pocket in $\mathrm{AT}_{1}$ receptor as also supported by mutagenesis and crosslinking experiments (Yamano et al., 1992; Ji et al., 1994; Noda et al., 1995b; Vanderheyden et al., 2000a; Takezako et al., 2004).

Current literature is dominated by studies on losartan, candesartan, valsartan, irbesartan, olmesartan, and azilsartan that share a common biphenyl-tetrazole scaffold. Two ARBs in clinical practice differ, however. Telmisartan (also known as BIBR 277) structure is unusual, with two benzimidazole moeities attached (Fig. 4A). Eprosartan (also known as SK\&F 108566), is the other ARB with the most differentiated structure. Telmisartan and eprosartan treatment benefits are not fully characterized, but potential benefits due to differences in their structural features are possible. These ARBs interact Arg $167^{\mathrm{ECL} 2}$, Tyr35 ${ }^{\mathrm{TM} 1}$, and $\operatorname{Trp} 84^{\mathrm{TM} 2}$ similar to other biphenyl-tetrazole ARBs, and mutants of these residues dramatically reduced their binding. However, the three-dimensional structure modeling studies predicted that telmisartan and 
eprosartan bind, interacting with significantly different residues (Fig. 4B). Eprosartan extends interaction into the hydrophobic subpocket consisting of Ile $288^{\mathrm{TM} 7}$ and Tyr292 ${ }^{\mathrm{TM} 7}$. Alanine substitution of both Ile $288^{\mathrm{TM} 7}$ and Tyr292 ${ }^{\mathrm{TM} 7}$ specifically decreased eprosartan-binding affinities. Two consecutive benzimidazole moieties in telmisartan were predicted to make additional $\pi-\pi$ contacts with Tyr92 ${ }^{\text {ECL1 }}$ (Fig. 4B). Mutation of Tyr92 ${ }^{\text {ECL1 }}$ to alanine, although mostly neutral for other ARBs tested, significantly lowered affinity for telmisartan. Furthermore, Ile288Ala mutation also has a discriminating effect on telmisartan binding. Structural analysis reveals a novel paradigm, molecular recognition through extended interaction with receptor subpockets. This may suggest possible difference in outcome from blockade of $\mathrm{AT}_{1}$ receptor function using different $\mathrm{ARBs}$ (Zhang et al., 2015).

2. Inverse Agonism. Classic competitive antagonism model does not easily explain pharmacological behavior of most ARBs, therefore, more recent models to describe drug properties have been adopted. It is now generally accepted that $\mathrm{AT}_{1}$ receptor has small but functionally significant constitutive activity (Unal and Karnik, 2014), and perhaps most ARBs do reduce constitutive activity of receptor in the absence of agonist, a phenomenon called inverse agonism. Inverse agonism of most ARBs is detected using constitutively active mutants of $\mathrm{AT}_{1}$ receptor created by site-directed mutagenesis (Unal and Karnik, 2014). Inverse agonism has been shown for EXP3174, olmesartan, telmisartan, valsartan, and azilsartan (Noda et al., 1996; Miura et al., 2003a, 2006, 2013; Feng et al., 2005; Bhuiyan et al., 2009; Ojima et al., 2011). Whether losartan possesses inverse agonist properties remains controversial, because it was noted in one report (Bhuiyan et al., 2009) but not in further studies (Miura et al., 2003b; Feng et al., 2005). Clinical relevance of inverse agonism of ARBs is still debated. Kiya et al. (2010) studied nephroprotective properties of olmesartan and its analog lacking inverse agonism in Dahl salt-sensitive rats. Olmesartan lowered urinary protein excretion by $\sim 25 \%$, but its close structural analog that lacked inverse agonism did not show nephroprotective effect. Differential effect of inverse agonist ARBs on proteinuria remains to be systematically studied.

3. Insurmountable and Reversible Antagonism. Most clinically used ARBs exhibit an atypical competitive and reversible interaction with $\mathrm{AT}_{1}$ receptor. The AngII concentration-response curves in the presence of ARBs shift toward higher concentrations, but with reduced maximal response. In experimental settings, a very high concentration of AngII cannot overcome the ARB inhibition fully. Most clinically used ARBs harbor this behavior (van Liefde and Vauquelin, 2009), but insurmountable antagonism of ARBs remains a rather descriptive term. Site-directed mutagenesis studies to identify receptor mechanisms have not provided a satisfactory answer (Vanderheyden et al., 2000b; Verheijen et al., 2003; Van Liefde and Vauquelin, 2009). Insurmountable antagonism of candesartan (Noda et al., 1993; Ojima et al., 1997), olmesartan, or EXP3174 was shown to increase with duration of preincubation with the receptor (Mizuno et al., 1995). Antagonism lasted for hours upon washout for many ARBs, including candesartan, EXP3174, olmesartan, telmisartan, and azilsartan. In insurmountable antagonism, the slow dissociation rates from the receptor may suggest conformational effects of ARB binding with clinically relevant functional consequences. The dissociation half-lives of the $\mathrm{hAT}_{1}$ receptor has been measured, and the order of dissociation was found to be telmisartan, olmesartan, candesartan, valsartan, and losartan, respectively, with $t_{1 / 2}$ of $213,166,133,70$, and 67 minutes (Vanderheyden et al., 2000b; Verheijen et al., 2000; Kakuta et al., 2005)

4. Biased Agonism. The ability of a given receptor to selectively activate a signaling path compared with another is defined as "biased agonism" or "ligand-directed" signaling. Biased ligands, also known as functionally selective agonists, can involve a subset of a receptor's normal signaling repertoire by stabilizing different receptor conformational states than are stabilized by endogenous "unbiased" ligands (Kenakin, 2007; Violin and Lefkowitz, 2007; DeWire and Violin, 2011; Godin and Ferguson, 2012). Functional selectivity exhibited by several AngII analogs has forced redefinition of receptor agonism paradigm to allow discrete receptor activation states from ligands with different efficacy for individual responses (Kenakin, 2005; Galandrin et al., 2007; Urban et al., 2007; Hansen et al., 2008; Lyngso et al., 2009). AngII peptide modification led to the description of agonists, partial agonists, and antagonists (Miura et al., 1999; Miura and Karnik, 1999; Oliveira et al., 2007). Hormone AngII is a full agonist with pluridimensional efficacy for all signals activated by $\mathrm{AT}_{1}$ receptor, whereas AngII analog such as $\left[\mathrm{Sar}^{1}, \mathrm{Il}^{4}, \mathrm{Ile}^{8}\right]$ AngII (SII-AngII) is a biased agonist that does not activate $\mathrm{G}$ protein signaling but allows receptor phosphorylation and subsequent $\beta$-arrestin-mediated signaling (Kenakin, 2003, 2005; Urban et al., 2007). This discovery has opened the possibility for novel designer ligands that may activate only some of $\mathrm{AT}_{1}$ receptor signals. This development holds great promise for the treatment of diseases such as $\mathrm{HF}$ and hypertension (Holloway et al., 2002; Wei et al., 2003; Daniels et al., 2005; Aplin et al., 2007a,b; Hansen et al., 2008; Shukla et al., 2008; Sauliere et al., 2012).

Insight into the pluridimensional efficacy of AngIIanalogs was initially described by Thomas et al. (2000) and Holloway et al. (2002). These authors studied the IP3 signaling, MAPK signaling, phosphorylation, and internalization of $\mathrm{AT}_{1}$ receptor in response to AngII and several AngII analogs. Internalization of $\mathrm{AT}_{1}$ receptor-EGFP was unaffected by substitution of Tyr-4 
and Phe-8 in AngII that abolish IP3 signaling (Holloway et al., 2002). On the other hand, substitution of Asp-1 with alanine and substitutions of $\mathrm{Phe}^{8}$ with alanine, isoleucine, diphenylalanine, and $\beta$-cyclohexylalanine significantly inhibited phosphorylation. The activation of MAPK was inhibited by Phe- 8 substitutions, which did not equally inhibit IP3 production or receptor phosphorylation, indicating overlapping but distinct AngII-AT $_{1}$ receptor interactions. Yee et al. (2006) described three distinct activated states of $\mathrm{AT}_{1}$ receptor based on the response of several $\mathrm{AT}_{1}$ receptor mutants to AngII and SII-AngII. Three active states proposed include AngII-mediated G-protein signaling, AngIImediated G-protein-independent ERK1/2 signaling, and II-AngII-mediated G-protein-independent ERK1/2 signaling (Yee et al., 2006), thus linking receptor ligand combinations to generation of observed functional selectivity. SII-AngII is extensively studied for engagement of GRKs, antiapoptotic signals, chemotaxis, cell growth, and proliferation (Hunton et al., 2005; Yee et al., 2006; Aplin et al., 2007a,b; DeWire et al., 2008). An analog such as TRV120027 is a 30 -fold more potent $\beta$-arrestin-biased $\mathrm{AT}_{1}$ receptor ligand that has been studied in vitro and in vivo in rats and dogs and is now in Phase II clinical studies for the treatment of acute HF. The preclinical data are reported to indicate that TRV120027 has the potential to support heart, vasculature, and kidney function in acute HF (Violin et al., 2010; Boerrigter et al., 2011). TRV120027 may block the effects of elevated AngII on the vasculature and kidney, while protecting or enhancing prosurvival and contractility signals in the heart.

As seen in the case of many GPCR antagonists, ARBs do not cause internalization of the $\mathrm{AT}_{1}$ receptor and block agonist-induced internalization. Unbiased antagonism by losartan, telmisartan, and valsartan are reported in internalization and desensitization (Violin et al., 2010). However, candesartan, losartan, telmisartan, and valsartan allow substantial internalization of constitutively active $\mathrm{AT}_{1}$ receptor mutants (Bhuiyan et al., 2010). This phenomenon may reflect hidden biased agonism by these ARBs, a phenomenon that needs to be rigorously evaluated. Instances of a compound acting as an antagonist for one response and an agonist for a different response mediated by the same receptor are known (Patel et al., 2012). These findings contrast with studies in which candesartan binding did not induce internalization of a GFP-AT $\mathrm{T}_{1}$ receptor in Chinese hamster ovary (CHO) cells (Le et al., 2005). However, these aspects are yet to be described for $\mathrm{AT}_{1}$ receptor and its peptide as well as nonpeptide ligands.

\section{Mouse Models}

No major abnormalities of the cardiac and vascular system are reported in mice lacking the $\mathrm{AT}_{1 \mathrm{a}}$ receptor $\left(\mathrm{AT}_{1 \mathrm{a}}\right.$ receptor-null). These mice develop normally but show a marked reduction of systolic blood pressure
(Yang et al., 2010). In contrast, the $\mathrm{AT}_{1 \mathrm{~b}}$ receptor-null mice are normal, confirming that $\mathrm{AT}_{1 \mathrm{~b}}$ receptor has a negligible role normally. For instance, in the $\mathrm{AT}_{1 \mathrm{a}} \mathrm{R}$ deleted mice, $\mathrm{AT}_{1 \mathrm{~b}}$ receptor substitutes by mediating calcium signaling in vascular smooth muscle cells (VSMC) (Zhu et al., 1998). Animals with both $\mathrm{AT}_{1 \mathrm{a}}$ receptor and $\mathrm{AT}_{1 \mathrm{~b}}$ receptor deletion have increased mortality, impaired growth, hypotension, and marked abnormalities in renal structures (Ito et al., 1995; Sugaya et al., 1995; Chen et al., 1997; Oliverio et al., 1998). There is a complete absence of pressor responses to AngII in the double knockout mice. Kidney functions are mostly affected in $\mathrm{AT}_{1 \mathrm{a}}$ receptor mice, with mild mesangial expansion and juxtaglomerular cell hypertrophy. However, circulating AngII levels are not elevated in these mice. Similarly, vascular tone is not altered through other systems. AngII can elicit an attenuated renal vasoconstriction in $\mathrm{AT}_{1 \mathrm{a}}$ receptor-null mice (Ruan et al., 1999). $\mathrm{AT}_{1 \mathrm{a}}$ receptor knockout causes polyuria and defective urine concentration in mice by reducing vasopressin signaling in the inner medulla (Li et al., 2009c). Structural abnormalities in the vascular system are observed in $\mathrm{AT}_{1 \mathrm{a}}$ receptor mice. Dysfunction in the renal vascular system and change of VSMC proliferation rate, altering the circular mechanical integrity of vessels, and increased synthesis of extracellular matrices are observed (Inokuchi et al., 2001). Ischemiainduced angiogenesis was also impaired, suggesting that $\mathrm{AT}_{1 \mathrm{a}}$ receptor-null condition affects early angiogenesis through inflammatory cell infiltration and angiogenic cytokine expression (Sasaki et al., 2002) and a delay in wound healing (Kurosaka et al., 2009). Reduced differentiation and hypertrophy of adipocytes in $\mathrm{AT}_{1 \mathrm{a}}$ receptor-null mice was also observed (Sasaki et al., 2002). Heart tissue appears to show decrease of risk in the $\mathrm{AT}_{1 \mathrm{a}}$ receptor-null mice, with reduced remodeling of left ventricular wall associated with improved survival after experimental myocardial infarction (Harada et al., 1999). In aortic regurgitation models of $\mathrm{AT}_{1 \mathrm{a}}$ receptor-null mice, long-term survival is improved by attenuating the progression of left ventricule dilatation, hypertrophy, and fibrosis (Nakanishi et al., 2007). Restoring the expression of $\mathrm{AT}_{1 \mathrm{a}}$ receptor in the $\mathrm{C} 1$ neurons of $\mathrm{AT}_{1 \mathrm{a}}$ receptor knockout mice reinstates the sympathoexcitation response to AngII in the rostral ventrolateral medulla (Chen et al., 2010).

Transgenic mice overexpressing $\mathrm{AT}_{1 \mathrm{a}}$ receptor in specific tissues and cells have been developed. Cardiac targeted $\mathrm{AT}_{1 \mathrm{a}}$ receptor overexpression generated a wide spectrum of effects, some of which appear to be strain dependent. Severe cardiac hypertrophy, failure, and death observed at fetal stage is associated with hyperplasia myocytes and heart block (Hein et al., 1997). In rats, similar transgenesis, however, appear to produce normal heart functions. However, cardiac hypertrophy and contractile response to AngII was augmented after volume and pressure overload in these rats (Hoffmann 
et al., 2001). Pressure- or volume-overload causes more pronounced hypertrophy in transgenic rats than in normal rats. Cardiac targeted $\mathrm{AT}_{1}$ receptor overexpression in C57BL/6 mice induces cardiac hypertrophy and remodeling with increased atrial natriuretic factor secretion and interstitial collagen deposition and premature HF. Systolic blood pressure and the heart rate was normal in the transgenic mice (Paradis et al., 2000). Impaired excitation-contraction coupling in heart before the development of cardiac hypertrophy is reported (Rivard et al., 2011). A decrease in myocardial microvessel density after experimental myocardial infarction was observed. Therefore benefit of ARB treatment of myocardial infarction may be due to a stimulatory effect on myocardial angiogenesis (de Boer et al., 2003). Overexpression of $\mathrm{AT}_{1}$ receptor in the cardiac myocytes of angiotensinogen-knockout mice presented spontaneous systolic dysfunction, chamber dilatation, and severe interstitial fibrosis. Treatment with candesartan, an inverse agonist for the $\mathrm{AT}_{1}$ receptor, prevented progressive cardiac remodeling in this model. This proofof-principle study demonstrated that the basal constitutive activity of the $\mathrm{AT}_{1}$ receptor contributes to the cardiac remodeling, in complete absence of AngII, when level of $\mathrm{AT}_{1}$ receptor is increased in the heart (Yasuda et al., 2012).

Overexpression of $\mathrm{AT}_{1 \mathrm{a}}$ receptor restricted to brain enhanced cardiovascular responsiveness to intracerebroventricular injection of AngII without a change in baseline blood pressure. However, with intracerebroventricular injection of losartan to block the central $\mathrm{AT}_{1 \mathrm{a}}$ receptor reduced basal blood pressure, suggesting an enhanced contribution of central $\mathrm{AT}_{1 \mathrm{a}}$ receptor to the maintenance of baseline blood pressure (Lazartigues et al., 2002). Renovascular hypertension in these mice is buffered by increased nitric oxide production in the peripheral vasculature (Lazartigues et al., 2004). An enhanced salt appetite and altered water intake is observed upon brain-selective overexpression of $\mathrm{AT}_{1 \mathrm{a}}$ receptor (Lazartigues et al., 2008).

$\mathrm{AT}_{1}$ receptor overexpression in transgenic rats directed to podocytes induces protein leakage and structural damage to podocytes, progressing to focal segmental glomerulosclerosis (Hoffmann et al., 2004). Mice overexpressing a constitutively active $\mathrm{AT}_{1}$ receptor transgene in renal proximal tubule caused increased baseline blood pressure. Depletion of endogenous $\mathrm{AT}_{1 \mathrm{a}}$ receptor in the proximal tubule reduced blood pressure. However, there was no difference in the blood pressure response to a pressor dose of AngII in either experimental model, suggesting that the $\mathrm{AT}_{1 \mathrm{a}}$ receptor in the renal proximal tubule is a regulator of systemic blood pressure under baseline conditions ( $\mathrm{Li}$ et al., 2011).

Transgenic overexpression of the constitutively active $\mathrm{N} 111 \mathrm{G}$ mutant $\mathrm{AT}_{1}$ receptor restricted to vascular endothelium significantly reduced the pressor response of carotid artery to acute infusion of AngII, resulting in hypotension and bradycardia (Ramchandran et al., 2006). Increased nitric oxide synthase expression in the endothelial cells seems to increase NO in blood and moderate response of smooth muscle cells to AngII. Gene knock-in expression of the constitutively active N111S mutant of $\mathrm{AT}_{1}$ receptor with a C-terminal deletion produced long-lasting pressor response to infused AngII and a moderate and stable increase in blood pressure (Billet et al., 2007). These mice develop diastolic dysfunction without developing significant cardiac hypertrophy but show progressive renal and cardiac fibrosis. Overexpression of the constitutively active $\mathrm{N} 111 \mathrm{G}$ mutant of $\mathrm{AT}_{1}$ receptor in cardiac myocytes produced enhanced myocyte growth from the onset of adolescence associated with cardiac hypertrophy in the adult without progressing to pathologic remodeling or HF (Ainscough et al., 2009). However, AngIV peptide infusion induced adverse ventricular remodeling within 4 weeks characterized by increased interstitial fibrosis, dilatation of the left ventricle, and impaired cardiac function.

Transgenesis for inhibition of the RAS at a genetic level, which involves an antisense targeting $\mathrm{AT}_{1}$ receptor, has been proposed as therapy for chronic control of blood pressure. A retrovirus-based delivery of $\mathrm{AT}_{1}$ receptor antisense was reported to prevent hypertension in the spontaneously hypertensive rats (Reaves et al., 2000; Wang et al., 2000b) and protects normotensive rats from developing AngII-infused hypertension (Pachori et al., 2000). Intracerebroventricular injections of antisense oligonucleotides targeting $\mathrm{AT}_{1}$ receptor in the brain decreased the blood pressure in chronic 2-kidney, 1-clip hypertensive rats (Kagiyama et al., 2001). Antisense inhibition of the $\mathrm{AT}_{1}$ receptor in human pulmonary artery smooth muscle cells has powerful inhibitory effects on AngII-induced migration, proliferation of, and promotion of apoptosis (Tu et al., 2005). These studies suggest that antisense inhibition targeting to $\mathrm{AT}_{1}$ receptor has therapeutic potential for the treatment of vascular diseases, including hypertension and hypertension-associated cardiac and vascular pathophysiology.

\section{Genetic Polymorphism}

The AGTR1 gene is highly polymorphic (Duncan et al., 2001; Mottl et al., 2008). In particular, nucleotide $1166 \mathrm{~A} / \mathrm{C}$ transversion in the $3^{\prime}$ untranslated region of the gene (Bonnardeaux et al., 1994) has been associated with essential hypertension, increased aortic stiffness (Benetos et al., 1995), and myocardial infarction (Berge et al., 1997), with large interethnic, age, and sex differences in the frequencies. At least 50 single nucleotide polymorphisms (SNPs) have been described, among which nine of them are in the functional promoter region of the gene having the potential to influence $\mathrm{AT}_{1}$ receptor gene expression (Erdmann et al., 1999). The significance of $\mathrm{AT}_{1}$ receptor polymorphisms in 
essential hypertension remains controversial (Griendling et al., 1996; Luft, 2004). Association of A1166C with increased sensitivity for AngII was reported in hypertensive patients on a high-salt diet (Spiering et al., 2000). A1166C polymorphism was associated with hypertensionrelated impairment of renal function (Buraczynska et al., 2002; Coll et al., 2003). A1166C was associated with enhanced vasoconstriction by AngII in isolated human arteries (van Geel et al., 2000). The A1166C polymorphism may increase the risk of coronary heart disease in patients with familial hypercholesterolemia (Wierzbicki et al., 2000). The association of A1166C polymorphism with losartan treatment in hemodynamic response measurement should be important for further research to understand the individual responses to a variety of $\mathrm{AT}_{1}$ receptor blockers (ARBs) and develop personalized antihypertensive therapy (Baudin, 2002).

Naturally occurring amino acid variations in $\mathrm{hAT}_{1}$ receptor are reported in genome databases (http://www. uniprot.org/uniprot/P30556). These include miss-sense variations, L48V, A163T, L222V, A244S, T282M, C289W, T336P, P341H. Of these miss-sense variations, A163T, $\mathrm{T} 282 \mathrm{M}$, and C289W may directly affect ligand binding, and L48V, L222V, and A244S may indirectly influence ligand binding or signaling by $\mathrm{AT}_{1}$ receptor (see Fig. 5). Variant residues, $\mathrm{T} 336 \mathrm{P}$ and $\mathrm{P} 341 \mathrm{H}$ are located in the C-terminal tail that is not included in the crystalized $\mathrm{AT}_{1}$ receptor. However, these residues are known to be phosphorylated, an event that is necessary for $\beta$-arrestin recruitment to $\mathrm{AT}_{1}$ receptor and subsequent receptor trafficking to endosomes. The $\mathrm{T} 282 \mathrm{M}$ variant is linked

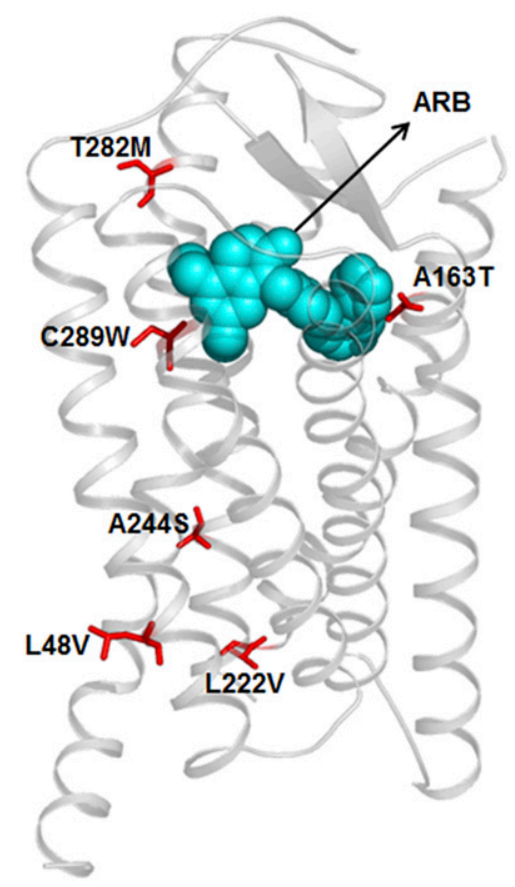

Fig. 5. Single residue variations of $\mathrm{hAT}_{1}$ receptor in population. Location of reported variant residues are shown in an ARB bound threedimensional model of the receptor. to renal tubular digenesis syndrome by an unknown mechanism (Gribouval et al., 2005) and the Thr163 variant lowered affinity for losartan (Arsenault et al., 2010b). The $\mathrm{AT}_{1}$ receptor crystal structure indicated that $14 \%$ of Ala $163^{\mathrm{TM} 4}$ side-chain surface interacted with ARBs. Cys $289^{\mathrm{TM} 7}$ faces ARB binding pocket but does not interact with ARBs, and Thr282 $2^{\mathrm{TM} 7}$ is not in the ARB pocket. Mutagenesis studies have shown that residues located closely to the binding site reduce the affinity of ARBs and AngII but not Thr282 $2^{\text {TM7 }}$ and Cys289 ${ }^{\text {TM7 }}$, suggesting that variants may not directly alter ligand-receptor interactions (Zhang et al., 2015). However, the variant residues in humans are different from those evaluated in mutagenesis studies; therefore variant residue effects on ligand binding need to be determined through experiments. Phenotypic effects of other variant residues are difficult to predict, but may affect AngII and antihypertensive response in individuals carrying these variations.

\section{E. Signaling}

Over the past 15 years, the $\mathrm{AT}_{1}$ receptor signaling has been studied in great detail (see Fig. 6) to understand mechanism of regulation of vasoconstriction, sodium reabsorption, cell proliferation, extracellular matrix formation, inflammation, and oxidative stress by RAS and how ARBs might intercept signaling during pathology (De Gasparo, 2002; Lefkowitz and Shenoy, 2005; Rajagopal et al., 2005; Hunyady and Catt, 2006; Mehta and Griendling, 2007; Oliveira et al., 2007; Oro et al., 2007; Violin and Lefkowitz, 2007; Aplin et al., 2009; Lyngso et al., 2009). $\mathrm{AT}_{1}$ receptor is the lead example for establishing a novel cell signaling principle that a single GPCR ligand can activate multiple signaling pathways both dependent and independent of heterotrimeric G-proteins with differing efficacies (Kenakin, 2001; Lefkowitz and Shenoy, 2005; Violin and Lefkowitz, 2007; Patel et al., 2010).

The traditionally portrayed signaling mechanism of the $\mathrm{AT}_{1}$ receptor is dependent on heterotrimeric $\mathrm{G}$ proteins (Hunyady and Catt, 2006). In addition to coupling with the heterotrimeric G-proteins, AngII activates both nonreceptor and receptor tyrosine kinases (Ishida et al., 1995; Sadoshima and Izumo, 1996; Bernstein et al., 1998; Eguchi et al., 1998; Sadoshima, 1998; Heeneman et al., 2000; Saito and Berk, 2001; Higuchi et al., 2007). These interactions orchestrate pleotropic signaling in cells that include enzymes, adapter proteins, transcription factors, and small GTP binding proteins and downstream kinases, accounting for a wide spectrum of responses to AngII (Marrero et al., 1995b; Venema et al., 1998b).

1. G-protein-Mediated Signaling. Multiple heterotrimeric G-proteins interact with the $\mathrm{AT}_{1}$ receptor, including $\mathrm{G}_{\mathrm{q} / 11}, \mathrm{G}_{\mathrm{i}}, \mathrm{G}_{12}$, and $\mathrm{G}_{13}$, leading to activation of downstream effectors including phospholipase C (PLC), phospholipase A, and phospholipase D (Shirai et al., 1995; Ushio-Fukai et al., 1999a; Higuchi et al., 2007). 


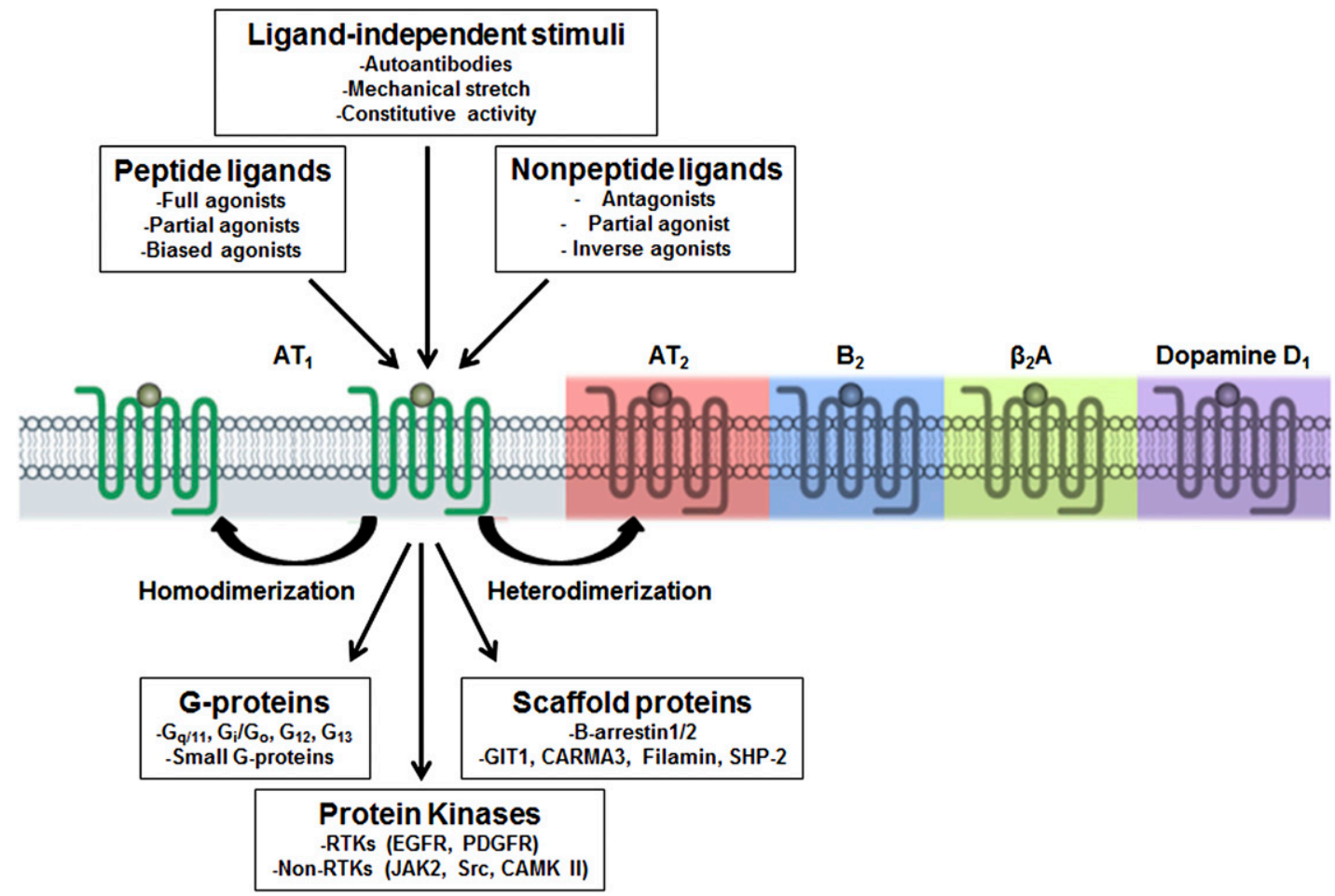

Fig. 6. Schematic representation of $\mathrm{AT}_{1}$ receptor as a pluridimensional signal transducer. Discoveries made in the past 15 years suggest that $\mathrm{AT}_{1}$ receptor as a transmembrane transducer capable of sensing multiple modes of stimuli and elicit diverse responses as shown.

The $\mathrm{AT}_{1}$ receptor- $\mathrm{G}_{\mathrm{q} / 11}$-phospholipase $\mathrm{C} \beta(\mathrm{PLC} \beta)$ coupling results in inositol triphosphates (IP3) and diacylglycerol (DAG) (Yusuf et al., 2000) signals. IP3 causes release of $\mathrm{Ca}^{2+}$ from the intracellular store. Intracellular $\mathrm{Ca}^{2+}$ cycling is the primary trigger for excitation-contraction of both cardiac and vascular myocytes. In addition, cytosolic $\mathrm{Ca}^{2+}$ does trigger intracellular signaling through calcineurin/nuclear factor of activated in T-cells or the calcium/calmodulin-dependent protein kinase II (CamK II) cascades, which are relevant to hypertrophy (Heineke and Molkentin, 2006). Histone deacetylase-5 has emerged as an important substrate of CamK II. A scaffold protein, GIT1, mediates AngII-induced VSMC gene transcription via CamK II-dependent phosphorylation and activation of HDAC5 (Pang et al., 2008). DAG activates PKC (Vallega et al., 1988), which also contributes to the vasoconstrictive and growth promoting effects of AngII. Phospholipase D-mediated phosphatidylcholine to choline and phosphatidic acid production is considered a second wave of signal by AngII activated $\mathrm{AT}_{1}$ receptor. $\mathrm{PA}$ is rapidly converted to DAG, leading to sustained muscle contraction (Mehta and Griendling, 2007). $\mathrm{AT}_{1}$ receptor causes activation of phospholipase A2 by phosphorylation and production of arachidonic acid and its metabolites. This signal is important in maintaining a balance between vasoconstriction and vasodilation in various vascular beds (Sarkis et al., 2004; Campbell et al., 1996) and $\mathrm{NAD}(\mathrm{P}) \mathrm{H}$ oxidation in VSMC (Griendling et al., 2000).
The $\mathrm{G}_{\beta \gamma}$ subunits released upon $\mathrm{AT}_{1}$ receptor activation have been shown to activate tyrosine kinases such as SHC, pp60 ${ }^{\text {c-src }}$, and JAK2 (Gutkind, 1998; Gschwind et al., 2001; Kranenburg and Moolenaar, 2001; Luchtefeld et al., 2001), leading to downstream phosphatidylinositol 3'-kinase $\gamma\left(\right.$ PI3K $\left._{\gamma}\right)$ activation (Lopez-Ilasaca et al., 1997). AngII-induced activation of $\mathrm{AT}_{1}$ receptor specifically augmented $\mathrm{G}_{\beta 2}$ levels in the nucleus, where $\mathrm{G}_{\beta 2}$ interacts with specific nucleosome core histones and specific chromatin bound transcription factors such as myocyte enhancer factor 2 and thereby regulates functional gene networks (Bhatnagar et al., 2013).

2. G-Protein-Independent $\beta$-Arrestin-Mediated Signaling. Multiple lines of observations led to the discovery that $\mathrm{AT}_{1}$ receptor can directly recruit $\beta$-arrestin and mediate ERK1/2 signaling without G-protein activation (Holloway et al., 2002; Gaborik et al., 2003; Wei et al., 2003; Ahn et al., 2004a; Lee et al., 2008). The classic concept is that $\beta$-arrestin terminates G-protein signaling by blocking the receptor and stimulating receptor desensitization and endocytosis. However, $\beta$-arrestinbound receptor can engage in G-protein-independent signaling, leading to distinct cellular responses (DeWire et al., 2007; Violin et al., 2013). This mode of signaling is recognized in a number of in vitro and in vivo settings; $\mathrm{AT}_{1}$ receptor recruited $\beta$-arrestin $1 / 2$ engage a wide range of cellular responses.

The $\mathrm{AT}_{1}$ receptor coupled $\beta$-arrestin mediates delayed ERK1/2 signaling confined to the cytoplasm for prolonged time $\left(t_{1 / 2}>25 \mathrm{~min}\right)$. In contrast, both nuclear 
and cytoplasmic localization of activated ERK is Gprotein-mediated (Gaborik et al., 2003; Tohgo et al., 2003; Ahn et al., 2004a). The $\mathrm{AT}_{1}$ receptor- $\beta$-arrestin complex inhibits ERK-dependent transcription by binding to phospho-ERK and its retention in the cytosol (Tohgo et al., 2002). The cytoplasmic pool of ERK1/2 failed to phosphorylate the transcription factor Elk-1 and increase transcription of the immediate-early gene c-Fos (Tohgo et al., 2002; Aplin et al., 2007b). The $\beta$-arrestin2-dependent ERK activation may be responsible for distinct physiologic endpoints (Wei et al., 2004), such as increased survival of cardiac myocytes without entailing pathogenic myocyte hypertrophy. The beneficial effects of the $\beta$-arrestin-dependent pathway on enhancing cardiomyocyte survival was confirmed in a physiologic study in which a transgenic mouse with cardiac-specific overexpression of $\mathrm{AT}_{1}$ receptor with second intracellular loop mutations that prevent G-protein coupling showed marked ventricular dilation and eccentric hypertrophy accompanied by diminished cardiomyocyte apoptosis in comparison with mice overexpressing a wild-type $\mathrm{AT}_{1}$ receptor (Zhai et al., 2005).

Mechanistic details of activation of $\beta$-arrestin signaling by $\mathrm{AT}_{1}$ receptor are emerging. $\mathrm{AT}_{1}$ receptor can bind both $\beta$-arrestin 1 and 2 (Oakley et al., 2000). Ahn et al. (2004b) demonstrated that physiologic levels of $\beta$ arrestin 1 might antagonize $\beta$-arrestin $2-$ mediated ERK activation. Zimmerman et al. (2012) showed that AngII analogs selectively promoted $\beta$-arrestin-dependent effects in VMSCs. A mass spectrometry-based proteomics approach discovered that $\beta$-arrestin interacts with several proteins selectively after stimulation of the $\mathrm{AT}_{1}$ receptor, indicating its potential for signaling (Xiao et al., 2007). In another study, activation by the $\beta$-arrestin biased ligand SII-AngII showed that unique phosphoproteins are activated (Christensen et al., 2010; Xiao et al., 2010). There are 34 differentially phosphorylated proteins, of which 16 were unique to SII-AngII and 8 were unique to AngII stimulation. Bioinformatics analysis of phosphorylated sites on these proteins identified downstream protein kinases activated by the $\mathrm{AT}_{1}$ receptor- $\beta$-arrestin signaling (Kendall et al., 2011; Bogebo et al., 2014).

3. Reactive Oxygen Species Signaling. AngII is a potent mediator of oxidative stress and oxidant signaling (Ushio-Fukai et al., 1999b; Taniyama and Griendling, 2003; Yan et al., 2003a; Touyz, 2004). AngII activates membrane $\mathrm{NAD}(\mathrm{P}) \mathrm{H}$ oxidase mainly via $\mathrm{AT}_{1}$ receptor and $\mathrm{PKC}$ to produce reactive oxygen species (ROS) like superoxide and hydrogen peroxide $\left(\mathrm{H}_{2} \mathrm{O}_{2}\right)$ (Rajagopalan et al., 1996; Ushio-Fukai et al., 1996; Zafari et al., 1998; Griendling et al., 2000; Seshiah et al., 2002; Touyz et al., 2005). ROS are involved in many pleiotropic effects of AngII such as activation of signaling molecules (e.g., c-Src, EGFR, p38MAPK, Akt) and transcription factors [e.g., nuclear factor $\kappa \mathrm{B}(\mathrm{NF} \kappa \mathrm{B}), \mathrm{NF}-\kappa \mathrm{B}, \mathrm{AP}-1$, Nrf2], which are involved in atherosclerosis pathology (Sen and Packer, 1996; Chen et al., 2006; Papaiahgari et al., 2006; Wu et al., 2005). In endothelial cells, a low amount of superoxide production by eNOS maintains a supply of NO, contributing to vasodilation and vascular health. In disease state, eNOS is uncoupled, leading to excessive superoxide that reacts with NO to form peroxynitrite, a toxic radical, which directly contributes to disease (Schena et al., 1999; Zhao et al., 2005a; Taguchi et al., 2011).

4. Nonreceptor Type Tyrosine Kinase Signaling. Nonreceptor tyrosine kinases associate with $\mathrm{AT}_{1}$ receptor and target several intracellular proteins for phosphorylation. C-Src is a key player in AngII-mediated cellular effects. C-Src tyrosine kinase activity is also activated by ROS and it is involved in sustained calcium release (Sadoshima, 1998). Src is activated by AngII in an $\mathrm{AT}_{1}$ receptor mutant lacking G-protein coupling ability, indicating that the carboxyl terminus of the $\mathrm{AT}_{1}$ receptor is required for activation (Seta et al., 2002) because C-terminal truncation (310-359) abolished Src activation. VSMC growth is mediated by hyperactivation of c-Src-ERK1/2-dependent pathways by $\mathrm{AT}_{1}$ receptor, leading to c-fos and AP-1 DNA-binding activity (Touyz et al., 2001b). AngII-stimulation of human monocyte migration is c-Src-dependent (Ishida et al., 1999) formation of focal adhesion complexes in the actin cytoskeleton (Polte et al., 1994). $\mathrm{AT}_{1}$ receptor induces tyrosine phosphorylation of FAK, which then form a complex with Pyk2, p130Cas, paxillin, and talin, all of which interact to enable activation of cytoskeletal proteins facilitating adhesion of cells to extracellular matrix, and regulation of cell shape and movement (Leduc and Meloche, 1995; Sabe et al., 1997; Cary et al., 1998; Sayeski et al., 1998; Eguchi et al., 1999; Kintscher et al., 2001). Pyk2 is another kinase activated in response to $\mathrm{AT}_{1}$ receptor and has been implicated in the regulation of ion channels, cellular adhesion, cell growth, and mitogenic and hypertrophic reactions (Sabri et al., 1998; Tang et al., 2000; Taniyama et al., 2003).

$\mathrm{AT}_{1}$ receptor activates the JAK/STAT pathway via scaffolding by Src homology phosphatase-2 (SHP-2), a tyrosine phosphatase. The conserved YIPP motif in the $\mathrm{AT}_{1}$ receptor interacts with SHP-2 and helps docking JAK2 and stimulates JAK2 phosphorylation at $\mathrm{Tyr}^{1007} / \mathrm{Tyr}^{1008}$ (Marrero et al., 1995a; Doan et al., 2001; Frank et al., 2002; Godeny et al., 2007). Upon activation of JAK2 by $\mathrm{AT}_{1}$ receptor, STAT proteins are activated in order to mediate gene transcription of early growth response genes, such as c-fos and c-myc (Berk and Corson, 1997; Ishida et al., 1999; Luttrell et al., 1999; Madamanchi et al., 2001). Another tyrosine phosphatase, SHP-1, causes JAK2 dephosphorylation and termination of the AngII-induced JAK/STAT signaling (Marrero et al., 1998). A calcium/PYK2 and PKC pathway also exists for JAK2 activation in VSMCs (Frank et al., 2002). $\mathrm{An}_{\mathrm{AT}}$ receptor mutant with tyrosine 292, 302, 312, 319, and 339 mutated to phenylalanine uncoupled from 
G-proteins was found to still activate tyrosine kinases and phospho-STAT1 signaling, indicating G-protein independence of these signals. JAK2 activation induces the expression of SOCS-3, which, in turn, blocks further activation of the pathway and consequently leads to desensitization of this signaling path (Torsoni et al., 2004).

5. Activation of Small G-protein Signaling. AngII also activates family of small G-proteins, such as Ras, Rho, and Rac through the $\mathrm{AT}_{1}$ receptor (Ohtsu et al., 2006c), which regulate the MAPK cascades in cardiovascular remodeling induced by AngII. Ras is activated in both cardiac myocytes and VSMCs (Eguchi et al., 1996; Sadoshima and Izumo, 1996) by SHP-2 phosphorylation, which leads to Shc/Grb2 complex and recruitment of the guanine nucleotide exchange factor, son of sevenless. Subsequently son of sevenless activates the Ras/Raf/MEK/ERK1/2 pathway and c-fos transcriptional activity (Sugden and Clerk, 1997; Ohtsu et al., 2006c). PKC can also associate with Ras and activate ERK1/2 (Liao et al., 1996; Liao et al., 1997). Accumulating evidence suggests that the Rho/ ROCK (Rho-kinase) pathway is crucial for AngIIinduced remodeling of vasculature (Aoki et al., 1998). Rho pathways are involved in cell migration (Seko et al., 2003). Rac is implicated in activation of p21activated kinase 1 by AngII in VSMCs, which subsequently mediates JNK activation and hypertrophy (Seko et al., 2003; Woolfolk et al., 2005; Ohtsu et al., 2006a). Rho pathway is involved in the $\mathrm{Ca}^{2+}$ sensitization of smooth muscle contraction, pre-myofibril formation, and expression of atrial natriuretic factor in cardiac myocytes. Rac is an important component of the reduced NADPH oxidase complex to produce ROS by AngII in VSMCs (Gregg et al., 2003). ERK1/2, JNK, and p38MAPK activated downstream of $\mathrm{AT}_{1}$ receptorNADPH pathway are implicated in VSMC differentiation, proliferation, and migration (Sugden and Clerk, 1997; Taniyama et al., 2004), as well as in fibrosis and target-organ damage (Ishida et al., 1998; Ishida et al., 1999). The phosphatase MAPK phosphatase-1 (MKP-1) serves as a negative feedback control, inactivating ERK1/2 (Bokemeyer et al., 1998).

6. Transactivation of Receptor Tyrosine Kinase Signaling. $\mathrm{AT}_{1}$ receptor-induced transactivation of platelet derived growth factor receptor (PDGFR) modulates cell growth and migration (Heeneman et al., 2000; Suzuki and Eguchi, 2006) in VSMCs and mesangial cells (Linseman et al., 1995; Mondorf et al., 2000). This response is blocked by losartan and other ARBs. Upon acute AngII infusion, activation of PDGFR occurs in the vasculature of mice and rats. ACE-inhibitor infusion reduced aortic PDGFR phosphorylation and ERK activity (Kim et al., 2000), implicating PDGFR as downstream modulator of hypertensive vascular remodeling in vivo (Linseman et al., 1995; Heeneman et al., 2000; Mondorf et al., 2000; Gao et al., 2006).
AngII infusion also leads to activation of epidermal growth factor receptor (Zhang et al., 2009) in the vasculature (Kim et al., 2000). A major mechanism by which AngII influences growth-signaling pathways is through transactivation of EGFR by $\mathrm{AT}_{1}$ receptor. AngII-induced renal deterioration involves EGFR transactivation mediated by ADAM17 (Lautrette et al., 2005) and second messengers such as $\mathrm{Ca}^{2+}$ and ROS (DiazRodriguez et al., 2002; Mori et al., 2003; Seals and Courtneidge, 2003; Fischer et al., 2004; Tanaka et al., 2004; Mifune et al., 2005; Ohtsu et al., 2006a; Zhang et al., 2006). $\mathrm{AT}_{1}$ receptor activation enhances the release of heparin-binding epidermal growth factor, which is dependent on A Disintegrin and Metalloproteinase (ADAM) family metalloproteinases (ADAM17) and Src (Andreev et al., 2001; Eguchi et al., 2001; UchiyamaTanaka et al., 2001; Schafer et al., 2004; Shah et al., 2004; Blobel, 2005; Mifune et al., 2005; Ohtsu et al., 2006a,b). Furthermore, dominant-negative ADAM17 mutant distinctly inhibited VSMC hypertrophy, which was stimulated by AngII (Ohtsu et al., 2006b). Heparin-binding epidermal growth factor activates EGFRs, allowing autophosphorylation on tyrosine (Prenzel et al., 1999). AngII-induced EGFR transactivation requires ROS and upstream kinases, such as c-Src, c-Abl, or Pyk2 (Dikic et al., 1996; Bokemeyer et al., 2000; Seshiah et al., 2002; Gratton et al., 2004) and leads to activation of the Ras/ Raf/ERK pathway. AngII activation of Akt/PKB, p70S6K, and p38MAPK and induction of c-Fos leading to growth and migration of VSMCs, survival, and remodeling are mediated by EGFR (Che and Carmines, 2002; Seshiah et al., 2002; Suzuki et al., 2005).

A $\beta$-arrestin-dependent mechanism for transactivation of EGFR by $\mathrm{AT}_{1}$ receptor has been reported (Kim et al., 2009). Calcium-independent AngII pathways also cause EGFR transactivation (Murasawa et al., 1998; Wang et al., 2000a) and the biased AngII analog [Sar ${ }^{1}$, $\left.\mathrm{Ile}^{4}, \mathrm{Ile}^{8}\right]$ AngII activated $\mathrm{G}_{\mathrm{q}}$-independent EGFR signaling in human coronary artery smooth muscle cells (Miura et al., 2004). It was also reported that phosphorylation of tyrosine 319 and the YIPP motif of the $\mathrm{AT}_{1}$ receptor is required for transactivation of EGFR (Seta and Sadoshima, 2003; Zhai et al., 2006). Cardiacspecific overexpression of an $\mathrm{AT}_{1}$ receptor with a mutation in the YIPP motif (Tg-Y319F) did not cause cardiac hypertrophy in transgenic mice, and expression of fetal-type genes was significantly lower in these mice. Infusion of AngII failed to induce hypertrophy in TgY319F mice, also significantly less apoptosis and fibrosis was reported (Zhai et al., 2006; Smith et al., 2011). EGFR activation is reported to be necessary for AngII-mediated hypertension and left ventricular hypertrophy (Ahmad et al., 2009).

7. Signaling through $A T_{1}$ Receptor Interacting Scaffold Proteins. $\mathrm{AT}_{1}$ receptor signaling occurs through the recruitment of scaffolding regulatory proteins. The carboxyl-terminal cytoplasmic region of the $\mathrm{AT}_{1}$ receptor 
recruits different proteins to regulate different aspects of $\mathrm{AT}_{1}$ receptor physiology (Mogi et al., 2009; Horiuchi et al., 2012). $\mathrm{AT}_{1}$ receptor-associated protein (ATRAP1) is a transmembrane protein expressed in various tissues including the kidney, aorta, heart, lung, testis, and at a lower level in the lung, liver, spleen, and brain. It interacts specifically with the C-terminal tail and enhances AngII-induced internalization of $\mathrm{AT}_{1}$ receptor (Daviet et al., 1999; Cui et al., 2000; Guo et al., 2003; Oshita et al., 2006; Azuma et al., 2007). ATRAP1 is a negative regulator of classic G-protein signaling by $\mathrm{AT}_{1}$ receptor (Lopez-Ilasaca et al., 2003; Tamura et al., 2007) and VSMC growth (Cui et al., 2000) and cardiomyocyte hypertrophy (Tanaka et al., 2005). ATRAP1 prevents VSMC senescence (Wislez et al., 1998; Guo et al., 2005). ATRAP1 transgenic mice exhibited decrease in cardiac hypertrophy, neointima formation, inflammatory response, and NADPH oxidase activity in the injured artery (Oshita et al., 2006; Wakui and Tamura, 2012). In contrast, ATRAP1-deficient (ATRAP1-/-) mice showed increased mean systolic blood pressure and plasma volume, which was associated with increased surface expression of $\mathrm{AT}_{1}$ receptors in the renal cortex and increased proximal tubular function (Oppermann et al., 2010). Kidney-specific ATRAP1 transgenic mice exhibit hypertension and renal hypertrophy and failure (Oppermann et al., 2010), suggesting that renal ATRAP1 plays an important role in regulating intrarenal RAS. Mechanistic aspects of $\mathrm{AT}_{1}$ receptor interaction with ATRAP1 and transfer of signal are not clear at this time (Cook et al., 2008).

8. Mechanical Stretch. $\mathrm{AT}_{1}$ receptors were identified as mechanosensors in the myocardium by Issei Komuro's team by demonstrating agonist-independent activation of $\mathrm{AT}_{1}$ receptor when stretch-stress is applied (Hunyady and Turu, 2004; Yasuda et al., 2008a; Mederos y Schnitzler et al., 2011). Mechanical stretch activated ERKs in the cardiomyocytes prepared from both neonatal and adult angiotensinogen-deficient mice (Zou et al., 2004), which could be inhibited by inverse agonist ARBs, such as candesartan. Mechanical stretch induces Janus kinase 2 and translocation of G-proteins into the cytosol (Zou et al., 2004; Yasuda et al., 2008a). The conformational changes in mechanically activated $\mathrm{AT}_{1}$ receptor have been mapped (Shyu et al., 2001; Karnik et al., 2003; Yasuda et al., 2008b).

Sadoshima et al. (1993) initially reported that mechanical stretch causes secretion of AngII from cytoplasmic storage granules in cultured cardiac myocytes and that stretch-induced hypertrophic responses are completely dependent on the secreted AngII. However, several studies later showed that AngII partly mediates mechanical stress-induced hypertrophic responses (Yamazaki et al., 1995; Kijima et al., 1996). Therefore, mechanical stretch seems to directly activate unique intracellular signaling molecules. Yasuda et al. (2008b) showed that cell stretch leads to activation of the $\mathrm{AT}_{1}$ receptor, which could be suppressed by candesartan, an inverse agonist. Thus, mechanical stress can directly change the conformation of the $\mathrm{AT}_{1}$ receptor to increase the receptor's basal activity. This was followed by Zou et al. (2004), demonstrating that the $\mathrm{AT}_{1}$ receptor can be activated by mechanical stress inducing cardiac hypertrophy in vivo in an AGT-null background.

Stretch-induced activation of the $\mathrm{AT}_{1}$ receptor protects cells against induced apoptosis involving $\mathrm{PKB} / \mathrm{Akt}$ signaling (Kippenberger et al., 2005). Mechanical stretch potentiates AngII-induced VSMCs proliferation in spontaneously hypertensive rat through an $\mathrm{AT}_{1}$ receptor/ EGFR/ERK-dependent pathway. These findings may provide new insights into growth-promoting mechanisms in vasculature in a hypertensive state (Liu et al., 2010). Mechanical stretch triggered an $\mathrm{AT}_{1}$ receptordependent conformational change in $\beta$-arrestin similar to that induced by a $\beta$-arrestin-biased ligand (Rakesh et al., 2010). These findings were unique to the $\mathrm{AT}_{1}$ receptor (and not seen with the $\beta 1$ adrenergic receptors) and suggest that $\mathrm{AT}_{1}$ receptor is able to sense membrane stretch and transmit the activated receptor signal to $\beta$-arrestin.

9. Signaling through Heterodimerization. The current view is that homo- and heterodimer formation of GPCRs could be important for some of the receptor functions (Lyngso et al., 2009). For example, the $\mathrm{AT}_{1}$ receptor dimerizes with the bradykinin $\mathrm{B} 2$ receptor (Fig. 6), which enhances AngII signaling (AbdAlla et al., 2000, 2001b, 2005), contributing to AngII hypersensitivity in women with preeclampsia (AbdAlla et al., 2000, 2001b). The arrestin-biased ligand $\left[\mathrm{Sar}^{1}, \mathrm{Ile}^{4}, \mathrm{Ile}^{8}\right] \mathrm{AngII}$ negatively regulates $\mathrm{AT}_{1}$ receptor-B2R heterodimers by promoting sequestration of $\mathrm{AT}_{1}$ receptor-B2R heterodimers (Wilson et al., 2013). Heterodimerization of $\mathrm{AT}_{1}$ receptor with the MAS receptor and $\mathrm{AT}_{2}$ receptor decreases $\mathrm{AT}_{1}$ receptor-specific signaling (AbdAlla et al., 2001a; Kostenis et al., 2005; Canals et al., 2006; Santos et al., 2007). The $\mathrm{AT}_{1}$ receptor can also form complexes with the $\beta 2$ adrenergic receptors, and it is possible to effectively block dual receptor signaling using only a single receptor antagonist (Barki-Harrington et al., 2003). The $\mathrm{AT}_{1}$ receptor also coimmunoprecipitates with the epidermal growth factor receptor (EGFR); dopamine D1, D3, and D5; and the endothelin B receptors (Zeng et al., 2003a,b, 2005a,b, 2006; Olivares-Reyes et al., 2005).

Evidence that $\mathrm{AT}_{1}$ receptors can form dimers is supported by BRET analysis, suggesting homo- or oligomeric complexes in living cells that are unaffected by both agonists and antagonists (Hansen et al., 2004). Coexpression of signaling-deficient mutants results in functional receptors. $\mathrm{AT}_{1}$ receptor wild-type G-protein coupling was diminished when coexpressed with defective mutant receptors, indicating "cross-inhibitory" association (Karip et al., 2007). Covalently crosslinked homodimer formation for $\mathrm{AT}_{1}$ receptor is reported in isolated monocytes (AbdAlla et al., 2004) induced by 
factor XIIIA transglutaminase involving $\mathrm{Gln}^{315}$ in the carboxyl-terminal tail of the $\mathrm{AT}_{1}$ receptor. Hypertensive patients have increased homodimer levels (AbdAlla et al., 2004; Ogawa and Glass, 2004). Aldosterone produces a nongenomic endothelium-independent vasoconstrictor effect by enhancing intracellular transglutaminase activity and presumably inducing $\mathrm{AT}_{1}$ receptor dimer formation in mesenteric arterioles perhaps due to transglutaminase-induced $\mathrm{AT}_{1}$ receptor dimer formation (Yamada et al., 2008).

10. AngiotensinII Type 1 Receptor Signaling by Phosphorylation, Desensitization, and Internalization. G-protein signaling by activated $\mathrm{AT}_{1}$ receptor is accompanied by rapid phosphorylation and internalization of $\mathrm{AT}_{1}$ receptor (Thomas, 1999; Hunyady et al., 2000; Guo et al., 2001; Thomas and Qian, 2003). Defects in desensitization are implicated in vascular diseases; for example, hypertensive rats overexpress GRK 5, altering AngII responsiveness (Ishizaka et al., 1997). AngIIinduced desensitization of $\mathrm{AT}_{1}$ receptor is dependent on carboxyl-terminal residues 329-347 (Conchon et al., 1998). This region is Ser and Thr rich, and phosphorylation of these residues plays a key role in the desensitization of $\mathrm{AT}_{1}$ receptor responses. AngII-induced phosphorylation of the $\mathrm{AT}_{1}$ receptor is mediated by both PKC and G-protein-coupled receptor kinases (GRK), mainly GRK2 and GRK5 (Oppermann et al., 1996a,b; Smith et al., 1998a; Qian et al., 1999). Mutation of the key serine and threonine residues in 332-338 region significantly inhibits $\mathrm{AT}_{1}$ receptor internalization (Hunyady et al., 1994; Thomas et al., 1995, 1998; Smith et al., 1998b; Qian et al., 2001). Mutating hydrophobic residues in helix VIII in $\mathrm{C}$ terminus also inhibits $\mathrm{AT}_{1}$ receptor internalization (Thomas et al., 1995). A diacidic motif of $\mathrm{Asp}^{236}-\mathrm{Asp}^{237}$ in the ICL3 of the $\mathrm{AT}_{1}$ receptor is required for optimal AngII-induced phosphorylation of $\mathrm{AT}_{1}$ receptor by GRKs and internalization (Olivares-Reyes et al., 2001). Complete deletion of the cytoplasmic tail inhibits internalization of $\mathrm{AT}_{1}$ receptor (Chaki et al., 1994; Hunyady et al., 1994; Balmforth et al., 1995), and an STL motif ( $\mathrm{Ser}^{335}$ $\mathrm{Thr}^{336}-\mathrm{Leu}^{337}$ ) in this region plays a critical role but also requires residues $\mathrm{Leu}^{316}$ and $\mathrm{Tyr}^{319}$ (Hunyady et al., 1994; Thomas et al., 1995).

Internalization of the $\mathrm{AT}_{1}$ receptor follows both $\beta$-arrestin-dependent and -independent mechanisms (Lefkowitz, 1998; Somsel Rodman and WandingerNess, 2000; Kim et al., 2005a). Alanine substitution for $\mathrm{Thr}^{332}$, $\mathrm{Ser}^{335}$, $\mathrm{Thr}^{336}$, and $\mathrm{Ser}^{338}$ preclude agonistinduced $\beta$-arrestin recruitment by $\mathrm{AT}_{1}$ receptor and attenuated internalization (Luttrell et al., 2001; Qian et al., 2001; Kule et al., 2004). Dramatically reduced $\mathrm{AT}_{1}$ receptor internalization was observed in mouse embryonic fibroblasts lacking both $\beta$-arrestin 1 and $\beta$-arrestin 2 (Kohout et al., 2001). $\beta$-Arrestins target $\mathrm{AT}_{1}$ receptor to clathrin-coated pits by interacting with clathrin and the clathrin adapter 2 (AP2), which directly interact with the $\mathrm{AT}_{1}$ receptor carboxyl terminus (Fessart et al., 2005). These interactions are regulated by c-Src and the ADP-ribosylation factor 6 (ARF6), a small GTPbinding protein (Fessart et al., 2005; Poupart et al., 2007; Zimmerman et al., 2009).

After endocytosis, the receptor induces specific $\beta$-arrestin-mediated cell signaling pathways, distinct from G-protein signaling (Kim et al., 2005a). Shah et al. (2002) showed that $\beta$-arrestin-mediated ERK activation is regulated by transactivation of the EGFR and activation of GRK 5 and GRK6 (Kim et al., 2005a) but not GRK 2 and 3. The selective receptor phosphorylation on different sites by the various GRK isoforms may have important implications. For example, GRKs 5 and 6 seem to mediate effects associated with physiologic consequences, which are different from GRK 2-mediated effects (Kim et al., 2005a). Inhibition of GRK 5 or 6 attenuates $\beta$-arrestin-mediated ERK activation, whereas it is not affected by knockdown of GRK 2 or 3 (Kim et al., 2005a). An internalization-deficient mutant of the $\mathrm{AT}_{1}$ receptor with truncated carboxyl terminus can also produce these responses, implying that internalization per se is not necessary for $\beta$-arrestin-mediated signaling (Turner et al., 2001).

$\mathrm{AT}_{1}$ receptors are internalized within 10 minutes, and $25 \%$ of internalized receptors are recycled back to plasma membrane and the remainders are degraded in lysosomes (Gunther et al., 1980; Griendling et al., 1987). The Rab family of proteins, specifically Rab 1 , is associated with transport of $\mathrm{AT}_{1}$ receptor from endoplasmic reticulum to Golgi to cell surface (Wu et al., 2003). $\mathrm{AT}_{1}$ receptor traffic to early endosomes is dependent on Rab 5 interaction with the carboxyl terminus of the receptor (Daviet et al., 1999; Somsel Rodman and Wandinger-Ness, 2000; Seachrist et al., 2002). Rab 7-positive late endosomes promote AngII dissociation. Rapid receptor recycling back to the plasma membrane takes place in Rab 11-positive vesicles by a rapid PI3Kdependent pathway (Garcia-Caballero et al., 2001; Hunyady et al., 2002; Seachrist et al., 2002; Dale et al., 2004).

In addition to clathrin-dependent pathway, $\mathrm{AT}_{1}$ receptor can be internalized via specialized microdomains called caveolae, associated with caveolin (Ishizaka et al., 1998). In VSMCs, AngII regulates the expression, biosynthesis, and phosphorylation of caveolins and promotes the translocation of $\mathrm{AT}_{1}$ receptor to caveolin-enriched membrane fractions (Ishizaka et al., 1998).

\section{F. Expression and Regulation}

$\mathrm{AT}_{1}$ receptors in all organs are sensitively regulated by a number of physiologic and pathophysiological factors (Kaschina and Unger, 2003; Elton and Martin, 2007; Higuchi et al., 2007). AngII, interferon, growth factors, estrogens, statins, nitric oxide, thyroid hormone, retinoic acid, and peroxisome proliferator-activated receptor can suppress transcription of the rat $\mathrm{AT}_{1 \mathrm{a}}$ receptor gene 
in cultured rat VSMCs. In contrast, glucocorticoids, insulin-like growth factor, interleukin (IL)-6, erythropoietin, and progesterone induced an upregulation of expression in VSMCs. AngII accelerates $\mathrm{AT}_{1}$ receptor mRNA decay in VSMCs, which is governed by interaction of phosphorylated calreticulin with the $3^{\prime}$ untranslated segment 2175-2195 of the $\mathrm{AT}_{1}$ receptor mRNA (Nickenig et al., 2002). Regulation of rat $\mathrm{AT}_{1 \mathrm{a}}$ receptor promoter by cyclic AMP (Chen et al., 2002), free radicals (Nickenig et al., 2000; Chen et al., 2002), tumor necrosis factor-alpha $(\mathrm{TNF} \alpha)$, interleukin-1 beta (Cowling et al., 2002), and purinergic P2Y(Dorn and Force, 2005) receptor in cardiomyocytes (Nishida et al., 2011) are observed.

Human and rodent genes for the $\mathrm{AT}_{1}$ receptor are thought to be regulated by different mechanisms, because the promoter regions are divergent. Expression of the $\mathrm{hAT}_{1}$ receptor is regulated predominantly by Sp1 and Sp3. MEF-2 and Sp1 regulate basal expression of the rat $\mathrm{AT}_{1 \mathrm{a}}$ receptor gene. PPAR suppresses rat $\mathrm{AT}_{1 \mathrm{a}}$ receptor gene the transcription by inhibiting Sp1 binding. Thus PPAR ligands may inhibit AngII-induced cell growth and hypertrophy in VSMCs by inhibiting $\mathrm{hAT}_{1}$ receptor expression. In contrast, PPAR and CBP enhanced activity of $\mathrm{Sp} 1$ increase transcription of rat $\mathrm{AT}_{1 \mathrm{a}}$ receptor gene. Oxidized low-density lipoprotein (LDL) upregulates $\mathrm{AT}_{1}$ receptor expression in cultured human coronary artery endothelial cells (Li et al., 2000).

\section{G. Pathophysiological Aspects of AngII Type 1 Receptor Activation}

AngII signaling through the $\mathrm{AT}_{1}$ receptor promotes pathogenic processes such as ROS production, inflammation, altered vasoreactivity, growth, migration, platelet activation, and fibrosis. Ultimately these cause diseases such as hypertension, atherosclerosis, thrombosis, chronic kidney disease, and insulin resistance, with the final development of cardiovascular disease. Improved clinical outcomes after treatment with ARBs proves the causative role of $\mathrm{AT}_{1}$ receptor in the pathogenesis of these diseases (Garg and Yusuf, 1995; Yusuf et al., 2000; Igarashi et al., 2001).

1. Cardiovascular Remodeling and Hypertrophy. In vitro and in vivo experiments have shown growth promoting actions of AngII, causing cardiac and vascular hypertrophy, cell differentiation, and apoptosis (Pfeffer and Braunwald, 1990; Lombardi et al., 1999; Lips et al., 2003; Dorn and Force, 2005). In general, ARBs effectively prevent cardiac, vascular, and renal hypertrophy (Kim et al., 1995, 1998). Involvement of ERK1/2, PI3K, and CDK2 inhibition pathways, leading to G1-phase arrest, causes myocyte hypertrophy (Braun-Dullaeus et al., 1999). Increase in protein synthesis involves activation of translation elongation factor-2 in cardiac myocytes via dephosphorylation by PP2A by a process that involves both PI3K and MAPK (Everett et al., 2001). Increased protein synthesis through $\mathrm{AT}_{1}$ receptors in human cardiac fibroblasts did not induce hypertrophy of cardiac fibroblast (Hou et al., 2000). In the vasculature, DNA synthesis is enhanced upon AngII infusion through the activation of cyclin D1 and cdk4 and reduction in the expression of cell cycle kinase inhibitors p21 and p27 (Diep et al., 2001; Guillemot et al., 2000, 2001). Thus, in the myocardium, regulation of growth effects by $\mathrm{AT}_{1}$ receptor in myocytes and fibroblasts differs. Cardiac hypertrophy includes cardiac myocyte enlargement and proliferation of cardiac fibroblasts. Now it is generally believed that both hypertrophic response of myocytes and proliferative response of fibroblast may depend on other modifying factors such as production of ROS and secretion of various types of factors.

For instance, in pressure overload due to hypertension and myocardial infarction, cardiac remodeling process includes cardiomyocyte hypertrophy, extracellular matrix synthesis, fibrosis, and loss of compliance, leading to fatal outcomes. TGF- $\beta 1$ expression is increased in myocytes and fibroblasts of heart, which transdifferentiate into a myofibroblast phenotype, resulting in myocardial remodeling (Campbell and Katwa, 1997). The $\mathrm{AT}_{1}$ receptor directly increases TGF- $\beta 1$ expression (Kupfahl et al., 2000; Schultz et al., 2002), translocation of Smad proteins Smad 2 and 4 into the nucleus, resulting in expression of fibrotic marker proteins, collagen, fibronectin, and connective tissue growth factor (CTGF) (Hao et al., 2000; Rodriguez-Vita et al., 2005; Lim and Zhu, 2006; Zhang et al., 2009). CTGF is a profibrotic factor that stimulates both AngII- and TGF- $\beta$-1-mediated fibrosis and apoptosis (Abreu et al., 2002; Perbal, 2004; Cabello-Verrugio et al., 2011). CTGF is involved in myocardial remodeling mediated via $\mathrm{AT}_{1}$ receptors during transition to HF (Iwanciw et al., 2003; Ahmed et al., 2004). AngII-induced CTGF production is also shown in the aorta of AngII-infused rats (Ruperez et al., 2003). AngII-stimulated collagen synthesis in aortic adventitial fibroblasts, which is actively involved in vascular remodeling, is mediated by CTGF (Che et al., 2008). Gene expression analysis detected high CTGF mRNA expression in coronary artery biopsies from ischemic injury and coronary artery disease. Proteins involved in extracellular matrix remodeling, such as thrombospondin 4, collagen type 1 and 2 , and fibronectin, and the inflammatory cytokines, such as IL-8, IL-6, vascular cell adhesion molecule-1, and monocyte chemoattractant protein-1 (MCP-1), are believed to couple cardiac remodeling with chronic angiotensin receptor stimulation (Gabrielsen et al., 2007). IL-6 secretion by cardiac myocytes is regulated by AngII. The effects of IL-6 on cardiomyocyte hypertrophy and fibroblast proliferation is inhibited by the $\mathrm{AT}_{1}$ receptor antagonist losartan, suggesting that IL- 6 contribution to cardiomyocyte hypertrophy is mediated by the $\mathrm{AT}_{1}$ receptor (Fredj et al., 2005). AngII-induced activation of the JAK/STAT pathway is involved in tissue remodeling 
after vascular injury and myocardial ischemia in rats (Seki et al., 2000; Omura et al., 2001). The $\mathrm{AT}_{1}$ receptor activates STAT1 and GATA4 transcription factors in the development of myocyte hypertrophy (Wang et al., 2005). Chronic activation of the $\mathrm{AT}_{1}$ receptor in myocytes induces transcription of the Stat3 gene by pSTAT3 and overproduction of STAT3 protein, leading to nuclear accumulation of STAT3 without tyrosine phosphorylation, which alters the transcriptional program of cardiac hypertrophy (Yue et al., 2010).

VSMC hypertrophy induced by AngII involves PKC delta activation through Src-dependent Tyr phosphorylation, leading to Akt activation and signifying a novel molecular mechanism for enhancement of cardiovascular diseases induced by AngII (Nakashima et al., 2008). Antiapoptotic effects of AngII in cardiomyocytes and VSMCs are regulated by a mechanism involving PI3-kinase/Akt activation, subsequent upregulation of survivin, and suppression of caspase-3 activity (Ohashi et al., 2004). Inhibitors of Akt and a dominant-negative mutant of Akt selectively block AngII-induced proliferation of CHO-AT $1 \mathrm{a}$ cells (Dugourd et al., 2003). AngII activated reactive oxygen species acting through Src/ caveolin-EGFR signaling pathway induces epithelialto-mesenchymal transition in renal epithelial cells. This may be a novel molecular mechanism involved in progressive renal injury caused by chronic exposure to AngII (Chen et al., 2012). Adenoviral-directed expression of the $\mathrm{AT}_{1}$ receptor has defined the EGFR transactivation pathway for cardiac hypertrophy via PI3K/Akt signaling (Ebert et al., 1995; Thomas et al., 2002).

2. Vascular Inflammation and Atherosclerosis. The role of AngII in atherosclerosis has been well established. Atherosclerotic risk factors such as hypercholesterolemia and hypertension also increase production of angiotensinogen (Daugherty et al., 2004). Inhibition of $\mathrm{AT}_{1}$ receptor $\mathrm{s}$ by losartan prevents lipid peroxidation, decreasing atherosclerotic lesion formation $\mathrm{n}$ apolipoprotein E-deficient mice (Keidar et al., 1997). Conversely, AngII infusion increases aortic atherosclerosis and aneurysm formation, independent of blood pressure (Daugherty et al., 2000; Weiss et al., 2001; AbdAlla et al., 2001a). Male apoE/AT $1 \mathrm{a}$ receptor double knockout mice have reduced atherosclerosis (Wassmann et al., 2004a). This relationship between $\mathrm{AT}_{1}$ receptor and atherosclerosis is conserved in hypercholesterolemic rabbits (Yang et al., 1998). Stimulation of inflammatory mediators including IL- $1 \beta$, IL-6, and $\mathrm{TNF} \alpha$ through $\mathrm{NF}_{\kappa} \mathrm{B}$ activation (Sanz-Rosa et al., 2005) and RAS activation appears to be the pathogenic mechanism in the atherosclerotic process. AngII and/or IL-6 infusion induces oxidative stress and endothelial dysfunction in mice and these effects are completely abolished in $\mathrm{AT}_{1}$ receptor knockout mice (Schieffer et al., 2000; Ruiz-Ortega et al., 2001b; Wolf et al., 2002; Skurk et al., 2004; Wassmann et al., 2004b). In VSMCs, the activation of $\mathrm{NAD}(\mathrm{P}) \mathrm{H}$ oxidase by the $\mathrm{AT}_{1}$ receptor produces IL-6
(Marui et al., 1993; Chen et al., 1998; Kranzhofer et al., 1999) and $\mathrm{AT}_{1}$ receptor blockers decreases $\mathrm{TNF} \alpha$, IL-6, and soluble adhesion molecules (Tsutamoto et al., 2000). Secretion of inflammatory factors, such as P-selectin and MCP-1 that are involved in the vascular inflammation and atherogenesis is $\mathrm{AT}_{1}$ receptor-dependent, and $\mathrm{ARBs}$ attenuate P-selectin and MCP-1 expression with concurrent reduction in intimal proliferation in mice (Chen et al., 2001). In hepatocytes, CARMA3/Bcl10/MALT1dependent $\mathrm{NF}_{\kappa} \mathrm{B}$ activation mediates AngII-responsive inflammatory signaling promoting pathologic liver fibrosis (McAllister-Lucas et al., 2007). CARMA1 and 3 are expressed in VSMC and endothelial cells, suggesting the potential for pathway similar to hepatocytes playing a role in vascular inflammation and atherosclerosis. The $\mathrm{AT}_{1}$ receptor stimulates myofibroblasts to proliferate through activation of the $\mathrm{NF}_{\kappa} \mathrm{B}$ transcription factor via a signaling pathway composed of CARMA3, Bcl10, and MALT1 (McAllister-Lucas et al., 2007).

Under chronic liver injury, AngII promotes pathologic liver fibrosis by stimulating hepatocytes and hepatic stellate cells to synthesize extracellular matrix proteins and secrete secondary cytokines. In rats, experimental models of liver fibrosis induced by bile duct ligation or carbon tetrachloride or choline deficiency, administration of irbesartan, olmesartan, telmisartan, candesartan, or losartan inhibited expression of collagens and TGF $\beta$ in stellate cells and reduced established liver fibrosis (Kurikawa et al., 2003; Ueki et al., 2006; Hirose et al., 2007; Yoshiji et al., 2009; Moreno et al., 2010; Kato et al., 2012). In clinical practice, however, the usefulness of treating liver fibrosis with ARBs remains contradictory (Schneider et al., 1999; Gonzalez-Abraldes et al., 2001; Lee, 2014). A handful of randomized controlled trials suggest that ARB treatment is a potentially useful therapeutic approach in patients with nonalcoholic fatty liver disease (Paschos and Tziomalos, 2012). The nonalcoholic fatty liver disease patients benefited from telmisartan and olmesartan treatment (Enjoji et al., 2008; Colmenero et al., 2009). In two independent pilot studies, administration of candesartan or losartan was shown to have an antifibrotic effect on patients with chronic hepatitis C (Sookoian et al., 2005; Ueki et al., 2009).

3. Endothelial Dysfunction. Endothelial dysfunction refers to impairment of endothelium-dependent vasodilation associated with progressive changes in cell adhesion and barrier function. Inactivation of NO by $\mathrm{AT}_{1}$ receptor-induced ROS is at the center of endothelial dysfunction in hypertension, atherosclerosis, and cardiovascular diseases. In endothelial cells, eNOS produces NO and a low amount of superoxide to maintain vasodilation and a healthy vasculature. In disease state, redox-uncoupled eNOS is responsible for disproportionate production of superoxide relative to NO. The excessive superoxide reacts with $\mathrm{NO}$ to form peroxynitrite, a toxic 
radical, which directly contributes to disease state. Endothelial cells in a disease state express adhesion molecules, which increase adhesion of circulating inflammatory blood cells into the vessel wall, leading to further increase in ROS production (Rajagopalan et al., 1996; Schena et al., 1999; Zhao et al., 2005a). ROS increases local RAS activation in the vasculature, enhancing AngII production in ECs, VSMCs, and fibroblasts and creates a mutual reinforcement loop between local RAS and ROS. The $\mathrm{AT}_{1}$ receptor induces LDL receptor expression in ECs (Gryglewski et al., 1986; Rubanyi and Vanhoutte, 1986; Li and Mehta, 2000) and enhances the modification of LDL and the expression of its lectin-like receptor (LOX-1), which are critical events in atherosclerotic lesion formation (Chen et al., 2000a; Figueroa and Vijayagopal, 2002). Overall endothelial dysfunction is characterized by blunted endotheliumdependent vasodilation associated with enhanced contraction in hypertensive diseases (Dasgupta and Zhang, 2011).

4. Oxidative Stress. $\mathrm{AT}_{1}$ receptor-regulated oxidative stress plays a major part in the initiation and progression of hyperlipidemia, diabetes mellitus, hypertension, ischemic heart disease, and chronic HF (Marui et al., 1993; Taniyama and Griendling, 2003). ROS activates nuclear factor $\kappa \mathrm{B}(\mathrm{NF} \kappa \mathrm{B})$ and stimulates degradation of its cytoplasmic inhibitor, $\mathrm{I} \kappa \mathrm{B}$, an essential step in the proinflammatory process (Pueyo et al., 2000). Blockade of $\mathrm{NF}_{\kappa} \mathrm{B}$ ameliorates myocardial hypertrophy in response to infusion of AngII, and the effect of AngII was attenuated in mice with targeted disruption of the p50 subunit of $\mathrm{NF}_{\kappa} \mathrm{B}$ (Kawano et al., 2005). $\mathrm{NF}_{\kappa} \mathrm{B}$ gene expression results in increased levels of vascular cell adhesion molecule- 1 and probably other genes involved in the early stages of atherosclerosis (Pueyo et al., 2000). AngII/AT 1 receptor/ $\mathrm{NF}_{\kappa} \mathrm{B}$ pathway may be involved in brain ischemia by stimulating intercellular adhesion molecule-1 expression in brain microvascular ECs (Liu et al., 2006). Thus, $\mathrm{AT}_{1}$ receptorinduced ROS production can change structure-function properties of the vasculature that is the central aspect of vascular pathology in hypertension and diabetes. Treatment with ARBs stimulates NO release in platelets and ECs indicating arterial antithrombotic effects of ARBs (Kalinowski et al., 2002).

5. Extracellular Matrix Deposition. AngII is a risk factor implicated in cardiac remodeling, and ARB treatment is protective (Nagata et al., 2002). $\mathrm{AT}_{1}$ receptor signaling is increasingly recognized for its profibrotic effects in other tissues as well. Mechanism of fibrosis may differ in different tissues. In the heart, the $\mathrm{AT}_{1}$ receptor upregulates TGF- $\beta 1$, laminin, and fibronectin expression and contributes to increased cardiac fibroblast attachment to collagens I and III and increased focal adhesion kinase activity. Synthesis of the extracellular matrix proteins upon $\mathrm{AT}_{1}$ receptor activation (Kato et al., 1991; Mifune et al., 2000) involves transactivation of EGFR-MAPK-dependent pathways (Ju and Dixon, 1996; Touyz et al., 2001a). Abnormal accumulation of proteoglycans is known in atherosclerotic lesions (Evanko et al., 1998; Iozzo, 1998), and treatment with ARBs induces proteoglycan changes that favor healthier cell adhesion, migration, and differentiation (Iozzo, 1998; Moriguchi et al., 1999; Sasamura et al., 2001). In aortic VSMCs and ECs as well as in cardiac cells, the $\mathrm{AT}_{1}$ receptor-EGFR transactivation pathway also regulates fibronectin synthesis, production of matrix metalloproteinases and breakdown of collagen IV, expression of plasminogen activator inhibitor-1 (Feener et al., 1995; Chen et al., 2000b; Kawano et al., 2000; Nakamura et al., 2000). Regulation of PAI-1 by the $\mathrm{AT}_{1}$ receptor seems to be important in many contexts; for instance, in human adipocytes, impairment of the fibrinolysis has been implicated in obesity (Skurk et al., 2001) and in reduced trophoblast invasion (Xia et al., 2002; Abbasi et al., 2005). $\mathrm{AT}_{1}$ receptor blockade effectively reduces AngII-stimulated PAI-1 secretion (Sironi et al., 2001). In skin wound healing, $\mathrm{AT}_{1}$ receptorstimulated keratinocyte and fibroblast migration mediated by EGFR transactivation (Yahata et al., 2006) causes an increase in TGF- $\beta 1$ and integrin protein levels (Thibault et al., 2001). This wound-healing pathway is attenuated in $\mathrm{AT}_{1}$ receptor-knockout mice. Thus, the $\mathrm{AT}_{1}$ receptor regulates formation of ECM components and turnover of matrix. The mechanisms and pathways that integrate ECM formation and turnover in relation to AngII signaling are still being discovered.

6. Insulin Resistance. Patients with an imbalance in RAS homeostasis exhibit decreased insulin sensitivity (Nickenig et al., 1997; Kurtz and Pravenec, 2004), and treatment with ARBs improves insulin resistance and diabetic complications (Henriksen et al., 2001; Igarashi et al., 2001; Kurtz and Pravenec, 2004). Studies in rats show that $\mathrm{AT}_{1}$ receptor activation hinders insulin signaling upon infusion of AngII to cause insulin resistance (Patiag et al., 2000; Ogihara et al., 2002). Normally, insulin binding to insulin receptor enhances its tyrosine kinase activity and tyrosine phosphorylation of the insulin receptor substrates (IRS) and activation of phosphatidylinositol-3 kinase (PI3K). In rat VSMCs, AngII impairs coupling of the insulin receptor (IGF-1R) to PI3K and inhibits insulin-mediated IRS-1 tyrosine phosphorylation and association of IRS-1 with p85 (Folli et al., 1997). Alternatively, AngII increases serine phosphorylation of IRS-1 (Ser616 via ERK and Ser312 via JNK), thus interfering with insulin signaling (Andreozzi et al., 2004). Another mechanism described for interference with insulin signaling involves AngII-dependent tyrosine phosphorylation of PDK1 and ROS-sensitive Src activation (Taniyama et al., 2004; Taniyama et al., 2005). PKC is another kinase that may interfere with insulin signaling (Motley et al., 2003). Thus, $\mathrm{AT}_{1}$ receptor signals may hamper insulin action at multiple levels. Hypertension and diabetes are frequently seen together, 
indicating that interaction between AngII and insulin signaling plays an important role in cardiovascular pathology. ACE inhibitors are effective in the treatment of neuropathy found in diabetes (Malik et al., 1998; Malik, 2000). Coppey et al. (2006) showed that L-158809 (2-ethyl-5,7-dimethyl-3-[[4-[2-(2H-tetrazol-5-yl) phenyl]phenyl]methyl]imidazo[5,4-b]pyridine), an AngII receptor blocker, attenuated diabetic neuropathy in streptozotocin-induced diabetic rats. Until now, there are no clinical studies on the effect of ARBs in diabetic neuropathy, although the results suggest that ARB application may bear promise for treating neuropathy associated with vascular dysfunction and diabetic condition.

7. Angiogenesis and Cancer. $\mathrm{AT}_{1}$ receptor-regulated cell proliferation and angiogenesis has biologic and therapeutic implications in cancer (Escobar et al., 2004; Deshayes and Nahmias, 2005; Uemura et al., 2005, 2006, 2011; Ino et al., 2011; Lau and Leung, 2011; Uemura and Kubota, 2012). Local AngII production is a proangiogenic stimulus in tumor microenvironment. Tumors implanted in wild-type mice developed intensive angiogenesis with vascular endothelial growth factor (VEGF) induction in tumor stroma. Systemic administration of an ARB reduced tumor-associated angiogenesis and VEGF expression in tumor stroma. In comparison, tumor-associated angiogenesis was reduced in $\mathrm{AT}_{1 \mathrm{a}} \mathrm{R}$ null mice, which was characterized by reduced expression of VEGF in the stroma and also reduced infiltration by macrophages. AngII-induced angiogenic factors production involves $\mathrm{AT}_{1}$ receptor/ JAK2/STAT3/SOCS3 signaling pathway. These results suggest that host stromal VEGF induction by $\mathrm{AT}_{1}$ receptor is a key regulator of tumor growth and blockade of VEGF production by ARBs may be a novel therapeutic strategy against cancers (Egami et al., 2003; Fujita et al., 2005). AngII has been shown to function as a key role in neovascularization of hepatocellular carcinoma (Tamarat et al., 2002) in human breast carcinoma cells (Greco et al., 2002) as well as in invasive ductal breast cancer (Jethon et al., 2012). Both lisinopril and losartan treatment resulted in elevation in VEGF expression and angiogenesis, confirming the relationship between $\mathrm{AT}_{1}$ receptor, VEGF, and vessel growth (Tamarat et al., 2002).

The choriocarcinoma cell proliferation is enhanced by AngII through the $\mathrm{AT}_{1}$ receptor and activation of protein kinase $\mathrm{C}-$ and mitogen-activated protein kinase (Ino et al., 2003). AngII plays a role in the growth and chemoresistance of $\mathrm{AT}_{1}$ receptor-positive pancreatic cancer cells through its action as a potent mitogen and antiapoptotic molecule (Amaya et al., 2004). AngII may promote prostate tumorigenesis via upregulation of PAX2 expression (Bose et al., 2009; Zhao et al., 2010). Thus, in the context of cancers of multiple tissues,

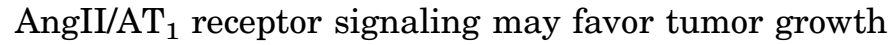
and may also contribute to aggressive etiology of cancers. Consideration of ARBs to restrict tumor growth should be a front line antitumor growth approach.

8. Autoantibodies and Malignant Hypertension. Autoantibodies that bind to and activate the $\mathrm{AT}_{1}$ receptor exist in patients with hypertensive disorders and contribute to disease pathophysiology in preeclampsia, in kidney transplant recipients who develop refractory vascular rejection, and in patients with malignant hypertension (Roberts, 2000; Lodwick, 2001; Dechend et al., 2004; Herse et al., 2008; LaMarca et al., 2011; Herse and LaMarca, 2013; Xia and Kellems, 2013). More recently, $\mathrm{AT}_{1}$ receptor-directed autoantibodies have been seen in patients with the autoimmune diseases, including systemic sclerosis, featuring autoimmunity, vasculopathy, and tissue fibrosis (Fu et al., 2000; Liao et al., 2002; Ansari et al., 2005; Dragun et al., 2005; Riemekasten et al., 2011). The $\mathrm{AT}_{1}$ receptor autoantibodies found in preeclampsia, renal allograft rejection, and malignant hypertension are directed to an epitope, -AFHYESQ-, in the second extracellular loop of the $\mathrm{AT}_{1}$ receptor (Wallukat et al., 1999). Treatment with $\mathrm{AT}_{1}$ receptor blocker reverses the pathophysiological effects of $\mathrm{AT}_{1}$ receptor autoantibodies in these diseases, suggesting the ability of autoantibodies to activate $\mathrm{AT}_{1}$ receptors (Dechend et al., 2000; Dorffel et al., 2003). Autoantibody-induced production of reactive oxygen species (ROS) by the placenta and maternal tissues likely contributes to the oxidative stress associated with preeclampsia (Hubel, 1999; Many et al., 2000; Sikkema et al., 2001; Dechend et al., 2003; Thway et al., 2004). Injection of pregnant mice with IgG from preeclamptic patients leads to hypertension, proteinuria, and preeclampsia in mice (Zhou et al., 2008b). These features were prevented by an antibody-neutralizing, sevenamino-acid epitope peptide (Zhou et al., 2007, 2008a). The presence of $\mathrm{AT}_{1}$ receptor autoantibodies was also confirmed in the experimental rat models of preeclampsia as extensively reviewed by Xia and Kellems (2013) recently.

\section{The Angiotensin II Type 2 Receptor}

Despite the controversial role it plays, the literature on the $\mathrm{AT}_{2}$ receptor is quite extensive and confusing as reflected by 1061 peer-reviewed articles mined in our search (Fig. 1; Kemp, Karnik et al., 2014, http://www. guidetopharmacology.org/GRAC/ObjectDisplayForward? objectId=35.). The $\mathrm{AT}_{2}$ receptor is a seven-transmembrane helical receptor that shares approximately $34 \%$ amino acid sequence homology with the $\mathrm{AT}_{1}$ receptor. Its discovery in the 1980s as the dithiothreitol-potentiated or the PD123319-binding AngII receptor in vivo, cDNA cloning, identification of the gene, and generation of geneknockout and transgenic mice in 1990s highlight the history of the $\mathrm{AT}_{2}$ receptor (de Gasparo et al., 2000). The AGTR2 gene is localized in the human chromosome Xq22q23. Gene Agtr2 in the rat is located on chromosome Xq34 
and in mouse on chromosome at X12.5 cM. Gene organization is conserved in human and rodents, consisting of three exons, two introns, and the entire proteincoding frame contained in the third exon (de Gasparo et al., 2000). The first two exons contain a $5^{\prime}$-untranslated region (Vervoort et al., 2002). The $\mathrm{AT}_{2}$ receptor is evidently different from the $\mathrm{AT}_{1}$ receptor in terms of gene, genetic variations, protein sequence, tissuespecific expression, signaling mechanisms, regulation of receptor function, and pharmacological property. The in vivo physiologic functions of the $\mathrm{AT}_{2}$ receptor are still not clearly defined. Fifteen years of research and $>2500$ publications devoted to $\mathrm{AT}_{2}$ receptor delineate the discovery of unconventional ligand selectivity, agonistindependent signaling, and cGMP/NO signaling by $\mathrm{AT}_{2}$ receptor. Identification of novel $\mathrm{AT}_{2}$ receptor-interacting proteins and pharmacological agonists in recent years rekindled interest in this enigmatic receptor that could be therapeutically exploited for a possible protective role. This aspect of the $\mathrm{AT}_{2}$ receptor is focused on in this review.

\section{A. Structure}

The human, rat, and mouse $\mathrm{AT}_{2}$ receptor cDNAs encode a 363-amino acid protein that harbors hallmarks of a typical GPCR (Nakajima et al., 1993; Kambayashi et al., 1994; Koike et al., 1994). The $\mathrm{AT}_{2}$ receptor amino acid residues show $72 \%$ divergence between rodents and humans, whereas the sequence is $99 \%$ conserved between rat and mouse. The $\mathrm{AT}_{2}$ receptor encodes five potential $N$-glycosylation sites, which account for diverse molecular weights $(68-113 \mathrm{kDa})$ observed in different tissues. Two potential disulfide bonds located in the $\mathrm{AT}_{2}$ receptor extracellular region account for its characteristic DTT potentiation, which distinguishes it from the $\mathrm{AT}_{1}$ receptor (Speth et al., 1991; Feng et al., 2000). The amino acid sequence identity between regions of the $\mathrm{AT}_{2}$ receptor and $\mathrm{AT}_{1}$ receptor varies between 24 to $34 \%$ in the transmembrane domain. The sequence of the third intracellular loop and the carboxyl terminal tail in $\mathrm{AT}_{2}$ receptor diverge substantially from $\mathrm{AT}_{1}$ receptor. These structural features of the $\mathrm{AT}_{2}$ receptor form a potential basis for its poor coupling to G-proteins and lack of phosphorylation by GRKs as well as lack of desensitization after AngII binding. Although it was cloned over 15 years ago, little progress has been made with regard to solving the three-dimensional structure and identifying ligandbinding residues of the $\mathrm{AT}_{2}$ receptor. The mechanism of ligand recognition and transmembrane signaling by the $\mathrm{AT}_{2}$ receptor remains unexplored, which should be a priority in light of the possible protective role of the $\mathrm{AT}_{2}$ receptor.

\section{B. Pharmacology}

Natural peptide hormone ligands AngII and AngIII bind the $\mathrm{AT}_{2}$ receptor with nanomolar affinity and do not distinguish it from the $\mathrm{AT}_{1}$ receptor. Although the semipeptide CGP42112 is an agonist for the $\mathrm{AT}_{2}$ receptor, it is a nonspecific agonist for $\mathrm{AT}_{1}$ receptor at high concentration $\left(K_{\mathrm{i}} 1.7 \mu \mathrm{M}\right)$ (Brechler et al., 1993; Macari et al., 1994; de Gasparo et al., 2000). The analog, [p-amino-Phe $\left.{ }^{6}\right]$ AngII is a classic ligand used to discriminate between $\mathrm{AT}_{1}$ and $\mathrm{AT}_{2}$ receptors. An additional potential endogenous agonist of the $\mathrm{AT}_{2}$ receptor is vasoconstriction-inhibiting factor (VIF), which was isolated from human adrenal glands. VIF is a vasoregulatory peptide that modulates the vasoconstrictive effects of AngII by acting on the $\mathrm{AT}_{2}$ receptor (Salem et al., 2015). VIF has been shown to inhibit AngII-induced phosphorylation of the p38 mitogen-activated protein kinase pathway but not of extracellular-regulated kinase 1/2 (Salem et al., 2015). A previously held view that AngI is a natural ligand for the $\mathrm{AT}_{2}$ receptor is incorrect. Despite recognizing the same physiologic ligand, the pharmacophore for the $\mathrm{AT}_{2}$ receptor is distinct from that of the $\mathrm{AT}_{1}$ receptor (Miura and Karnik, 1999). Molecular recognition of AngII by the $\mathrm{AT}_{2}$ receptor is "relaxed," operating upon a Lilliputian principle in that side-chain modifications of AngII that are detrimental to $\mathrm{AT}_{1}$ receptor binding affinity are well tolerated by the $\mathrm{AT}_{2}$ receptor. The discovery that interaction of no individual residue in AngII is critical for affinity of binding explains the ability of this receptor to engage analogs and metabolites of AngII (Miura and Karnik, 1999).

The nonpeptide antagonists PD123319 (ditrifluoroacetate) and PD123177 (trifluoroacetate salt) are widely used tools. PD123319 has a high affinity for the $\mathrm{AT}_{2}$ receptor $\left(K_{\mathrm{i}} \sim 12 \mathrm{nM}\right)$ and is approximately 10,000-fold more selective for $\mathrm{AT}_{2}$ than $\mathrm{AT}_{1}$ receptors. $\mathrm{AT}_{2}$ receptor selectivity has been an important tool in defining the pharmacology and functions of this receptor in several different types of cells and tissues (Chiu et al., 1989; Chang and Lotti, 1990; Dudley et al., 1990; Wiest et al., 1991; Dudley and Summerfelt, 1993). Experimentally, the $\mathrm{AT}_{2}$ receptor has been observed to bind a variety of ligands with an affinity order, CGP42112 $>$ AngII $\geq$ AngIII $>$ Compound $21 \geq$ PD123319 $>>$ AngIV $>$ Ang (1-7) in the $\mathrm{AT}_{2}$ receptor-transfected HEK-293 cells (Jones et al., 2011; Sipahi et al., 2011). Shorter angiotensin peptides may act as endogenous ligands at the $\mathrm{AT}_{2}$ receptor; therefore, defining the physiology as well as the concept of separate receptors for AngII-metabolite peptides should formally rule out $\mathrm{AT}_{2}$ receptor-mediated effects.

Research on the $\mathrm{AT}_{2}$ receptor, specifically defining the beneficial effect, has long been hampered because of its low expression level in the adult and lack of ligands with pharmacological specificity. However, an $\mathrm{AT}_{2}$ receptor-selective nonpeptide agonist, Compound 21 (Wan et al., 2004), and its application for defining in vivo function of this receptor is attracting attention to expound the distinct roles of the $\mathrm{AT}_{2}$ receptor in many physiologic and pathophysiological states. 
The $\mathrm{AT}_{2}$ receptor is a new target for development of novel therapeutic agents to treat neuropathic pain. Human sensory neurons selectively express $\mathrm{AT}_{2}$ receptor and not $\mathrm{AT}_{1}$ receptor and hence may play a role in nociception (Anand et al., 2013; Smith et al., 2013a,b). In general, treatments for neuralgia and neuropathic pain are limited by poor efficacy and unfavorable side effects. The $\mathrm{AT}_{2}$ receptor antagonist PD123319 and some analogs have been developed into orally active drugs for neuropathic pain (Smith et al., 2013a,b). EMA401 ((S)-2-(diphenylacetyl)-1,2,3,4-tetrahydro-6-methoxy-5(phenylmethoxy)-3-isoquinolinecarboxylic acid), a highly selective $\mathrm{AT}_{2}$ receptor antagonist, has been shown to inhibit capsaicin-evoked calcium influx in human and rodent sensory neuron cultures (Anand et al., 2013). In a phase-two clinical trial, EMA401 was found to provide superior relief of postherpetic neuralgia compared with placebo and was well tolerated by patients (Rice et al., 2014). EMA300 (5-[2,2-di(phenyl)acetyl]-4-[(4methoxy-3-methylphenyl)methyl]-1,4,6,7-tetrahydroimidazo [4,5-c]pyridine-6-carboxylic acid), another small molecule antagonist of the $\mathrm{AT}_{2}$ receptor, was found to alleviate neuropathic pain in mice with a chronic constriction injury of the sciatic nerve. Smith et al. (2013a,b) also showed that augmented Ang $\mathrm{II} / \mathrm{AT}_{2}$ receptor signaling in the dorsal root ganglia of chronic constriction injury rats was attenuated by EMA300, blocking p38 MAPK and p44/p42 MAPK activation and producing analgesia. Recently, EMA200 and EMA300 were assessed in a rat model of dideoxyxytidine-induced antiretroviral toxic neuropathy (ATN) (Smith et al., 2014). ATN is commonly observed in individuals infected with HIV and taking certain antiretroviral drugs to suppress viral replication. These individuals have a high prevalence of neuropathic pain and, therefore, a great need for new analgesics with $\geq 1000$-fold selectivity over the $\mathrm{AT}_{1}$ receptor. Administration of EMA200 and EMA300 induced dose-dependent analgesia in dideoxyxytidine rats, suggesting that these $\mathrm{AT}_{2}$ receptor-specific analgesics should be investigated further for the relief of ATN (Smith et al., 2014). Further assessment of these and additional small molecule antagonists will advance the field of neuralgia management to develop promising therapeutics aimed at targeting the $\mathrm{AT}_{2}$ receptor.

Based on the relaxed "conformation" hypothesis, $\mathrm{AT}_{2}$ receptor was predicted to harbor high constitutive activity (Miura and Karnik, 1999). Several lines of evidence have indeed confirmed that $\mathrm{AT}_{2}$ receptor functions in the absence of its ligand. The $\mathrm{AT}_{2}$ receptor induces apoptosis in the absence of AngII stimulation, and this effect is not modulated by PD123319 (Miura and Karnik, 2000). Similarly, in neonatal cardiomyocytes, adenoviralmediated $\mathrm{AT}_{2}$ receptor expression induces myocyte growth, which is an effect not modulated by AngII, PD123319, or CGP42112 (nicotinic acid-Tyr- $N$-benzoxyl-carbonylArg-Lys-His-Pro-Ile-OH) (D'Amore et al., 2005). In human coronary artery endothelial cells, lentiviral delivery of the $\mathrm{AT}_{2}$ receptor changes expression of a large number of genes without $\mathrm{AT}_{2}$ receptor ligands, and many fewer genes were differentially expressed when the $\mathrm{AT}_{2}$ receptorspecific ligand CGP42112 was added (Falcon et al., 2005). Kemp et al. (2014b) found that $\mathrm{AT}_{2}$ receptor expression antagonized regulation of microRNAs by $\mathrm{AT}_{1}$ receptor and AngII stimulation of $\mathrm{AT}_{2}$ receptor affected expression of only a few microRNAs, whereas the same treatment caused a robust response from $\mathrm{AT}_{1}$ receptor. These findings suggest that altered expression of $\mathrm{AT}_{2}$ receptor itself is a stimulus for function and that many cellular effects of $\mathrm{AT}_{2}$ receptor expression are not contingent on ligand interaction with this receptor.

\section{Mouse Models}

Mouse models of $\mathrm{AT}_{2}$ receptor deletion and overexpression have been useful tools for researchers to dissect the role of this receptor in cardiovascular and renal disease states. $\mathrm{AT}_{2}$ receptor null mice have increased blood pressure compared with wild-type animals along with a host of other consequences, including increased sensitivity to injected AngII, attenuation of exploratory behavior, delay in VSMC differentiation, and increased susceptibility to renal-tubular developmental disease (Hein et al., 1995; Ichiki et al., 1995; Ichihara et al., 2001).

There are no gross developmental abnormalities in $\mathrm{AT}_{2}$ receptor null mice, but blood pressure was found to be either unchanged (Hein et al., 1995) or increased (Ichiki et al., 1995) in these $\mathrm{AT}_{2}$ receptor-null mice. Based on blood pressure elevation and augmented vascular sensitivity to AngII observed in the $\mathrm{AT}_{2}$ receptor-null mice, $\mathrm{AT}_{2}$ receptor action in vasculature has been suggested to be protective, counteracting blood pressure regulation by the $\mathrm{AT}_{1}$ receptor (Hein et al., 1995; Ichiki et al., 1995). Several mechanisms may contribute to the protective effect, including regulation of $\mathrm{AT}_{1}$ receptor expression (Tanaka et al., 1999), vascular bradykinin, and cGMP/NO production (Padia and Carey, 2013). The changes in exploratory behavior and greater stimulation of dipsogenesis in the $\mathrm{AT}_{2}$ receptor null mice suggest neurologic dysfunction (Hein et al., 1995; Ichiki et al., 1995). The $\mathrm{AT}_{2}$ receptor is prominently expressed in distinct brain areas such as the locus coeruleus (Rowe et al., 1990) and the amygdaloid nucleus (Song et al., 2002). Using riboprobe in situ hybridization histochemistry, Lenkei et al. (1997) mapped the distribution of $\mathrm{AT}_{2}$ receptor mRNAs in the adult rat and found a predominant expression in the brain regions of the subfornical organ, the hypothalamus, and the lateral septum. They reported very limited overlap between the brain expression of $\mathrm{AT}_{1 \mathrm{~A}}$ and $\mathrm{AT}_{2}$ receptor mRNAs. de Kloet et al. (2014) used bacterial artificial chromosome transgenic $\mathrm{AT}_{2}$ receptor-enhanced green fluorescent protein (eGFP) reporter mouse to localize $\mathrm{AT}_{2}$ receptors at a cellular level. The authors examined for colocalized eGFP and $\mathrm{AT}_{2}$ receptor mRNA within the 
brain. This mapping approach localized $\mathrm{AT}_{2}$ receptors to neurons within the nucleus tractus solitarius and median preoptic nuclei that regulate blood pressure, metabolism, and fluid balance, as well as limbic and cortical areas known to impact stress responding and mood. The paraventricular nucleus of the hypothalamus did not display $\mathrm{AT}_{2}$ receptor-eGFP neurons, but efferent neurons terminating in the paraventricular nucleus and the GABA neurons surrounding the paraventricular nucleus did. The authors concluded those central $\mathrm{AT}_{2}$ receptors are positioned to regulate blood pressure, metabolism, and stress responses in their transgenic mouse model.

Behavioral effects reported in $\mathrm{AT}_{2}$ receptor null mice may be due to loss of $\mathrm{AT}_{2}$ receptor regulation in these areas. Although developmental apoptosis of mesenchymal cells is not altered in $\mathrm{AT}_{2}$ receptor null mice, an increased risk for renal diseases has been observed in $\mathrm{AT}_{2}$ receptor null mice (Kakuchi et al., 1995). The massive expression of $\mathrm{AT}_{2}$ receptor in the mesenchymal tissues was previously thought to regulate ontogeny of metanephros and tubulogenesis during development through apoptosis. However, nephrogenesis is not significantly altered by the $\mathrm{AT}_{2}$ receptor null condition. More detailed and long-term follow up studies have demonstrated that the loss of the $\mathrm{AT}_{2}$ receptor in mice may cause congenital anomalies of the kidneys and urinary tract (CAKUT syndrome) with a $>23 \%$ penetrance.

Deletion of the $\mathrm{AT}_{2}$ receptor in mice was also observed to cause inhibition of pressure natriuresis, vascular hypertrophy, and exacerbation of HF (Gross et al., 2000; Brede et al., 2001; Adachi et al., 2003). Renal vascular differentiation and the vascular smooth muscle contraction was altered because of the delayed expression of caldesmon and calponin, suggesting that the $\mathrm{AT}_{2}$ receptor enhances the differentiation of VSMCs and therefore plays an important role in vasculogenesis (Yamada et al., 1998). Role of $\mathrm{AT}_{2}$ receptor in cardiac, renal, and adrenal function is still unclear; its role in modulating pressure natriuresis is controversial as well (Keiser et al., 1992; Lo et al., 1995; Siragy and Carey, 1996). Pharmacological modulation of $\mathrm{AT}_{2}$ receptor with agonists or antagonists in rats suggested an antidiuretic and antinatriuretic function of the $\mathrm{AT}_{2}$ receptor. In rats and mice, $\mathrm{AT}_{2}$ receptor activation by the selective agonist $\mathrm{C} 21$ induced pressure natriuresis and lowered blood pressure (Kemp et al., 2014a). However, studies in $\mathrm{AT}_{2}$ receptor-knockout mice indicated exactly the opposite effects (Siragy, 2010).

Cardiac overexpression of the $\mathrm{AT}_{2}$ receptor in mice did not cause obvious morphologic or functional changes, but AngII infusion decreased blood pressure and produced a negative chronotropic effect (Masaki et al., 1998). Stimulation of bradykinin activity and nitric oxide production after inhibition of the $\mathrm{Na}^{+} / \mathrm{H}^{+}$ exchanger in $\mathrm{AT}_{2}$ receptor transgenic mice is attributed to this paradoxical phenotype (Tsutsumi et al., 1999).
Unequivocally defining the physiologic functions of the $\mathrm{AT}_{2}$ receptor through transgenic and knockout mouse models did not occur. However, the findings with these models as well as physiologic explanations have been controversial and raise questions regarding a "yin-yang" paradigm invoked to explain the regulatory roles of the two AngII receptor types.

\section{AGTR2 Genetic Polymorphism}

In humans, gene polymorphisms that persist in populations and cause variable phenotypes in the individuals are widely studied. Genotyping humans for functional, single nucleotide polymorphisms (SNPs) within the AGTR2 have been used in association studies to elucidate the pathogenic role of the $\mathrm{AT}_{2}$ receptor in cardiovascular, neurologic, and renal diseases in various populations. Many of nonsynonymous changes have been associated with X-linked mental retardation (e.g., G21V, R324Q, I337V, and I53F). In addition, a basepair deletion at position 395 causes a frame shift at $\mathrm{Phe}^{133}$ in the third transmembrane domain of the $\mathrm{AT}_{2}$ receptor, resulting in a truncated protein, which is also associated with intellectual deficit (Vervoort et al., 2002; Bienvenu et al., 2003; Renieri et al., 2005). The effect of the +1675 G/A SNP has been associated with left ventricular structural changes in young men with arterial hypertension (Schmieder et al., 2001). In addition, the intronic polymorphism $\mathrm{G}$ allele in patients was reported to modulate left ventricular mass under high sodium intake (Ott et al., 2007). In hypertrophic cardiomyopathy patients, there is an association between this polymorphism and hypertrophy, which could potentially be used as a marker for genetic predisposition to left ventricular hypertrophy (Carstens et al., 2011). A second SNP, A/C 3123, has been linked to metabolic disorders such as blood pressure and body mass index among the diabetic population in Japan (Miyaki et al., 2006; Kotani et al., 2007). Further analysis showed that this SNP could be a marker for glycemic control (i.e., via HbA1c level) among Japanese women (Kotani et al., 2009). Finally, a C to A conversion at position 4599 was linked to preeclampsia associated with body mass index $\geq 25 \mathrm{~kg} / \mathrm{m}$ (Dorn and Force, 2005), suggesting a gene-environment interaction (Zhou et al., 2013). Polymorphisms in intron 1 of the AGTR2 gene (A-1332G) occur with higher frequency in human patients with congenital urinary tract abnormalities, suggesting that the $\mathrm{AT}_{2}$ receptor may play an important role in the development of the urinary tract (Hohenfellner et al., 1999).

\section{E. Signaling}

The intracellular signal transduction processes activated by the $\mathrm{AT}_{2}$ receptor, which govern cellular and physiologic responses, are atypical for a GPCR and distinctly different from those mediated by the $\mathrm{AT}_{1}$ receptor (Fig. 7). Elucidating $\mathrm{AT}_{2}$ receptor-specific signaling 
pathways has been difficult and unsolved in most aspects, including receptor-proximal proteins that mediate the signaling by the $\mathrm{AT}_{2}$ receptor.

1. G-Protein Involvement. All of the classic motifs and signature residues of a GPCR are present in the $\mathrm{AT}_{2}$ receptor; however it fails to demonstrate classic features of G-protein signaling. Robust activation of heterotrimeric G-proteins, second messenger signals (calcium, DAG, cAMP, and $\mathrm{IP}_{3}$ ), desensitization by phosphorylation, and receptor regulation by internalization is not observed in $\mathrm{AT}_{2}$ receptor transfected cells (Kambayashi et al., 1993; Mukoyama et al., 1993; Miura and Karnik, 2002) or the native cells R3T3, PC12W, ovarian granulosa cells (Dudley et al., 1991; Pucell et al., 1991; Bottari et al., 1992a; Leung et al., 1992a; Webb et al., 1992). This behavior is consistent with observations of affinity of AngII binding, which is not affected by GTP or its analog GTP $\gamma \mathrm{S}$ in tissues exclusively expressing the $\mathrm{AT}_{2}$ receptor, such as human myometrium, rat adrenal glands, and bovine cerebellar cortex (Bottari et al., 1992a). Lack of agonist-induced desensitization and recycling of the $\mathrm{AT}_{2}$ receptor is also observed in adult mice (Unger, 1999).

Multiple reports suggesting potential $\mathrm{AT}_{2}$ receptor coupling to $G_{i} / G_{o}$ have directly linked downstream signals to activation of this class of G-proteins (Fig. 7). For instance, $\mathrm{AT}_{2}$ receptor s sensitive to $\mathrm{GTP} \gamma \mathrm{S}$ and pertussis toxin have been described in the locus coeruleus of the rat brain and thalamic or geniculate nuclei implying a coupling to heterotrimeric G-proteins, $\mathrm{G}_{\mathrm{i}}$ and $\mathrm{G}_{\mathrm{o}}$ (Tsutsumi and Saavedra, 1991). The $\mathrm{AT}_{2}$ receptors appear to bind to $\mathrm{Gi}_{\alpha 2}$ or $\mathrm{Gi}_{\alpha 3}$ in developing fetus in rat (Zhang and Pratt, 1996) and in adult rat kidney cells. The intracellular third loop (ICL3) of the $\mathrm{AT}_{2}$ receptor was shown to be important for its coupling to $G_{i}$ (Hayashida et al., 1996). Evidence of ICL3 involvement was also shown in $\mathrm{AT}_{2}$ receptor induction of apoptotic responses in the PC12W neuronal lineage cells (Lehtonen et al., 1999) and in $\mathrm{AT}_{2}$ receptor-mediated inhibition of $\mathrm{IP}_{3}$ generation in Xenopus oocytes (Kumar et al., 2002). More recent studies implicated the involvement of $\mathrm{G}_{\mathrm{i}}$ in $\mathrm{AT}_{2}$ receptor-dependent increases in nitric oxide synthase expression (Li et al., 2007a) and the inhibition of proximal tubule $\mathrm{Na}^{+}$-ATPase by $\mathrm{Ang}(1-7)$ (Siragy and Carey, 1996; Siragy et al., 1996; Gohlke et al., 1998; Siragy, 2000). The production of prostacyclin in differentiated adipocytes was blocked by PD123177 and not by losartan, which suggests that this is an $\mathrm{AT}_{2}$ receptor-mediated signal (Darimont et al., 1994). Whether this mechanism involved a G-protein, a $\mathrm{G}_{\mathrm{i}}$ signaling pathway remains to be clarified. Direct G-protein activation assays in heterologous expression systems remain difficult with the $\mathrm{AT}_{2}$ receptor. Whether ICL3 is important for coupling of the full-length receptor to $G_{i}$ has not been validated by mutations in ICL3. Discrepant findings regarding G-protein coupling with the $\mathrm{AT}_{2}$ receptor is an unresolved research challenge that constrains a full understanding of $\mathrm{AT}_{2}$ receptor signaling mechanisms.

2. Protein Phosphatase Involvement. Depending on the tissues, activation of the $\mathrm{AT}_{2}$ receptor can stimulate protein phosphatases (MKP-1, PP2A, SHP-1) and protein

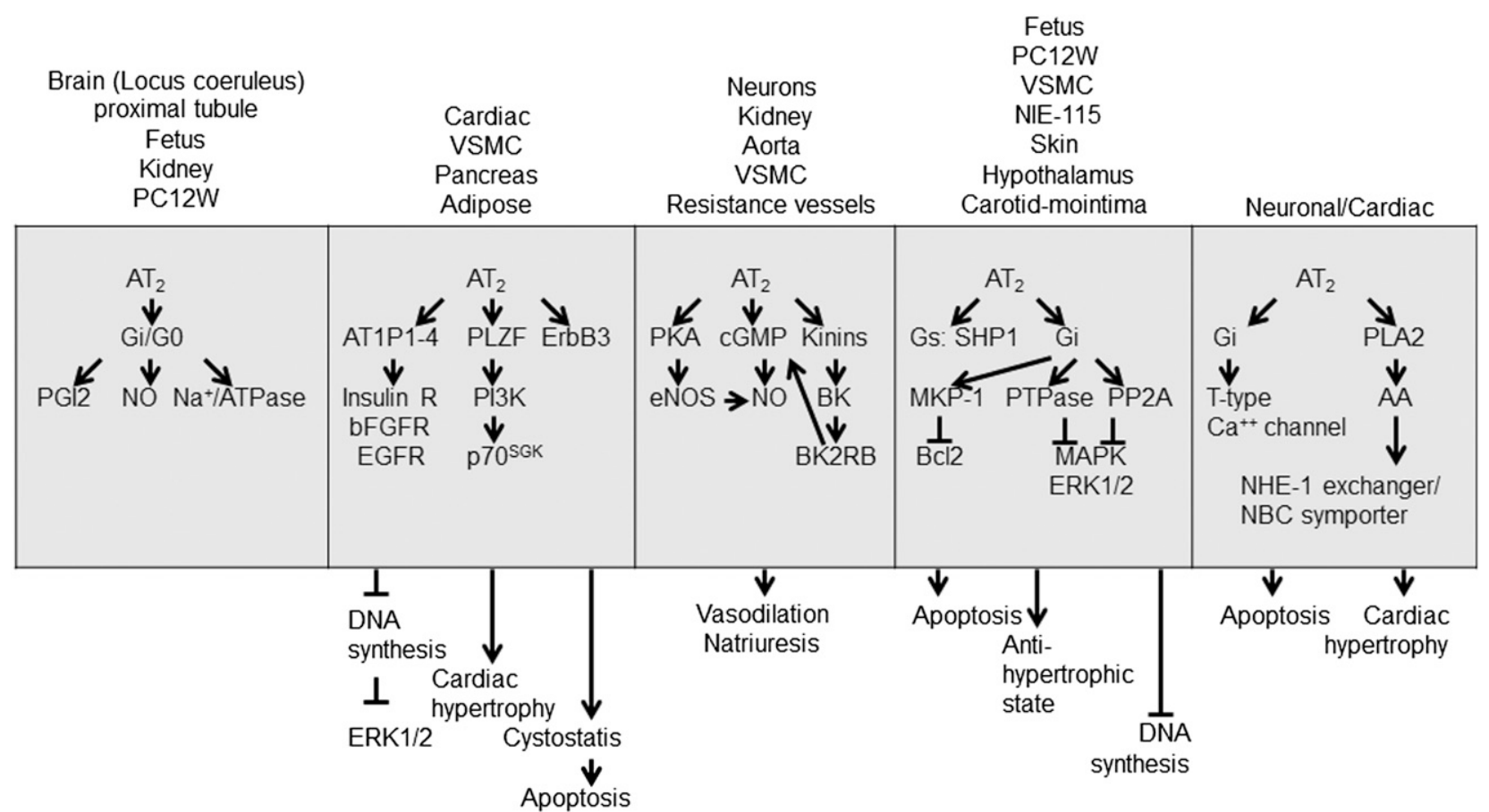

Fig. 7. Diverse signaling by $\mathrm{AT}_{2}$ receptor. Each tile represents signaling pathways reported in different cellular and tissue context leading to physiologic or pathologic consequences. See main text for details. 
dephosphorylation (Fig. 7). The activation of vanadatesensitive tyrosine phosphatase as well as Ser/Thr phosphatases has emerged as a key mechanism accounting for the antigrowth and apoptotic effects of the $\mathrm{AT}_{2}$ receptor (Bottari et al., 1992a; Horiuchi et al., 1997; Elbaz et al., 2000). The activation of the protein tyrosine phosphatase SHP-1 mediated by $\mathrm{AT}_{2}$ receptor is pertussis toxin insensitive, thus a $\mathrm{G}_{\mathrm{i}}$-independent signal (Bedecs et al., 1997). SHP-1 coupling may actually involve an atypical G-protein scaffolding mechanism, $\mathrm{G}_{\beta \gamma}$-independent constitutive association of the receptor with $\mathrm{G}_{\mathrm{s}}$ and SHP-1 (Feng et al., 2002). In contrast to these studies, a $\mathrm{G}_{\mathrm{i}}$-coupled activation of PTP by $\mathrm{AT}_{2}$ receptor in VSMCs was reported. Activation of $\mathrm{G}_{\mathrm{i}} / \mathrm{G}_{\mathrm{o}}$ in these cells by a peptide corresponding to the third intracellular loop of the $\mathrm{AT}_{2}$ receptor was inhibited by pertussis toxin, as well as by sodium orthovanadate a tyrosine phosphatase inhibitor. The serine/threonine phosphatase inhibitor okadaic acid did not block it (Hayashida et al., 1996). Similarly, activation of soluble SH-PTP1 to attenuate MAPK activation and DNA synthesis through thymidine incorporation observed in NIE-115 neuronal cells was suggested to be $G_{i} / G_{o}$ dependent.

Activation of PTPases by the $\mathrm{AT}_{2}$ receptor in cells may limit mitogen-activated protein kinase (MAPK) signaling and dephosphorylation of extracellular signalregulated kinases 1 and 2 (ERK1/2) (Huang et al., 1995; Hayashida et al., 1996; Bedecs et al., 1997; Fischer et al., 1998; Akishita et al., 1999). The $\mathrm{AT}_{2}$ receptor is a generalized negative regulator of intracellular kinase signaling, and thus it may antagonize growth stimulation by AngII, EGF, PDGF, or serum. $\mathrm{AT}_{2}$ receptor knockout mice confirm this aspect, because elevated levels of ERK1/2 were reported at baseline and in response to serum (Akishita et al., 1999). ERK1/2 dephosphorylation may occur through three phosphatases: SHP-1, mitogen-activated protein kinase phosphatase 1 (MKP-1), and protein phosphatase 2A (PP2A) (Huang et al., 1995; Horiuchi et al., 1997; Yang et al., 1999). The $\mathrm{AT}_{2}$ receptor-mediated suppression of MAPK activation in vessels is reported (Nakajima et al., 1995). The carotid artery expresses low levels of the $\mathrm{AT}_{2}$ receptor (Viswanathan and Saavedra, 1992), but re-expressed $\mathrm{AT}_{2}$ receptor at the edge of neointima during carotid injury or in a healing wound of the skin suppresses AngII-induced MAPK activity in a PD123319-sensitive manner to facilitate remodeling of these tissues (Nakajima et al., 1995). $\mathrm{AT}_{2}$ receptor activation is linked to upregulation of SHP-1 in vascular-targeted $\mathrm{AT}_{2}$ receptor transgenic mice (Matsubara et al., 2001). The $\mathrm{AT}_{2}$ receptor in the neonatal hypothalamic neurons is reported to inactivate MAPK, and the presence of the $\mathrm{AT}_{2}$ receptor antagonist PD123319 enhanced MAPK activity in these neurons. The ERK inactivated by the $\mathrm{AT}_{2}$ receptor plays a physiologic role in vivo, depending upon the biologic context in which $\mathrm{AT}_{2}$ receptor is engaged. For instance, in PC12W cells, which express only $\mathrm{AT}_{2}$ receptor, AngII-regulated short-lived ERK phosphorylation plays a role in neuronal differentiation by AngII and nerve growth factor costimulation (Stroth et al., 2000). In the heart of $\mathrm{AT}_{2}$ receptor transgenic mice, the antihypertrophic state of myocytes is associated with ERK dephosphorylation, which indicates that ERK inactivated by the $\mathrm{AT}_{2}$ receptor plays a physiologic role in vivo (Masaki et al., 1998).

Conflicting reports also exist regarding phosphatase activation in $\mathrm{AT}_{2}$ receptor-mediated proapoptotic effects. Dephosphorylation of Bcl-2 by MKP-1 was observed in $\mathrm{AT}_{2}$ receptor-induced apoptosis in PC12W cells, which was ligand dependent (Horiuchi et al., 1997); apoptosis was shown to be a constitutive function of the $\mathrm{AT}_{2}$ receptor that involves activation of p38 MAPK (Miura and Karnik, 2000). Overexpression of the $\mathrm{AT}_{2}$ receptor in neonatal cardiomyocytes promoted growth (D'Amore et al., 2005). Overexpression and AngII stimulation of the $\mathrm{AT}_{2}$ receptor in porcine cardiac fibroblasts inhibited protein tyrosine phosphatases (Warnecke et al., 2001). Observed contradictions further highlight the context-specific effects of the $\mathrm{AT}_{2}$ receptor overexpression.

3. Scaffolding Protein Involvement. Choosing an unbiased approach to understanding how the $\mathrm{AT}_{2}$ receptor suppresses cellular growth in different contexts yielded intriguing leads (i.e., yeast 2-hybrid "fishing" with the $\mathrm{AT}_{2}$ receptor C-terminal tail as bait), which are indicative of completely novel signaling modalities (Fig. 7); however, these are in-progress studies that might lead to novel physiologic endpoints.

Negative regulation of several receptor tyrosine kinases (RTKs) including fibroblast growth factor, epidermal growth factor (Zhang et al., 2009) and insulin receptors by $\mathrm{AT}_{2}$ receptor is described as transinactivation by $\mathrm{AT}_{2}$ receptor activation of PTPases as well as inhibition of autophosphorylation of the RTKs (Elbaz et al., 2000; De Paolis et al., 2002). Possible mechanisms suggested for the transinactivation of RTKs include a direct physical interaction between the two receptors. The ErbB3 EGF receptor as an interacting partner with $\mathrm{C}$ terminus of the $\mathrm{AT}_{2}$ receptor was identified. These authors also showed that replacing ICL3 of the $\mathrm{AT}_{2}$ receptor with that of $\mathrm{AT}_{1}$ receptor abolishes the interaction with ErbB3 and the inhibitory effects on cell proliferation and the activation of apoptosis (Pulakat et al., 1998, 2002).

The $\mathrm{AT}_{2}$ receptor $\mathrm{C}$-terminal tail interaction with the transcription factor promyelocytic zinc finger protein (PLZF) is one of the most surprising discoveries. After AngII stimulation, PLZF translocates from the cytosol to the plasma membrane and then promotes internalization of the $\mathrm{AT}_{2}$ receptor with PLZF, leading to accumulation of $\mathrm{AT}_{2}$ receptor in the perinuclear membrane. PLZF enters the nucleus where it activates the p85 $\alpha$ subunit gene of PI3K and enhances $\mathrm{p} 70^{\mathrm{S} 6}$ kinase activity, which is essential for protein synthesis 
(Senbonmatsu et al., 2003). The finding that $\mathrm{AT}_{2}$ receptor internalizes when associated with PLZF is an interesting phenomenon, given that $\mathrm{AT}_{2}$ receptor does not normally internalize (Mukoyama et al., 1995; Turu et al., 2006). PLZF is a highly expressed transcription factor in the heart; however, its expression is not affected in the $\mathrm{AT}_{2}$ receptor-knockout mice. $\mathrm{AT}_{2}$-knock-out mice do not show a hypertrophic response to pressure overload after aortic banding, this phenotype is associated with a failure to upregulate $\mathrm{p} 70^{\mathrm{S} 6}$ kinase after pressure overload. The $\mathrm{AT}_{2}$ receptor-null hearts also fail to activate $\mathrm{p} 85 \alpha$ transcription in response to AngII infusion. These data strongly suggest that the $\mathrm{AT}_{2}$ receptor-PLZF-p85 $\alpha-\mathrm{p} 70^{\mathrm{S} 6}$ kinase-signaling axis might be important in the induction of cardiac hypertrophy (Senbonmatsu et al., 2000).

Two independent groups identified ATIP/ATBP50 $\left(\mathrm{AT}_{2}\right.$ receptor interacting protein) (Nouet et al., 2004; Wruck et al., 2005). At least four members are known to date for the ATIP-family (ATIP1-4). They all conserve an $\mathrm{AT}_{2}$ receptor-interacting domain and can inhibit insulin, epidermal growth factor, and basic fibroblast growth factor-induced ERK1/2 activation and DNA synthesis in CHO cells in a similar manner to $\mathrm{AT}_{2}$ receptor (Nouet et al., 2004). The ATIP-mediated inhibition of ERK1/2 requires $\mathrm{AT}_{2}$ receptor expression but not treatment with AngII (Nouet et al., 2004). Li et al. (2007b) showed that siRNA-mediated knockdown of ATIP inhibits $\mathrm{AT}_{2}$ receptor-mediated expression of methane methylsulfonate-sensitive 2, which plays an important role in the ubiquitin proteasome system and DNA repair. Methane methylsulfonate-sensitive 2 expression is increased in occluded/remodeling cerebral arteries, which is consistent with a protective role for the $\mathrm{AT}_{2}$ receptor in brain injury. The siRNA-mediated knockdown of ATIP is also reported to reduce cell surface expression of the $\mathrm{AT}_{2}$ receptor and suppress the antiproliferative effect (Wruck et al., 2005).

Although these studies yielded novel and interesting directions to pursue, the rationale for choosing the $\mathrm{AT}_{2}$ receptor C-terminal tail as bait is not fully justified. There is no evidence to suggest that the $\mathrm{AT}_{2}$ receptor C-terminal tail mediates the functions ultimately described for each scaffold protein. Rather surprisingly, involvement of $\mathrm{G}_{\mathrm{s}} / \mathrm{G}_{\mathrm{i}} / \mathrm{G}_{\mathrm{o}}$ proteins and the ICL3 appear to be an important determinant of $\mathrm{AT}_{2}$ receptor coupling to PLZF as well as for interactions with ErbB3. These findings again point to G-protein requirement at least in unconventional mode and raise doubts about the mechanisms of scaffold signaling by the $\mathrm{AT}_{2}$ receptor .

4. Nitric Oxide/cGMP Involvement. Nitric oxide (NO)stimulated soluble guanylyl cyclase catalytic activity generates cGMP to generally exert protective effects in various tissues (Toda et al., 2007). Initial in vitro studies in neuronal cell lines suggested that AngII via the $\mathrm{AT}_{2}$ receptor reduced cGMP levels in neuronal cells (Sumners and Myers, 1991; Sumners et al., 1991; Bottari et al., 1992b; Brechler et al., 1993). In other in vitro studies cGMP was not detected (Leung et al., 1992b; Webb et al., 1992; Mukoyama et al., 1993, 1995; Siragy and Carey, 1996). The studies by Liu et al. (1997) demonstrated that beneficial effects of $\mathrm{AT}_{2}$ receptor involved kinin stimulation and cGMP production. Gohlke et al. (1998) also showed that $\mathrm{AT}_{2}$ receptor stimulation increased cGMP levels in the rat aorta, which was further corroborated in $\mathrm{AT}_{2}$ receptor-null mice studies (Siragy et al., 1999). The $\mathrm{AT}_{2}$ receptor transgenic mice have elevated levels of cGMP in the aorta (Tsutsumi et al., 1999).

Whether the $\mathrm{AT}_{2}$ receptor directly couples to NO/cGMP system especially in $\mathrm{AT}_{2}$ receptor transgenic and knockout mice or through an indirect bradykinin B2-receptor dependent mechanism has been controversial. Tsutsumi et al. (1999) showed that the $\mathrm{AT}_{2}$ receptor stimulates bradykinin production in VSMCs, and AngII-mediated cGMP response could be blocked with the bradykinin receptor antagonist. $\mathrm{AT}_{2}$ receptor stimulates bradykinin production and promotes the NO/cGMP pathway in a paracrine manner, and this mechanism is important in $\mathrm{AT}_{2}$ receptor-mediated vasodilatation of human coronary arteries (Batenburg et al., 2004). Different signaling circuitry may be involved in $\mathrm{AT}_{2}$ receptor-dependent NO/cGMP production. In the thoracic abdominal aortic constriction mice model, $\mathrm{AT}_{2}$ receptor activation induces phosphorylation of eNOS via a PKA-mediated signaling pathway (Yayama et al., 2006). Bradykinin acting on the bradykinin $\mathrm{B}_{2}$ receptor can also induce PKA-dependent phosphorylation of eNOS. Evidences for a functional heterodimerization of the $\mathrm{AT}_{2}$ receptor and $B_{2} R$ receptors have been reported, which suggest that these receptors may physically associate and possibly increase NO production (Abadir et al., 2006).

5. Ion-Channel Protein Involvement. A highly reproducible signal from the $\mathrm{AT}_{2}$ receptor seems to be coupling to ion-channel proteins (Fig. 7), such as the hypothalamic neuronal delayed rectifier potassium channel (Kang et al., 1993, 1994), the T-type calcium channel (Buisson et al., 1992, 1995) in other cells, which can suppress or induce cellular growth and differentiation (Nakajima et al., 1995; Stoll et al., 1995; Laflamme et al., 1996; Meffert et al., 1996; Munzenmaier and Greene, 1996; Tsuzuki et al., 1996a,b; Gallinat et al., 1998; Stroth et al., 1998; Cote et al., 1999; Gendron et al., 1999) as well as support apoptosis (Yamada et al., 1996; Chamoux et al., 1999; Gallinat et al., 1999).

Cardiac ventricular myocytes express both the $\mathrm{AT}_{1}$ receptor and $\mathrm{AT}_{2}$ receptor. Expression of $\mathrm{AT}_{1}$ receptor along with $\mathrm{AT}_{2}$ receptor is increased in the hypertrophic ventricles of SHR and two-kidney one-clip hypertensive rats (Suzuki et al., 1993; Busche et al., 2000). AngIIstimulated arachidonic acid production in cardiac myocytes is fully blocked by the $\mathrm{AT}_{2}$ receptor antagonist PD123317, suggesting that $\mathrm{AT}_{2}$ receptor mediates the activation of phospholipase A2 (Lokuta et al., 1994). The 
$\mathrm{AT}_{2}$ receptor-arachidonic acid signal activates the $\mathrm{Na} / \mathrm{HCO}$ symporter system, which maintains $\mathrm{pH}$ in myocytes. The arachidonic acid-induced symporter activation in the heart is a unique function of the $\mathrm{AT}_{2}$ receptor (Sandmann et al., 1998). In rats after myocardial infarction, the postinfarct upregulation of the Na1/H1-exchanger (NHE-1) and NBC ion transporter systems are differentially regulated by $\mathrm{AT}_{1}$ and $\mathrm{AT}_{2}$ receptors. ACE inhibitors prevent this regulation in heart tissue. However, the $\mathrm{AT}_{1}$ receptor antagonists block NHE-1 upregulation, and the $\mathrm{AT}_{2}$ receptor antagonists block the increase of the NBC (Horiuchi et al., 1999; de Gasparo et al., 2000; Nouet and Nahmias, 2000). This difference may be important in evaluating therapeutic intervention.

6. Involvement of Constitutive Activity. Ligandindependent actions of the $\mathrm{AT}_{2}$ receptor (Miura and Karnik, 2002) is recognized as an important mode of its in vivo function in several scenarios. Upregulated $\mathrm{AT}_{2}$ receptor expression as seen in remodeling adult tissues and developing fetal tissue can initiate constitutive signal transduction without AngII stimulation, leading to apoptosis (Miura and Karnik, 2000). Stimulation of apoptosis in prostate cancer cells was mediated by increased expression of the $\mathrm{AT}_{2}$ receptor (Li et al., 2009b). $\mathrm{AT}_{2}$ receptor gene transfer in the same cells mediated increased expression of bradykinin and iNOS in VSMCs. Downregulation of the $\mathrm{AT}_{1}$ receptor was also observed by the same treatment (Jin et al., 2002). In another gene transfer study, Porrello et al. (2009) found that the $\mathrm{AT}_{2}$ receptor constitutively antagonized $\mathrm{AT}_{1}$ receptor-mediated cardiomyocyte autophagy. Homooligomerization of the $\mathrm{AT}_{2}$ receptor through disulfide exchange between $\mathrm{Cys}^{35}$ in one $\mathrm{AT}_{2}$ receptor and $\mathrm{Cys}^{290}$ in its dimerization partner was shown to be important for induction of apoptosis without AngII stimulation (Miura et al., 2005). Augmented release of bradykinin in mouse coronary artery endothelial cells was constitutive function (Zhu et al., 2010). Repression of ERK activity mediated by ATIP requires $\mathrm{AT}_{2}$ receptor expression but not activation by AngII (Nouet et al., 2004). Feng et al. (2002) provided a potential mechanism for the constitutive function of the $\mathrm{AT}_{2}$ receptor. They documented that constitutive association of $\mathrm{G}_{\alpha}$ with SHP-1 and the $\mathrm{AT}_{2}$ receptor is $\mathrm{G}_{\beta \gamma}$ independent and this association is essential in $\mathrm{AT}_{2}$ receptor-mediated ITIM-independent activation of SHP-1.

At the cellular level, a clear mechanistic model of $\mathrm{AT}_{2}$ receptor signal transduction and cell physiology has yet to emerge. Despite intensive investigation, the $\mathrm{AT}_{2}$ receptor is one of the inadequately understood components of the renin-angiotensin system.

\section{F. Expression and Regulation}

Expression of the $\mathrm{AT}_{2}$ receptor is regulated in vivo in response to environmental cues. Both transcriptional and translational regulation seems to take place, but low $\mathrm{AT}_{2}$ receptor expression levels are maintained in normal nongrowing cells. Growth factors, inflammatory mediators, and the growth phase of the cells regulate the $\mathrm{AT}_{2}$ receptor gene. Extended serum depletion combined with insulin or IGF-1 or interleukin-1b (IL-1b) stimulate the expression of $\mathrm{AT}_{2}$ receptor in VSMCs, whereas growth factors, like PDGF and phorbol ester, inhibit expression of $\mathrm{AT}_{2}$ receptor (Kambayashi et al., 1993, 1996). The plasma insulin concentrations regulate $\mathrm{AT}_{2}$ receptor expression in aorta (Kambayashi et al., 1996). Tissue-specific expression of the $\mathrm{AT}_{2}$ receptor has been traced to enhancer elements in the $\mathrm{AT}_{2}$ receptor gene promoter consisting of the AP-1 (inhibitor), C/EBP, NF/IL-6, IRS, and interferon regulatory factor IRF-2 (activator).

1. Developmental Regulation. Human tissue expression of $\mathrm{AT}_{2}$ receptor has been documented by immunocytochemistry, reverse transcriptase-polymerase chain reaction, and microarray analyses (Ichiki and Inagami, 1995; de Gasparo et al., 2000; Herradon et al., 2004; Kim et al., 2005b; Chakrabarty et al., 2008; Petracco et al., 2012). In most tissues, the $\mathrm{AT}_{1}$ receptor and $\mathrm{AT}_{2}$ receptor coexist. The $\mathrm{AT}_{2}$ receptor mRNA expression analysis provided a better picture of the tissue- and species-specific distribution (Kakuchi et al., 1995; Shanmugam et al., 1995; Johren and Saavedra, 1996). The $\mathrm{AT}_{2}$ receptor is abundantly and ubiquitously expressed in the developing rat fetus and neonatal tissues, which lead to early speculation of a developmental role for the $\mathrm{AT}_{2}$ receptor (Suzuki et al., 1993; Bastien et al., 1996).

In mouse fetal mesenchymal cells, $>95 \%$ of the AngII binding is due to the $\mathrm{AT}_{2}$ receptor. Regulation of its expression in relation to fetal developmental stages may suggest a potential role for this receptor in developmental processes. $\mathrm{AT}_{2}$ receptor expression levels increase at embryonic days E11-E13. Maximal $\mathrm{AT}_{2}$ receptor expression levels are seen on E19 followed by a rapid decline in the newborn animals to undetectable expression. The $\mathrm{AT}_{2}$ receptor-null genotype has only a marginal effect, if any, on the development of mesenchyme-rich tissue or organs in the mouse such as skin, tongue, kidney, or adrenal (Kakuchi et al., 1995). However, a high incidence of urological abnormalities (Graham et al., 1997) and delay in vasculogenesis was reported in $\mathrm{AT}_{2}$ receptor null mice (Yamada et al., 1998). The $\mathrm{AT}_{2}$ receptor is the exclusive AngII receptor in the fetal rat aorta, which decreases by $\sim 85 \%$ at 2 weeks of age and to $25 \%$ at 8 weeks of age (Viswanathan and Saavedra, 1992). $\mathrm{AT}_{2}$ receptor blockade with PD123319 from E16 to E21 significantly decreases DNA synthesis in the developing aorta (Nakajima et al., 1995), and the $\mathrm{AT}_{2}$ receptor-null mice have reduced levels of the VSMC differentiation markers calponin and caldesmon up to 4 weeks after birth (Yamada et al., 1999). In a similar fashion, stimulating the $\mathrm{AT}_{2}$ receptor induces neurite outgrowth and regulates neurofilament expression in neural cells (Laflamme et al., 1996) as well as 
promotes apoptotic effects neuronal cell lines (Yamada et al., 1996; Horiuchi et al., 1997). Increase in neuronal cell number in certain brain structures associated with central neurologic abnormalities, learning, and memory deficit have been reported for $\mathrm{AT}_{2}$ receptor knockout mice (Hein et al., 1995; Ichiki et al., 1995; von Bohlen und Halbach et al., 2001). Whether the increase in cell number in these mice is due to increased neuronal proliferation or a suppression of apoptosis is not clear. The proapoptotic effect of the $\mathrm{AT}_{2}$ receptor is not found in the cardiomyocytes of $\mathrm{AT}_{2}$ receptor transgenic mouse (Sugino et al., 2001).

2. Adult Tissue Regulation. Receptor expression declines after birth, but the $\mathrm{AT}_{2}$ receptor is expressed at low levels in the normal adult cardiovascular system, adrenal gland, kidney, brain, uterine myometrium, and skin. The capacity for regulated changes in expression of $\mathrm{AT}_{2}$ receptor is retained in many adult tissues and often in remodeling tissues, a finding that suggests a potential role for this receptor in human cardiovascular disease as well.

The expression of both $\mathrm{AT}_{1}$ receptor and $\mathrm{AT}_{2}$ receptor in the adrenal gland, nonpregnant uterus, ovarian follicular granulosa cells, and cerebellar cortex is conserved in all mammals including humans (David, 1976; Whitebread et al., 1989; Criscione et al., 1990; Bottari et al., 1991; Pucell et al., 1991; Cox et al., 1993). There are some examples of tissues that contain more $\mathrm{AT}_{2}$ receptor than $\mathrm{AT}_{1}$ receptor. In the uterus, for example, expression of $\mathrm{AT}_{2}$ and $\mathrm{AT}_{1}$ receptors changes reversibly depending on pregnancy and parturition in human and sheep (Cox et al., 1993). The expression switching from high $\mathrm{AT}_{2}$ receptor levels to a low-level $\mathrm{AT}_{2}$ receptor and to completely $\mathrm{AT}_{1}$ receptor is a fascinating developmental phenomenon that is not clearly understood.

The $\mathrm{AT}_{2}$ receptor density increases in tissues under pathologic conditions in which inflammation and tissue remodeling occur. Such changes have been observed in tissues, such as skin wound, balloon-catheterized vasculature, infarcted myocardium, and ischemic brain during pathogenesis of hypertension, atherosclerosis, diabetes mellitus, nephropathy, and pulmonary fibrosis (Booz and Baker, 1996; Lemarie and Schiffrin, 2010). The observed increase in $\mathrm{AT}_{2}$ receptor expression during pathology can be viewed as fetal gene reactivation, which is a prominent feature of various disease states, and this response may indicate that the $\mathrm{AT}_{2}$ receptor has a definitive regulatory function in these instances (Paul et al., 2006). In rodents, $\mathrm{AT}_{2}$ receptor expression is upregulated in $\mathrm{HF}$ (Ohkubo et al., 1997; Steckelings et al., 2005) and is up- and downregulated in a temporally dependent manner postinfarction (Nio et al., 1995; Lax et al., 2004). Expression of $\mathrm{AT}_{2}$ receptors in humans is less clear. A decrease in $\mathrm{AT}_{2}$ receptor expression during $\mathrm{HF}$ was reported (Matsumoto et al., 2000), whereas, an increase in expression was seen during atrial fibrillation (Goette et al., 2000). Myocardial
$\mathrm{AT}_{2}$ receptor expression is upregulated in patients with dilated cardiomyopathy. In the nonfailing human heart $\sim 40 \%$ of AngII receptors are $\mathrm{AT}_{2}$ receptor (Tsutsumi et al., 1998), and the $\mathrm{AT}_{2}$ receptor constitutes $50-70 \%$ of angiotensin binding sites in the adult myocardium in humans (Regitz-Zagrosek et al., 1995). In the adult rodent myocardium, $\sim 10 \%$ of adult cardiomyocytes express $\mathrm{AT}_{2}$ receptor (Busche et al., 2000; Steckelings et al., 2005), but $\mathrm{AT}_{1}$ receptor blockade increases $\mathrm{AT}_{2}$ expression levels in rodents. Hence, under conditions of $\mathrm{AT}_{1}$-inhibitor therapy, circulating AngII levels increase, creating the potential for increased $\mathrm{AT}_{2}$ receptor function and facilitation of therapeutic tissue remodeling. This is the basis for targeting $\mathrm{AT}_{2}$ receptors for therapy in patients treated with $\mathrm{AT}_{1}$ receptor blockers to treat hypertension and HF. Adult brain $\mathrm{AT}_{2}$ receptor may regulate central effects of angiotensin peptides in osmoregulation, cognitive functions, and behavioral functions (Hein et al., 1995; Hohle et al., 1995). Additionally, the role of the $\mathrm{AT}_{2}$ receptor in regeneration and protection of neuronal tissue has been suggested (Steckelings et al., 2011a).

In the vasculature, a ratio of $80 \% \mathrm{AT}_{1}$ receptor to $20 \%$ $\mathrm{AT}_{2}$ receptor exists in coronary endothelial cells derived from spontaneously hypertensive rats (SHR) (Stoll et al., 1995). Adult vasculature has low levels of $\mathrm{AT}_{2}$ receptor, which may increase the sensitivity of vasculature to AngII when an $\mathrm{AT}_{2}$ receptor-specific antagonist is present via the vascular $\mathrm{AT}_{1}$ receptor (Verlander et al., 2011). The inhibitory effect of the $\mathrm{AT}_{2}$ receptor on neointima formation after balloon catheterization of carotid artery has been extensively studied. The growth of the neointima was reduced by the $\mathrm{AT}_{2}$ receptor antagonist PD123319 (Nakajima et al., 1995). Chronic AngII infusion increased the wall thickness-to-lumen ratio of mesenteric arteries associated with increase of both $\mathrm{AT}_{1}$ receptor and $\mathrm{AT}_{2}$ receptor (Cao et al., 1999). End-stage ischemic heart disease and dilated cardiomyopathy increased the density of the $\mathrm{AT}_{2}$ receptor in endocardial, interstitial, perivascular, and infarcted regions of the ventricle of patients. That $\mathrm{AT}_{2}$ receptor $\mathrm{s}$ may play a role in vessel formation is suggested by rich microvessels found in the border zone between noninfarcted and infarcted myocardium (Wharton et al., 1998).

In the kidney, 10 to $20 \%$ of total AngII receptors are $\mathrm{AT}_{2}$ receptor, and no $\mathrm{AT}_{2}$ receptors are detected in the rat renal cortex and glomeruli. Dietary sodium may modulate expression levels of $\mathrm{AT}_{2}$ receptor in glomerular and interstitial tissue. The $\mathrm{AT}_{2}$ receptor is present in large preglomerular vessels of the renal cortex and in the tubular interstitial cells in human kidney (Chansel et al., 1993; Goldfarb et al., 1994; Kaufman et al., 1999; Carey et al., 2001).

\section{G. Pathophysiological Aspects of AngII Type 2 Receptor Activation}

In the last 10 years, strides have been made toward understanding the pathologic and physiologic roles of 
the $\mathrm{AT}_{2}$ receptor (Knowle et al., 2000; Miura and Karnik, 2000; De Paolis et al., 2002). The $\mathrm{AT}_{2}$ receptor has been implicated in cardiovascular disease as a beneficial moiety due to its proposed functions that, overall, appear to counterbalance the actions of the $\mathrm{AT}_{1}$ receptor (Berk, 2003; Miura et al., 2010). Activation of the $\mathrm{AT}_{2}$ receptor inhibits autophagy mediated by the $\mathrm{AT}_{1}$ receptor in cardiomyocytes (Porrello et al., 2009). Overall, the $\mathrm{AT}_{2}$ receptor may play a role in several $\mathrm{AT}_{1}$ receptor-independent biologic processes as well (RuizOrtega et al., 2000, 2001a; Mifune et al., 2000; Weidekamm et al., 2002; Benndorf et al., 2003; Gingras et al., 2003; Caballero et al., 2004; Zhao et al., 2005b; Zhu et al., 2000; Mertens et al., 2010). Notably, activation of the $\mathrm{AT}_{2}$ receptor in cells of neuronal origin, induced neurite outgrowth, and elongation mediated cellular excitability and migration and, in some cases, caused neuronal cell death (Guimond and Gallo-Payet, 2012). Taken together, it has become clear that the signaling mechanisms of the $\mathrm{AT}_{2}$ receptor are diverse and require further studies to fully elucidate the role of this receptor in biologic processes.

1. Regulation of Vascular Response. Physiologic effects of $\mathrm{AT}_{2}$ receptor function in the vasculature are complex and can be both vasoconstricting and vasodilatating depending on the context. $\mathrm{AT}_{2}$ receptor activation causes vasodilatation in most isolated arteries and it exerts a vasodepressor effect in vivo, when a concomitant, low-dose AT1R blockade is also administered (Widdop et al., 2003). Actions of the $\mathrm{AT}_{2}$ receptor antagonist PD123319 and agonist CGP42112 consistent with this expectation have been demonstrated in rodent arteries as well as in human coronary microarteries (Carey et al., 2001; Batenburg et al., 2004). The pressor response to AngII is greater in $\mathrm{AT}_{2}$ receptornull mice than normal controls. VSMC-targeted overexpression of $\mathrm{AT}_{2}$ receptor in transgenic mice causes vasodilatation in vivo (Tsutsumi et al., 1999). That the $\mathrm{AT}_{2}$ receptor also mediates vasoconstriction in mesenteric resistance arteries of SHR and senescent rats is a recent finding (You et al., 2005). Antihypertensive treatment of 4 weeks restored $\mathrm{AT}_{2}$ receptor expression and vasodilator function in SHR resistance arteries. Pinaud et al. (2007) reported that the $\mathrm{AT}_{2}$ receptor induces a vasodilator effect in young rats and vasoconstrictive effects in old rats. Resolving the apparently conflicting roles of the $\mathrm{AT}_{2}$ receptor in different vascular beds should be a focus for future studies.

2. Regulation of Cardiac Growth Response. The $\mathrm{AT}_{1}$ receptor induces mitogenic effects in many tissues and cell types. Regulation of cell proliferation by the $\mathrm{AT}_{2}$ receptor through an antiproliferative action opposed to the $\mathrm{AT}_{1}$ receptor activation first discovered in coronary endothelial cells is also observed in microvascular endothelial cells, VSMCs, neuronal cells, pheochromacytoma cells, and fibroblasts (Stoll et al., 1995; Steckelings et al., 2005). The potential clinical ramifications of an $\mathrm{AT}_{2}$ receptor-agonist therapy for the treatment of proliferative pathologies including cardiac fibrosis are vast. But the literature reports are confusing, for instance, $\mathrm{AT}_{1}$ receptor-dependent stimulation of DNA and protein synthesis by AngII in neonatal cardiomyocytes and cardiac fibroblasts is observed only when the $\mathrm{AT}_{2}$ receptor-selective antagonist $\mathrm{PD} 123319$ is present, suggesting that the cellular $\mathrm{AT}_{1}$ receptor/AT ${ }_{2}$ receptor ratio determines the outcome (Schorb et al., 1993; Crawford et al., 1994; van Kesteren et al., 1997). Increasing $\mathrm{AT}_{2}$ receptor expression in neonatal cardiomyocyte cultures mediates myocyte hypertrophy independent of AngII (D'Amore et al., 2005), indicating that the $\mathrm{AT}_{2}$ receptor is prohypertrophic. Studies of $\mathrm{AT}_{2}$ receptor knockout and transgenic mice are ambiguous on the physiologic role of the $\mathrm{AT}_{2}$ receptor in fibrosis. The myocardial perivascular fibrosis is increased in $\mathrm{AT}_{2}$ receptor null mice (Wu et al., 2002), and an opposite outcome was observed in a different $\mathrm{AT}_{2}$ receptor knockout mouse model (Kurisu et al., 2003). Overexpression of the $\mathrm{AT}_{2}$ receptor in the heart significantly inhibited AngII-induced increases in perivascular fibrosis (Kurisu et al., 2003) in one model, whereas in another mouse model interstitial collagen increased (Yan et al., 2003b).

With regards to hypertrophy of myocardium, $\mathrm{AT}_{2}$ receptor manipulation experiments in vivo yielded conflicting results. $\mathrm{AT}_{2}$ receptor knockout prevents the induction of hypertrophy upon AngII infusion (Ichihara et al., 2001). However, transgenic mice overexpressing the $\mathrm{AT}_{2}$ receptor in cardiomyocytes are normal and after AngII infusion they develop the same degree of hypertrophy as normal controls (Masaki et al., 1998; Moore et al., 2001; Yan et al., 2003b). Surprisingly the hypertrophic response to pressure overload is suppressed in $\mathrm{AT}_{2}$ receptor-null mice, suggesting the $\mathrm{AT}_{2}$ receptor is essential for hypertrophic process (Senbonmatsu et al., 2000). In a ventricular myocyte directed $\mathrm{AT}_{2}$ receptor overexpression model, the $\mathrm{AT}_{2}$ transgenic mice have an impaired $\mathrm{Ca}^{2+}$-dependent inotropic response to AngII, associated with reduced activity of the $\mathrm{Na}^{+} / \mathrm{H}^{+}$exchange (Nakayama et al., 2005). Transgene copy number influenced cardiac hypertrophy in this model. Hypertrophy under basal conditions was observed in MLC2v$\mathrm{AT}_{2} \mathrm{TG}$ mice with 18 copies of transgene with highest level of $\mathrm{AT}_{2}$ receptor expression, whereas mice with 9 transgene copies did not display any signs of cardiac hypertrophy or HF (Yan et al., 2003b). Subjecting the 9 transgene copy mice to pressure overload by aortic banding significantly reduced left ventricular myocyte diameter, systolic pressure, and collagen compared with aortic-banded nontransgenic controls (Yan et al., 2003b). Thus, the role played by $\mathrm{AT}_{2}$ receptor in heart is essential for both AngII-induced and pressure-overload myocardial hypertrophy, again raising doubts about the "yin-yang" paradigm that this receptor is a physiologic antagonist of $\mathrm{AT}_{1}$ receptor actions. 
3. Regulation of Fibrosis Response in Other Tissue. In vivo, PD123319 increases renal fibrosis, supporting the notion that the $\mathrm{AT}_{2}$ receptor is anti-fibrotic in kidney as well. However, there are also conflicting reports that chronic PD123319 administration reduces collagen content in rats (Levy et al., 1996). Aggravated renal injury is observed in $\mathrm{AT}_{2}$ receptor-knockout mice (Benndorf et al., 2009). The $\mathrm{AT}_{2}$ receptor-deficient mice had exaggerated mortality accompanied with increased risk of albuminuria, renal fibrosis, glomerular injury, lymphocyte infiltration, and chemokine expression compared with renal ablation control mice. The blood pressure and RAS metabolites were similar in these groups.

Studies in disease models carrying the $\mathrm{AT}_{2}$ receptornull genotype show greater susceptibility for pathogenesis of cerebral infarction and atherosclerosis (Iwai et al., 2005). Angiotensin $\mathrm{AT}_{2}$ receptor protects against cerebral ischemia induced neuronal injury (Li et al., 2005; Kaschina et al., 2008). In cerulean-induced pancreatitis, expression of $\mathrm{AT}_{2}$ receptor is associated with pancreatic fibrosis, which is increased through a TGF- $\beta-$ mediated mechanism (Ulmasov et al., 2009).

In the last 10 years, strides have been made toward understanding the role of the $\mathrm{AT}_{2}$ receptor in various pathogenic models (Knowle et al., 2000; De Paolis et al., 2002; Miura and Karnik, 2002). The $\mathrm{AT}_{2}$ receptor has been implicated in cardiovascular disease as a beneficial moiety due to its proposed vasodilatory role, ability to inhibit cell growth, induce apoptosis, and inhibit activation of MAPK functions that, overall, appear to counterbalance the actions of the $\mathrm{AT}_{1}$ receptor (Berk, 2003; Miura et al., 2010). Overall, the $\mathrm{AT}_{2}$ receptor may play a role in several $\mathrm{AT}_{1}$ receptor-independent biologic processes (Mifune et al., 2000; Ruiz-Ortega et al., 2000, 2001a; Zhu et al., 2000; Weidekamm et al., 2002; Benndorf et al., 2003; Gingras et al., 2003; Caballero et al., 2004; Zhao et al., 2005b; Mertens et al., 2010). Furthermore, activation of the $\mathrm{AT}_{2}$ receptor inhibits autophagy mediated by the $\mathrm{AT}_{1}$ receptor in cardiomyocytes (Porrello et al., 2009). Notably, activation of the $\mathrm{AT}_{2}$ receptor in cells of neuronal origin, induced neurite outgrowth and elongation, mediated cellular excitability, and migration and, in some cases caused, neuronal cell death (Guimond and Gallo-Payet, 2012). Taken together, it has become clear that the $\mathrm{AT}_{2}$ receptor signaling mechanisms are diverse and require further studies to fully elucidate the role of this receptor in biologic processes.

\section{H. Drug Targeting the AngII Type 2 Receptor}

Wan et al. (2004) developed a highly selective, orally accessible nonpeptide $\mathrm{AT}_{2}$ receptor agonist, called Compound 21 (C21). A potential therapeutic role for the $\mathrm{AT}_{2}$ receptor is being propagated since the discovery of $\mathrm{C} 21$; it can confer acute vasorelaxation in vitro and has been shown to have organ protection effects in vivo. Specifically, C21 was observed to improve postmyocardial infarction (Kaschina et al., 2008) and provide vasodepressor effects in spontaneously hypertensive rats (Bosnyak et al., 2010). In addition, the ligand was shown to reduce myocardial fibrosis and vascular injury in hypertensive stroke-prone rats (Rehman et al., 2012). Controversy surrounds the use of C21 for therapeutic intervention, however. It was recently speculated whether the effects of $\mathrm{C} 21$ were $\mathrm{AT}_{2}$ receptor specific or just off-target effects as others have cautioned (Guimond and Gallo-Payet, 2012; Verdonk et al., 2012). Therefore, development of additional $\mathrm{AT}_{2}$ receptor-specific agonists and antagonists is crucial and will expand the field, supporting an important role for targeting of the $\mathrm{AT}_{2}$ receptor in disease states.

The increased knowledge of the expression pattern and mechanisms of action of the $\mathrm{AT}_{2}$ receptor has substantially contributed to understanding the role of AngII in physiologic and pathologic conditions. Synthesis of additional highly selective $\mathrm{AT}_{2}$ receptor ligands will allow for the exploration of novel receptor-dependent effects in the cardiovascular, renal, and nervous systems. The ability of the $\mathrm{AT}_{2}$ receptor to be a therapeutic target is promising and provides insight into the complexities of RAS.

\section{Angiotensin II Type 3 Receptor}

In addition to $\mathrm{AT}_{1}$ receptor and $\mathrm{AT}_{2}$ receptor, the presence of an $\mathrm{AT}_{3}$ subtype was reported by Chaki and Inagami (Chaki and Inagami, 1992a; Inagami et al., 1993). Unique pharmacological properties observed in mouse neuroblastoma cells (Neuro-2A) led to the designation of new subtype, $\mathrm{AT}_{3}$ receptor. Binding affinity of this subtype is $\sim 12.0 \mathrm{nM}$ for $\left[{ }^{125} \mathrm{I}\right]$ AngII, which was inhibited by AngII with a $K_{\mathrm{i}}$ value of $\sim 7 \mathrm{nM}$. $\left[{ }^{125} \mathrm{I}\right] \mathrm{AngII}$ binding to this subtype was not inhibited by AngIII, the $\mathrm{AT}_{1}$ receptor antagonist Dup753 or the $\mathrm{AT}_{2}$ receptor antagonist PD123319. $\left[{ }^{125} \mathrm{I}\right]$ AngII binding was in sensitive to GTP analogs. This subtype expressed in differentiated Neuro-2A cells was shown to stimulate cGMP formation in these cells. The stimulation was blocked by the $\left[\mathrm{Sar}^{1}{ }^{\text {,Ile }}{ }^{8}\right.$ ]AngII but not by Dup753 or PD 123319 (Chaki and Inagami, 1992b). A 2.2-kb fulllength cDNA encoding a putative $\mathrm{AT}_{3}$ receptor was cloned by screening an adrenal cortex library (Inagami et al., 1993). The putative $\mathrm{AT}_{3}$ receptor cDNA encodes a $40-\mathrm{kDa}$ protein with $95 \%$ amino acid identity to $\mathrm{rAT}_{1}$ receptor. The overall nucleotide similarity is $71 \%$ because of low homology in the $5^{\prime}(58 \%)$ - and $3^{\prime}(62 \%)$ untranslated regions. A different receptor subtype, $\mathrm{rAT}_{1 \mathrm{C}}$, was reported to be $95 \%$ homologous to the $\mathrm{rAT}_{3}$ receptor nucleotide sequence and less so to $\mathrm{rAT}_{1 \mathrm{~A}} \mathrm{R}$ $(90 \%)$ and $\mathrm{rAT}_{1} \mathrm{R}(82 \%)$ subtypes. Expressed $\mathrm{AT}_{3}$ receptor in COS-7 cells mediates agonist-induced calcium mobilization that is not inhibited by $\mathrm{AT}_{1}$ receptor inhibitors. The $\mathrm{AT}_{3}$ receptor mRNA is reported to be most abundant in the adrenal cortex and pituitary and differs from $\mathrm{AT}_{1}$ 
receptor mRNA tissue distribution. The $\mathrm{AT}_{3}$ receptor sequence contains two additional potential phosphorylation sites for protein kinase C (Sandberg et al., 1992). Despite these initial efforts, further characterization of putative $\mathrm{AT}_{3}$ receptor has stalled. Furthermore, a gene for encoding the putative $\mathrm{AT}_{3}$ receptor is not annotated despite the availability of a cDNA clone, possibly suggesting further uncertainty for an $\mathrm{AT}_{3}$ receptor .

\section{Angiotensin IV Binding Site(s)}

Defined as novel "non-AT $\mathrm{A}_{1}$ and non-AT $\mathrm{T}_{2}$ ", angiotensin IV (AngIV: VYIHPF) binding entity associated with neuronal cognitive processes and renal vascular functions (Fig. 8) was initially nominated as the $\mathrm{AT}_{4}$ receptor in 1995 (de Gasparo et al., 2000). A specific membrane-binding site for the $\left[{ }^{125} \mathrm{I}\right]$ AngIV peptide was defined as the AngIV binding site (Swanson et al., 1992). This AngIV binding site was found to be concentrated in brain, heart, kidney, adrenals, and blood vessels (Wright et al., 1993, 2008). Functional distinction of AngIV binding to the AngIV binding site was demonstrated. AngIV analogs induced several marked biologic effects in central nervous, renal, pulmonary, and vascular tissues (Handa, 2001; Li et al., 2002; Vinh et al., 2008; Wright et al., 2008). Human LNPEP gene (Lnpep in rodents) product IRAP/AngIV binding site was identified and is believed to be the sole AngIV binding site until recently. Nearly 20 years of research only shows the complexity associated with the concept of a unique AngIV binding site in vivo that prevent unambiguously defining a receptor molecule that satisfactorily accounts for pharmacology and function as the AngIV receptor (the IUPHAR Nomenclature Committee, Karnik et al., 2014; http://www.guidetopharmacology.org/ GRAC/FamilyIntroductionForward?familyId=6.).

\section{A. Functional Definition of AngIV Binding Sites}

Existence of a heat resistant receptor that reversibly bound $\left[{ }^{125} \mathrm{I}\right]$ AngIV with high affinity $\left(K_{\mathrm{d}} \approx 1-5 \mathrm{nM}\right)$ in bovine adrenal membranes was described (Harding et al., 1992; Jarvis et al., 1992; Swanson et al., 1992; Sardinia et al., 1993). This receptor did not bind the peptide analogs of AngII, [Sar ${ }^{1}$ ] AngII, $\left[\mathrm{Sar}^{1}, \mathrm{Ile}^{8}\right.$ ] AngII, $\left[\mathrm{Sar}^{1}, \mathrm{Ala}^{8}\right]$ AngII, Ang(1-7), AngIII, and the nonpeptide inhibitors of $\mathrm{AT}_{1}$ and $\mathrm{AT}_{2}$ receptor Dup753 (losartan), PD123177, and CGP42112A (Harding et al., 1992; Swanson et al., 1992; Hanesworth et al., 1993). Histoautoradiographic mapping techniques determined the greatest concentrations of $\left[{ }^{125} \mathrm{I}\right]$ AngIV specific binding sites in the brain. The brain distribution of AngIV binding sites was distinct from that of both $\mathrm{AT}_{1}$ and $\mathrm{AT}_{2}$ receptors. These criteria lead to the term AngIV binding site, which is by definition, linked to regulation of cognitive, sensory, and motor functions. These AngIV binding sites are not associated with functions where $\mathrm{AT}_{1}$ receptor or $\mathrm{AT}_{2}$ receptor play a predominant role in physiologic functions, such as water-electrolyte balance, cardiovascular regulation, and control of thirst behaviors. However, AngIV effects observed in the cardiovascular and renal tissue, which was initially thought to be mediated by the AngIV binding site, may actually be mediated through the $\mathrm{AT}_{1}$ receptor. Therefore, definition of AngIV binding site excludes functions associated both $\mathrm{AT}_{1}$ and $\mathrm{AT}_{2}$ receptors.

\section{B. Structure of the AngIV Binding Protein, Insulin Regulated Amino Peptidase}

The putative AngIV binding site was biochemically identified as a $\sim 160$ - and $\sim 190-\mathrm{kDa}$ glycoprotein on reducing SDS-polyacrylamide gel (Bernier et al., 1995a; Wright and Harding, 1995). The adrenal and bovine endothelial AngIV binding sites were reported as dimeric on nonreducing gels at $225 \mathrm{kDa}$ (Bernier et al., 1998). Similarly, the AngIV binding site polypeptides from bovine heart, thymus, kidney, bladder, aorta, and hippocampus were dimers (Zhang et al., 1999). Soon after its characterization, AngIV binding site was thought not to be a GPCR because of its mobility on SDS gels as well as lack of GTP $\gamma$ S effect on $\left[{ }^{125} \mathrm{I}\right] \mathrm{AngIV}$ binding in rabbit heart, guinea pig brain, and rat vascular smooth muscle (Hall et al., 1993). That AngIV binding site is not a GPCR now seems to be unanimous.

By purifying and sequencing the putative AngIV binding protein, Albiston et al. (2001) identified it as insulin regulated aminopeptidase (IRAP, E.C. 3.4.11.3 also called LNEP for leucyl- $N$-exopeptidase). IRAP is a Type 2 transmembrane protein of the gluzincin aminopeptidase family (Rogi et al., 1996; Nomura et al., 2005). This family includes homologs such as aminopeptidases A and N (AP-N: endothelial cell 3.4.11.2). Reflecting distinct activities, the mammalian IRAP/AngIV binding site has many names, oxytocinase, cystinyl aminopeptidase, placental leucine aminopeptidase, gp-160, or vp165, depending on its independent cloning (Vauquelin et al., 2002a; Albiston et al., 2004a; Nomura et al., 2005; Tsujimoto and Hattori, 2005). Human IRAP/AngIV binding site contains 1025 residues with N-terminal 110-amino acid-long intracellular domain, a 21-residuelong transmembrane segment (111-131), and an 871residue-long extracellular C-terminal domain (132-1025), which contains the catalytic and zinc binding sites. The catalytic site is composed of a GXMEN motif that is crucial for the exopeptidase activity and includes the $\mathrm{Zn}^{2+}$-binding motif HEXXH-X $\mathrm{X}_{18}-\mathrm{E}$ (Kandror and Pilch, 1994; Kandror et al., 1994; Keller et al., 1995; Ross et al., 1996). HEK 293 T cells transfected with IRAP expression plasmid specifically bind the AngIV binding site ligands, AngIV and LVV-hemorphin 7, and compete for the binding of ${ }^{125} \mathrm{I}-\left[\mathrm{Nle}^{1}\right]$ AngIV with $\mathrm{IC}_{50}$ values of 32 and $140 \mathrm{~nm}$, respectively. Both immunohistochemistry and mRNA hybridization histochemistry analysis determined in parallel on the brain matched that of the AngIV binding site determined by radioligand binding. 


\section{IRAP/AngIV binding site}

Gene and Protein: The gene for IRAP/AngIV binding site is LNPEP (EC 3.4.11, chromosome 5, gene ID: 4012). It is a single pass 1025 residue long Type II trans-membrane zinc protease. It is also known as insulin-regulated aminopeptidase (IRAP), Vasopressinase, placental leucine aminopeptidase (PLAP), oxytocinase (Otase), cystinyl aminopeptidase (CAP).

\section{Functional Surrogates:}

1. Aminopeptidase N (AP-N, EC3.4.11.2) a paralog of LNPEP involved in processing of peptide hormones, such as angiotensin III and neuropeptides and may act as a receptor. AP-N (chromosome 15, gene ID 290) is also known as CD13; LAP1; P150; GP150. The 967 amino acid protein with long extracellular carboxyl terminal domain contains a consensus zincbinding sequence.

2. cMET/HGFR, is the proto-oncogene MET product hepatocyte growth factor receptor (EC 2.7.10.1, chromosome 7, gene ID 4233) is a receptor tyrosine-kinase, also known as HGFR; AUTS9; RCCP2; c-Met. Ligand Binding induces autophosphorylation that provides docking sites for downstream signaling molecules such as PI3-kinase or the adapter proteins leading to the activation of signaling cascades including the RAS-ERK, PI3 kinase-AKT, RAS-ERK pathways.

Tissue Distribution: Placenta, Uterus, Ovary, Brain, Smooth muscle, Heart, Kidney,Liver, Lung, skeletal muscle, Skin, Prostate, White blood cells and platelet.

Natural Ligands: Angiotensin 3-8 (AngIV), Vasopressin, Oxytocin, LVV-hemorphin, LVVhemorhin-7.

Agonists: Norleucine1-angiotensin VI, Norleucine1-Leu3-psi(CH2-NH2)3-4-angiotensin IV , Lys-Bradykinin.

Antagonists/Inhibitors: WSU 3033, Divalinal-AngIV (Divalinal), Compound 8 [beta(3)homotyrosine Ang IV], Compound 12 [beta(3)-homotyrosine Ang IV], Compound 7 [macrocyclic Ang IV], Aminopeptidase inhibitor 7B, Compound 19 [macrocyclic Ang IV] Ang IV-NH(2), IVDE77, AL-11.

Functional Assays: Memory retention, retrieval and reversal of induced-amnesia; Calcium Signaling; Enhanced synaptic long term potentiation (LTP) in dentate gyrus; Inhibition of neurohormone cleavage; Cerebral vascular relaxation and microcirculation; Ang IV dependent pulmonary endothelial NOS activation; Dopamine release in striatum.

Signaling: Intracellular calcium, PI3-kinase signaling; eNOS activation, MAPK activation, engage Tankyrase 1 and 2, Rab GTPase-activating protein As160.The signal transduction of $\mathrm{AT}_{4} \mathrm{R}$ can be questioned since it is not unequivocally shown to be mediated by the binding of AngIV to IRAP. Recombinant expression of human IRAP in cell lines failed to establish ligand induced signaling.

Physiological Consequences of Altering Gene Expression: The lnpep -null mice maintained normal glucose uptake and distribution of Glut4 into muscle and fat cells.

The loss of spatial memory and AngIV binding site are age related in lnpep-null mice. AngIVdependent fibrinolysis via PAI-1 and arterial thrombosis after vascular injury is attenuated in the lnpep -null mice. The lnpep -null mice are less sensitive to the development of acute pentylenetetrazol (PTZ)-induced seizures. The lnpep -null mice lack antidepressant-effect of oxytocin, less risk for ischemic brain damage and stroke analogous to the effect of Ang IV.

Fig. 8. IRAP/AngIV binding site. See main text for details and Supplemental Material.

The AngIV binding site ligands dose dependently inhibit the IRAP catalytic activity. In vivo, IRAP hydrolyzes the N-terminal amino acid from neuropeptides including arginine-vasopressin, cholecystokinin-8, dynorphin, met-enkephalin, lysine-bradykinin, neurokinin A, neuromedin B, oxytocin and somatastatin, (Herbst et al., 1997; Matsumoto et al., 2001; Lew et al., 2003). Accordingly, IRAP was proposed as the first AngIV binding site (see Fig. 8 and Supplemental Material).

Albiston et al. (2001) proposed that the AngIV binding site ligands may exert their effects by inhibiting the catalytic activity of IRAP and extending the halflife of its neuropeptide substrates to potentiate cognitive functions attributed to AngIV binding to the AngIV binding site. The LNPEP gene product (Lnpep gene in rodents) was considered the sole AngIV binding site until recently (Vauquelin et al., 2002a).

\section{Pharmacology of AngIV Analogs}

1. Agonists of the AngIV Binding Site. The characteristics of the AngIV molecule critical for high-affinity binding to the AngIV binding site in tissue include preservation of residues at positions 1,2 , or 3 of the AngIV, whereas positions 4, 5, and 6 can be replaced (Sardinia et al., 1993; Wright et al., 1995). A detailed study (see Supplemental Material) of position 1 of AngIV 
revealed that high-affinity AngIV binding requires a primary amine in position 1 and L-hydrophobic residues at position 1 favor binding. Thus, L-norleucine1AngIV resulted in a very high-affinity analog $\left(K_{\mathrm{d}}\right.$ in the picomolar range). The $\mathrm{CH}_{2}-\mathrm{NH}$ isostere substituted at 1-2 peptide linkage produced a high-affinity analog that increases rotation of peptide bond (Sardinia et al., 1993). An L-aromatic amino acid at position 2 and L-hydrophobic amino acid at position 3 of the AngIV molecule are critical to bind to AngIV binding site. Deletion of the C-terminal phenylalanine from of AngIV does not alter binding affinity, but this derivative is inactive in manipulating physiologic blood flow (Wright et al., 1995). The minimal AngIV-like peptide ligand to bind AngIV binding site with high affinity requires a sequence, $\mathrm{Val}^{1}-\mathrm{Tyr}^{2}-\mathrm{Ile}^{3}-\mathrm{R} 1-\mathrm{R} 2$. An agonist requires a phenylalanine in addition at the $\mathrm{C}$-terminal position, a structural feature of AngIV, which would also permit activation of signaling through the ubiquitously present $\mathrm{AT}_{1}$ receptor. In most studies of AngIV analogs, inadvertent activation of the $\mathrm{AT}_{1}$ receptor may have to be carefully ruled out. The peptide LVV-hemorphin-7 binds and activates the AngIV binding sites in vivo. LVV-H7 specifically displaced $\left[{ }^{125} \mathrm{I}\right]$ AngIV binding to adrenal and brain membranes.

2. Antagonists of the AngIV Binding Site. Harding's laboratory made early strides overcoming the difficulties of design and synthesis of AngIV binding site antagonists. They had success with Val substitution coupled with reducing peptide bonds in the Val-TyrVal segment generating divalinal-AngIV (Sardinia et al., 1993; Sardinia et al., 1994; Krebs et al., 1996). A second antagonist with norleucine in position 1 , leucine in position 3, and reduced peptide bonds at the first and third bond positions (Norleual-AngIV) was subsequently created and characterized. This analog binds the AngIV binding site with high affinity and has been reported to not bind $\mathrm{AT}_{1}$ receptor and $\mathrm{AT}_{2}$ receptor. Divalinal-AngIV prevented c-Fos expression, blood flow induced by AngIV in brain, as well as the inhibition of proximal tubule sodium transport by AngIV (Handa et al., 1998).

3. Inhibitors of the Insulin Regulated Amino Peptidase Activity. Concept of IRAP as the potential AngIV binding site stimulated development of newer and more selective analogs and resulted in the development of a new class of cognitive enhancers, which target the catalytic site of IRAP (Albiston et al., 2008) (see Supplemental Material). Efforts were taken to develop AngIVderived IRAP inhibitors with improved selectivity versus $\mathrm{AP}-\mathrm{N}$ and the $\mathrm{AT}_{1}$ receptor, as well as resistance to proteolytic degradation. Lukaszuk et al., 2008) reported that the AngIV analog AL-11 ( $\mathrm{p} K_{\mathrm{i}} 7.25$ for IRAP enzyme activity) in which the $\beta$-homo amino acid $\beta^{2} \mathrm{hVal}$ is responsible for stability and the $\beta^{3}$ hPhe for selectivity. Further improvement was obtained by substitution of $\mathrm{His}^{4}-\mathrm{Pro}^{5}$ with a constrained Trp analog (Aia-Gly) to obtain AL-40 with a $K_{\mathrm{i}}$ is 8.07 for IRAP (Lukaszuk et al., 2009). Substitution of the latter Gly ${ }^{5}$ by norvaline led to an analog (IVDE77) with further increased affinity $\left(\mathrm{p} K_{\mathrm{i}}\right.$ is 8.77 for IRAP) (Lukaszuk et al., 2011; Nikolaou et al., 2013). The latter compound was also ${ }^{3} \mathrm{H}$-labeled and characterized as a useful tool for the detection of IRAP/AngIV binding site under physiologic conditions, i.e., in the absence of any metal ion chelators (Nikolaou et al., 2013).

Synthesis of peptidomimetics targeting IRAP activity lead to successful replacement of the C-terminal His-Pro-Phe in AngIV replaced by small aromatic $\gamma$-turn mimetics (Compound 29, $K_{\mathrm{i}}=26 \mathrm{nM}$ ). Compounds with low nanomolar inhibitory activity and enhanced selectivity for IRAP over the structurally related amino peptidase, AP-N, were obtained. Additional studies are needed to elucidate bioactive conformation for IRAP inhibition and presumably functional selectivity for AngIV binding site-mediated cognitive effects (Anderson et al., 2010, 2011; Axen et al., 2006). With a bioinformatics in silico screening approach, a large library of compounds against a structure homology of IRAP (Ye et al., 2008; Albiston et al., 2011) benzopyran compounds were identified, synthesized, and optimized; the highest affinity compound HFI- 437 has a $\mathrm{p} K_{\mathrm{i}}$ of 7.70 for IRAP. The latter nonpeptide inhibitors were found to enhance the performance of rats in different memory paradigms (Mountford et al., 2014).

Yang et al. (2008) used IRAP/AngIV binding siteselective ligands, LVV-hemorphin-7 and AT4-16, for intravenous, intrarenal, and intracerebroventricular infusion to discriminate AngIV effects through $\mathrm{AT}_{1}$ and AngIV binding sites. They observed that AngIV had no effects on mean arterial pressure, renal blood flow, or urinary sodium excretion in the presence of IRAP inhibitors. In addition, they reported that the majority of the AngIV-sensitive aminopeptidase activity in kidney membranes is due to AP-N and not the IRAP/AngIV binding site. Some of the central and peripheral vasoactive effects of AngIV were mediated through the AngIV binding sites in other studies (Lew et al., 2003; Axen et al., 2007). Increase in blood pressure from chronic AngIV elevation in the brain was found to be $\mathrm{AT}_{1}$ dependent in a transgenic mouse model, although specificity of action of AngIV in this model should be a question (Lochard et al., 2004). Thus, central and peripheral pressor and vasoconstrictor effects of AngIV are very likely mediated through $\mathrm{AT}_{1}$ receptor in vasculature. The activation of the $\mathrm{AT}_{1}$ receptor by AngIV is a well established pharmacological effect, but this effect is distinct from the memory-enhancing effects of AngIV (Yang et al., 2008). Stragier B. et al. (2006) independently demonstrated that AngIV and LVV-H7 did not significantly alter hippocampal blood flow as determined by laser Doppler flow measurement, excluding the vascular effects responsible for cognitive functions of either peptide (Jewett et al., 1991) (see Fig. 8 and Supplemental Material). 


\section{Signaling}

1. Effects on Insulin Regulated Amino Peptidase Signaling as a Transmembrane Receptor. Prior to the identification of IRAP as AngIV binding site, the AngIV binding site was thought to be a multimeric, $225-\mathrm{kDa}$ complex in bovine tissues (Bernier et al., 1995b; Zhang et al., 1999). It is now clear that AngIV binding sites are not coupled to G-proteins in tissues such as bovine endothelium, guinea pig brain, and rat vascular smooth muscle (Hall et al., 1992). The N-terminal domain of IRAP may participate in signal transduction with AngIV acting as an agonist in the activation of intracellular signaling, which may mediate the physiologic effects of AngIV. Vauquelin et al. (2002b) proposed that IRAP is a classic transmembrane receptor, with an intracellular tail that would interact with intracellular signaling molecules. This idea is based on the finding that AngIV interacts with a juxta-membrane domain of IRAP/AngIV binding site in bovine heart membranes. This putative binding site for AngIV is different from that of the active site of IRAP, which implies that AngIV binding may modulate transmembrane signal transduction by AngIV binding site and the IRAP activity by an allosteric mechanism (Caron et al., 2003). In the structurally related AP-N, binding of monoclonal antibodies activates IP3linked increase of intracellular calcium and phosphorylation of MAPK (Navarrete Santos et al., 2000a,b,c). Expression of c-Fos is induced by intracerebroventricular infusion of AngIV in brain centers of rats, which are associated with cognition (Roberts et al., 1995). Several studies have shown that AngIV binding site ligands are capable of activating different intracellular signaling pathways. These include a variety of mechanism(s), including an increase of the intracellular calcium concentration, modulation of MAPK activation of $\mathrm{NF}_{\kappa} \mathrm{B}$ signaling, increased cGMP production, and more downstream changes in DNA synthesis. It should also be noted that in no classic signaling could be demonstrated in $\mathrm{CHO}$ cells despite the presence of AngIV binding sites (Albiston et al., 2007). In pulmonary artery endothelial cells, AngIV binding to AngIV binding sites activates eNOS and stimulates cGMP accumulation and causes pulmonary arterial vasodilation (Patel et al., 1998). This effect was blocked by divalinal AngIV as well as by the phosphoinositide 3-kinase inhibitors (Patel et al., 1998). Induction of PAI-1 by AngIV has been implicated in the pathogenesis of renal interstitial fibrosis in several forms of chronic glomerulonephritides (Gesualdo et al., 1999). AngIV stimulates cell proliferation in rat anterior pituitary without requiring $\mathrm{AT}_{1}$ receptor (Ptasinska-Wnuk et al., 2003) and also activates $\mathrm{NF} \kappa \mathrm{B}$, leading to the transcription of proinflammatory genes, intercellular adhesion molecule-1, IL-6, MCP-1, PAI-1, and TNF $\alpha$ in VSMCs. These effects were blocked by treatment with AngIV binding site antagonist, suggesting that signal transduction activated by
AngIV may underlie pathogenesis of cardiovascular diseases (Esteban et al., 2005).

IRAP inhibitors, such as divalinal-AngIV and LVVhemorphin-7, which bind to the IRAP active site directly, produce similar effects. For instance, increasing the extracellular dopamine levels in the striatum of the rat by these inhibitors was comparable to AngIV. This finding suggests that active-site binding of the inhibitors mediates modulation of extracellular dopamine levels in striatum (Stragier et al., 2004). In a related study, the authors hypothesized that IRAP and/or AP-N possibly act as receptors for IRAP inhibitors and AngIV in mediating dopamine release in the striatum by receptor-mediated signaling (Stragier et al., 2007). High-affinity binding of AngIV to only the Zn-depleted IRAP apoenzyme may inhibit IRAP activity by stabilizing an inactive apoenzyme. AngIV binding to the catalytic domain of IRAP has been clearly established in vitro, and the evidence for AngIV binding to an allosteric site in the juxtamembrane region has been questioned. $\left[{ }^{125} \mathrm{I}\right] \mathrm{AngIV}$ binds in an a 1:1 ratio to in the presence and absence of the juxtamembrane region, suggesting that there is only one AngIV binding site but the site is not located in the juxtamembrane region. AngIV binding to the apoenzyme can be measured in vitro, reflecting possibly a different mechanism of binding and stabilization of AngIV when $\mathrm{Zn}$ is removed. Taken together, AngIV appears to bind better to the IRAP apoenzyme than the native IRAP. AngIV binding to native AP-N or to the AP-N apoenzyme is poor (Demaegdt et al., 2006), indicating that AP-N as an in vivo receptor for AngIV is poorly supported by data. Thus, redundancy of mechanism of AngIV receptors is possible.

2. Effects on Insulin Regulated Amino PeptidaseDependent Neuropeptide Potentiation. On the basis of the kinetics of inhibition, AngIV and its analogs have been proposed as in vivo inhibitors of neuropeptide catabolism by IRAP (Ye et al., 2007). AngIV and divalinal display competitive kinetics, indicating that AngIV binding site ligands mediate their peptidase inhibitor effects by binding to the catalytic site of IRAP (Lew et al., 2003). IRAP is known to play a role in the in vivo metabolism of vasopressin and Lys-bradykinin bound to the enzyme (Herbst et al., 1997; Wallis et al., 2007). Substrates identified in vitro for IRAP include vasopressin (AVP), oxytocin, somatostatin, Leu- and Metenkephalin, and Lys-bradykinin (Herbst et al., 1997; Matsumoto et al., 2001; Lew et al., 2003). These neuropeptides enhance memory consolidation and retrieval (Vauquelin et al., 2002b; Wallis et al., 2007). The physiologic effects of AngIV binding site ligands therefore may result from the inhibition of cleavage of neuropeptides by IRAP altering memory processing (Lew et al., 2003). Accumulation of neuropeptides when AngIV binds to IRAP has been suggested as a mechanism for cognitive enhancement. In a recent study it 
was shown that the increase of extracellular dopamine concentration and the anticonvulsant effect of AngIV could be inhibited by a somatostatin receptor antagonist administered in the rat striatum (Li et al., 2009a). Accumulation of somatostatin to mediate these effects may be one possible mechanism for the observed phenomenon, although somatostatin cleavage by IRAP was not confirmed. At the moment there is no direct in vivo evidence that supports the IRAP/AngIV binding-mediated neuropeptide potentiation hypothesis.

3. AngIV Effects on IRAP Trafficking. Although AngIV and its analogs are not cleaved by IRAP, they competitively inhibit the in vitro catalytic activity of IRAP. On this basis, it has been suggested that IRAP inhibitor binding may prolong the cell surface localization of IRAP/AngIV binding sites in neurons to potentiate cognitive and memory functions (Heimfeld et al., 1992; Demaegdt et al., 2004a,b). In tissues not directly involved in cognitive functions, insulin and some IRAP substrates peptide hormones have been shown to regulate kinetics of cell-surface trafficking of IRAP. For instance, in vascular endothelial cells in placenta, intracellular vesicle-bound IRAP responds to oxytocin by translocating the IRAP vesicles to the plasma membrane. By catabolizing oxytocin and vasopressin, translocated intracellular vesicle-bound IRAP regulates oxytocin and vasopressin levels in serum during pregnancy and thereby prevents early contractions and premature delivery. Cotranslocation of IRAP and the glucose transporter GLUT4 associated vesicles to the plasma membrane in adipocytes is stimulated by insulin (Demaegdt et al., 2011). The cytosolic N-terminal domain of IRAP binds $\mathrm{p} 115$, a protein that mediates transport vesicle fusion and is cotranslocated with IRAP and GLUT4 to the plasma membrane (Hosaka et al., 2005). The intracellular domain of IRAP plays a role in regulating GLUT4 vesicle trafficking through interaction with specific proteins including AS160 (Larance et al., 2005; Peck et al., 2006; Albiston et al., 2007). GLUT4 has a critical role in insulin-stimulated glucose uptake in these cells (Keller et al., 1995; Ross et al., 1996). The subcellular localization of IRAP in neurons demonstrated striking parallels with distribution of IRAP in insulin responsive adipocytes and muscle cells, where the enzyme plays a role in insulin-regulated glucose uptake. Therefore, the function of IRAP in neurons may be similar to that in insulin responsive cells (Fernando et al., 2007). Relevant to cognitive function of IRAP/AngIV binding site in brain, the majority of IRAP was localized in intracellular vesicles in mouse brain neurons (Fernando et al., 2005, 2007). The binding of IRAP inhibitors may prolong the cell surface localization of IRAP/AngIV binding site and GLUT4 in neurons. The resulting increase of glucose uptake may be responsible for IRAP ligands to facilitate memory in hippocampus-dependent memory deficits in models for amnesia (Fernando et al., 2007; Stragier et al., 2008).
The IRAP inhibitors enhance cAMP-stimulated or depolarization-evoked glucose uptake mediated by GLUT4 (Fernando et al., 2008). Although both AngIV and LVV$\mathrm{H} 7$ potentiate glucose uptake into cultured hippocampal neurons in culture, these effects were not confirmed in vivo. Extracellular hippocampal glucose levels significantly decreased while the animals explored the maze. When the animals were not in a maze, glucose levels in their hippocampus remained constant. However, the same analysis performed in the plus maze test showed that both AngIV and LVV-H7 did not significantly alter hippocampal glucose levels compared with control. Increased glucose availability for hippocampal neurons as the basis of enhanced memory seems unlikely.

\section{E. Distribution of the of AngIV Binding Sites}

Consistent with the functional definition, the $\left[{ }^{125} \mathrm{I}\right]$ AngIV autoradiographic mapping demonstrated that highest densities of the putative AngIV binding site are localized in brain regions associated with cognition and sensomotor functions (Moeller et al., 1996). The brain distributions of the AngIV binding sites mapped are confirmed for cross-species consistency in rat, guinea pig, rhesus monkey (Miller-Wing et al., 1993; Roberts et al., 1995; Wright et al., 1995; Moeller et al., 1996), and human brain slices (Moeller et al., 1996; Chai et al., 2000). Binding of $\left[{ }^{125} \mathrm{I}\right] \mathrm{AngIV}$ was reported in brain structures associated with motor functions (Moeller et al., 1995, 1996; Wright and Harding, 1995), in the forebrain (Miller-Wing et al., 1993; Moeller et al., 1995, 1996), in the cerebellum (Miller-Wing et al., 1993), and in the primary motor neocortex (Moeller et al., 1996). The brain autonomic nuclei, dorsal motor nucleus of the vagus, nucleus ambiguus, rostral ventral lateral medulla, and paraventricular nucleus of the hypothalamus are sites of high AngIV binding site density (Moeller et al., 1995, 1996). Lower density of AngIV binding sites exists in sensory structures and primary sensory neocortex (Miller-Wing et al., 1993; Moeller et al., 1995, 1996). It is also well known that AngIV is generated from AngII or AngIII and present in the circulation (Semple et al., 1976; Abhold and Harding, 1988). Independent studies demonstrated that a hemoglobin $\beta$-chain fragment isolated from sheep brain with a sequence, LeuVal-Val-hemorphin-7 (LVV-H7) is also capable of binding and activating the in vivo physiologic effects attributed to activation of putative AngIV binding sites (Moeller et al., 1997). The distribution of ${ }^{125}$ I-LVV-H7 binding sites in brain slices was identical to those for $\left[{ }^{125} \mathrm{I}\right] \mathrm{AngIV}$, suggesting that it is an endogenous AngIV binding site ligand (Moeller et al., 1997, 1998; Lee et al., 2001, 2003).

Distribution of $\left[{ }^{125} \mathrm{I}\right]$ AngIV binding sites have been documented in different species for peripheral tissues, including bovine adrenal cortex $\left(B_{\max } \approx 4 \mathrm{pmol} / \mathrm{mg}\right)$ (Harding et al., 1994); monkey and rat kidney $\left(B_{\max } \approx\right.$ $1.0 \mathrm{pmol} / \mathrm{mg}$ ) (Harding et al., 1994), rat, guinea pig, and rabbit hearts $\left(B_{\max } \approx 0.3-0.8 \mathrm{pmol} / \mathrm{mg}\right)$; and guinea pig 
and bovine vascular smooth muscle ( $\left.B_{\max } \approx 1.0 \mathrm{pmol} / \mathrm{mg}\right)$. Mapping the binding of AngIV in rat kidney demonstrated high density of AngIV binding site in the outer stripe of the medulla, the glomeruli, and the medullary core. Surprisingly the AngIV binding map is distinctly different from AngIV binding site distribution map in kidney regions (Harding et al., 1994; Coleman et al., 1998). More recently AngIV binding site binding sites were described in mouse macrophages. The observations that the transcription of the IRAP gene in these cells was upregulated in proinflammatory M1-activated macrophages and the cell surface AngIV binding site binding is modulated upon exposure to IFN- $\gamma$, LPS, or exogenous particles suggest a possible function of AngIV binding sites in these cells (Nikolaou et al., 2014).

Although the distribution of AngIV is not known in central and peripheral tissue location of AngIV binding sites, the ubiquitous presence of amino peptidases, AP-A and AP-N, can locally produce AngIV from AngII. Both these enzymes have been localized to the plasma membrane of pericytes, suggesting that AngIV can be produced in the extracellular space surrounding microvessels in the brain (Healy and Wilk, 1993; Kunz et al., 1994). Several reports indicate that, within neurons, nearly $80 \%$ of AngII is converted to AngIV (Stragier et al., 2008). Exogenous administration of AngIV has been shown to increase cerebral microcirculation (Naveri et al., 1994; Kramar et al., 1997; Kramar et al., 1998; Lanckmans et al., 2007a,b). In terms of function, intracranially administered AngIV and its functional analogs facilitate memory in rodent behavior models (Braszko et al., 1988; Wright et al., 1993; Tchekalarova et al., 2001; Lee et al., 2003). AngIV also facilitates reversal of memory shortfalls produced by scopolamine, mecamylamine, abusive alcohol dose, ischemia, as well as by disruption of the perforant path in the hippocampus (Borawska et al., 1989; Wright et al., 1996; Pederson et al., 1998; Albiston et al., 2004a; Olson et al., 2004). AngIV enhances neuronal long-term potentiation in vitro and in vivo (Kramar et al., 2001; Wayner et al., 2001) as well as attenuates PTZinduced seizures (Tchekalarova et al., 2001; Stragier et al., 2006) and protects against cerebral ischemia (Faure et al., 2006a,b, 2008). It is reported that in peripheral tissue AngIV administration increases renal blood flow that was blocked by antagonist divalinal-AngIV (Coleman et al., 1998; Hamilton et al., 2001). This effect was also accompanied with an increased urinary sodium excretion (Hamilton et al., 2001). Contrasting results have been reported indicating that AngIV decreased renal blood flow (Gardiner et al., 1993; Fitzgerald et al., 1999; Yang et al., 2008) and increased blood pressure (Yang et al., 2008). The latter effects were prevented by $\mathrm{AT}_{1}$ receptor antagonists.

\section{F. Insulin Regulated Amino Peptidase Gene} Knockout Mice

1. Evaluation of AngIV-dependent Functions. Considering the wide-ranging effects mediated by IRAP and its inhibitors, understanding the genetic basis of IRAP functions is critical. In normal and memorycompromised rodent models, enhancement of memory upon intracerebroventricular delivery of AngIV and its analog peptides is the defining pharmacological property, which is thought to be mediated solely by the IRAP/AngIV binding site (Albiston et al., 2004b; Lee et al., 2004). Biochemically, these ligands show highaffinity binding to the IRAP catalytic site. Therefore, the inhibition of IRAP activity or genetic loss of expression would be expected to improve memory.

Albiston et al. (2010) evaluated effects of IRAP gene knockout on AngIV binding and the behavioral phenotype in mice. In the IRAP-null mouse, the complete loss of IRAP expression in muscle, fat, and brain including hippocampus was accompanied with loss of binding sites for the radioligand $\left[{ }^{125} \mathrm{I}\right] \mathrm{Nle} 1-\mathrm{AngIV}$. However, the IRAP-null genotype did not significantly affect the glucose transporter GLUT4 levels in the hippocampus and cortex. There was no detectable difference in the response to depolarization-evoked glucose uptake in the hippocampal slices of the IRAP-null mice compared with their wild-type littermates. Comprehensive neurologic testing of adult IRAP-null mice confirmed that the sensory perception, their motor reflexes, and vestibular function were normal. The performance of young knockout mice in memory and behavioral testing paradigms was similar to the wild-type mice. In the IRAPnull mice, the sensory, locomotor, and anxiety responses were not altered at 3-4 months of age. Furthermore, the IRAP knockout mice demonstrated improved cognitive and spatial memory when tested at 6 and 12 months of age on the Y maze task. A limited impact of IRAP-null genotype in enhancing memory was unexpected, questioning the basis of linking IRAP-inhibition to memory and cognitive enhancement.

The unexpected phenotype could be the result of developmental alteration due to germ-line deletion of the IRAP gene causing susceptibility to age-related cognitive decline. Several questions regarding IRAP-null mice remain unanswered. For instance, whether cognitive enhancement from AngIV and analogs of AngIV is completely abolished in the IRAP-null mice. The authors have demonstrated that IRAP-null mice show complete loss of AngIV binding using tissue imaging methods; they did not examine whether central infusion of AngIV or other IRAP inhibitors had any functional effect on improving performance in a range of memory paradigms or whether a compensatory mechanism is activated as a stand-in for the loss of IRAP in specific neurons. Wallis et al. (2007) reported increased levels of plasma AVP in the IRAP-null mouse, 
suggesting that IRAP may play a role in regulating levels of vasopressin. However, it was difficult to reconcile IRAP-/- phenotype with increased AVP and rather extensive literature on AVP effects on different brain regions. Does IRAP play a role in only disease paradigms while an unknown molecule functions as the mediator of physiologic effects of AngIV? Loyens et al. (2011) attempted to examine this question in a recent report, demonstrating that IRAP-null mice show decreased susceptibility to pentylenetetrazol (PTZ)-induced seizures. They observed that thresholds for myoclonic twitch and generalized clonic seizures significantly increased in $\operatorname{IRAP}(-/-)$ mice compared with their IRAP(+/+) littermates when $\mathrm{PTZ}$ is injected demonstrating that that $\operatorname{IRAP}(-/-)$ mice are less sensitive to the development of PTZ-induced seizures. The authors concluded that IRAP is involved in generalized seizure generation in normal mice. Pham et al. (2012) demonstrated that deletion of the IRAP gene protects the brain from ischemic damage analogous to the effect of the IRAP inhibitor AngIV. Transient middle cerebral artery occlusion in mice produces stroke-induced neurologic deficits 24 hours after reperfusion in mice. The infarct volumes measured were significantly reduced in the IRAP knockout mice compared with wild-type littermates, with corresponding improvement in neurologic performance. Cerebral blood flow was increased in the IRAP knockout animals.

2. Nuances for Defining Receptor(s) for AngIV. More than a decade of research focused on delineating the IRAP/AngIV binding site as the sole AngIV binding site has exposed some pharmacological, signaling, and genetic criteria not satisfactorily met by this protein. Other binding proteins have been proposed as alternative to the concept of IRAP/AngIV binding site for AngIV (De Bundel et al., 2008; Stragier et al., 2008; Wright et al., 2008).

AP-N, another transmembrane peptidase structurally related to IRAP/AngIV binding site was suggested as an additional candidate AngIV receptor, because this protein can activate IP3-dependent intracellular calcium and MAPK signaling (Navarrete Santos et al., $2000 \mathrm{~b}, \mathrm{c})$. However, evidence demonstrating dimerization of AP-N and signaling in response to AngIV binding is lacking. More importantly, the role of this protein in AngIV-mediated cognitive enhancement is currently not established.

The ability of AngIV analogs to alter cognitive function and augment neurite outgrowth in vitro led to the hypothesis that the c-Met tyrosine kinase receptor for the hepatocyte growth factor (HGF) is a candidate AngIV binding site (Wright et al., 2008). Sequence-based prediction identified the possibility for AngIV and its analogs to disrupt HGF interaction with c-Met. As predicted, in picomolar concentration Norleual-AngIV was found to displace HGF binding to c-Met and antagonize signaling activity of HGF through c-Met in vitro. Norleual-AngIV blocks HGF-induced proliferation, invasion, cell scattering, and wound closure in vitro; inhibits ex vivo angiogenesis; and attenuates melanoma lung colonization in vivo. Some of the HGF/c-Met functions partially overlap with classic functions attributed to the AngIV binding site, including facilitated memory consolidation and hippocampal LTP and calcium signaling. These studies suggest that the c-Met is a cellular receptor candidate for mediating biologic effects of picomolar concentrations of AngIV analogs. These authors suggested MSP/Ron receptor system as an additional potential target of AngIV analogs that warrants investigation (Wagh et al., 2008). A number of questions regarding c-Met as a potential AngIV binding site remain unexplored. For instance, can AngIV specifically activate and AngIV-antagonists inhibit the c-Met receptor signaling in vivo to reproduce functions attributed to classic AngIV/AngIV binding site system? Does the distribution of c-Met receptor in brain and its levels within cognitive centers correlate with the classic mapped AngIV binding sites? Does LVV-H7 and the other IRAP-selective ligands discussed above bind to the c-Met receptor? Does recombinant c-Met show high-affinity binding to AngIV and LVV-H7?

3. AngIV Binding Site Conclusion. The state of IRAP/AngIV binding site protein as the sole receptor for mediating in vivo functions of AngIV will have to be revisited in future. Multiple lines of evidences suggest that IRAP/AngIV binding site protein is a high-affinity AngIV binding receptor that mediates some of the neuroprotective and cognitive effects of AngIV, although the exact signaling mechanisms mediating these effects have not been clearly established. The IRAP/AngIV binding site hypothesis has stimulated development of rather potent and specific inhibitors of its enzymatic function, which have been shown to be cognitive enhancers in vivo. At present existing data indicate that IRAP/AngIV binding site protein does not discriminate between agonist and antagonist ligands and does not directly account for transmembrane signaling. Better characterization of IRAP/AngIV binding site inhibitors using a broad range of assays may unravel intricacies of AngIV functions in vivo. Future developments in this field are likely to uncover additional AngIV receptors and mechanisms of AngIV action in vivo. Such receptors will have to address several aspects of AngIV physiology, for example: i) in vivo efficacy at picomolar and subnanomolar concentrations at which AngIV and its analogs were found to affect changes in physiologic function; ii) the rapid onset of the physiologic effects of AngIV in blood flow and intracellular calcium signaling or neuronal potentiation experiments; iii) pharmacological effects of the "agonists" and "antagonists" on the receptor-specific effects on downstream signaling; and iv) global genetic deletion of receptor abolishes pharmacological and physiologic effects of AngIV and increases 
susceptibility to neurodegeneration or decline of cognitive functions. This is critical because the AngIV/ AngIV binding site system is implicated in cognitive, memory, and cerebroprotective functions, as well as disease conditions like Alzheimer's, seizures, and Parkinson's disease. The lack of AngIV binding site agonists and antagonists penetrating blood-brain barrier has limited our understanding of importance in the etiology and treatment of memory dysfunctions associated with dementia and degenerative diseases.

\section{Receptor Conundrum in the Renin-Angiotensin System}

Based on the multiple criteria used to recommend the pairing of an orphan receptor with its cognate ligand, MAS was included among the "orphan" GPCRs, for which the status of endogenous ligand(s) remains unclear (Davenport et al., 2013). The status of MAS is unchanged in the current update to IUPHAR/BPS Guide to Pharmacology. MAS is a candidate receptor for endogenously produced RAS peptide hormone Ang(1-7) (Tirupula et al., 2014, http://www.guidetopharmacology.org/GRAC/ ObjectDisplayForward?objectId=150.).

MAS was initially discovered in 1986 as an oncogene that transformed NIH3T3 cells and induced tumors in nude mice (Young et al., 1986). The amino acid sequence of MAS bears fingerprint of GPCRs, and tumorigenic activity of overexpressed receptor was confirmed later (Janssen et al., 1988; van 't Veer et al., 1993). However, the genomic Mas as an oncogene is disputed, because this gene is not amplified in tumors. Overexpression of MAS, however, can transform cells. MAS was originally proposed as a functional receptor for AngII that also mediated response to angiotensins I and III in Xenopus oocytes (Jackson et al., 1988). In transfected cells, MAS mediated intracellular calcium mobilization and DNA synthesis responses to AngII and III (Jackson et al., 1988; Jackson and Hanley, 1989; McGillis et al., 1989; Dean and Boynton, 1990). However, the status of MAS as AngII receptor was disproved. The cell lines where AngII and MAS interactions were characterized expressed endogenous $\mathrm{AT}_{1}$ receptor (Ambroz et al., 1991; Monnot et al., 1991; Murphy et al., 1991; Sasaki et al., 1991), thus ending the controversy of AngII activating MAS (Sasamura et al., 1992).

\section{A. Pairing MAS Receptor with Ang (1-7)}

Current peer review literature supports pairing of MAS with multiple endogenous peptides with preference toward studies involving Ang(1-7). Originally discovered as a GPCR with transforming activity (1986) and the founding member of the Mas-related G-proteincoupled receptor subfamily of neurohormone receptors (2001), MAS remained orphan until the neuropeptide $\mathrm{FF}$ was shown to activate G-protein signaling through this receptor (see Fig. 9). Two years later,
MAS-transfected cells were shown to produce arachidonic acid response to Ang(1-7) (Santos et al., 2003b; Gembardt et al., 2008; Zimpelmann and Burns, 2009). The angiotensin-converting enzyme 2 metabolizes AngI and AngII to Ang(1-7) (IUPHAR-DB ID no. 582 ), which functions as a vasodilator and antiproliferative agent. Some of the actions of Ang(1-7) appear to be mediated via MAS in vivo (Santos et al., 2003b). Deletion of the Mas gene in mice abolishes the binding of Ang(1-7) in mouse kidney as well as abolishes the antidiuretic action of the peptide after an acute water load (Santos et al., 2003b). Many in vivo effects attributed to Ang(1-7) are diminished or absent in Mas-null mouse tissues and organs, providing support for the proposed ligand receptor pairing (Walther et al., 1998; da Costa Goncalves et al., 2007; Fraga-Silva et al., 2008; Santos et al., 2008; Pinheiro et al., 2009; Mario et al., 2012). An evolutionary account of MAS and related GPCRs is presented in an extensive review recently by Bader et al. (2014). Hence, further input from the scientific community is needed to validate MAS as the cognate receptor for Ang(1-7).

Alamandine is a relatively new heptapeptide that contains an Ala replacing Asp at position 1 of Ang(1-7). This peptide can be generated by either decarboxylation of the Asp residue of angiotensin-(1-7) or by catalytic action of angiotensin-converting enzyme-2 on decarboxylated AngII (also called angiotensin A) (Lautner et al., 2013). Two primary journal articles on alamandine, since its discovery, have paired this ligand with Masrelated G-protein-coupled receptor D; however, neither strong pharmacological nor direct radioligand binding data exist (Habiyakare et al., 2014; Wilson et al., 2015). Identification of alamandine and its putative receptor has attracted attention as a novel mediator of physiologic and pathophysiological actions of the RAS and may help to develop new therapeutic strategies for treating human cardiovascular diseases.

Conventional G-protein signaling by MAS, reported to be stimulated by the physiologic ligand neuropeptide FF (Dong et al., 2001), was recently confirmed (Tirupula et al., 2014). Reports pairing MAS with several other physiologic and synthetic peptides exist, such as with angiotensin III, angiotensin IV, angioprotectin P33/ CGEN-857, P61/CGEN-856, and MBP-7 (Bikkavilli et al., 2006; Gembardt et al., 2008; Shemesh et al., 2008; Savergnini et al., 2010; Jankowski et al., 2011). Consequently, these other physiologic peptides reported as endogenous ligands for MAS remain incompletely characterized. Few reports indicate that G-proteins are activated by MAS in response to few physiologic peptides and "surrogate" nonpeptide ligands in transfected cells but not in response to Ang(1-7) (Bikkavilli et al., 2006; Canals et al., 2006; Shemesh et al., 2008; Tirupula et al., 2014; Zhang et al., 2012). MAS shares significant similarity with Mas-related G-protein-coupled receptors or paralogs, which are predominantly expressed in 

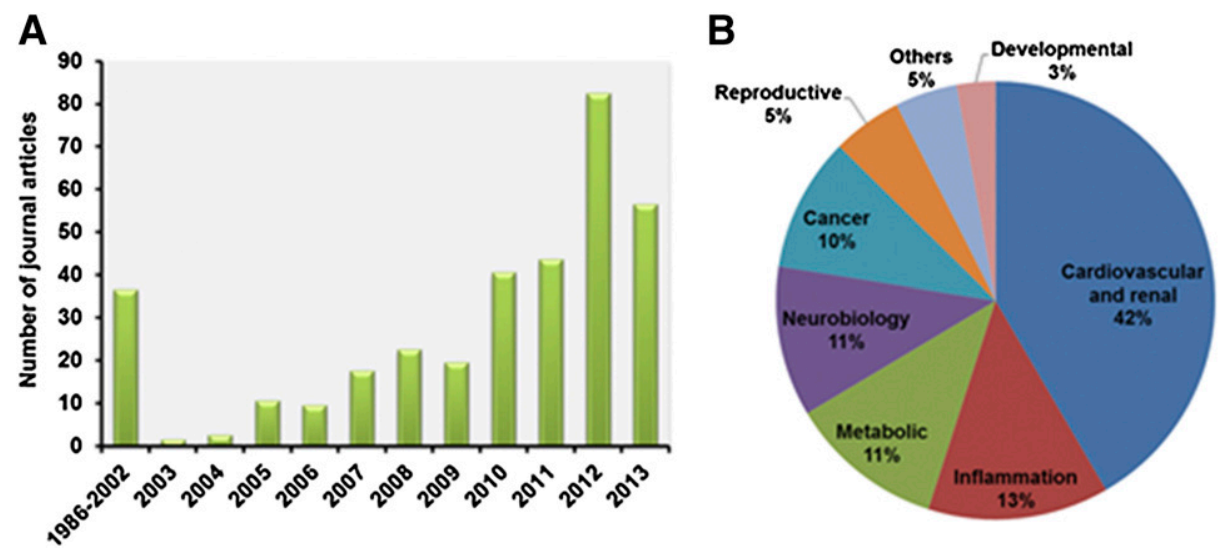

Fig. 9. Literature search and analysis of primary journal articles on MAS. The journal articles published on MAS were automatically retrieved from PubMed with the search term "((((MAS[Title/Abstract]) AND angiotensin OR MAS1[Title/Abstract]) AND angiotensin[Title/Abstract]) NOT meconium aspiration syndrome[Title/Abstract]) NOT medication adherence scale[Title/Abstract])". The results were manually curated to add or remove relevant or irrelevant references, respectively, and the primary journal articles were separated from the reviews, interviews, lectures, or commentary articles. The final curated list had a total of 337 primary journal articles until the end of year 2013. These articles are further analyzed to illustrate (A) number of publications per year as a bar graph and (B) percentage of publications per functional category as a pie chart. The functional categorization was done by searching for key words in the abstract and the title representing a given category. Please note that in the functional categorization, some articles are present in multiple functional categories and therefore $100 \%$ represents a higher total of articles (524) instead of 337. The figure helps to illustrate the general point that the number of publications on MAS have dramatically increased post-2003 and that MAS literature is primarily representative of the cardiovascular and renal function category. Python and Biopython scripts were used to aid in the literature search and analysis.

neurons and immune cells (Young et al., 1986; Dong et al., 2001; Bender et al., 2002). MAS might be expected to respond to physiologic ligands, which are typically neuropeptides and pro- or anti-inflammatory ligands (Dong et al., 2001; Lembo et al., 2002; Choi and Lahn, 2003; Tatemoto et al., 2006; Liu et al., 2009; Kashem et al., 2011; Subramanian et al., 2011; Han et al., 2013). Therefore, the role of MAS as the receptor for only Ang(1-7) qualifying it as an integral constituent of the RAS is unclear. MAS appears to be a GPCR with high constitutive activity and may not have a unique cognate endogenous ligand (Canals et al., 2006; Zhang et al., 2012; Tirupula et al., 2014). Many physiologic modulators of MAS activity may exist; important among them may include peptides such as Ang(1-7) and neuropeptide FF. Only when a clear understanding of the physiology, pharmacology, and pathology for all ligands of MAS has begun to emerge, can the status of MAS be assigned with greater certainty. The current objective is to stimulate research into confirming multiple ligands pairing with MAS in physiologic regulations. A brief perspective on MAS given below summarizes its potential relationship to RAS and pathophysiological regulation in vivo.

\section{B. Pharmacology}

Using Mas-knockout mice in studies, Santos et al. (2003b) first proposed that Ang(1-7) is the physiologic ligand for MAS, a discovery that has transformed research on MAS (see Figs. 9 and 10). The basis for initial proposal of Ang(1-7) as an endogenous MAS ligand is the observed loss of Ang(1-7) tissue effects in the MAS-null mice. Proposed pairing of Ang(1-7) with MAS was further confirmed in receptor binding and functional studies in MAS-transfected cells. Specific $\left[{ }^{125} \mathrm{I}\right]$ Ang(1-7) binding to kidney sections was reduced in MAS-null mice (Santos et al., 2003b; Pinheiro et al., 2004) as well as in MAS-GFP transfected cells (Gironacci et al., 2011). The Ang(1-7) antagonist A-779 competed with an $\mathrm{IC}_{50}$ close to the $K_{\mathrm{d}}$ of ${ }^{125} \mathrm{I}-\mathrm{Ang}(1-7)$ in Mastransfected cells (Santos et al., 2003b; Gironacci et al., 2011). Binding specificity to MAS was reported by using fluorescently labeled Ang(1-7) in CHO cells (Pinheiro et al., 2004; Savergnini et al., 2010; Jankowski et al., 2011), platelets (Fraga-Silva et al., 2008), and Leydig cells (Leal et al., 2009). Specific binding of fluorescently labeled Ang(1-7) was absent in MAS-null mice and the binding is blocked by A-779 and AVE0991, a nonpeptide analog of Ang(1-7) (Wiemer et al., 2002). However, in most of the published reports on MAS, standard pharmacological binding experiments are absent, presumably due to technical problems. Overall, radiolabeled or fluorescently labeled Ang(1-7) data in most reports intended to confirm a direct interaction between Ang(1-7) and MAS are of poor pharmacological rigor. This is a serious setback in the deorphanizing endeavor.

\section{Signaling}

Ang(1-7) treatment causes concentration-dependent release of arachidonic acid by stimulating mitogenactivated protein kinase p38 in human mesangial cells and MAS-transfected CHO and COS cells (Santos et al., 2003b; Gembardt et al., 2008; Zimpelmann and Burns, 2009). Ligand treatment with Ang(1-7) or AVE0991 in MAS-transfected cells couples calcium-independent activation of nitric oxide synthase to the phosphatidylinositol 3-kinase/protein kinase B, AKT pathway (Sampaio 


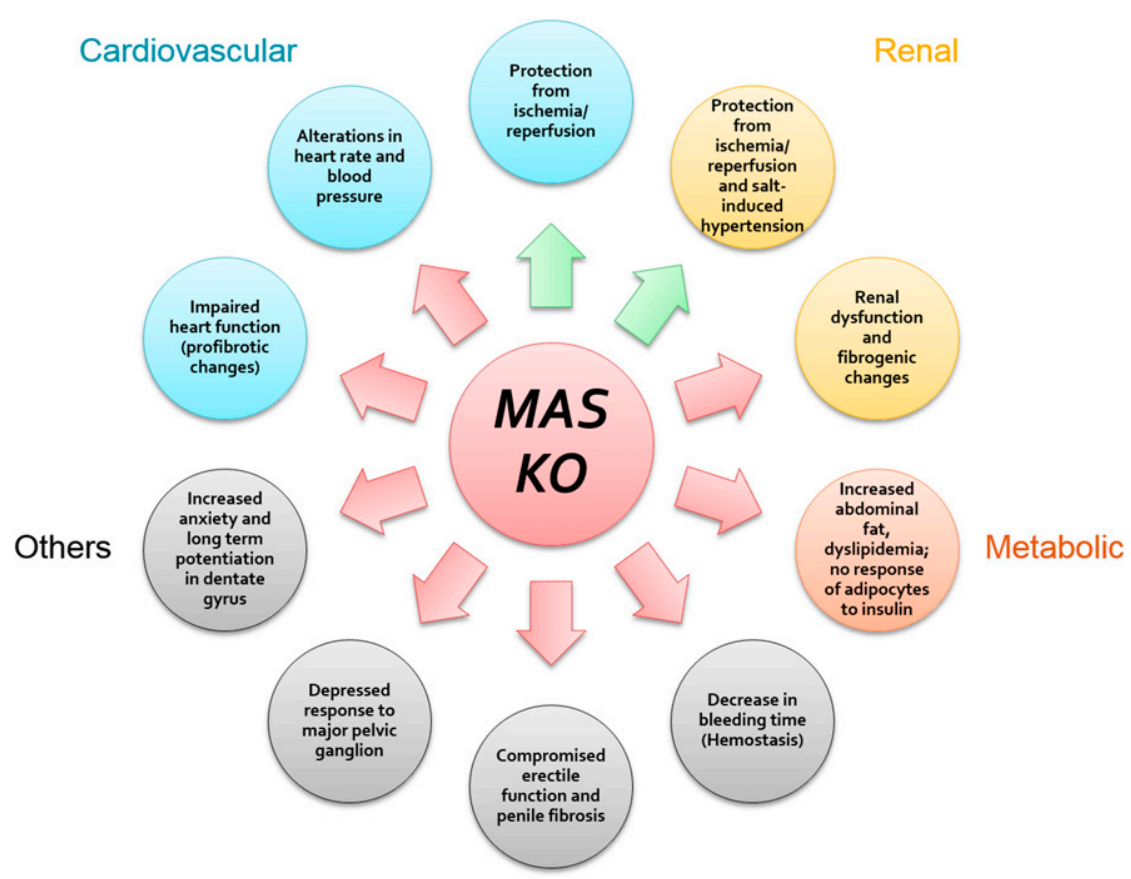

Fig. 10. Summary of phenotypes reported in MAS knockout mice. The figure summarizes multiple phenotypes that are reported in literature in mice lacking the MAS gene. Both damaging (red arrows) and protecting (green) arrows are observed in knockout mice. See main text for references.

et al., 2007b; Lopez Verrilli et al., 2012; Savergnini et al., 2013; Than et al., 2013), or activation of phospholipase A2 (Santos et al., 2003b). Ang(1-7) altered the phosphorylation of MAP kinases (Sampaio et al., 2007a; Zimpelmann and Burns, 2009; Liu et al., 2012; Verano-Braga et al., 2012). Thus, Ang(1-7) action through MAS is proposed to be production of arachidonic acid and activation of nitric oxide synthase, which may not involve cAMP, IP3, and calcium signaling.

Although MAS is a GPCR, there is no evidence for Ang(1-7)-mediated conventional G-protein signaling as measured by the levels of second messenger molecules like calcium, IP3, and cAMP in MAS-transfected cells (Bikkavilli et al., 2006; Shemesh et al., 2008; Zhang et al., 2012; Tirupula et al., 2014). This observation is, however, disputed by reports in kidney, where Ang(1-7) treatment is reported to increased cAMP levels and protein kinase A activation, suggesting $\mathrm{G} \alpha_{\mathrm{s}}$ activation by MAS. These signals could be inhibited using A-779 (Magaldi et al., 2003; Liu et al., 2012). MAS was shown to constitutively couple to $\mathrm{G} \alpha_{\mathrm{i}}, \mathrm{G} \alpha_{\mathrm{q}}$, and $\mathrm{G} \alpha_{12 / 13}$ proteins (Zohn et al., 1998; Whitehead et al., 2001; Booden et al., 2002; Chen and Ikeda, 2004; Singh et al., 2010a; Tirupula et al., 2014). Adenovirus-mediated overexpression of MAS in rat neonatal cardiomyocytes caused IP3 accumulation and myocyte hypertrophy through $\mathrm{G} \alpha_{\mathrm{q}}$-mediated signaling (Zhang et al., 2012). Constitutive activity of the MAS in overexpressing cells activates G-protein signaling pathways (Dias-Peixoto et al., 2008; Shemesh et al., 2008; Gomes et al., 2012; Zhang et al., 2012; Tirupula et al., 2014). Similar to Ang(1-7) the analogs A-779 and AVE 0991 fail to activate G-protein signaling through MAS (Fontes et al., 1994; Wiemer et al., 2002; Kluskens et al., 2009). Thus, the current state of Ang(1-7) interaction with MAS with regards to G-protein activation is controversial.

Conventional G-protein signaling by MAS was reported upon stimulation with another physiologic ligand, neuropeptide FF (Dong et al., 2001). Few other reports indicated that the G-protein activation by MAS was both constitutive and in response to some peptide and synthetic ligands (Bikkavilli et al., 2006; Canals et al., 2006; Shemesh et al., 2008; Zhang et al., 2012; Tirupula et al., 2014). In these studies, disparate ligands such as MBP7 (Bikkavilli et al., 2006), the angiotensin metabolite peptides AngIII and AngIV (Gembardt et al., 2008), novel nonpeptide ligands AR234960 (1-[[(4S)-4-(3-fluorophenyl)-1-(2-methoxy-4-nitrophenyl)sulfonylpyrrolidin-3-yl] methyl]-4-pyridin-2-ylpiperazine), AR244555 (1'-but3-enyl-5-chlorospiro[2H-indole-3,4'-piperidine]-1-yl)-(2,6difluorophenyl)methanone), and AR305352 (Zhang et al., 2012), and CGEN-856/P61 (Peptide sequence: FLGYCIYLNRKRRGDPAFKRRLRD) (Shemesh et al., 2008; Savergnini et al., 2010) were found to modulate MASmediated G-protein signaling pathways at relatively high concentrations of each peptide. Nonpeptide ligands with both agonist and inverse agonist properties and high selectivity for MAS are now known. The agonist AR234960 was shown to activate G-protein signaling and the inverse agonist AR244555 selectively inhibited the agonist response as well as the constitutive activity of MAS (Zhang et al., 2012; Tirupula et al., 2014). Remarkably, CGEN-856S is reported to activate calcium and AKT signals (Shemesh et al., 2008; Savergnini et al., 2013). Thus, atypical signaling response suggests functional selectivity of MAS, which allows 
interaction with different ligands to activate different signaling pathways, a phenomenon also called biased signaling (Tirupula et al., 2014).

\section{Other Receptors for the Proposed Ligands}

Other receptors such as the $\mathrm{AT}_{2}$ receptor can bind Ang(1-7) (De Souza et al., 2004; Walters et al., 2005). Similarly, NPFF is known to activate distinct GPCRs, NPFFR1 and 2. Thus, it is not clear whether the interaction between these ligands and MAS is mutually exclusive.

\section{E. Tissue Distribution of MAS}

The pattern of tissue distribution of MAS is broadly conserved in mammals. The highest expression of MAS is in brain and testis, but in other organs low level expression is detected (Metzger et al., 1995; Alenina et al., 2002). Mas gene is expressed in the hippocampus, cerebral cortex, the dentate gyrus, the olfactory tubercle, and the olfactory bulb in rodent brain (Young et al., 1988; Bunnemann et al., 1990); particularly interesting to note is that MAS expression is observed in regions of brain important for cardiovascular regulation (Becker et al., 2007). Mas gene expression was also discovered in a wide variety of rodent tissues (Villar and Pedersen, 1994; Metzger et al., 1995; Alenina et al., 2008). The MAS seen in the endothelial cells in blood vessels of different organs including heart is very low (Kumar et al., 1996; Alenina et al., 2008). MAS expression in the rat brain starts at postnatal day 1 . In adult rats, MAS level is transiently up regulated after episodes of seizure in the hippocampus (Martin et al., 1992; Martin and Hockfield, 1993). MAS expression starts after birth in testis and its levels increase during puberty (Metzger et al., 1995; Alenina et al., 2002). MAS was proposed to be a component of brain RAS in 1992, based on colocalization mapping of MAS, AngII, and AngII receptors with angiotensinogen, renin, angiotensin converting enzyme, and angiotensin fragments (Bunnemann et al., 1992).

\section{F. Mas Gene-Knockout in Mice.}

Availability of Mas knockout mice since 1998 provided unique opportunity to study the involvement of MAS in behavioral, cardiovascular, renal processes, and several metabolic physiologies (Walther et al., 1998). Multiple phenotypes are reported in literature for Mas knockout mice, both damaging and protective effects on organs and tissue as detailed in Fig. 10.

Mas knockout mice show increased anxiety and altered variability of heart rate and blood pressure in a sex-specific manner (Walther et al., 1998, 2000a,b). The early Mas knockout mice were in a mixed genetic background $(129 \times \mathrm{C} 57 \mathrm{BL} / 6)$ in which variability in pathophysiological consequences was observed. Significant alterations in cardiovascular and metabolic physiologies were evident only in knockout mice with pure genetic backgrounds like C57BL/6 and FVB/N (Xu et al., 2007; Santos et al., 2008). Mas knockout mice harbor impaired cardiac function accompanied with profibrotic expression of extracellular matrix proteins in the hearts (Santos et al., 2006; Gava et al., 2012). Renal dysfunctions associated with fibrotic changes were also reported (Pinheiro et al., 2009). The Mas knockout animals have increased expression of the proinflammatory cytokine, transforming growth factor- $\beta$, type 1 , and increased expression of profibrotic and proinflammatory molecules such as fibronectin, collagen, $\alpha$-smooth muscle actin, vimentin, connective tissue growth factor (CTGF), and tumor necrosis factor- $\alpha$ (Santos et al., 2008). Overexpression of MAS inhibited the expression of the proinflammatory genes. MAS-deficient mice have increased abdominal fat mass, dyslipidemia, increased levels of insulin and leptin, and altered response of adipocytes to insulin (Santos et al., 2008; Mario et al., 2012). Mas knockout offers protection from salt-induced hypertension (Heringer-Walther et al., 2012) and from ischemia/ reperfusion injury in both kidney and heart (Castro et al., 2006; Esteban et al., 2009; Zhang et al., 2012). In addition the Mas knockout, mice are also reported to have changes in hemostasis (Fraga-Silva et al., 2008) and penile fibrosis (da Costa Goncalves et al., 2007). A common mechanism behind the Mas knockout mice, exhibiting altered heart rate and blood pressure, increased vascular resistance, and decrease blood flow, is thought to be endothelial dysfunction due to imbalances in nitric oxide (NO) and reactive oxygen species (Castro et al., 2005; da Costa Goncalves et al., 2007; Peiro et al., 2007; Rabelo et al., 2008; Xu et al., 2008; Rakušan et al., 2010; Botelho-Santos et al., 2012).

\section{G. Pathophysiological Evidence of MAS Receptor Interaction with Ang(1-7).}

The vasodilation, antidiuresis, and antithrombosis responses to Ang(1-7) are lost in the Mas knockout mice (Santos et al., 2003b; Fraga-Silva et al., 2008). Validation of the cardiovascular actions of Ang(1-7) in the MAS-deficient mice take advantage of agonists AVE 0991 (Wiemer et al., 2002) and CGEN-856S (Shemesh et al., 2008) and the antagonists A-779 (Santos et al., 1994, 2003b) and D-Pro7 Ang(1-7) (Santos et al., 2003a). Based on the important functional evidences obtained in Mas knockout mice and the anti-inflammatory and antifibrotic effects of MAS in wild-type mice, MAS was proposed to be an important component of the RAS. Possibilities to enhance in vivo protective functions through Ang(1-7)-mediated activation of MAS make it an enticing drug target (Steckelings et al., 2011b). For example, inhibition of MAS function is shown to offer protection from ischemia/reperfusion injury in both mouse kidney and heart, making it a target receptor in the RAS for drug development (Castro et al., 2006; Esteban et al., 2009; Zhang et al., 2012). However, the physiologic effects of Ang(1-7) treatment combined with 
MAS deficiency in mice appear to be very complicated. For example, multifaceted effects of Ang(1-7) in vasculature may involve MAS interaction with $\mathrm{AT}_{1}$ receptor and $\mathrm{AT}_{2}$ receptor (Castro et al., 2005). MAS was shown to function as a physiologic antagonist of $\mathrm{AT}_{1}$ receptor by altering AngII response in the mice (Von Bohlen und Halbach et al., 2000; Kostenis et al., 2005; Rakušan et al., 2010). MAS-AT 1 receptor heterooligomerization resulted in the altered trafficking of $\mathrm{AT}_{1}$ receptor in transfected cells (Kostenis et al., 2005; Canals et al., 2006; Santos et al., 2007). Thus, the evidence for MAS interacting with $\mathrm{AT}_{1}$ receptor and $\mathrm{AT}_{2}$ receptor may be a mechanism for MAS in RAS. Literature regarding Ang (1-7) interaction with MAS in various organs is summarized below, and for a detailed literature compilation we refer to recent review by Bader et al. (2014).

1. Heart. Picomolar concentrations of Ang(1-7) were reported to cause a significant vasodilator effect in isolated rat hearts (Souza et al., 2013). Acute stimulation with Ang(1-7) in cardiomyocytes has no evident effect on calcium transients but stimulates release of NO (Dias-Peixoto et al., 2008; Costa et al., 2010; Gomes et al., 2010). However, an increased uptake and SERCA2 expression in transgenic rats overexpressing Ang(1-7) was reported by Gomes et al. (2012). Mas knockout alters expression of calcium handling proteins and decreased contractility of heart in mice (Santos et al., 2006; Botelho-Santos et al., 2012; Gava et al., 2012; Gomes et al., 2012). The cardiomyocytes in Mas knockout mice increase caveolin 3 expression and decrease HSP90, which reduces eNOS activity (Wu, 2002; Takahashi and Mendelsohn, 2003; Dias-Peixoto et al., 2008).

MAS RNA expression is reported in cardiomyocytes, cardiac fibroblasts, and the sinoatrial node cells (Ferreira et al., 2011). Ang(1-7) inhibits agonist-stimulated hypertrophy in culture cardiomyocytes (Gallagher et al., 2008; Gomes et al., 2010; Flores-Munoz et al., 2012), which can be blunted by siRNA-mediated knock down of MAS (Tallant et al., 2005). In transgenic rats, elevation of circulating Ang(1-7) causes attenuated cardiac fibrosis in response to isoproterenol (Santos et al., 2004b). In support of this finding, many investigators have evaluated the antiremodeling effects of Ang(1-7) and other MAS agonists, AVE 0991 and CGEN-856S (He et al., 2004; Iwata et al., 2005; Grobe and Katovich, 2006; Iusuf et al., 2008; Mercure et al., 2008; Giani et al., 2010; Gomes et al., 2010; Durik et al., 2012; Patel et al., 2012). Acute blockade of MAS with A-779 produces a deterioration of isolated mouse heart functions (Castro et al., 2005). Thus, independent studies support functional pairing of MAS and Ang(1-7) in myocardium; however, efficacy of this system needs rigorous proof.

2. Kidney. Although MAS expression could not be detected in the human kidney (Shalamanova et al., 2010), MAS transcripts have been detected in the kidney of rodents (Santos et al., 2003b; Pinheiro et al., 2004; da Silveira et al., 2010). Mas knockout mice lack the antidiuretic action of Ang(1-7) (Santos and Baracho, 1992). Both the anti- and proinflammatory effects of Ang(1-7) were reported in kidney of Mas knockout mice (Esteban et al., 2009; Singh et al., 2010b; Moon et al., 2011; Bernardi et al., 2012; Giani et al., 2012; Harris, 2012; Chou et al., 2013; Santos et al., 2013).

3. Vasculature. Studies performed in MAS models of gain- or loss-of-function show that systemic and local hemodynamics are chronically affected. In humans and other species, the endothelial layer of blood vessels produce Ang(1-7) (Santos and Baracho, 1992) and the presence of MAS in endothelial cells and VSMC is conserved, suggesting localized functional interaction (Santos et al., 2000; da Costa Goncalves et al., 2007; Sampaio et al., 2007b; Xu et al., 2008). Mas knockout increases the vascular resistance in mice in various organs (Botelho-Santos et al., 2012). In the transgenic rats, with increased circulating Ang(1-7), an Ang(1-7)dependent vasodilatory tone in blood vessels is suggested. Endothelial dysfunction is observed for Mas knockout in the FVB/N background, causing blood pressure increases (Xu et al., 2008). However, in C57Bl/6 mice, MAS knockout does not alter blood pressure (Rabelo et al., 2008). Short-term Ang(1-7) infusion in normotensive rats enhanced endothelial function (Faria-Silva et al., 2005), which may be also be conserved in other species (Langeveld et al., 2008; Durand et al., 2010; Stegbauer et al., 2011; Beyer et al., 2013; Jarajapu et al., 2013; Tassone et al., 2013).

4. Brain. Reduction in baroreflex sensitivity and sympathetic activity is observed in Mas-null mice (Walther et al., 2000b). In addition Ang(1-7) and MAS provide a protective effect in models of stroke (Mecca et al., 2011; Regenhardt et al., 2013, 2014a,b). MAS is present in select regions of the brain, which provides the structural basis for many effects produced by Ang(1-7) in the brain. For instance, the modulation of sympathetic activity (Silva et al., 1993; Fontes et al., 1994; da Silva et al., 2011; Kar et al., 2011; Li et al., 2013), increase of vagal tone (Guimaraes et al., 2012), and improvement of baroreflex sensitivity (Chaves et al., 2000) are Ang(1-7) responses, and these effects have been shown to be blocked by the MAS antagonist A-779.

5. Reproductive Organs. MAS is expressed in the testis, and changes in its expression are regulated during puberty (Metzger et al., 1995; Alenina et al., 2002). However, fertility is not affected in Mas knockout mice (Walther et al., 1998), but an increased risk of fibrosis and erectile dysfunction was reported (da Costa Goncalves et al., 2007). In humans, correlation between MAS expression in seminiferous tubules and male infertility was observed (Reis et al., 2010). MAS gene expression was reported in human ovaries (Reis et al., 2011). Both Ang(1-7) and MAS modulate folliculogenesis, ovulation, and pregnancy (Viana et al., 2011). 
Consistent with a regulatory role of MAS in ovary, the MAS antagonist A-779 was shown to prevent breakdown of germinal vesicle and reduced oocyte maturation induced by Ang(1-7) (Honorato-Sampaio et al., 2012). Expression of MAS in uterus and placenta is thought to be relevant to diseases such as preeclampsia (Velloso et al., 2007). Thus, MAS may not be important modulator of male and female fertility but may be relevant in pathogenesis related to fertility.

\section{H. Conclusion}

The current literature on MAS lacks convincing evidence for a direct interaction between MAS and Ang(1-7) with a potency that is consistent with a physiologic functions altered by Ang(1-7) and Mas knockout. Although independent groups have supported this pairing between MAS and Ang(1-7) in cellular functional and in vivo physiologic studies, caution expressed is based on basic pharmacological and mechanistic experiments, which are relevant and need to be addressed. The status of other possible ligands pairing with MAS and physiologic function for those ligands through MAS are important additional factors. Therefore, Ang(1-7)/ MAS pairing remains controversial and more research is required for NC-IUPHAR to make a formal assignment of MAS as the receptor for the RAS hormone Ang(1-7).

\section{Overall Summary}

The angiotensin receptor field has seen enormous development in the last 15 years on the structure, pharmacology, signaling, physiology, and disease states related to these receptors. The importance of each receptor in cardiovascular, hemodynamic, neurologic, renal, and endothelial functions, as well as in cell proliferation, survival, matrix-cell interaction, and inflammation, seems very well recognized. Therefore therapeutic agents targeted to these receptors are either in active use in clinical intervention of common diseases or under evaluation for repurposing in many other disorders.

Conclusions drawn earlier that the majority of known cardiovascular effects of AngII are mediated by the $\mathrm{AT}_{1}$ receptor is firmly established. Precision analysis of various facets of $\mathrm{AT}_{1}$ receptor mechanism has taken the place in explaining processes such as vasoconstriction, electrolyte-water balance, cellular proliferation and hypertrophy, and migration and extracellular matrix regulation. Precision analysis of function seems to be the new vogue in $\mathrm{AT}_{1}$ receptor research, resulting in efforts to dissect out a range of questions directed to cell-specific functions, ligandspecific signals, and inhibitor-specific therapeutic applications. Availability of three-dimensional structures for $\mathrm{AT}_{1}$ receptor bound to anti-hypertensive agents is expected to advance into new areas of $\mathrm{AT}_{1}$ receptor structure-function. New signaling modalities, such as functional selectivity of AngII analogs for enhancing $\beta$-arrestin-biased protective signaling, are now entering Phase II clinical studies for the treatment of acute $\mathrm{HF}$ and may do so to support vasculature and kidney function. $\mathrm{AT}_{1}$ receptor role in transactivation of receptor tyrosine kinases and mediating mechanical stress is gaining attention. Thus, position of $\mathrm{AT}_{1}$ receptor as a master regulator is well established.

The $\mathrm{AT}_{2}$ receptor as a functional antagonist of $\mathrm{AT}_{1}$ receptor has unraveled through studies of genetically altered mouse models, signal transduction analysis, and, most importantly, through development of new generation of ligands targeting this receptor. The literature on the $\mathrm{AT}_{2}$ receptor is quite extensive, demonstrating unconventional ligand selectivity, agonistindependent signaling, and cGMP/NO signaling by $\mathrm{AT}_{2}$ receptor. Identification of novel $\mathrm{AT}_{2}$ receptor-interacting proteins in recent years has rekindled interest in signaling mechanisms of this enigmatic receptor. Development of pharmacological agents targeted to $\mathrm{AT}_{2}$ receptor could find therapeutic application in protection of kidney, pain management, and stimulation of nerve growth.

The status of AngIV binding site and MAS as angiotensinergic mediators is still evolving. Functional studies clearly demonstrate important roles for these in central nervous system and in renal and cardiovascular regulation. Especially, MAS interaction with Ang(1-7) has gained significant attention. Multiple receptor candidates and multiple endogenous ligands for the AngIV binding site as well as for MAS may be physiologic. However, pharmacological characterization of these physiologic entities do not rise to the level of acceptance as specific angiotensin receptors at this time. Rigorous pharmacology and signaling studies are warranted.

\section{Acknowledgments}

This article was written as a result of updating the IUPHAR-BPS database by the Angiotensin Receptors Nomenclature Subcommittee for the International Union of Pharmacology.

\section{Authorship Contributions}

Wrote or contributed to the writing of the manuscript: Karnik, Unal, Kemp, Tirupula, Eguchi, Vanderheyden, Thomas.

\section{References}

Abadir PM, Periasamy A, Carey RM, and Siragy HM (2006) Angiotensin II type 2 receptor-bradykinin $\mathrm{B} 2$ receptor functional heterodimerization. Hypertension 48: 316-322.

Abbasi S, Su B, Kellems RE, Yang J, and Xia Y (2005) The essential role of MEKK3 signaling in angiotensin II-induced calcineurin/nuclear factor of activated T-cells activation. J Biol Chem 280:36737-36746.

AbdAlla S, Abdel-Baset A, Lother H, el Massiery A, and Quitterer U (2005) Mesangial AT1/B2 receptor heterodimers contribute to angiotensin II hyperresponsiveness in experimental hypertension. J Mol Neurosci 26:185-192.

AbdAlla S, Lother H, Abdel-tawab AM, and Quitterer U (2001a) The angiotensin II AT2 receptor is an AT1 receptor antagonist. J Biol Chem 276:39721-39726.

AbdAlla S, Lother H, el Massiery A, and Quitterer U (2001b) Increased AT(1) receptor heterodimers in preeclampsia mediate enhanced angiotensin II responsiveness. Nat Med 7:1003-1009.

AbdAlla S, Lother H, Langer A, el Faramawy Y, and Quitterer U (2004) Factor XIIIA transglutaminase crosslinks AT1 receptor dimers of monocytes at the onset of atherosclerosis. Cell 119:343-354. 
AbdAlla S, Lother H, and Quitterer U (2000) AT1-receptor heterodimers show enhanced G-protein activation and altered receptor sequestration. Nature 407: 94-98.

Abhold RH and Harding JW (1988) Metabolism of angiotensins II and III by membrane-bound peptidases from rat brain. J Pharmacol Exp Ther 245:171-177.

Abreu JG, Ketpura NI, Reversade B, and De Robertis EM (2002) Connective-tissue growth factor (CTGF) modulates cell signalling by BMP and TGF-beta. Nat Cell Biol 4:599-604.

Adachi Y, Saito Y, Kishimoto I, Harada M, Kuwahara K, Takahashi N, Kawakami R, Nakanishi M, Nakagawa Y, and Tanimoto K, et al. (2003) Angiotensin II type 2 receptor deficiency exacerbates heart failure and reduces survival after acute myocardial infarction in mice. Circulation 107:2406-2408.

Ahmad S, Cesana F, Lamperti E, Gavras H, and Yu J (2009) Attenuation of angiotensin II-induced hypertension and cardiac hypertrophy in transgenic mice overexpressing a type 1 receptor mutant. Am $J$ Hypertens 22:1320-1325.

Ahmad S, Simmons T, Varagic J, Moniwa N, Chappell MC, and Ferrario CM (2011) Chymase-dependent generation of angiotensin II from angiotensin-(1-12) in human atrial tissue. PLoS One 6:e28501.

Ahmed MS, Øie E, Vinge LE, Yndestad A, Øystein Andersen G, Andersson Y, Attramadal T, and Attramadal H (2004) Connective tissue growth factor-a novel mediator of angiotensin II-stimulated cardiac fibroblast activation in heart failure in rats. J Mol Cell Cardiol 36:393-404.

Ahn S, Shenoy SK, Wei H, and Lefkowitz RJ (2004a) Differential kinetic and spatial patterns of beta-arrestin and G protein-mediated ERK activation by the angiotensin II receptor. J Biol Chem 279:35518-35525.

Ahn S, Wei H, Garrison TR, and Lefkowitz RJ (2004b) Reciprocal regulation of angiotensin receptor-activated extracellular signal-regulated kinases by betaarrestins 1 and $2 . J$ Biol Chem 279:7807-7811.

Ainscough JF, Drinkhill MJ, Sedo A, Turner NA, Brooke DA, Balmforth AJ, and Ball SG (2009) Angiotensin II type-1 receptor activation in the adult heart causes blood pressure-independent hypertrophy and cardiac dysfunction. Cardiovasc Res 81: $592-600$

Akazawa H, Yabumoto C, Yano M, Kudo-Sakamoto Y, and Komuro I (2013) ARB and cardioprotection. Cardiovasc Drugs Ther 27:155-160.

Akishita M, Ito M, Lehtonen JY, Daviet L, Dzau VJ, and Horiuchi M (1999) Expression of the AT2 receptor developmentally programs extracellular signal-regulated kinase activity and influences fetal vascular growth. $J$ Clin Invest 103:63-71.

Albiston AL, Diwakarla S, Fernando RN, Mountford SJ, Yeatman HR, Morgan B, Pham V, Holien JK, Parker MW, and Thompson PE, et al. (2011) Identification and development of specific inhibitors for insulin-regulated aminopeptidase as a new class of cognitive enhancers. Br J Pharmacol 164:37-47.

Albiston AL, Fernando RN, Yeatman HR, Burns P, Ng L, Daswani D, Diwakarla S, Pham V, and Chai SY (2010) Gene knockout of insulin-regulated aminopeptidase: loss of the specific binding site for angiotensin IV and age-related deficit in spatial memory. Neurobiol Learn Mem 93:19-30.

Albiston AL, McDowall SG, Matsacos D, Sim P, Clune E, Mustafa T, Lee J, Mendelsohn FA, Simpson RJ, and Connolly LM, et al. (2001) Evidence that the angiotensin IV (AT(4)) receptor is the enzyme insulin-regulated aminopeptidase. $J$ Biol Chem 276:48623-48626.

Albiston AL, Morton CJ, Ng HL, Pham V, Yeatman HR, Ye S, Fernando RN, De Bundel D, Ascher DB, and Mendelsohn FA, et al. (2008) Identification and characterization of a new cognitive enhancer based on inhibition of insulin-regulated aminopeptidase. FASEB J 22:4209-4217.

Albiston AL, Peck GR, Yeatman HR, Fernando R, Ye S, and Chai SY (2007) Therapeutic targeting of insulin-regulated aminopeptidase: heads and tails? Pharmacol Ther 116:417-427.

Albiston AL, Pederson ES, Burns P, Purcell B, Wright JW, Harding JW, Mendelsohn FA, Weisinger RS, and Chai SY (2004a) Attenuation of scopolamine-induced learning deficits by LVV-hemorphin-7 in rats in the passive avoidance and water maze paradigms. Behav Brain Res 154:239-243.

Albiston AL, Ye S, and Chai SY (2004b) Membrane bound members of the M1 family: more than aminopeptidases. Protein Pept Lett 11:491-500.

Alenina N, Baranova T, Smirnow E, Bader M, Lippoldt A, Patkin E, and Walther T (2002) Cell type-specific expression of the Mas proto-oncogene in testis. J Histo chem Cytochem 50:691-696.

Alenina N, Xu P, Rentzsch B, Patkin EL, and Bader M (2008) Genetically altered animal models for Mas and angiotensin-(1-7). Exp Physiol 93:528-537.

Ali MS, Sayeski PP, Dirksen LB, Hayzer DJ, Marrero MB, and Bernstein KE (1997) Dependence on the motif YIPP for the physical association of Jak2 kinase with the intracellular carboxyl tail of the angiotensin II AT1 receptor. J Biol Chem 272 23382-23388.

Amaya K, Ohta T, Kitagawa H, Kayahara M, Takamura H, Fujimura T, Nishimura G, Shimizu K, and Miwa K (2004) Angiotensin II activates MAP kinase and NF-kappaB through angiotensin II type I receptor in human pancreatic cancer cells. Int $J$ Oncol 25:849-856.

Ambroz C, Clark AJ, and Catt KJ (1991) The mas oncogene enhances angiotensininduced $[\mathrm{Ca} 2+] \mathrm{i}$ responses in cells with pre-existing angiotensin II receptors. Biochim Biophys Acta 1133:107-111.

Anand U, Facer P, Yiangou Y, Sinisi M, Fox M, McCarthy T, Bountra C, Korchev YE, and Anand P (2013) Angiotensin II type 2 receptor (AT2 R) localization and antagonist-mediated inhibition of capsaicin responses and neurite outgrowth in human and rat sensory neurons. Eur J Pain 17:1012-1026.

Andersson H, Demaegdt H, Vauquelin G, Lindeberg G, Karlén A, Hallberg M, Erdélyi M, and Hallberg A. (2010) Disulfide cyclized tripeptide analogues of angiotensin IV as potent and selective inhibitors of insulin-regulated aminopeptidase (IRAP). $J$ Med Chem 53:8059-71.

Andersson H, Demaegdt H, Johnsson A, Vauquelin G, Lindeberg G, Hallberg M, Erdelyi M, Karlen A, and Hallberg A. (2011) Potent macrocyclic inhibitors of insulin-regulated aminopeptidase (IRAP) by olefin ring-closing metathesis. J Med Chem 54:3779-92.
Andreev J, Galisteo ML, Kranenburg O, Logan SK, Chiu ES, Okigaki M, Cary LA, Moolenaar WH, and Schlessinger J (2001) Src and Pyk2 mediate G-protein-coupled receptor activation of epidermal growth factor receptor (EGFR) but are not required for coupling to the mitogen-activated protein (MAP) kinase signaling cascade. J Biol Chem 276:20130-20135.

Andreozzi F, Laratta E, Sciacqua A, Perticone F, and Sesti G (2004) Angiotensin II impairs the insulin signaling pathway promoting production of nitric oxide by inducing phosphorylation of insulin receptor substrate-1 on Ser312 and Ser616 in human umbilical vein endothelial cells. Circ Res 94:1211-1218.

Ansari MJ, Tinckam K, and Chandraker A (2005) Angiotensin II type 1-receptor activating antibodies in renal-allograft rejection. $N$ Engl J Med $\mathbf{3 5 2}$ 2027-2028, author reply $2027-2028$.

Aoki H, Izumo S, and Sadoshima J (1998) Angiotensin II activates RhoA in cardiac myocytes: a critical role of RhoA in angiotensin II-induced premyofibril formation. Circ Res 82:666-676.

Aplin M, Bonde MM, and Hansen JL (2009) Molecular determinants of angiotensin II type 1 receptor functional selectivity. J Mol Cell Cardiol 46:15-24.

Aplin M, Christensen GL, Schneider M, Heydorn A, Gammeltoft S, Kjølbye AL, Sheikh SP, and Hansen JL (2007a) The angiotensin type 1 receptor activates extracellular signal-regulated kinases 1 and 2 by $\mathrm{G}$ protein-dependent and -independent pathways in cardiac myocytes and langendorff-perfused hearts. Basic Clin Pharmacol Toxicol 100:289-295.

Aplin M, Christensen GL, Schneider M, Heydorn A, Gammeltoft S, Kjølbye AL, Sheikh SP, and Hansen JL (2007b) Differential extracellular signal-regulated kinases 1 and 2 activation by the angiotensin type 1 receptor supports distinct phenotypes of cardiac myocytes. Basic Clin Pharmacol Toxicol 100:296-301.

Arsenault J, Cabana J, Fillion D, Leduc R, Guillemette G, Lavigne P, and Escher E (2010a) Temperature dependent photolabeling of the human angiotensin II type 1 receptor reveals insights into its conformational landscape and its activation mechanism. Biochem Pharmacol 80:990-999.

Arsenault J, Lehoux J, Lanthier L, Cabana J, Guillemette G, Lavigne P, Leduc R, and Escher E (2010b) A single-nucleotide polymorphism of alanine to threonine at position 163 of the human angiotensin II type 1 receptor impairs Losartan affinity. Pharmacogenet Genomics 20:377-388.

Audoly LP, Oliverio MI, and Coffman TM (2000) Insights into the functions of type 1 (AT1) angiotensin II receptors provided by gene targeting. Trends Endocrinol Metab 11:263-269.

Axen A, Andersson H, Lindeberg G, Ronnholm H, Kortesmaa J, Demaegdt H, Vauquelin G, Karlen A, and Hallberg M (2007) Small potent ligands to the insulinregulated aminopeptidase (IRAP)/AT(4) receptor. J Pept Sci 13:434-444.

Axen A, Lindeberg G, Demaegdt H, Vauquelin G, Karlen A, and Hallberg M (2006) Cyclic insulin-regulated aminopeptidase (IRAP)/AT4 receptor ligands. J Pept Sci 12:705-713.

Azuma K, Tamura K, Shigenaga A, Wakui H, Masuda S, Tsurumi-Ikeya Y, Tanaka Y, Sakai M, Matsuda M, and Hashimoto T, et al. (2007) Novel regulatory effect of angiotensin II type 1 receptor-interacting molecule on vascular smooth muscle cells. Hypertension 50:926-932.

Bader M, Alenina N, Andrade-Navarro MA, and Santos RA (2014) MAS and its related G protein-coupled receptors, Mrgprs. Pharmacol Rev 66:1080-1105.

Baker KM, Chernin MI, Schreiber T, Sanghi S, Haiderzaidi S, Booz GW, Dostal DE, and Kumar R (2004) Evidence of a novel intracrine mechanism in angiotensin II-induced cardiac hypertrophy. Regul Pept 120:5-13.

Baker KM and Kumar R (2006) Intracellular angiotensin II induces cell proliferation independent of AT1 receptor. Am J Physiol Cell Physiol 291: C995-C1001.

Balmforth AJ, Lee AJ, Bajaj BP, Dickinson CJ, Warburton P, and Ball SG (1995) Functional domains of the C-terminus of the rat angiotensin AT1A receptor. Eur $J$ Pharmacol 291:135-141.

Balmforth AJ, Lee AJ, Warburton P, Donnelly D, and Ball SG (1997) The conformational change responsible for AT1 receptor activation is dependent upon two juxtaposed asparagine residues on transmembrane helices III and VII. J Biol Chem 272:4245-4251.

Barki-Harrington L, Luttrell LM, and Rockman HA (2003) Dual inhibition of betaadrenergic and angiotensin II receptors by a single antagonist: a functional role for receptor-receptor interaction in vivo. Circulation 108:1611-1618.

Bastien NR, Ciuffo GM, Saavedra JM, and Lambert C (1996) Angiotensin II receptor expression in the conduction system and arterial duct of neonatal and adult rat hearts. Regul Pept 63:9-16.

Batenburg WW, Garrelds IM, Bernasconi CC, Juillerat-Jeanneret L, van Kats JP, Saxena PR, and Danser AH (2004) Angiotensin II type 2 receptor-mediated vasodilation in human coronary microarteries. Circulation 109:2296-2301.

Baudin B (2002) Angiotensin II receptor polymorphisms in hypertension. Pharmacogenomic considerations. Pharmacogenomics 3:65-73.

Becker LK, Etelvino GM, Walther T, Santos RA, and Campagnole-Santos MJ (2007) Immunofluorescence localization of the receptor Mas in cardiovascularrelated areas of the rat brain. Am J Physiol Heart Circ Physiol 293:H1416 H1424.

Bedecs K, Elbaz N, Sutren M, Masson M, Susini C, Strosberg AD, and Nahmias C (1997) Angiotensin II type 2 receptors mediate inhibition of mitogen-activated protein kinase cascade and functional activation of SHP-1 tyrosine phosphatase. Biochem J 325:449-454.

Bender E, Buist A, Jurzak M, Langlois X, Baggerman G, Verhasselt P, Ercken M, Guo HQ, Wintmolders C, and Van den Wyngaert I, et al. (2002) Characterization of an orphan $\mathrm{G}$ protein-coupled receptor localized in the dorsal root ganglia reveals adenine as a signaling molecule. Proc Natl Acad Sci USA 99: 8573-8578.

Benetos A, Topouchian J, Ricard S, Gautier S, Bonnardeaux A, Asmar R, Poirier O, Soubrier F, Safar M, and Cambien F (1995) Influence of angiotensin II type 1 receptor polymorphism on aortic stiffness in never-treated hypertensive patients. Hypertension 26:44-47. 
Benndorf R, Böger RH, Ergün S, Steenpass A, and Wieland T (2003) Angiotensin II type 2 receptor inhibits vascular endothelial growth factor-induced migration and in vitro tube formation of human endothelial cells. Circ Res 93:438-447.

Benndorf RA, Krebs C, Hirsch-Hoffmann B, Schwedhelm E, Cieslar G, SchmidtHaupt R, Steinmetz OM, Meyer-Schwesinger C, Thaiss F, and Haddad M, et al. (2009) Angiotensin II type 2 receptor deficiency aggravates renal injury and reduces survival in chronic kidney disease in mice. Kidney Int 75:1039-1049.

Berge KE, Bakken A, Bøhn M, Erikssen J, and Berg K (1997) A DNA polymorphism at the angiotensin II type 1 receptor (AT1R) locus and myocardial infarction. Clin Genet 52:71-76.

Berk BC (2003) Angiotensin type 2 receptor (AT2R): a challenging twin. Sci STKE 2003:PE16.

Berk BC and Corson MA (1997) Angiotensin II signal transduction in vascular smooth muscle: role of tyrosine kinases. Circ Res 80:607-616.

Bernardi S, Zennaro C, Palmisano S, Velkoska E, Sabato N, Toffoli B, Giacomel G, Buri L, Zanconati F, and Bellini G, et al. (2012) Characterization and significance of ACE2 and Mas receptor in human colon adenocarcinoma. J Renin Angiotensin Aldosterone Syst 13:202-209.

Bernier M, Nadiv O, and Kole HK (1995a) Thiol-specific biotinylation of the insulin receptor in permeabilized cells enhances receptor function. Biochemistry 34: 8357-8364.

Bernier SG, Bellemare JM, Escher E, and Guillemette G (1998) Characterization of AT4 receptor from bovine aortic endothelium with photosensitive analogues of angiotensin IV. Biochemistry 37:4280-4287.

Bernier SG, Servant G, Boudreau M, Fournier A, and Guillemette G (1995b) Characterization of a binding site for angiotensin IV on bovine aortic endothelial cells. Eur J Pharmacol 291:191-200.

Bernstein KE, Ali MS, Sayeski PP, Semeniuk D, and Marrero MB (1998) New insights into the cellular signaling of seven transmembrane receptors: the role of tyrosine phosphorylation. Lab Invest 78:3-7.

Beyer AM, Guo DF, and Rahmouni K (2013) Prolonged treatment with angiotensin 1-7 improves endothelial function in diet-induced obesity. $J$ Hypertens 31:730-738.

Bhatnagar A, Unal H, Jagannathan R, Kaveti S, Duan ZH, Yong S, Vasanji A, Kinter M, Desnoyer R, and Karnik SS (2013) Interaction of G-protein $\beta \gamma$ complex with chromatin modulates GPCR-dependent gene regulation. PLoS One 8:e52689.

Bhuiyan MA, Hossain M, Miura S, Nakamura T, Ozaki M, and Nagatomo T (2009) Constitutively active mutant $\mathrm{N} 111 \mathrm{G}$ of angiotensin II type 1 (AT(1)) receptor induces homologous internalization through mediation of AT(1)-receptor antagonist. J Pharmacol Sci 111:227-234.

Bhuiyan MA, Hossain M, Nakamura T, Ozaki M, and Nagatomo T (2010) Internalization of constitutively active N111G MUTANT of AT1 receptor induced by angiotensin II-receptor antagonists candesartan, losartan, and telmisartan: comparison with valsartan. J Pharmacol Sci 112:459-462.

Bienvenu T, Poirier K, Van Esch H, Hamel B, Moraine C, Fryns JP, Ropers HH, Beldjord C, Yntema HG, and Chelly J (2003) Rare polymorphic variants of the AGTR2 gene in boys with non-specific mental retardation. J Med Genet 40:357-359.

Bikkavilli RK, Tsang SY, Tang WM, Sun JX, Ngai SM, Lee SS, Ko WH, Wise H, and Cheung WT (2006) Identification and characterization of surrogate peptide ligand for orphan $\mathrm{G}$ protein-coupled receptor mas using phage-displayed peptide library. Biochem Pharmacol 71:319-337.

Billet S, Aguilar F, Baudry C, and Clauser E (2008) Role of angiotensin II AT1 receptor activation in cardiovascular diseases. Kidney Int 74:1379-1384.

Billet S, Bardin S, Verp S, Baudrie V, Michaud A, Conchon S, Muffat-Joly M, Escoubet B, Souil E, and Hamard G, et al. (2007) Gain-of-function mutant of angiotensin II receptor, type $1 \mathrm{~A}$, causes hypertension and cardiovascular fibrosis in mice. J Clin Invest 117:1914-1925.

Blobel CP (2005) ADAMs: key components in EGFR signalling and development. Nat Rev Mol Cell Biol 6:32-43.

Boerrigter G, Lark MW, Whalen EJ, Soergel DG, Violin JD, and Burnett JC Jr (2011) Cardiorenal actions of TRV120027, a novel ß-arrestin-biased ligand at the angiotensin II type I receptor, in healthy and heart failure canines: a novel therapeutic strategy for acute heart failure. Circ Heart Fail 4:770-778.

Bøgebo R, Horn H, Olsen JV, Gammeltoft S, Jensen LJ, Hansen JL, and Christensen GL (2014) Predicting kinase activity in angiotensin receptor phosphoproteomes based on sequence-motifs and interactions. PLoS One 9:e94672.

Bokemeyer D, Lindemann M, and Kramer HJ (1998) Regulation of mitogen-activated protein kinase phosphatase-1 in vascular smooth muscle cells. Hypertension 32: 661-667.

Bokemeyer D, Schmitz U, and Kramer HJ (2000) Angiotensin II-induced growth of vascular smooth muscle cells requires an Src-dependent activation of the epidermal growth factor receptor. Kidney Int 58:549-558.

Bonnardeaux A, Davies E, Jeunemaitre X, Féry I, Charru A, Clauser E, Tiret L, Cambien F, Corvol P, and Soubrier F (1994) Angiotensin II type 1 receptor gene polymorphisms in human essential hypertension. Hypertension 24:63-69.

Booden MA, Siderovski DP, and Der CJ (2002) Leukemia-associated Rho guanine nucleotide exchange factor promotes $\mathrm{G}$ alpha q-coupled activation of RhoA. Mol Cell Biol 22:4053-4061.

Booz GW and Baker KM (1996) Role of type 1 and type 2 angiotensin receptors in angiotensin II-induced cardiomyocyte hypertrophy. Hypertension 28:635-640.

Borawska M, Kupryszewski G, Witczuk B, and Wiśniewski K (1989) Effects of angiotensin II and its fragments: angiotensin II(3-8)-hexapeptide and angiotensin II (4-8)-pentapeptide on retrieval in passive avoidance situation in rats chronically treated with ethanol. Pol J Pharmacol Pharm 41:227-230.

Bose SK, Gibson W, Giri S, Nath N, and Donald CD (2009) Angiotensin II upregulates PAX2 oncogene expression and activity in prostate cancer via the angiotensin II type I receptor. Prostate 69:1334-1342.

Bosnyak S, Welungoda IK, Hallberg A, Alterman M, Widdop RE, and Jones ES (2010) Stimulation of angiotensin AT2 receptors by the non-peptide agonist, Compound 21 , evokes vasodepressor effects in conscious spontaneously hypertensive rats. Br J Pharmacol 159:709-716.
Botelho-Santos GA, Bader M, Alenina N, and Santos RA (2012) Altered regional blood flow distribution in Mas-deficient mice. Ther Adv Cardiovasc Dis 6:201-211. Bottari SP, King IN, Reichlin S, Dahlstroem I, Lydon N, and de Gasparo M (1992a) The angiotensin AT2 receptor stimulates protein tyrosine phosphatase activity and mediates inhibition of particulate guanylate cyclase. Biochem Biophys Res Commun 183:206-211.

Bottari SP, Obermüller N, Bogdal Y, Zahs KR, and Deschepper CF (1992b) Characterization and distribution of angiotensin II binding sites in fetal and neonatal astrocytes from different rat brain regions. Brain Res 585:372-376.

Bottari SP, Taylor V, King IN, Bogdal Y, Whitebread S, and de Gasparo M (1991) Angiotensin II AT2 receptors do not interact with guanine nucleotide binding proteins. Eur J Pharmacol 207:157-163.

Boucard AA, Roy M, Beaulieu ME, Lavigne P, Escher E, Guillemette G, and Leduc R (2003) Constitutive activation of the angiotensin II type 1 receptor alters the patial proximity of transmembrane 7 to the ligand-binding pocket. $J$ Biol Chem 278:36628-36636.

Boucard AA, Wilkes BC, Laporte SA, Escher E, Guillemette G, and Leduc R (2000) Photolabeling identifies position 172 of the human AT(1) receptor as a ligand contact point: receptor-bound angiotensin II adopts an extended structure. Biochemistry 39:9662-9670.

Bradford CN, Ely DR, and Raizada MK (2010) Targeting the vasoprotective axis of the renin-angiotensin system: a novel strategic approach to pulmonary hypertensive therapy. Curr Hypertens Rep 12:212-219.

Braszko JJ, Kupryszewski G, Witczuk B, and Wiśniewski K (1988) Angiotensin II-(38)-hexapeptide affects motor activity, performance of passive avoidance and a conditioned avoidance response in rats. Neuroscience 27:777-783.

Braun-Dullaeus RC, Mann MJ, Ziegler A, von der Leyen HE, and Dzau VJ (1999) A novel role for the cyclin-dependent kinase inhibitor p27(Kip1) in angiotensin IIstimulated vascular smooth muscle cell hypertrophy. J Clin Invest 104:815-823.

Brechler V, Jones PW, Levens NR, de Gasparo M, and Bottari SP (1993) Agonistic and antagonistic properties of angiotensin analogs at the AT2 receptor in PC12W cells. Regul Pept 44:207-213.

Brede M, Hadamek K, Meinel L, Wiesmann F, Peters J, Engelhardt S, Simm A, Haase A, Lohse MJ, and Hein L (2001) Vascular hypertrophy and increased P70S6 kinase in mice lacking the angiotensin II AT(2) receptor. Circulation 104 2602-2607.

Buisson B, Bottari SP, de Gasparo M, Gallo-Payet N, and Payet MD (1992) The angiotensin AT2 receptor modulates T-type calcium current in non-differentiated NG108-15 cells. FEBS Lett 309:161-164.

Buisson B, Laflamme L, Bottari SP, de Gasparo M, Gallo-Payet N, and Payet MD (1995) A G protein is involved in the angiotensin AT2 receptor inhibition of the T-type calcium current in non-differentiated NG108-15 cells. J Biol Chem 270: $1670-1674$

Bumpus FM, Catt KJ, Chiu AT, DeGasparo M, Goodfriend T, Husain A, Peach MJ, Taylor DG Jr, and Timmermans PB (1991) Nomenclature for angiotensin receptors. A report of the Nomenclature Committee of the Council for High Blood Pressure Research. Hypertension 17:720-721.

Bumpus FM, Schwarz H, and Page IH (1957) Synthesis and pharmacology of the octapeptide angiotonin. Science 125:886-887.

Bunnemann B, Fuxe K, and Ganten D (1992) The brain renin-angiotensin system: localization and general significance. J Cardiovasc Pharmacol 19 (Suppl 6): S51-S62.

Bunnemann B, Fuxe K, Metzger R, Mullins J, Jackson TR, Hanley MR, and Ganten D (1990) Autoradiographic localization of mas proto-oncogene mRNA in adult rat brain using in situ hybridization. Neurosci Lett 114:147-153.

Buraczynska M, Ksiazek P, Zaluska W, Spasiewicz D, Nowicka T, and Ksiazek A (2002) Angiotensin II type 1 receptor gene polymorphism in end-stage renal disease. Nephron 92:51-55.

Busche S, Gallinat S, Bohle RM, Reinecke A, Seebeck J, Franke F, Fink L, Zhu M, Sumners C, and Unger T (2000) Expression of angiotensin AT(1) and $\operatorname{AT}(2)$ receptors in adult rat cardiomyocytes after myocardial infarction. A single-cell reverse transcriptase-polymerase chain reaction study. Am J Pathol 157:605-611.

Caballero R, Gómez R, Moreno I, Nuñez L, González T, Arias C, Guizy M, Valenzuela C, Tamargo J, and Delpón E (2004) Interaction of angiotensin II with the angiotensin type 2 receptor inhibits the cardiac transient outward potassium current. Cardiovasc Res 62:86-95.

Cabello-Verrugio C, Acuña MJ, Morales MG, Becerra A, Simon F, and Brandan E (2011) Fibrotic response induced by angiotensin-II requires NAD $(\mathrm{P}) \mathrm{H}$ oxidaseinduced reactive oxygen species (ROS) in skeletal muscle cells. Biochem Biophys Res Commun 410:665-670.

Campbell SE and Katwa LC (1997) Angiotensin II stimulated expression of transforming growth factor-beta1 in cardiac fibroblasts and myofibroblasts. $J$ Mol Cell Cardiol 29:1947-1958.

Campbell WB, Gebremedhin D, Pratt PF, and Harder DR (1996) Identification of epoxyeicosatrienoic acids as endothelium-derived hyperpolarizing factors. Circ Res 78:415-423.

Canals M, Jenkins L, Kellett E, and Milligan G (2006) Up-regulation of the angiotensin II type 1 receptor by the MAS proto-oncogene is due to constitutive activation of Gq/G11 by MAS. J Biol Chem 281:16757-16767.

Cao Z, Dean R, Wu L, Casley D, and Cooper ME (1999) Role of angiotensin receptor subtypes in mesenteric vascular proliferation and hypertrophy. Hypertension $\mathbf{3 4}$ 408-414.

Carey RM (2012) Functional intracellular renin-angiotensin systems: potential for pathophysiology of disease. Am J Physiol Regul Integr Comp Physiol 302: R479-R481.

Carey RM, Howell NL, Jin XH, and Siragy HM (2001) Angiotensin type 2 receptormediated hypotension in angiotensin type-1 receptor-blocked rats. Hypertension 38:1272-1277.

Carey RM and Siragy HM (2003) The intrarenal renin-angiotensin system and diabetic nephropathy. Trends Endocrinol Metab 14:274-281. 
Caron AZ, Arguin G, and Guillemette G (2003) Angiotensin IV interacts with a juxtamembrane site on AT(4)/IRAP suggesting an allosteric mechanism of enzyme modulation. Regul Pept 113:9-15.

Carstens N, van der Merwe L, Revera M, Heradien M, Goosen A, Brink PA, and Moolman-Smook JC (2011) Genetic variation in angiotensin II type 2 receptor gene influences extent of left ventricular hypertrophy in hypertrophic cardiomyopathy independent of blood pressure. J Renin Angiotensin Aldosterone Syst 12 274-280.

Cary LA, Han DC, Polte TR, Hanks SK, and Guan JL (1998) Identification of p130Cas as a mediator of focal adhesion kinase-promoted cell migration. J Cell Biol 140:211-221.

Castro CH, Santos RA, Ferreira AJ, Bader M, Alenina N, and Almeida AP (2005) Evidence for a functional interaction of the angiotensin-(1-7) receptor Mas with AT1 and AT2 receptors in the mouse heart. Hypertension 46:937-942.

Castro CH, Santos RA, Ferreira AJ, Bader M, Alenina N, and Almeida AP (2006) Effects of genetic deletion of angiotensin-(1-7) receptor Mas on cardiac function during ischemia/reperfusion in the isolated perfused mouse heart. Life Sci 80 264-268.

Chai Y, Jiang X, Ito Y, Bringas P Jr, Han J, Rowitch DH, Soriano P, McMahon AP, and Sucov HM (2000) Fate of the mammalian cranial neural crest during tooth and mandibular morphogenesis. Development 127:1671-1679.

Chaki S, Guo DF, Yamano Y, Ohyama K, Tani M, Mizukoshi M, Shirai H, and Inagami T (1994) Role of carboxyl tail of the rat angiotensin II type 1A receptor in agonist-induced internalization of the receptor. Kidney Int 46:1492-1495.

Chaki S and Inagami T (1992a) Identification and characterization of a new binding site for angiotensin II in mouse neuroblastoma neuro-2A cells. Biochem Biophys Res Commun 182:388-394.

Chaki S and Inagami T (1992b) A newly found angiotensin II receptor subtype mediates cyclic GMP formation in differentiated Neuro-2A cells. Eur J Pharmacol 225:355-356

Chakrabarty A, Blacklock A, Svojanovsky S, and Smith PG (2008) Estrogen elicits dorsal root ganglion axon sprouting via a renin-angiotensin system. Endocrinology 149:3452-3460.

Chamoux E, Breault L, Lehoux JG, and Gallo-Payet N (1999) Involvement of the angiotensin II type 2 receptor in apoptosis during human fetal adrenal gland development. J Clin Endocrinol Metab 84:4722-4730.

Chang RS and Lotti VJ (1990) Two distinct angiotensin II receptor binding sites in rat adrenal revealed by new selective nonpeptide ligands. Mol Pharmacol 37: 347-351.

Chansel D, Vandermeersch S, Pham P, and Ardaillou R (1993) Characterization of [3H]losartan receptors in isolated rat glomeruli. Eur J Pharmacol 247:193-198.

Chaves GZ, Caligiorne SM, Santos RA, Khosla MC, and Campagnole-Santos MJ (2000) Modulation of the baroreflex control of heart rate by angiotensin-(1-7) at the nucleus tractus solitarii of normotensive and spontaneously hypertensive rats. $J$ Hypertens 18:1841-1848.

Che Q and Carmines PK (2002) Angiotensin II triggers EGFR tyrosine kinasedependent $\mathrm{Ca} 2+$ influx in afferent arterioles. Hypertension 40:700-706.

Che ZQ, Gao PJ, Shen WL, Fan CL, Liu JJ, and Zhu DL (2008) Angiotensin IIstimulated collagen synthesis in aortic adventitial fibroblasts is mediated by connective tissue growth factor. Hypertens Res 31:1233-1240.

Chen D, Bassi JK, Walther T, Thomas WG, and Allen AM (2010) Expression of angiotensin type $1 \mathrm{~A}$ receptors in $\mathrm{C} 1$ neurons restores the sympathoexcitation to angiotensin in the rostral ventrolateral medulla of angiotensin type 1A knockout mice. Hypertension 56:143-150.

Chen H and Ikeda SR (2004) Modulation of ion channels and synaptic transmission by a human sensory neuron-specific G-protein-coupled receptor, SNSR4/ mrgX1, heterologously expressed in cultured rat neurons. J Neurosci 24:50445053.

Chen H, Li D, Sawamura T, Inoue K, and Mehta JL (2000a) Upregulation of LOX-1 expression in aorta of hypercholesterolemic rabbits: modulation by losartan. Biochem Biophys Res Commun 276:1100-1104.

Chen HC, Bouchie JL, Perez AS, Clermont AC, Izumo S, Hampe J, and Feener EP (2000b) Role of the angiotensin AT(1) receptor in rat aortic and cardiac PAI-1 gene expression. Arterioscler Thromb Vasc Biol 20:2297-2302.

Chen HJ, Li DY, Saldeen T, Phillips MI, and Mehta JL (2001) Attenuation of tissue P-selectin and MCP-1 expression and intimal proliferation by AT(1) receptor blockade in hyperlipidemic rabbits. Biochem Biophys Res Commun 282 474-479

Chen J, Chen JK, and Harris RC (2012) Angiotensin II induces epithelial-tomesenchymal transition in renal epithelial cells through reactive oxygen species/ Src/caveolin-mediated activation of an epidermal growth factor receptor-extracellular signal-regulated kinase signaling pathway. Mol Cell Biol 32:981-991.

Chen X, Cui Z, Zhang F, Chang W, Chen L, and Liu L (2002) Angiotensin II and cAMP regulate AT(1)-mRNA expression in rat cardiomyocytes by transcriptional mechanism. Eur $J$ Pharmacol 448:1-9.

Chen X, Li W, Yoshida H, Tsuchida S, Nishimura H, Takemoto F, Okubo S, Fogo A, Matsusaka T, and Ichikawa I (1997) Targeting deletion of angiotensin type 1B receptor gene in the mouse. Am $J$ Physiol 272:F299-F304.

Chen XL, Dodd G, Thomas S, Zhang X, Wasserman MA, Rovin BH, and Kunsch C (2006) Activation of Nrf2/ARE pathway protects endothelial cells from oxidant injury and inhibits inflammatory gene expression. Am J Physiol Heart Circ Physiol 290:H1862-H1870.

Chen XL, Tummala PE, Olbrych MT, Alexander RW, and Medford RM (1998) Angiotensin II induces monocyte chemoattractant protein-1 gene expression in rat vascular smooth muscle cells. Circ Res 83:952-959.

Chiu AT, Herblin WF, McCall DE, Ardecky RJ, Carini DJ, Duncia JV, Pease LJ, Wong PC, Wexler RR, and Johnson AL, et al. (1989) Identification of angiotensin II receptor subtypes. Biochem Biophys Res Commun 165:196-203.

Choi SS and Lahn BT (2003) Adaptive evolution of MRG, a neuron-specific gene family implicated in nociception. Genome Res 13:2252-2259.
Chou CH, Chuang LY, Lu CY, and Guh JY (2013) Interaction between TGF- $\beta$ and ACE2-Ang-(1-7)-Mas pathway in high glucose-cultured NRK-52E cells. Mol Cell Endocrinol 366:21-30.

Christensen GL, Kelstrup CD, Lyngsø C, Sarwar U, Bøgebo R, Sheikh SP, Gammeltoft S, Olsen JV, and Hansen JL (2010) Quantitative phosphoproteomics dissection of seven-transmembrane receptor signaling using full and biased agonists. Mol Cell Proteomics 9:1540-1553.

Chua TC, Sarkar A, Saxena A, Glenn D, Zhao J, and Morris DL (2010) Long-term outcome of image-guided percutaneous radiofrequency ablation of lung metastases: an open-labeled prospective trial of 148 patients. Ann Oncol 21:2017-2022.

Clément M, Cabana J, Holleran BJ, Leduc R, Guillemette G, Lavigne P, and Escher E (2009) Activation induces structural changes in the liganded angiotensin II type 1 receptor. J Biol Chem 284:26603-26612.

Clément M, Chamberland C, Pérodin J, Leduc R, Guillemette G, and Escher E (2006) The active and the inactive form of the hAT1 receptor have an identical ligandbinding environment: an MPA study on a constitutively active angiotensin II receptor mutant. J Recept Signal Transduct Res 26:417-433.

Clément M, Martin SS, Beaulieu ME, Chamberland C, Lavigne P, Leduc R, Guillemette G, and Escher E (2005) Determining the environment of the ligand binding pocket of the human angiotensin II type I (hAT1) receptor using the methionine proximity assay. J Biol Chem 280:27121-27129.

Coleman JK, Krebs LT, Hamilton TA, Ong B, Lawrence KA, Sardinia MF, Harding JW, and Wright JW (1998) Autoradiographic identification of kidney angiotensin IV binding sites and angiotensin IV-induced renal cortical blood flow changes in rats. Peptides 19:269-277.

Coll E, Campos B, González-Núñez D, Botey A, and Poch E (2003) Association between the A1166C polymorphism of the angiotensin II receptor type 1 and progression of chronic renal insufficiency. $J$ Nephrol 16:357-364.

Colmenero J, Bataller R, Sancho-Bru P, Domínguez M, Moreno M, Forns X, Bruguera $\mathrm{M}$, Arroyo V, Brenner DA, and Ginès $\mathrm{P}$ (2009) Effects of losartan on hepatic expression of nonphagocytic NADPH oxidase and fibrogenic genes in patients with chronic hepatitis C. Am J Physiol Gastrointest Liver Physiol 297:G726-G734.

Conchon S, Peltier N, Corvol P, and Clauser E (1998) A noninternalized nondesensitized truncated AT1A receptor transduces an amplified ANG II signal. Am $J$ Physiol 274:E336-E345.

Cook JL and Re RN (2012) Lessons from in vitro studies and a related intracellular angiotensin II transgenic mouse model. Am J Physiol Regul Integr Comp Physiol 302:R482-R493.

Cook JL, Re RN, deHaro DL, Abadie JM, Peters M, and Alam J (2008) The trafficking protein GABARAP binds to and enhances plasma membrane expression and function of the angiotensin II type 1 receptor. Circ Res 102:1539-1547.

Coppey LJ, Davidson EP, Rinehart TW, Gellett JS, Oltman CL, Lund DD, and Yorek MA (2006) ACE inhibitor or angiotensin II receptor antagonist attenuates diabetic neuropathy in streptozotocin-induced diabetic rats. Diabetes 55:341-348.

Correa SA, Zalcberg H, Han SW, Oliveira L, Costa-Neto CM, Paiva AC, and Shimuta SI (2002) Aliphatic amino acids in helix VI of the AT(1) receptor play a relevant role in agonist binding and activity. Regul Pept 106:33-38.

Costa MA, Lopez Verrilli MA, Gomez KA, Nakagawa P, Peña C, Arranz C, and Gironacci MM (2010) Angiotensin-(1-7) upregulates cardiac nitric oxide synthase in spontaneously hypertensive rats. Am J Physiol Heart Circ Physiol 299 H1205-H1211.

Côté F, Do TH, Laflamme L, Gallo JM, and Gallo-Payet N (1999) Activation of the AT (2) receptor of angiotensin II induces neurite outgrowth and cell migration in microexplant cultures of the cerebellum. J Biol Chem 274:31686-31692.

Cowling RT, Gurantz D, Peng J, Dillmann WH, and Greenberg BH (2002) Transcription factor NF-kappa B is necessary for up-regulation of type 1 angiotensin II receptor mRNA in rat cardiac fibroblasts treated with tumor necrosis factor-alpha or interleukin-1 beta. $J$ Biol Chem 277:5719-5724.

Cox BE, Ipson MA, Shaul PW, Kamm KE, and Rosenfeld CR (1993) Myometrial angiotensin II receptor subtypes change during ovine pregnancy. J Clin Invest $\mathbf{9 2}$ $2240-2248$

Crawford DC, Chobanian AV, and Brecher P (1994) Angiotensin II induces fibronectin expression associated with cardiac fibrosis in the rat. Circ Res 74:727739

Criscione L, Thomann H, Whitebread S, de Gasparo M, Bühlmayer P, Herold P, Ostermayer F, and Kamber B (1990) Binding characteristics and vascular effects of various angiotensin II antagonists. J Cardiovasc Pharmacol 16 (Suppl 4):S56-S59

Cruse CW, Wells KE, and Reintgen DS (1992) Treatment of the primary in malignant melanoma of the skin. Ann Plast Surg 28:22-25.

Cui T, Nakagami H, Iwai M, Takeda Y, Shiuchi T, Tamura K, Daviet L, and Horiuch M (2000) ATRAP, novel AT1 receptor associated protein, enhances internalization of AT1 receptor and inhibits vascular smooth muscle cell growth. Biochem Biophys Res Commun 279:938-941.

D'Amore A, Black MJ, and Thomas WG (2005) The angiotensin II type 2 receptor causes constitutive growth of cardiomyocytes and does not antagonize angiotensin II type 1 receptor-mediated hypertrophy. Hypertension 46:1347-1354.

da Costa Gonçalves AC, Leite R, Fraga-Silva RA, Pinheiro SV, Reis AB, Reis FM, Touyz RM, Webb RC, Alenina N, and Bader M, et al. (2007) Evidence that the vasodilator angiotensin-(1-7)-Mas axis plays an important role in erectile function. Am J Physiol Heart Circ Physiol 293:H2588-H2596.

da Silva AQ, Fontes MA, and Kanagy NL (2011) Chronic infusion of angiotensin receptor antagonists in the hypothalamic paraventricular nucleus prevents hypertension in a rat model of sleep apnea. Brain Res 1368:231-238.

da Silveira KD, Pompermayer Bosco KS, Diniz LR, Carmona AK, Cassali GD, BrunaRomero O, de Sousa LP, Teixeira MM, Santos RA, and Simões e Silva AC, et al. (2010) ACE2-angiotensin-(1-7)-Mas axis in renal ischaemia/reperfusion injury in rats. Clin Sci (Lond) 119:385-394.

Dale LB, Seachrist JL, Babwah AV, and Ferguson SS (2004) Regulation of angiotensin II type $1 \mathrm{~A}$ receptor intracellular retention, degradation, and recycling by Rab5, Rab7, and Rab11 GTPases. J Biol Chem 279:13110-13118. 
Daniels D, Yee DK, Faulconbridge LF, and Fluharty SJ (2005) Divergent behavioral roles of angiotensin receptor intracellular signaling cascades. Endocrinology 146 $5552-5560$.

Darimont C, Vassaux G, Ailhaud G, and Negrel R (1994) Differentiation of preadipose cells: paracrine role of prostacyclin upon stimulation of adipose cells by angiotensin-II. Endocrinology 135:2030-2036.

Dasgupta C and Zhang L (2011) Angiotensin II receptors and drug discovery in cardiovascular disease. Drug Discov Today 16:22-34.

Daugherty A, Manning MW, and Cassis LA (2000) Angiotensin II promotes atherosclerotic lesions and aneurysms in apolipoprotein E-deficient mice. J Clin Invest 105:1605-1612.

Daugherty A, Rateri DL, Lu H, Inagami T, and Cassis LA (2004) Hypercholesterolemia stimulates angiotensin peptide synthesis and contributes to atherosclerosis through the AT1A receptor. Circulation 110:3849-3857.

Davenport AP, Alexander SP, Sharman JL, Pawson AJ, Benson HE, Monaghan AE, Liew WC, Mpamhanga CP, Bonner TI, and Neubig RR, et al. (2013) International Union of Basic and Clinical Pharmacology. LXXXVIII. G protein-coupled receptor list: recommendations for new pairings with cognate ligands. Pharmacol Rev 65 967-986

David A (1976) [In memory of Frantisek Suntych, M.D., C.Sc]. Cas Lek Cesk 115:223

Daviet L, Lehtonen JY, Tamura K, Griese DP, Horiuchi M, and Dzau VJ (1999) Cloning and characterization of ATRAP, a novel protein that interacts with the angiotensin II type 1 receptor. J Biol Chem 274:17058-17062.

Dean NM and Boynton AL (1990) Angiotensin II causes phosphatidylinositol turnover and increases 1,2-diacylglycerol mass but is not mitogenic in rat liver T51B cells. Biochem J 269:347-352.

de Boer RA, Pinto YM, Suurmeijer AJ, Pokharel S, Scholtens E, Humler M, Saavedra JM, Boomsma F, van Gilst WH, and van Veldhuisen DJ (2003) Increased expression of cardiac angiotensin II type 1 (AT(1)) receptors decreases myocardial microvessel density after experimental myocardial infarction. Cardiovasc Res 57:434-442.

De Bundel D, Smolders I, Vanderheyden P, and Michotte Y (2008) Ang II and Ang IV: unraveling the mechanism of action on synaptic plasticity, memory, and epilepsy. CNS Neurosci Ther 14:315-339.

De Bundel D, Smolders I, Yang R, Albiston AL, Michotte Y, and Chai SY (2009) Angiotensin IV and LVV-haemorphin 7 enhance spatial working memory in rats: effects on hippocampal glucose levels and blood flow. Neurobiol Learn Mem 92:19-26.

Dechend R, Homuth V, Wallukat G, Kreuzer J, Park JK, Theuer J, Juepner A, Gulba DC, Mackman N, and Haller H, et al. (2000) AT(1) receptor agonistic antibodies from preeclamptic patients cause vascular cells to express tissue factor. Circulation 101:2382-2387.

Dechend R, Müller DN, Wallukat G, Homuth V, Krause M, Dudenhausen J, and Luft FC (2004) AT1 receptor agonistic antibodies, hypertension, and preeclampsia. Semin Nephrol 24:571-579.

Dechend R, Viedt C, Müller DN, Ugele B, Brandes RP, Wallukat G, Park JK, Janke J, Barta P and Theuer J, et al. (2003) AT1 receptor agonistic antibodies from preeclamptic patients stimulate NADPH oxidase. Circulation 107:1632-1639.

De Gasparo M (2002) [AT(1) and AT(2) angiotensin II receptors: key features]. Drugs 62:1-10.

de Gasparo M, Catt KJ, Inagami T, Wright JW, and Unger T (2000) International Union of Pharmacology. XXIII. The angiotensin II receptors. Pharmacol Rev 52: $415-472$.

de Kloet AD, Wang L, Ludin JA, Smith JA, Pioquinto DJ, Hiller H, Steckelings UM, Scheuer DA, Sumners C, and Krause EG (2014) Reporter mouse strain provides a novel look at angiotensin type-2 receptor distribution in the central nervous system. Brain Struct Funct, [published ahead of print].

Demaegdt H, Gard P, De Backer JP, Lukaszuk A, Szemenyei E, Tóth G, Tourwé D, and Vauquelin G (2011) Binding of "AT4 receptor" ligands to insulin regulated aminopeptidase (IRAP) in intact Chinese hamster ovary cells. Mol Cell Endocrinol 339:34-44.

Demaegdt H, Laeremans H, De Backer JP, Mosselmans S, Le MT, Kersemans V, Michotte Y, Vauquelin G, and Vanderheyden PM (2004a) Synergistic modulation of cystinyl aminopeptidase by divalent cation chelators. Biochem Pharmacol 68 893-900.

Demaegdt H, Lenaerts PJ, Swales J, De Backer JP, Laeremans H, Le MT, Kersemans K, Vogel LK, Michotte Y, and Vanderheyden P, et al. (2006) Angiotensin AT4 receptor ligand interaction with cystinyl aminopeptidase and aminopeptidase N: [125I]Angiotensin IV only binds to the cystinyl aminopeptidase apo-enzyme. Eur J Pharmacol 546:19-27.

Demaegdt H, Vanderheyden P, De Backer JP, Mosselmans S, Laeremans H, Le MT, Kersemans V, Michotte Y, and Vauquelin G (2004b) Endogenous cystinyl aminopeptidase in Chinese hamster ovary cells: characterization by [125I]Ang IV binding and catalytic activity. Biochem Pharmacol 68:885-892.

De Paolis P, Porcellini A, Savoia C, Lombardi A, Gigante B, Frati G, Rubattu S, Musumeci B, and Volpe M (2002) Functional cross-talk between angiotensin II and epidermal growth factor receptors in NIH3T3 fibroblasts. $J$ Hypertens 20:693-699.

De Souza AM, Lopes AG, Pizzino CP, Fossari RN, Miguel NC, Cardozo FP, Abi-Abib R, Fernandes MS, Santos DP, and Caruso-Neves C (2004) Angiotensin II and angiotensin-(1-7) inhibit the inner cortex Na+-ATPase activity through AT2 receptor. Regul Pept 120:167-175.

Deshayes F and Nahmias C (2005) Angiotensin receptors: a new role in cancer? Trends Endocrinol Metab 16:293-299.

Devynck MA, Pernollet MG, Macdonald GJ, Matthews PG, Raisman RS, and Meyer P (1978) Alterations of adrenal and uterine angiotensin II receptors during variation of sodium intake and/or experimental hypertension. Clin Sci Mol Med Suppl 4: 171S-174S

DeWire SM, Ahn S, Lefkowitz RJ, and Shenoy SK (2007) Beta-arrestins and cell signaling. Annu Rev Physiol 69:483-510.

DeWire SM, Kim J, Whalen EJ, Ahn S, Chen M, and Lefkowitz RJ (2008) Betaarrestin-mediated signaling regulates protein synthesis. J Biol Chem 283: 10611-10620.
DeWire SM and Violin JD (2011) Biased ligands for better cardiovascular drugs: dissecting G-protein-coupled receptor pharmacology. Circ Res 109:205-216.

Dias-Peixoto MF, Santos RA, Gomes ER, Alves MN, Almeida PW, Greco L, Rosa M, Fauler B, Bader M, and Alenina N, et al. (2008) Molecular mechanisms involved in the angiotensin-(1-7)/Mas signaling pathway in cardiomyocytes. Hypertension 52: $542-548$.

Díaz-Rodríguez E, Montero JC, Esparís-Ogando A, Yuste L, and Pandiella A (2002) Extracellular signal-regulated kinase phosphorylates tumor necrosis factor alphaconverting enzyme at threonine 735: a potential role in regulated shedding. $M o$ Biol Cell 13:2031-2044.

Diep QN, El Mabrouk M, Touyz RM, and Schiffrin EL (2001) Expression of cell cycle proteins in blood vessels of angiotensin II-infused rats: role of AT(1) receptors. Hypertension 37:604-608.

Dikic I, Tokiwa G, Lev S, Courtneidge SA, and Schlessinger J (1996) A role for Pyk2 and Src in linking G-protein-coupled receptors with MAP kinase activation. Nature 383:547-550.

Dinh DT, Frauman AG, Johnston CI, and Fabiani ME (2001) Angiotensin receptors: distribution, signalling and function. Clin Sci (Lond) 100:481-492.

Doan TN, Ali MS, and Bernstein KE (2001) Tyrosine kinase activation by the angiotensin II receptor in the absence of calcium signaling. $J$ Biol Chem 276: 20954-20958.

Domazet I, Holleran BJ, Martin SS, Lavigne P, Leduc R, Escher E, and Guillemette $\mathrm{G}$ (2009a) The second transmembrane domain of the human type 1 angiotensin II receptor participates in the formation of the ligand binding pocket and undergoes integral pivoting movement during the process of receptor activation. J Biol Chem 284:11922-11929.

Domazet I, Martin SS, Holleran BJ, Morin ME, Lacasse P, Lavigne P, Escher E, Leduc R, and Guillemette G (2009b) The fifth transmembrane domain of angiotensin II Type 1 receptor participates in the formation of the ligand-binding pocket and undergoes a counterclockwise rotation upon receptor activation. J Biol Chem 284:31953-31961.

Dong X, Han S, Zylka MJ, Simon MI, and Anderson DJ (2001) A diverse family of GPCRs expressed in specific subsets of nociceptive sensory neurons. Cell 106:619-632. Dörffel Y, Wallukat G, Bochnig N, Homuth V, Herberg M, Dörffel W, Pruss A, Chaoui R, and Scholze J (2003) Agonistic AT(1) receptor autoantibodies and monocyte stimulation in hypertensive patients. Am J Hypertens 16:827-833.

Dorn GW 2nd and Force T (2005) Protein kinase cascades in the regulation of cardiac hypertrophy. J Clin Invest 115:527-537.

Dragun D, Müller DN, Bräsen JH, Fritsche L, Nieminen-Kelhä M, Dechend R, Kintscher U, Rudolph B, Hoebeke J, and Eckert D, et al. (2005) Angiotensin II type 1-receptor activating antibodies in renal-allograft rejection. $N$ Engl $J$ Med 352: $558-569$.

Dudley DT, Hubbell SE, and Summerfelt RM (1991) Characterization of angiotensin II (AT2) binding sites in R3T3 cells. Mol Pharmacol 40:360-367.

Dudley DT, Panek RL, Major TC, Lu GH, Bruns RF, Klinkefus BA, Hodges JC, and Weishaar RE (1990) Subclasses of angiotensin II binding sites and their functional significance. Mol Pharmacol 38:370-377.

Dudley DT and Summerfelt RM (1993) Regulated expression of angiotensin II (AT2) binding sites in R3T3 cells. Regul Pept 44:199-206.

Dugourd C, Gervais M, Corvol P, and Monnot C (2003) Akt is a major downstream target of PI3-kinase involved in angiotensin II-induced proliferation. Hypertension 41:882-890.

Duncan JA, Scholey JW, and Miller JA (2001) Angiotensin II type 1 receptor gene polymorphisms in humans: physiology and pathophysiology of the genotypes. Curr Opin Nephrol Hypertens 10:111-116.

Durand MJ, Raffai G, Weinberg BD, and Lombard JH (2010) Angiotensin-(1-7) and low-dose angiotensin II infusion reverse salt-induced endothelial dysfunction via different mechanisms in rat middle cerebral arteries. Am J Physiol Heart Circ Physiol 299:H1024-H1033.

Durik M, van Veghel R, Kuipers A, Rink R, Haas Jimoh Akanbi M, Moll G, Danser $\mathrm{AH}$, and Roks AJ (2012) The effect of the thioether-bridged, stabilized Angiotensin(1-7) analogue cyclic ang-(1-7) on cardiac remodeling and endothelial function in rats with myocardial infarction. Int $J$ Hypertens 2012:536426.

Duron E and Hanon O (2010) Antihypertensive treatments, cognitive decline, and dementia. J Alzheimers Dis 20:903-914.

Dzau VJ and Gibbons GH (1987) Autocrine-paracrine mechanisms of vascular myocytes in systemic hypertension. Am J Cardiol 60:99I-103I.

Ebert BL, Firth JD, and Ratcliffe PJ (1995) Hypoxia and mitochondrial inhibitors regulate expression of glucose transporter-1 via distinct Cis-acting sequences. $J$ Biol Chem 270:29083-29089.

Egami K, Murohara T, Shimada T, Sasaki K, Shintani S, Sugaya T, Ishii M, Akagi T, Ikeda H, and Matsuishi T, et al. (2003) Role of host angiotensin II type 1 receptor in tumor angiogenesis and growth. J Clin Invest 112:67-75.

Eguchi S, Dempsey PJ, Frank GD, Motley ED, and Inagami T (2001) Activation of MAPKs by angiotensin II in vascular smooth muscle cells. Metalloproteasedependent EGF receptor activation is required for activation of ERK and p38 MAPK but not for JNK. J Biol Chem 276:7957-7962

Eguchi S, Iwasaki H, Inagami T, Numaguchi K, Yamakawa T, Motley ED, Owada KM, Marumo F, and Hirata Y (1999) Involvement of PYK2 in angiotensin II signaling of vascular smooth muscle cells. Hypertension 33:201-206.

Eguchi S, Matsumoto T, Motley ED, Utsunomiya H, and Inagami T (1996) Identification of an essential signaling cascade for mitogen-activated protein kinase activation by angiotensin II in cultured rat vascular smooth muscle cells. Possible requirement of Gq-mediated p21ras activation coupled to a $\mathrm{Ca} 2+/$ calmodulinsensitive tyrosine kinase. J Biol Chem 271:14169-14175.

Eguchi S, Numaguchi K, Iwasaki H, Matsumoto T, Yamakawa T, Utsunomiya H, Motley ED, Kawakatsu H, Owada KM, and Hirata Y, et al. (1998) Calciumdependent epidermal growth factor receptor transactivation mediates the angiotensin II-induced mitogen-activated protein kinase activation in vascular smooth muscle cells. J Biol Chem 273:8890-8896. 
Elbaz N, Bedecs K, Masson M, Sutren M, Strosberg AD, and Nahmias C (2000) Functional trans-inactivation of insulin receptor kinase by growth-inhibitory angiotensin II AT2 receptor. Mol Endocrinol 14:795-804.

Elliott DF and Peart WS (1957) The amino acid sequence in a hypertensin. Biochem $J$ 65:246-254.

Ellis B, Li XC, Miguel-Qin E, Gu V, and Zhuo JL (2012) Evidence for a functional intracellular angiotensin system in the proximal tubule of the kidney. Am J Physio Regul Integr Comp Physiol 302:R494-R509.

Elton TS and Martin MM (2003) Alternative splicing: a novel mechanism to fine-tune the expression and function of the human AT1 receptor. Trends Endocrinol Metab 14:66-71.

Elton TS and Martin MM (2007) Angiotensin II type 1 receptor gene regulation: transcriptional and posttranscriptional mechanisms. Hypertension 49:953-961.

Enjoji M, Kotoh K, Kato M, Higuchi N, Kohjima M, Nakashima M, and Nakamuta M (2008) Therapeutic effect of ARBs on insulin resistance and liver injury in patients with NAFLD and chronic hepatitis C: a pilot study. Int J Mol Med 22:521-527.

Erdmann J, Riedel K, Rohde K, Folgmann I, Wienker T, Fleck E, and RegitzZagrosek V (1999) Characterization of polymorphisms in the promoter of the human angiotensin II subtype 1 (AT1) receptor gene. Ann Hum Genet 63 369-374.

Escobar E, Rodríguez-Reyna TS, Arrieta O, and Sotelo J (2004) Angiotensin II, cell proliferation and angiogenesis regulator: biologic and therapeutic implications in cancer. Curr Vasc Pharmacol 2:385-399.

Esteban V, Heringer-Walther S, Sterner-Kock A, de Bruin R, van den Engel S, Wang Y, Mezzano S, Egido J, Schultheiss HP, and Ruiz-Ortega M, et al. (2009) Angiotensin-(1-7) and the g protein-coupled receptor MAS are key players in renal inflammation. PLoS One 4:e5406.

Esteban V, Ruperez M, Sánchez-López E, Rodríguez-Vita J, Lorenzo O, Demaegdt H, Vanderheyden P, Egido J, and Ruiz-Ortega M (2005) Angiotensin IV activates the nuclear transcription factor-kappaB and related proinflammatory genes in vascular smooth muscle cells. Circ Res 96:965-973.

Evanko SP, Raines EW, Ross R, Gold LI, and Wight TN (1998) Proteoglycan distribution in lesions of atherosclerosis depends on lesion severity, structural characteristics, and the proximity of platelet-derived growth factor and transforming growth factor-beta. Am J Pathol 152:533-546.

Everett AD, Stoops TD, Nairn AC, and Brautigan D (2001) Angiotensin II regulates phosphorylation of translation elongation factor-2 in cardiac myocytes. $A m J$ Physiol Heart Circ Physiol 281:H161-H167.

Falcón BL, Veerasingham SJ, Sumners C, and Raizada MK (2005) Angiotensin II type 2 receptor-mediated gene expression profiling in human coronary artery endothelial cells. Hypertension 45:692-697.

Faria-Silva R, Duarte FV, and Santos RA (2005) Short-term angiotensin(1-7) receptor MAS stimulation improves endothelial function in normotensive rats. Hypertension 46:948-952.

Faure C, Mnie-Filali O, and Haddjeri N (2006a) Long-term adaptive changes induced by serotonergic antidepressant drugs. Expert Rev Neurother 6:235-245.

Faure C, Mnie-Filali O, Scarna H, Debonnel G, and Haddjeri N (2006b) Effects of the 5-HT7 receptor antagonist SB-269970 on rat hormonal and temperature responses to the 5-HT1A/7 receptor agonist 8-OH-DPAT. Neurosci Lett 404: $122-126$.

Faure S, Bureau A, Oudart N, Javellaud J, Fournier A, and Achard JM (2008) Protective effect of candesartan in experimental ischemic stroke in the rat mediated by AT2 and AT4 receptors. $J$ Hypertens 26:2008-2015.

Feener EP, Northrup JM, Aiello LP, and King GL (1995) Angiotensin II induces plasminogen activator inhibitor-1 and -2 expression in vascular endothelial and smooth muscle cells. J Clin Invest 95:1353-1362.

Feng YH, Miura S, Husain A, and Karnik SS (1998) Mechanism of constitutive activation of the AT1 receptor: influence of the size of the agonist switch binding residue Asn(111). Biochemistry 37:15791-15798.

Feng YH, Noda K, Saad Y, Liu XP, Husain A, and Karnik SS (1995) The docking of Arg2 of angiotensin II with Asp281 of AT1 receptor is essential for full agonism. $J$ Biol Chem 270:12846-12850.

Feng YH, Saad Y, and Karnik SS (2000) Reversible inactivation of AT(2) angiotensin II receptor from cysteine-disulfide bond exchange. FEBS Lett 484:133-138.

Feng YH, Sun Y, and Douglas JG (2002) Gbeta gamma -independent constitutive association of Galpha s with SHP-1 and angiotensin II receptor AT2 is essential in AT2-mediated ITIM-independent activation of SHP-1. Proc Natl Acad Sci USA 99: 12049-12054.

Feng YH, Zhou L, Qiu R, and Zeng R (2005) Single mutations at Asn295 and Leu305 in the cytoplasmic half of transmembrane alpha-helix domain 7 of the AT1 receptor induce promiscuous agonist specificity for angiotensin II fragments: a pseudoconstitutive activity. Mol Pharmacol 68:347-355.

Fernando RN, Albiston AL, and Chai SY (2008) The insulin-regulated aminopeptidase IRAP is colocalised with GLUT4 in the mouse hippocampus-potential role in modulation of glucose uptake in neurones? Eur J Neurosci 28:588-598.

Fernando RN, Larm J, Albiston AL, and Chai SY (2005) Distribution and cellular localization of insulin-regulated aminopeptidase in the rat central nervous system. J Comp Neurol 487:372-390.

Fernando RN, Luff SE, Albiston AL, and Chai SY (2007) Sub-cellular localization of insulin-regulated membrane aminopeptidase, IRAP to vesicles in neurons. $J$ Neurochem 102:967-976.

Ferrario CM, Brosnihan KB, Diz DI, Jaiswal N, Khosla MC, Milsted A, and Tallant EA (1991) Angiotensin-(1-7): a new hormone of the angiotensin system. Hypertension 18(5, Suppl)III126-III133.

Ferreira AJ, Moraes PL, Foureaux G, Andrade AB, Santos RA, and Almeida AP (2011) The angiotensin-(1-7)/Mas receptor axis is expressed in sinoatrial node cells of rats. J Histochem Cytochem 59:761-768.

Fessart D, Simaan M, and Laporte SA (2005) c-Src regulates clathrin adapter protein 2 interaction with beta-arrestin and the angiotensin II type 1 receptor during clathrin- mediated internalization. Mol Endocrinol 19:491-503.
Figueroa JE and Vijayagopal P (2002) Angiotensin II stimulates synthesis of vascular smooth muscle cell proteoglycans with enhanced low density lipoprotein binding properties. Atherosclerosis 162:261-268.

Fillion D, Cabana J, Guillemette G, Leduc R, Lavigne P, and Escher E (2013) Structure of the human angiotensin II type 1 (AT1) receptor bound to angiotensin II from multiple chemoselective photoprobe contacts reveals a unique peptide binding mode. J Biol Chem 288:8187-8197.

Fischer OM, Hart S, Gschwind A, Prenzel N, and Ullrich A (2004) Oxidative and osmotic stress signaling in tumor cells is mediated by ADAM proteases and heparin-binding epidermal growth factor. Mol Cell Biol 24:5172-5183.

Fischer TA, Singh K, O'Hara DS, Kaye DM, and Kelly RA (1998) Role of AT1 and AT2 receptors in regulation of MAPKs and MKP-1 by ANG II in adult cardiac myocytes. Am J Physiol 275:H906-H916.

Fitzgerald SM, Evans RG, Bergström G, and Anderson WP (1999) Renal hemodynamic responses to intrarenal infusion of ligands for the putative angiotensin IV receptor in anesthetized rats. J Cardiovasc Pharmacol 34:206-211.

Flores-Muñoz M, Godinho BM, Almalik A, and Nicklin SA (2012) Adenoviral delivery of angiotensin-(1-7) or angiotensin-(1-9) inhibits cardiomyocyte hypertrophy via the mas or angiotensin type 2 receptor. PLoS One 7:e45564.

Folli F, Kahn CR, Hansen H, Bouchie JL, and Feener EP (1997) Angiotensin II inhibits insulin signaling in aortic smooth muscle cells at multiple levels. A potential role for serine phosphorylation in insulin/angiotensin II crosstalk. $J$ Clin Invest 100:2158-2169.

Fontes MA, Silva LC, Campagnole-Santos MJ, Khosla MC, Guertzenstein PG, and Santos RA (1994) Evidence that angiotensin-(1-7) plays a role in the central control of blood pressure at the ventro-lateral medulla acting through specific receptors. Brain Res 665:175-180.

Fraga-Silva RA, Pinheiro SV, Gonçalves AC, Alenina N, Bader M, and Santos RA (2008) The antithrombotic effect of angiotensin-(1-7) involves mas-mediated NO release from platelets. $\mathrm{Mol} \mathrm{Med}$ 14:28-35

Frank GD, Saito S, Motley ED, Sasaki T, Ohba M, Kuroki T, Inagami T, and Eguchi S (2002) Requirement of $\mathrm{Ca}(2+)$ and PKCdelta for Janus kinase 2 activation by angiotensin II: involvement of PYK2. Mol Endocrinol 16:367-377.

Fredj S, Bescond J, Louault C, Delwail A, Lecron JC, and Potreau D (2005) Role of interleukin-6 in cardiomyocyte/cardiac fibroblast interactions during myocyte hypertrophy and fibroblast proliferation. J Cell Physiol 204:428-436.

Fu ML, Herlitz H, Schulze W, Wallukat G, Micke P, Eftekhari P, Sjögren KG, Hjalmarson A, Müller-Esterl W, and Hoebeke J (2000) Autoantibodies against the angiotensin receptor (AT1) in patients with hypertension. $J$ Hypertens 18:945-953.

Fujino M, Miura S, Kiya Y, Tominaga Y, Matsuo Y, Karnik SS, and Saku K (2010) A small difference in the molecular structure of angiotensin II receptor blockers induces AT(1) receptor-dependent and -independent beneficial effects. Hypertens Res 33:1044-1052.

Fujita M, Hayashi I, Yamashina S, Fukamizu A, Itoman M, and Majima M (2005) Angiotensin type 1a receptor signaling-dependent induction of vascular endothelial growth factor in stroma is relevant to tumor-associated angiogenesis and tumor growth. Carcinogenesis 26:271-279.

Gáborik Z, Jagadeesh G, Zhang M, Spät A, Catt KJ, and Hunyady L (2003) The role of a conserved region of the second intracellular loop in AT1 angiotensin receptor activation and signaling. Endocrinology 144:2220-2228.

Gabrielsen A, Lawler PR, Yongzhong W, Steinbrüchel D, Blagoja D, Paulsson-Berne G, Kastrup J, and Hansson GK (2007) Gene expression signals involved in ischemic injury, extracellular matrix composition and fibrosis defined by global mRNA profiling of the human left ventricular myocardium. $J$ Mol Cell Cardiol 42 870-883.

Galandrin S, Oligny-Longpré G, and Bouvier M (2007) The evasive nature of drug efficacy: implications for drug discovery. Trends Pharmacol Sci 28:423-430.

Gallagher PE, Ferrario CM, and Tallant EA (2008) Regulation of ACE2 in cardiac myocytes and fibroblasts. Am J Physiol Heart Circ Physiol 295:H2373-H2379.

Gallinat S, Busche S, Schütze S, Krönke M, and Unger T (1999) AT2 receptor stimulation induces generation of ceramides in PC12W cells. FEBS Lett 443:75-79.

Gallinat S, Yu M, Dorst A, Unger T, and Herdegen T (1998) Sciatic nerve transection evokes lasting up-regulation of angiotensin AT2 and AT1 receptor mRNA in adult rat dorsal root ganglia and sciatic nerves. Brain Res Mol Brain Res 57:111-122.

Gao BB, Hansen H, Chen HC, and Feener EP (2006) Angiotensin II stimulates phosphorylation of an ectodomain-truncated platelet-derived growth factor receptor-beta and its binding to class IA PI3K in vascular smooth muscle cells. Biochem J 397:337-344.

García-Caballero A, Olivares-Reyes JA, Catt KJ, and García-Saínz JA (2001) Angiotensin AT(1) receptor phosphorylation and desensitization in a hepatic cell line. Roles of protein kinase c and phosphoinositide 3-kinase. Mol Pharmacol 59 576-585.

Gardiner SM, Kemp PA, March JE, and Bennett T (1993) Regional haemodynamic effects of angiotensin II (3-8) in conscious rats. Br J Pharmacol 110:159-162.

Garg R and Yusuf S; Collaborative Group on ACE Inhibitor Trials (1995) Overview of randomized trials of angiotensin-converting enzyme inhibitors on mortality and morbidity in patients with heart failure. JAMA 273:1450-1456.

Gava E, de Castro CH, Ferreira AJ, Colleta H, Melo MB, Alenina N, Bader M, Oliveira LA, Santos RA, and Kitten GT (2012) Angiotensin-(1-7) receptor Mas is an essential modulator of extracellular matrix protein expression in the heart. Regul Pept 175:30-42.

Gembardt F, Grajewski S, Vahl M, Schultheiss HP, and Walther T (2008) Angiotensin metabolites can stimulate receptors of the Mas-related genes family. $\mathrm{Mol}$ Cell Biochem 319:115-123.

Gendron L, Laflamme L, Rivard N, Asselin C, Payet MD, and Gallo-Payet N (1999) Signals from the AT2 (angiotensin type 2) receptor of angiotensin II inhibit p21ras and activate MAPK (mitogen-activated protein kinase) to induce morphological neuronal differentiation in NG108-15 cells. Mol Endocrinol 13:1615-1626.

Gesualdo L, Ranieri E, Monno R, Rossiello MR, Colucci M, Semeraro N, Grandaliano G, Schena FP, Ursi M, and Cerullo G (1999) Angiotensin IV stimulates plasminogen 
activator inhibitor-1 expression in proximal tubular epithelial cells. Kidney Int $\mathbf{5 6}$ 461-470.

Giani JF, Burghi V, Veiras LC, Tomat A, Muñoz MC, Cao G, Turyn D, Toblli JE, and Dominici FP (2012) Angiotensin-(1-7) attenuates diabetic nephropathy in Zucker diabetic fatty rats. Am J Physiol Renal Physiol 302:F1606-F1615.

Giani JF, Muñoz MC, Mayer MA, Veiras LC, Arranz C, Taira CA, Turyn D, Toblli JE, and Dominici FP (2010) Angiotensin-(1-7) improves cardiac remodeling and inhibits growth-promoting pathways in the heart of fructose-fed rats. Am J Physiol Heart Circ Physiol 298:H1003-H1013.

Gingras B, Rodier G, Giasson E, Coulombe P, Chassagne C, and Meloche S (2003) Expression of angiotensin type II receptor downregulates Cdk4 synthesis and inhibits cell-cycle progression. Oncogene 22:2633-2642.

Gironacci MM, Adamo HP, Corradi G, Santos RA, Ortiz P, and Carretero OA (2011) Angiotensin (1-7) induces MAS receptor internalization. Hypertension 58:176-181.

Godeny MD, Sayyah J, VonDerLinden D, Johns M, Ostrov DA, Caldwell-Busby J, and Sayeski PP (2007) The N-terminal SH2 domain of the tyrosine phosphatase, SHP-2, is essential for Jak2-dependent signaling via the angiotensin II type AT1 receptor. Cell Signal 19:600-609.

Godin CM and Ferguson SS (2012) Biased agonism of the angiotensin II type 1 receptor. Mini Rev Med Chem 12:812-816.

Goette A, Arndt M, Röcken C, Spiess A, Staack T, Geller JC, Huth C, Ansorge S, Klein HU, and Lendeckel U (2000) Regulation of angiotensin II receptor subtypes during atrial fibrillation in humans. Circulation 101:2678-2681.

Gohlke P, Pees C, and Unger T (1998) AT2 receptor stimulation increases aortic cyclic GMP in SHRSP by a kinin-dependent mechanism. Hypertension 31:349-355

Goldblatt H, Lynch J, Hanzal RF, and Summerville WW (1934) Studies on Experimental Hypertension: I. The Production of Persistent Elevation of Systolic Blood Pressure by Means of Renal Ischemia. J Exp Med 59:347-379.

Goldfarb DA, Diz DI, Tubbs RR, Ferrario CM, and Novick AC (1994) Angiotensin II receptor subtypes in the human renal cortex and renal cell carcinoma. J Urol 151: 208-213.

Gomes ER, Lara AA, Almeida PW, Guimarães D, Resende RR, Campagnole-Santos MJ, Bader M, Santos RA, and Guatimosim S (2010) Angiotensin-(1-7) prevents cardiomyocyte pathological remodeling through a nitric oxide/guanosine $3^{\prime}, 5^{\prime}$-cyclic monophosphate-dependent pathway. Hypertension 55:153-160.

Gomes ER, Santos RA, and Guatimosim S (2012) Angiotensin-(1-7)-mediated signaling in cardiomyocytes. Int $J$ Hypertens 2012:493129.

González-Abraldes J, Albillos A, Bañares R, Del Arbol LR, Moitinho E, Rodríguez C, González M, Escorsell A, García-Pagán JC, and Bosch J (2001) Randomized comparison of long-term losartan versus propranolol in lowering portal pressure in cirrhosis. Gastroenterology 121:382-388.

Gossmann J, Burkhardt R, Harder S, Lenz T, Sedlmeyer A, Klinkhardt U, Geiger H, and Scheuermann EH (2001) Angiotensin II infusion increases plasma erythropoietin levels via an angiotensin II type 1 receptor-dependent pathway. Kidney Int 60:83-86.

Graham KA, Reaich D, Channon SM, Downie S, and Goodship TH (1997) Correction of acidosis in hemodialysis decreases whole-body protein degradation. J Am Soc Nephrol 8:632-637.

Gratton JP, Bernatchez P, and Sessa WC (2004) Caveolae and caveolins in the cardiovascular system. Circ Res 94:1408-1417.

Greco S, Muscella A, Elia MG, Salvatore P, Storelli C, and Marsigliante S (2002) Activation of angiotensin II type I receptor promotes protein kinase $\mathrm{C}$ translocation and cell proliferation in human cultured breast epithelial cells. $J$ Endocrinol 174: 205-214.

Gregg D, Rauscher FM, and Goldschmidt-Clermont PJ (2003) Rac regulates cardiovascular superoxide through diverse molecular interactions: more than a binary GTP switch. Am J Physiol Cell Physiol 285:C723-C734

Gribouval O, Gonzales M, Neuhaus T, Aziza J, Bieth E, Laurent N, Bouton JM, Feuillet F, Makni S, and Ben Amar H, et al. (2005) Mutations in genes in the reninangiotensin system are associated with autosomal recessive renal tubular dysgenesis. Nat Genet 37:964-968

Griendling KK, Delafontaine P, Rittenhouse SE, Gimbrone MA Jr, and Alexander RW (1987) Correlation of receptor sequestration with sustained diacylglycerol accumulation in angiotensin II-stimulated cultured vascular smooth muscle cells. $J$ Biol Chem 262:14555-14562.

Griendling KK, Lassègue B, and Alexander RW (1996) Angiotensin receptors and their therapeutic implications. Annu Rev Pharmacol Toxicol 36:281-306.

Griendling KK, Sorescu D, and Ushio-Fukai M (2000) NAD $(\mathrm{P}) \mathrm{H}$ oxidase: role in cardiovascular biology and disease. Circ Res 86:494-501.

Griffin SA, Brown WC, MacPherson F, McGrath JC, Wilson VG, Korsgaard N, Mulvany MJ, and Lever AF (1991) Angiotensin II causes vascular hypertrophy in part by a non-pressor mechanism. Hypertension 17:626-635.

Grobe JL and Katovich MJ (2006) Alterations in aortic vascular reactivity to angiotensin 1-7 in 17-beta-estradiol-treated female SD rats. Regul Pept 133:62-67.

Groblewski T, Maigret B, Larguier R, Lombard C, Bonnafous JC, and Marie J (1997) Mutation of Asn111 in the third transmembrane domain of the AT1A angiotensin II receptor induces its constitutive activation. J Biol Chem 272:1822-1826.

Gross V, Schunck WH, Honeck H, Milia AF, Kärgel E, Walther T, Bader M, Inagami $\mathrm{T}$, Schneider W, and Luft FC (2000) Inhibition of pressure natriuresis in mice lacking the AT2 receptor. Kidney Int 57:191-202.

Gryglewski RJ, Palmer RM, and Moncada S (1986) Superoxide anion is involved in the breakdown of endothelium-derived vascular relaxing factor. Nature 320: $454-456$.

Gschwind A, Zwick E, Prenzel N, Leserer M, and Ullrich A (2001) Cell communication networks: epidermal growth factor receptor transactivation as the paradigm for interreceptor signal transmission. Oncogene 20:1594-1600.

Guang C, Phillips RD, Jiang B, and Milani F (2012) Three key proteasesangiotensin-I-converting enzyme (ACE), ACE2 and renin-within and beyond the renin-angiotensin system. Arch Cardiovasc Dis 105:373-385.
Guillemot L, Levy A, Raymondjean M, and Rothhut B (2001) Angiotensin II-induced transcriptional activation of the cyclin D1 gene is mediated by Egr-1 in CHO-AT (1A) cells. J Biol Chem 276:39394-39403.

Guillemot L, Levy A, Zhao ZJ, Bereziat G, and Rothhut B (2000) The protein-tyrosine phosphatase SHP-2 is required during angiotensin II-mediated activation of cyclin D1 promoter in CHO-AT1A cells. J Biol Chem 275:26349-26358.

Guimaraes PS, Santiago NM, Xavier CH, Velloso EP, Fontes MA, Santos RA and Campagnole-Santos MJ (2012) Chronic infusion of angiotensin-(1-7) into the lateral ventricle of the brain attenuates hypertension in DOCA-salt rats. Am J Physiol Heart Circ Physiol 303:H393-H400.

Guimond MO and Gallo-Payet N (2012) The Angiotensin II Type 2 Receptor in Brain Functions: An Update. Int $J$ Hypertens 2012:351758.

Gunther S, Gimbrone MA Jr, and Alexander RW (1980) Regulation by angiotensin II of its receptors in resistance blood vessels. Nature 287:230-232.

Guo DF, Chenier I, Tardif V, Orlov SN, and Inagami T (2003) Type 1 angiotensin II receptor-associated protein ARAP1 binds and recycles the receptor to the plasma membrane. Biochem Biophys Res Commun 310:1254-1265.

Guo DF, Sun YL, Hamet P, and Inagami T (2001) The angiotensin II type 1 receptor and receptor-associated proteins. Cell Res 11:165-180.

Guo S, Lopez-Ilasaca M, and Dzau VJ (2005) Identification of calcium-modulating cyclophilin ligand (CAML) as transducer of angiotensin II-mediated nuclear factor of activated T cells (NFAT) activation. J Biol Chem 280:12536-12541.

Gutkind JS (1998) The pathways connecting G protein-coupled receptors to the nucleus through divergent mitogen-activated protein kinase cascades. J Biol Chem 273:1839-1842.

Gwathmey TM, Alzayadneh EM, Pendergrass KD, and Chappell MC (2012) Novel roles of nuclear angiotensin receptors and signaling mechanisms. Am J Physiol Regul Integr Comp Physiol 302:R518-R530.

Habiyakare B, Alsaadon H, Mathai ML, Hayes A, and Zulli A (2014) Reduction of angiotensin $\mathrm{A}$ and alamandine vasoactivity in the rabbit model of atherogenesis: differential effects of alamandine and Ang(1-7). Int J Exp Pathol 95:290-295.

Hall JE, Mizelle HL, Brands MW, and Hildebrandt DA (1992) Pressure natriuresis and angiotensin II in reduced kidney mass, salt-induced hypertension. Am J Physiol 262:R61-R71.

Hall KL, Hanesworth JM, Ball AE, Felgenhauer GP, Hosick HL, and Harding JW (1993) Identification and characterization of a novel angiotensin binding site in cultured vascular smooth muscle cells that is specific for the hexapeptide (3-8) fragment of angiotensin II, angiotensin IV. Regul Pept 44:225-232.

Hamilton TA, Handa RK, Harding JW, and Wright JW (2001) A role for the angiotensin IV/AT4 system in mediating natriuresis in the rat. Peptides 22:935-944.

Han HM, Shimuta SI, Kanashiro CA, Oliveira L, Han SW, and Paiva AC (1998 Residues Val254, His256, and Phe259 of the angiotensin II AT1 receptor are not involved in ligand binding but participate in signal transduction. Mol Endocrinol 12:810-814.

Han L, Ma C, Liu Q, Weng HJ, Cui Y, Tang Z, Kim Y, Nie H, Qu L, and Patel KN, et al. (2013) A subpopulation of nociceptors specifically linked to itch. Nat Neurosci 16:174-182.

Handa RK (2001) Characterization and signaling of the AT(4) receptor in human proximal tubule epithelial (HK-2) cells. J Am Soc Nephrol 12:440-449.

Handa RK, Krebs LT, Harding JW, and Handa SE (1998) Angiotensin IV AT4receptor system in the rat kidney. Am $J$ Physiol 274:F290-F299.

Hanesworth JM, Sardinia MF, Krebs LT, Hall KL, and Harding JW (1993) Elucidation of a specific binding site for angiotensin II(3-8), angiotensin IV, in mammalian heart membranes. J Pharmacol Exp Ther 266:1036-1042.

Hansen JL, Aplin M, Hansen JT, Christensen GL, Bonde MM, Schneider M, Hauns $\varnothing$ $\mathrm{S}$, Schiffer HH, Burstein ES, and Weiner DM, et al. (2008) The human angiotensin AT(1) receptor supports G protein-independent extracellular signal-regulated kinase 1/2 activation and cellular proliferation. Eur J Pharmacol 590:255-263.

Hansen JL, Theilade J, Hauns $ø$ S, and Sheikh SP (2004) Oligomerization of wild type and nonfunctional mutant angiotensin II type I receptors inhibits galphaq protein signaling but not ERK activation. $J$ Biol Chem 279:24108-24115.

Hao J, Wang B, Jones SC, Jassal DS, and Dixon IM (2000) Interaction between angiotensin II and Smad proteins in fibroblasts in failing heart and in vitro. Am J Physiol Heart Circ Physiol 279:H3020-H3030.

Harada K, Sugaya T, Murakami K, Yazaki Y, and Komuro I (1999) Angiotensin II type $1 \mathrm{~A}$ receptor knockout mice display less left ventricular remodeling and improved survival after myocardial infarction. Circulation 100:2093-2099.

Harding JW, Cook VI, Miller-Wing AV, Hanesworth JM, Sardinia MF, Hall KL, Stobb JW, Swanson GN, Coleman JK, and Wright JW, et al. (1992) Identification of an AII(3-8) [AIV] binding site in guinea pig hippocampus. Brain Res 583:340-343.

Harding JW, Wright JW, Swanson GN, Hanesworth JM, and Krebs LT (1994) AT4 receptors: specificity and distribution. Kidney Int 46:1510-1512.

Harris RC (2012) Podocyte ACE2 protects against diabetic nephropathy. Kidney Int 82:255-256.

Hayashida W, Horiuchi M, and Dzau VJ (1996) Intracellular third loop domain of angiotensin II type-2 receptor. Role in mediating signal transduction and cellular function. J Biol Chem 271:21985-21992.

He JG, Huang YY, Ma H, He XH, Liao XX, Wang LC, and Zhou JZ (2004) [Effects of angiotensin-(1-7) on remodeling of myocardial collagen network in pressureoverloaded rats]. Zhongguo Wei Zhong Bing Ji Jiu Yi Xue 16:523-526.

Healy DP and Wilk S (1993) Localization of immunoreactive glutamyl aminopeptidase in rat brain. II. Distribution and correlation with angiotensin II. Brain Res 606:295-303.

Heeneman S, Haendeler J, Saito Y, Ishida M, and Berk BC (2000) Angiotensin II induces transactivation of two different populations of the platelet-derived growth factor beta receptor. Key role for the p66 adaptor protein Shc. J Biol Chem 275: 15926-15932.

Heimfeld S, Fogarty B, McGuire K, Williams S, and Berenson RJ (1992) Peripheral blood stem cell mobilization after stem cell factor or G-CSF treatment: rapid 
enrichment for stem and progenitor cells using the CEPRATE immunoaffinity separation system. Transplant Proc 24:2818.

Hein L, Barsh GS, Pratt RE, Dzau VJ, and Kobilka BK (1995) Behavioural and cardiovascular effects of disrupting the angiotensin II type-2 receptor in mice. Nature 377:744-747.

Hein L, Stevens ME, Barsh GS, Pratt RE, Kobilka BK, and Dzau VJ (1997) Overexpression of angiotensin AT1 receptor transgene in the mouse myocardium produces a lethal phenotype associated with myocyte hyperplasia and heart block. Proc Natl Acad Sci USA 94:6391-6396.

Heineke J and Molkentin JD (2006) Regulation of cardiac hypertrophy by intracellular signalling pathways. Nat Rev Mol Cell Biol 7:589-600.

Henriksen EJ, Jacob S, Kinnick TR, Teachey MK, and Krekler M (2001) Selective angiotensin II receptor antagonism reduces insulin resistance in obese Zucker rats. Hypertension 38:884-890.

Herbst JJ, Ross SA, Scott HM, Bobin SA, Morris NJ, Lienhard GE, and Keller SR (1997) Insulin stimulates cell surface aminopeptidase activity toward vasopressin in adipocytes. Am J Physiol 272:E600-E606.

Heringer-Walther S, Gembardt F, Perschel FH, Katz N, Schultheiss HP, and Walther $\mathrm{T}$ (2012) The genetic deletion of Mas abolishes salt induced hypertension in mice. Eur J Pharmacol 689:147-153.

Herradon G, Ezquerra L, Nguyen T, Vogt TF, Bronson R, Silos-Santiago I, and Deuel TF (2004) Pleiotrophin is an important regulator of the renin-angiotensin system in mouse aorta. Biochem Biophys Res Commun 324:1041-1047.

Herse F and LaMarca B (2013) Angiotensin II type 1 receptor autoantibody (AT1-AA)-mediated pregnancy hypertension. Am J Reprod Immunol 69:413-418.

Herse F, Staff AC, Hering L, Müller DN, Luft FC, and Dechend R (2008) AT1receptor autoantibodies and uteroplacental RAS in pregnancy and pre-eclampsia. $J$ Mol Med (Berl) 86:697-703

Higuchi S, Ohtsu H, Suzuki H, Shirai H, Frank GD, and Eguchi S (2007) Angiotensin II signal transduction through the AT1 receptor: novel insights into mechanisms and pathophysiology. Clin Sci (Lond) 112:417-428.

Hirose A, Ono M, Saibara T, Nozaki Y, Masuda K, Yoshioka A, Takahashi M, Akisawa N, Iwasaki S, and Oben JA, et al. (2007) Angiotensin II type 1 receptor blocker inhibits fibrosis in rat nonalcoholic steatohepatitis. Hepatology $\mathbf{4 5}$ 1375-1381.

Hjorth SA, Schambye HT, Greenlee WJ, and Schwartz TW (1994) Identification of peptide binding residues in the extracellular domains of the AT1 receptor. J Biol Chem 269:30953-30959.

Hoffmann S, Krause T, van Geel PP, Willenbrock R, Pagel I, Pinto YM, Buikema H, van Gilst WH, Lindschau C, and Paul M, et al. (2001) Overexpression of the human angiotensin II type 1 receptor in the rat heart augments load induced cardiac hypertrophy. J Mol Med (Berl) 79:601-608.

Hoffmann S, Podlich D, Hähnel B, Kriz W, and Gretz N (2004) Angiotensin II type 1 receptor overexpression in podocytes induces glomerulosclerosis in transgenic rats J Am Soc Nephrol 15:1475-1487.

Hohenfellner K, Hunley TE, Schloemer C, Brenner W, Yerkes E, Zepp F, Brock JW 3rd, and Kon V (1999) Angiotensin type 2 receptor is important in the normal development of the ureter. Pediatr Nephrol 13:187-191.

Höhle S, Spitznagel H, Rascher W, Culman J, and Unger T (1995) Angiotensin AT1 receptor-mediated vasopressin release and drinking are potentiated by an AT2 receptor antagonist. Eur J Pharmacol 275:277-282.

Holloway AC, Qian H, Pipolo L, Ziogas J, Miura S, Karnik S, Southwell BR, Lew MJ, and Thomas WG (2002) Side-chain substitutions within angiotensin II reveal different requirements for signaling, internalization, and phosphorylation of type $1 \mathrm{~A}$ angiotensin receptors. Mol Pharmacol 61:768-777.

Honorato-Sampaio K, Pereira VM, Santos RA, and Reis AM (2012) Evidence that angiotensin-(1-7) is an intermediate of gonadotrophin-induced oocyte maturation in the rat preovulatory follicle. Exp Physiol 97:642-650.

Horiuchi M, Hayashida W, Kambe T, Yamada T, and Dzau VJ (1997) Angiotensin type 2 receptor dephosphorylates Bcl-2 by activating mitogen-activated protein kinase phosphatase-1 and induces apoptosis. J Biol Chem 272:19022-19026.

Horiuchi M, Iwanami J, and Mogi M (2012) Regulation of angiotensin II receptors beyond the classical pathway. Clin Sci (Lond) 123:193-203.

Horiuchi M, Lehtonen JY, and Daviet L (1999) Signaling Mechanism of the AT2 Angiotensin II Receptor: Crosstalk between AT1 and AT2 Receptors in Cell Growth. Trends Endocrinol Metab 10:391-396.

Hosaka T, Brooks CC, Presman E, Kim SK, Zhang Z, Breen M, Gross DN, Sztul E, and Pilch PF (2005) p115 Interacts with the GLUT4 vesicle protein, IRAP, and plays a critical role in insulin-stimulated GLUT4 translocation. Mol Biol Cell 16: 2882-2890.

Hou M, Pantev E, Möller S, Erlinge D, and Edvinsson L (2000) Angiotensin II type 1 receptors stimulate protein synthesis in human cardiac fibroblasts via a $\mathrm{Ca} 2$ +-sensitive PKC-dependent tyrosine kinase pathway. Acta Physiol Scand 168 301-309.

Huang XC, Richards EM, and Sumners C (1995) Angiotensin II type 2 receptormediated stimulation of protein phosphatase $2 \mathrm{~A}$ in rat hypothalamic/brainstem neuronal cocultures. $J$ Neurochem 65:2131-2137.

Hubel CA (1999) Oxidative stress in the pathogenesis of preeclampsia. Proc Soc Exp Biol Med 222:222-235.

Humphrey PP and Barnard EA (1998) International Union of Pharmacology. XIX. The IUPHAR receptor code: a proposal for an alphanumeric classification system. Pharmacol Rev 50:271-277.

Hunton DL, Barnes WG, Kim J, Ren XR, Violin JD, Reiter E, Milligan G, Patel DD, and Lefkowitz RJ (2005) Beta-arrestin 2-dependent angiotensin II type 1A receptor-mediated pathway of chemotaxis. Mol Pharmacol 67:1229-1236.

Hunyady L (2009) Physiological and pathological actions of the renin-angiotensin system. Mol Cell Endocrinol 302:109-110.

Hunyady L, Balla T, and Catt KJ (1996) The ligand binding site of the angiotensin AT1 receptor. Trends Pharmacol Sci 17:135-140.
Hunyady L, Baukal AJ, Gaborik Z, Olivares-Reyes JA, Bor M, Szaszak M, Lodge R, Catt KJ, and Balla T (2002) Differential PI 3-kinase dependence of early and late phases of recycling of the internalized AT1 angiotensin receptor. J Cell Biol 157: 1211-1222.

Hunyady L, Bor M, Balla T, and Catt KJ (1994) Identification of a cytoplasmic SerThr-Leu motif that determines agonist-induced internalization of the AT1 angiotensin receptor. J Biol Chem 269:31378-31382.

Hunyady L and Catt KJ (2006) Pleiotropic AT1 receptor signaling pathways mediating physiological and pathogenic actions of angiotensin II. Mol Endocrinol 20 953-970.

Hunyady L, Catt KJ, Clark AJ, and Gáborik Z (2000) Mechanisms and functions of AT(1) angiotensin receptor internalization. Regul Pept 91:29-44

Hunyady L, Ji H, Jagadeesh G, Zhang M, Gáborik Z, Mihalik B, and Catt KJ (1998) Dependence of AT1 angiotensin receptor function on adjacent asparagine residues in the seventh transmembrane helix. Mol Pharmacol 54:427-434.

Hunyady L and Turu G (2004) The role of the AT1 angiotensin receptor in cardiac hypertrophy: angiotensin II receptor or stretch sensor? Trends Endocrinol Metab 15:405-408.

Huynh J, Thomas WG, Aguilar MI, and Pattenden LK (2009) Role of helix 8 in G protein-coupled receptors based on structure-function studies on the type 1 angiotensin receptor. Mol Cell Endocrinol 302:118-127.

Iafrate AJ, Feuk L, Rivera MN, Listewnik ML, Donahoe PK, Qi Y, Scherer SW, and Lee C (2004) Detection of large-scale variation in the human genome. Nat Genet 36:949-951.

Ichihara S, Senbonmatsu T, Price E Jr, Ichiki T, Gaffney FA, and Inagami T (2001) Angiotensin II type 2 receptor is essential for left ventricular hypertrophy and cardiac fibrosis in chronic angiotensin II-induced hypertension. Circulation 104: $346-351$.

Ichiki T and Inagami T (1995) Expression, genomic organization, and transcription of the mouse angiotensin II type 2 receptor gene. Circ Res 76:693-700.

Ichiki T, Labosky PA, Shiota C, Okuyama S, Imagawa Y, Fogo A, Niimura F, Ichikawa I, Hogan BL, and Inagami T (1995) Effects on blood pressure and exploratory behaviour of mice lacking angiotensin II type-2 receptor. Nature 377: $748-750$

Igarashi M, Hirata A, Yamaguchi H, Tsuchiya H, Ohnuma H, Tominaga M, Daimon M, and Kato T (2001) Candesartan inhibits carotid intimal thickening and ameliorates insulin resistance in balloon-injured diabetic rats. Hypertension 38 $1255-1259$

Inagami T, Iwai N, Sasaki K, Guo DF, Furuta H, Yamano Y, Bardhan S, Chaki S Makito N, and Badr K (1993) Angiotensin II receptors: cloning and regulation. Arzneimittelforschung 43 (2A):226-228.

Ino K, Shibata K, Yamamoto E, Kajiyama H, Nawa A, Mabuchi Y, Yagi S, Minami S, Tanizaki Y, and Kobayashi A, et al. (2011) Role of the renin-angiotensin system in gynecologic cancers. Curr Cancer Drug Targets 11:405-411.

Ino K, Uehara C, Kikkawa F, Kajiyama H, Shibata K, Suzuki T, Khin EE, Ito M, Takeuchi M, and Itakura A, et al. (2003) Enhancement of aminopeptidase A expression during angiotensin II-induced choriocarcinoma cell proliferation through AT1 receptor involving protein kinase C- and mitogen-activated protein kinasedependent signaling pathway. J Clin Endocrinol Metab 88:3973-3982.

Inokuchi S, Kimura K, Sugaya T, Inokuchi K, Murakami K, and Sakai T (2001) Hyperplastic vascular smooth muscle cells of the intrarenal arteries in angiotensin II type 1a receptor null mutant mice. Kidney Int 60:722-731.

Inoue Y, Nakamura N, and Inagami T (1997) A review of mutagenesis studies of angiotensin II type 1 receptor, the three-dimensional receptor model in search of the agonist and antagonist binding site and the hypothesis of a receptor activation mechanism. J Hypertens 15:703-714

Iozzo RV (1998) Matrix proteoglycans: from molecular design to cellular function. Annu Rev Biochem 67:609-652.

Ishida M, Ishida T, Thomas SM, and Berk BC (1998) Activation of extracellular signal-regulated kinases (ERK1/2) by angiotensin II is dependent on c-Src in vascular smooth muscle cells. Circ Res 82:7-12.

Ishida M, Marrero MB, Schieffer B, Ishida T, Bernstein KE, and Berk BC (1995) Angiotensin II activates pp60c-src in vascular smooth muscle cells. Circ Res $\mathbf{7 7}$ 1053-1059

Ishida T, Ishida M, Suero J, Takahashi M, and Berk BC (1999) Agonist-stimulated cytoskeletal reorganization and signal transduction at focal adhesions in vascular smooth muscle cells require c-Src. J Clin Invest 103:789-797.

Ishizaka N, Alexander RW, Laursen JB, Kai H, Fukui T, Oppermann M, Lefkowitz RJ, Lyons PR, and Griendling KK (1997) G protein-coupled receptor kinase 5 in cultured vascular smooth muscle cells and rat aorta. Regulation by angiotensin II and hypertension. $J$ Biol Chem 272:32482-32488.

Ishizaka N, Griendling KK, Lassègue B, and Alexander RW (1998) Angiotensin II type 1 receptor: relationship with caveolae and caveolin after initial agonist stimulation. Hypertension 32:459-466.

Ito M, Oliverio MI, Mannon PJ, Best CF, Maeda N, Smithies O, and Coffman TM (1995) Regulation of blood pressure by the type 1A angiotensin II receptor gene. Proc Natl Acad Sci USA 92:3521-3525.

Iusuf D, Henning RH, van Gilst WH, and Roks AJ (2008) Angiotensin-(1-7): pharmacological properties and pharmacotherapeutic perspectives. Eur $J$ Pharmacol 585:303-312.

Iwai M, Chen R, Li Z, Shiuchi T, Suzuki J, Ide A, Tsuda M, Okumura M, Min LJ, and Mogi M, et al. (2005) Deletion of angiotensin II type 2 receptor exaggerated atherosclerosis in apolipoprotein E-null mice. Circulation 112:1636-1643.

Iwanciw D, Rehm M, Porst M, and Goppelt-Struebe M (2003) Induction of connective tissue growth factor by angiotensin II: integration of signaling pathways. Arterioscler Thromb Vasc Biol 23:1782-1787.

Iwata M, Cowling RT, Gurantz D, Moore C, Zhang S, Yuan JX, and Greenberg BH (2005) Angiotensin-(1-7) binds to specific receptors on cardiac fibroblasts to initiate antifibrotic and antitrophic effects. Am J Physiol Heart Circ Physiol 289: $\mathrm{H} 2356-\mathrm{H} 2363$ 
Iyer SN, Chappell MC, Averill DB, Diz DI, and Ferrario CM (1998) Vasodepressor actions of angiotensin-(1-7) unmasked during combined treatment with lisinopril and losartan. Hypertension 31:699-705.

Jackson TR, Blair LA, Marshall J, Goedert M, and Hanley MR (1988) The mas oncogene encodes an angiotensin receptor. Nature 335:437-440.

Jackson TR and Hanley MR (1989) Tumor promoter 12-O-tetradecanoylphorbol 13-acetate inhibits mas/angiotensin receptor-stimulated inositol phosphate production and intracellular Ca2+ elevation in the 401L-C3 neuronal cell line. FEBS Lett 251:27-30.

Jankowski V, Tolle M, Santos RA, Gunthner T, Krause E, Beyermann M, Welker P, Bader M, Pinheiro SV, and Sampaio WO, et al. (2011) Angioprotectin: an angiotensin II-like peptide causing vasodilatory effects. FASEB J 25:2987-2995.

Janssen JW, Steenvoorden AC, Schmidtberger M, and Bartram CR (1988) Activation of the mas oncogene during transfection of monoblastic cell line DNA. Leukemia 2 : $318-320$.

Jarajapu YP, Bhatwadekar AD, Caballero S, Hazra S, Shenoy V, Medina R, Kent D, Stitt AW, Thut C, and Finney EM, et al. (2013) Activation of the ACE2/angiotensin(1-7)/Mas receptor axis enhances the reparative function of dysfunctional diabetic endothelial progenitors. Diabetes 62:1258-1269.

Jarvis MF, Gessner GW, and Ly CQ (1992) The angiotensin hexapeptide 3-8 fragment potently inhibits [125I] angiotensin II binding to non-AT1 or -AT2 recognition sites in bovine adrenal cortex. Eur J Pharmacol 219:319-322.

Jethon A, Pula B, Piotrowska A, Wojnar A, Rys J, Dziegiel P, and Podhorska-Okolow M (2012) Angiotensin II type 1 receptor (AT-1R) expression correlates with VEGFA and VEGF-D expression in invasive ductal breast cancer. Pathol Oncol Res 18: 867-873.

Jewett ME, Kronauer RE, and Czeisler CA (1991) Light-induced suppression of endogenous circadian amplitude in humans. Nature 350:59-62.

Ji H, Leung M, Zhang Y, Catt KJ, and Sandberg K (1994) Differential structural requirements for specific binding of nonpeptide and peptide antagonists to the AT1 angiotensin receptor. Identification of amino acid residues that determine binding of the antihypertensive drug losartan. J Biol Chem 269:16533-16536.

Jiang F, Yang J, Zhang Y, Dong M, Wang S, Zhang Q, Liu FF, Zhang K, and Zhang C (2014) Angiotensin-converting enzyme 2 and angiotensin 1-7: novel therapeutic targets. Nat Rev Cardiol 11:413-426.

Jin XQ, Fukuda N, Su JZ, Lai YM, Suzuki R, Tahira Y, Takagi H, Ikeda Y, Kanmatsuse K, and Miyazaki H (2002) Angiotensin II type 2 receptor gene transfer downregulates angiotensin II type 1a receptor in vascular smooth muscle cells. Hypertension 39:1021-1027.

Jöhren O and Saavedra JM (1996) Expression of AT1A and AT1B angiotensin II receptor messenger RNA in forebrain of 2-wk-old rats. Am $J$ Physiol 271: E104-E112.

Jones ES, Del Borgo MP, Kirsch JF, Clayton D, Bosnyak S, Welungoda I, Hausler N, Unabia S, Perlmutter P, and Thomas WG, et al. (2011) A single beta-amino acid substitution to angiotensin II confers AT2 receptor selectivity and vascular function. Hypertension 57:570-576.

Joseph MP, Maigret B, Bonnafous JC, Marie J, and Scheraga HA (1995) A computer modeling postulated mechanism for angiotensin II receptor activation. J Protein Chem 14:381-398.

Ju H and Dixon IM (1996) Extracellular matrix and cardiovascular diseases. Can $J$ Cardiol 12:1259-1267.

Kagiyama S, Varela A, Phillips MI, and Galli SM (2001) Antisense inhibition of brain renin-angiotensin system decreased blood pressure in chronic 2-kidney, 1 clip hypertensive rats. Hypertension 37:371-375

Kakar SS, Sellers JC, Devor DC, Musgrove LC, and Neill JD (1992) Angiotensin II type- 1 receptor subtype cDNAs: differential tissue expression and hormonal regulation. Biochem Biophys Res Commun 183:1090-1096.

Kakuchi J, Ichiki T, Kiyama S, Hogan BL, Fogo A, Inagami T, and Ichikawa I (1995) Developmental expression of renal angiotensin II receptor genes in the mouse. Kidney Int 47:140-147.

Kakuta H, Sudoh K, Sasamata M, and Yamagishi S (2005) Telmisartan has the strongest binding affinity to angiotensin II type 1 receptor: comparison with other angiotensin II type 1 receptor blockers. Int J Clin Pharmacol Res 25:41-46.

Kalinowski L, Matys T, Chabielska E, Buczko W, and Malinski T (2002) Angiotensin II AT1 receptor antagonists inhibit platelet adhesion and aggregation by nitric oxide release. Hypertension 40:521-527.

Kambayashi Y, Bardhan S, Takahashi K, Tsuzuki S, Inui H, Hamakubo T, and Inagami T (1993) Molecular cloning of a novel angiotensin II receptor isoform involved in phosphotyrosine phosphatase inhibition. J Biol Chem 268: $24543-24546$.

Kambayashi Y, Nagata K, Ichiki T, and Inagami T (1996) Insulin and insulin-like growth factors induce expression of angiotensin type-2 receptor in vascularsmooth-muscle cells. Eur J Biochem 239:558-565.

Kambayashi Y, Takahashi K, Bardhan S, and Inagami T (1994) Molecular structure and function of angiotensin type 2 receptor. Kidney Int 46:1502-1504.

Kandror KV and Pilch PF (1994) gp160, a tissue-specific marker for insulin-activated glucose transport. Proc Natl Acad Sci USA 91:8017-8021.

Kandror KV, Yu L, and Pilch PF (1994) The major protein of GLUT4-containing vesicles, gp160, has aminopeptidase activity. J Biol Chem 269:30777-30780.

Kang J, Posner P, and Sumners C (1994) Angiotensin II type 2 receptor stimulation of neuronal $\mathrm{K}+$ currents involves an inhibitory GTP binding protein. Am J Physiol 267:C1389-C1397.

Kang J, Sumners C, and Posner P (1993) Angiotensin II type 2 receptor-modulated changes in potassium currents in cultured neurons. Am J Physiol 265:C607-C616.

Kar S, Gao L, Belatti DA, Curry PL, and Zucker IH (2011) Central angiotensin (1-7) enhances baroreflex gain in conscious rabbits with heart failure. Hypertension $\mathbf{5 8}$ $627-634$.

Karip E, Turu G, Süpeki K, Szidonya L, and Hunyady L (2007) Cross-inhibition of angiotensin AT1 receptors supports the concept of receptor oligomerization. Neurochem Int 51:261-267.
Karnik S, Unal H, Kemp JR, Tirupula K, Thomas WG, Eguchi S and Vanderheyden P (2014) Angiotensin receptors, introduction. IUPHAR/BPS Guide to PHARMACOLOGY, http://www.guidetopharmacology.org/GRAC/ FamilyIntroductionForward? familyId $=6$

Karnik SS, Gogonea C, Patil S, Saad Y, and Takezako T (2003) Activation of G-protein-coupled receptors: a common molecular mechanism. Trends Endocrinol Metab 14:431-437.

Karnik SS, Husain A, and Graham RM (1996) Molecular determinants of peptide and non-peptide binding to the AT1 receptor. Clin Exp Pharmacol Physiol Suppl 3 S58-S66.

Kaschina E, Grzesiak A, Li J, Foryst-Ludwig A, Timm M, Rompe F, Sommerfeld M Kemnitz UR, Curato C, and Namsolleck P, et al. (2008) Angiotensin II type 2 receptor stimulation: a novel option of therapeutic interference with the reninangiotensin system in myocardial infarction? Circulation 118:2523-2532.

Kaschina E and Unger T (2003) Angiotensin AT1/AT2 receptors: regulation, signalling and function. Blood Press 12:70-88.

Kashem SW, Subramanian H, Collington SJ, Magotti P, Lambris JD, and Ali H (2011) G protein coupled receptor specificity for C3a and compound 48/80-induced degranulation in human mast cells: roles of Mas-related genes MrgX1 and MrgX2. Eur J Pharmacol 668:299-304.

Kato H, Suzuki H, Tajima S, Ogata Y, Tominaga T, Sato A, and Saruta T (1991) Angiotensin II stimulates collagen synthesis in cultured vascular smooth muscle cells. J Hypertens 9:17-22.

Kato J, Koda M, Kishina M, Tokunaga S, Matono T, Sugihara T, Ueki M, and Murawaki Y (2012) Therapeutic effects of angiotensin II type 1 receptor blocker, irbesartan, on non-alcoholic steatohepatitis using FLS-ob/ob male mice. Int $J$ Mol Med 30:107-113.

Kaufman JS, Owoaje EE, Rotimi CN, and Cooper RS (1999) Blood pressure change in Africa: case study from Nigeria. Hum Biol 71:641-657.

Kawano H, Do YS, Kawano Y, Starnes V, Barr M, Law RE, and Hsueh WA (2000) Angiotensin II has multiple profibrotic effects in human cardiac fibroblasts. Circulation 101:1130-1137.

Kawano S, Kubota T, Monden Y, Kawamura N, Tsutsui H, Takeshita A, and Sunagawa K (2005) Blockade of NF-kappaB ameliorates myocardial hypertrophy in response to chronic infusion of angiotensin II. Cardiovasc Res 67: 689-698.

Keidar S, Attias J, Smith J, Breslow JL, and Hayek T (1997) The angiotensin-II receptor antagonist, losartan, inhibits LDL lipid peroxidation and atherosclerosis in apolipoprotein E-deficient mice. Biochem Biophys Res Commun 236:622-625.

Keiser JA, Bjork FA, Hodges JC, and Taylor DG Jr (1992) Renal hemodynamic and excretory responses to PD 123319 and losartan, nonpeptide AT1 and AT2 subtypespecific angiotensin II ligands. J Pharmacol Exp Ther 262:1154-1160.

Keller SR, Scott HM, Mastick CC, Aebersold R, and Lienhard GE (1995) Cloning and characterization of a novel insulin-regulated membrane aminopeptidase from Glut4 vesicles. J Biol Chem 270:23612-23618.

Kemp BA, Howell NL, Gildea JJ, Keller SR, Padia SH, and Carey RM (2014a) $\mathrm{AT}_{2}$ receptor activation induces natriuresis and lowers blood pressure. Circ Res 115 388-399.

Kemp JR, Unal H, Desnoyer R, Yue H, Bhatnagar A, and Karnik SS (2014b) Angiotensin II-regulated microRNA 483-3p directly targets multiple components of the renin-angiotensin system. J Mol Cell Cardiol 75:25-39.

Kenakin T (2001) Inverse, protean, and ligand-selective agonism: matters of receptor conformation. FASEB J 15:598-611.

Kenakin T (2003) Ligand-selective receptor conformations revisited: the promise and the problem. Trends Pharmacol Sci 24:346-354.

Kenakin T (2005) New concepts in drug discovery: collateral efficacy and permissive antagonism. Nat Rev Drug Discov 4:919-927.

Kenakin T (2007) Functional selectivity through protean and biased agonism: who steers the ship? Mol Pharmacol 72:1393-1401.

Kendall RT, Strungs EG, Rachidi SM, Lee MH, El-Shewy HM, Luttrell DK, Janech MG, and Luttrell LM (2011) The beta-arrestin pathway-selective type 1A angiotensin receptor (AT1A) agonist [Sar1,Ile4,Ile8] angiotensin II regulates a robust G protein-independent signaling network. J Biol Chem 286:19880-19891.

Khosla MC, Hall MM, Smeby RR, and Bumpus FM (1974) Synthesis of analogs of (8-isoleucine)angiotensin II with variations in position 1. J Med Chem 17:431-433.

Kijima K, Matsubara H, Murasawa S, Maruyama K, Mori Y, Ohkubo N, Komuro I, Yazaki Y, Iwasaka T, and Inada M (1996) Mechanical stretch induces enhanced expression of angiotensin II receptor subtypes in neonatal rat cardiac myocytes. Circ Res 79:887-897.

Kim J, Ahn S, Rajagopal K, and Lefkowitz RJ (2009) Independent beta-arrestin2 and $\mathrm{Gq} /$ protein kinase Czeta pathways for ERK stimulated by angiotensin type 1A receptors in vascular smooth muscle cells converge on transactivation of the epidermal growth factor receptor. J Biol Chem 284:11953-11962.

Kim J, Ahn S, Ren XR, Whalen EJ, Reiter E, Wei H, and Lefkowitz RJ (2005a) Functional antagonism of different $\mathrm{G}$ protein-coupled receptor kinases for beta-arrestinmediated angiotensin II receptor signaling. Proc Natl Acad Sci USA 102:1442-1447.

Kim NH, Ahn Y, Oh SK, Cho JK, Park HW, Kim YS, Hong MH, Nam KI, Park WJ, and Jeong $\mathrm{MH}$, et al. (2005b) Altered patterns of gene expression in response to chronic atrial fibrillation. Int Heart $J$ 46:383-395.

Kim S and Iwao H (2000) Molecular and cellular mechanisms of angiotensin IImediated cardiovascular and renal diseases. Pharmacol Rev 52:11-34.

Kim S, Izumi Y, Yano M, Hamaguchi A, Miura K, Yamanaka S, Miyazaki H, and Iwao H (1998) Angiotensin blockade inhibits activation of mitogen-activated protein kinases in rat balloon-injured artery. Circulation 97:1731-1737.

Kim S, Ohta K, Hamaguchi A, Yukimura T, Miura K, and Iwao H (1995) Angiotensin II induces cardiac phenotypic modulation and remodeling in vivo in rats. Hypertension 25:1252-1259.

Kim S, Zhan Y, Izumi Y, Yasumoto H, Yano M, and Iwao H (2000) In vivo activation of rat aortic platelet-derived growth factor and epidermal growth factor receptors by angiotensin II and hypertension. Arterioscler Thromb Vasc Biol 20:2539-2545. 
Kintscher U, Wakino S, Kim S, Fleck E, Hsueh WA, and Law RE (2001) Angiotensin II induces migration and Pyk2/paxillin phosphorylation of human monocytes. Hypertension 37:587-593.

Kippenberger S, Loitsch S, Guschel M, Müller J, Knies Y, Kaufmann R, and Bernd A (2005) Mechanical stretch stimulates protein kinase B/Akt phosphorylation in epidermal cells via angiotensin II type 1 receptor and epidermal growth factor receptor. J Biol Chem 280:3060-3067.

Kiya Y, Miura S, Fujino M, Imaizumi S, Karnik SS, and Saku K (2010) Clinical and pharmacotherapeutic relevance of the double-chain domain of the angiotensin II type 1 receptor blocker olmesartan. Clin Exp Hypertens 32:129-136.

Kluskens LD, Nelemans SA, Rink R, de Vries L, Meter-Arkema A, Wang Y, Walther T, Kuipers A, Moll GN, and Haas M (2009) Angiotensin-(1-7) with thioether bridge: an angiotensin-converting enzyme-resistant, potent angiotensin-(1-7) analog. $J$ Pharmacol Exp Ther 328:849-854.

Knowle D, Ahmed S, and Pulakat L (2000) Identification of an interaction between the angiotensin II receptor sub-type AT2 and the ErbB3 receptor, a member of the epidermal growth factor receptor family. Regul Pept 87:73-82.

Kohara Y, Kubo K, Imamiya E, Wada T, Inada Y, and Naka T (1996) Synthesis and angiotensin II receptor antagonistic activities of benzimidazole derivatives bearing acidic heterocycles as novel tetrazole bioisosteres. J Med Chem 39:5228-5235.

Kohout TA, Lin FS, Perry SJ, Conner DA, and Lefkowitz RJ (2001) beta-Arrestin 1 and 2 differentially regulate heptahelical receptor signaling and trafficking. Proc Natl Acad Sci USA 98:1601-1606.

Koike G, Horiuchi M, Yamada T, Szpirer C, Jacob HJ, and Dzau VJ (1994) Human type 2 angiotensin II receptor gene: cloned, mapped to the $\mathrm{X}$ chromosome, and its mRNA is expressed in the human lung. Biochem Biophys Res Commun 203: 1842-1850.

Kostenis E, Milligan G, Christopoulos A, Sanchez-Ferrer CF, Heringer-Walther S, Sexton PM, Gembardt F, Kellett E, Martini L, and Vanderheyden P, et al. (2005) G-protein-coupled receptor Mas is a physiological antagonist of the angiotensin II type 1 receptor. Circulation 111:1806-1813.

Kotani K, Fujiwara S, Tsuzaki K, Sano Y, Matsuoka Y, Hamada T, and Sakane N (2009) An association between angiotensin II type 2 receptor gene A/C3123 polymorphism and glycemic control marker in a general Japanese population. Mol Biol Rep 36:917-920.

Kotani K, Sakane N, Saiga K, Tsuzaki K, Sano Y, Mu H, and Kurozawa Y (2007) The angiotensin II type 2 receptor gene polymorphism and body mass index in healthy Japanese women. Ann Clin Biochem 44:83-85.

Kramár EA, Armstrong DL, Ikeda S, Wayner MJ, Harding JW, and Wright JW (2001) The effects of angiotensin IV analogs on long-term potentiation within the CA1 region of the hippocampus in vitro. Brain Res 897:114-121.

Kramár EA, Harding JW, and Wright JW (1997) Angiotensin II- and IV-induced changes in cerebral blood flow. Roles of AT1, AT2, and AT4 receptor subtypes. Regul Pept 68:131-138.

Kramár EA, Krishnan R, Harding JW, and Wright JW (1998) Role of nitric oxide in angiotensin IV-induced increases in cerebral blood flow. Regul Pept 74:185-192.

Kranenburg O and Moolenaar WH (2001) Ras-MAP kinase signaling by lysophosphatidic acid and other $\mathrm{G}$ protein-coupled receptor agonists. Oncogene 20 1540-1546.

Kranzhöfer R, Schmidt J, Pfeiffer CA, Hagl S, Libby P, and Kübler W (1999) Angiotensin induces inflammatory activation of human vascular smooth muscle cells. Arterioscler Thromb Vasc Biol 19:1623-1629.

Krebs LT, Kramár EA, Hanesworth JM, Sardinia MF, Ball AE, Wright JW, and Harding JW (1996) Characterization of the binding properties and physiological action of divalinal-angiotensin IV, a putative AT4 receptor antagonist. Regul Pept 67:123-130.

Kule CE, Karoor V, Day JN, Thomas WG, Baker KM, Dinh D, Acker KA, and Booz GW (2004) Agonist-dependent internalization of the angiotensin II type one receptor (AT1): role of C-terminus phosphorylation in recruitment of beta-arrestins. Regul Pept 120:141-148.

Kumar M, Grammas P, Giacomelli F, and Wiener J (1996) Selective expression of c-mas proto-oncogene in rat cerebral endothelial cells. Neuroreport 8:93-96.

Kumar R and Boim MA (2009) Diversity of pathways for intracellular angiotensin II synthesis. Curr Opin Nephrol Hypertens 18:33-39.

Kumar R, Singh VP, and Baker KM (2008) The intracellular renin-angiotensin system: implications in cardiovascular remodeling. Curr Opin Nephrol Hypertens 17: 168-173.

Kumar R, Singh VP, and Baker KM (2009) The intracellular renin-angiotensin system in the heart. Curr Hypertens Rep 11:104-110.

Kumar R, Yong QC, Thomas CM, and Baker KM (2012) Intracardiac intracellular angiotensin system in diabetes. Am J Physiol Regul Integr Comp Physiol 302 R510-R517.

Kumar V, Knowle D, Gavini N, and Pulakat L (2002) Identification of the region of AT2 receptor needed for inhibition of the AT1 receptor-mediated inositol 1,4,5triphosphate generation. FEBS Lett 532:379-386.

Kunz J, Krause D, Kremer M, and Dermietzel R (1994) The 140-kDa protein of bloodbrain barrier-associated pericytes is identical to aminopeptidase N. J Neurochem 62:2375-2386.

Kupfahl C, Pink D, Friedrich K, Zurbrügg HR, Neuss M, Warnecke C, Fielitz J, Graf K, Fleck E, and Regitz-Zagrosek V (2000) Angiotensin II directly increases transforming growth factor beta1 and osteopontin and indirectly affects collagen mRNA expression in the human heart. Cardiovasc Res 46:463-475.

Kurikawa N, Suga M, Kuroda S, Yamada K, and Ishikawa H (2003) An angiotensin II type 1 receptor antagonist, olmesartan medoxomil, improves experimental liver fibrosis by suppression of proliferation and collagen synthesis in activated hepatic stellate cells. Br J Pharmacol 139:1085-1094.

Kurisu S, Ozono R, Oshima T, Kambe M, Ishida T, Sugino H, Matsuura H, Chayama $\mathrm{K}$, Teranishi Y, and Iba O, et al. (2003) Cardiac angiotensin II type 2 receptor activates the kinin/NO system and inhibits fibrosis. Hypertension 41:99-107.
Kurosaka M, Suzuki T, Hosono K, Kamata Y, Fukamizu A, Kitasato H, Fujita Y, and Majima M (2009) Reduced angiogenesis and delay in wound healing in angiotensin II type 1a receptor-deficient mice. Biomed Pharmacother 63:627-634.

Kurtz TW and Pravenec M (2004) Antidiabetic mechanisms of angiotensinconverting enzyme inhibitors and angiotensin II receptor antagonists: beyond the renin-angiotensin system. J Hypertens 22:2253-2261.

Laflamme L, Gasparo M, Gallo JM, Payet MD, and Gallo-Payet N (1996) Angiotensin II induction of neurite outgrowth by AT2 receptors in NG108-15 cells. Effect counteracted by the AT1 receptors. J Biol Chem 271:22729-22735.

LaMarca B, Wallace K, and Granger J (2011) Role of angiotensin II type I receptor agonistic autoantibodies (AT1-AA) in preeclampsia. Curr Opin Pharmacol 11 $175-179$

Lanckmans K, Sarre S, Smolders I, and Michotte Y (2007a) Use of a structural analogue versus a stable isotope labeled internal standard for the quantification of angiotensin IV in rat brain dialysates using nano-liquid chromatography/tandem mass spectrometry. Rapid Commun Mass Spectrom 21:1187-1195.

Lanckmans K, Stragier B, Sarre S, Smolders I, and Michotte Y (2007b) Nano-LC-MS/ MS for the monitoring of angiotensin IV in rat brain microdialysates: limitations and possibilities. $J$ Sep Sci 30:2217-2224.

Langeveld BE, Henning RH, de Smet BJ, Zijlstra F, Driessen A, Tijsma E, van Gilst WH, and Roks A (2008) Rescue of arterial function by angiotensin-(1-7): towards improvement of endothelial function by drug-eluting stents. Neth Heart $J$ 16:291-292.

Larance M, Ramm G, Stöckli J, van Dam EM, Winata S, Wasinger V, Simpson F, Graham M, Junutula JR, and Guilhaus M, et al. (2005) Characterization of the role of the Rab GTPase-activating protein AS160 in insulin-regulated GLUT4 trafficking. J Biol Chem 280:37803-37813.

Lau ST and Leung PS (2011) Role of the RAS in pancreatic cancer. Curr Cancer Drug Targets 11:412-420.

Lautner RQ, Villela DC, Fraga-Silva RA, Silva N, Verano-Braga T, Costa-Fraga F, Jankowski J, Jankowski V, Sousa F, and Alzamora A, et al. (2013) Discovery and characterization of alamandine: a novel component of the renin-angiotensin system. Circ Res 112:1104-1111.

Lautrette A, Li S, Alili R, Sunnarborg SW, Burtin M, Lee DC, Friedlander G, and Terzi F (2005) Angiotensin II and EGF receptor cross-talk in chronic kidney diseases: a new therapeutic approach. Nat Med 11:867-874.

Lavoie JL and Sigmund CD (2003) Minireview: overview of the renin-angiotensin system-an endocrine and paracrine system. Endocrinology 144:2179-2183.

Lax CJ, Domenighetti AA, Pavia JM, Di Nicolantonio R, Curl CL, Morris MJ, and Delbridge LM (2004) Transitory reduction in angiotensin AT2 receptor expression levels in postinfarct remodelling in rat myocardium. Clin Exp Pharmacol Physiol 31:512-517.

Lazartigues E, Dunlay SM, Loihl AK, Sinnayah P, Lang JA, Espelund JJ, Sigmund CD, and Davisson RL (2002) Brain-selective overexpression of angiotensin (AT1) receptors causes enhanced cardiovascular sensitivity in transgenic mice. Circ Res 90:617-624.

Lazartigues E, Lawrence AJ, Lamb FS, and Davisson RL (2004) Renovascular hypertension in mice with brain-selective overexpression of AT1a receptors is buffered by increased nitric oxide production in the periphery. Circ Res 95:523-531.

Lazartigues E, Sinnayah P, Augoyard G, Gharib C, Johnson AK, and Davisson RL (2008) Enhanced water and salt intake in transgenic mice with brain-restricted overexpression of angiotensin (AT1) receptors. Am J Physiol Regul Integr Comp Physiol 295:R1539-R1545.

Le MT, De Backer JP, Hunyady L, Vanderheyden PM, and Vauquelin G (2005) Ligand binding and functional properties of human angiotensin AT1 receptors in transiently and stably expressed CHO-K1 cells. Eur J Pharmacol 513:35-45.

Le MT, Vanderheyden PM, Szaszák M, Hunyady L, and Vauquelin G (2002) Angiotensin IV is a potent agonist for constitutive active human AT1 receptors. Distinct roles of the $\mathrm{N}$-and C-terminal residues of angiotensin II during AT1 receptor activation. J Biol Chem 277:23107-23110.

Leal MC, Pinheiro SV, Ferreira AJ, Santos RA, Bordoni LS, Alenina N, Bader M, and Franca LR (2009) The role of angiotensin-(1-7) receptor Mas in spermatogenesis in mice and rats. J Anat 214:736-743.

Leduc I and Meloche S (1995) Angiotensin II stimulates tyrosine phosphorylation of the focal adhesion-associated protein paxillin in aortic smooth muscle cells. J Biol Chem 270:4401-4404.

Lee HW (2014) Angiotensin receptor blocker add-on therapy in portal hypertension to use angiotensin receptor blocker or not to use, that is the question. Clin $\mathrm{Mol}$ Hepatol 20:345-346.

Lee J, Albiston AL, Allen AM, Mendelsohn FA, Ping SE, Barrett GL, Murphy M, Morris MJ, McDowall SG, and Chai SY (2004) Effect of I.C.V. injection of AT4 receptor ligands, NLE1-angiotensin IV and LVV-hemorphin 7, on spatial learning in rats. Neuroscience 124:341-349.

Lee J, Chai SY, Mendelsohn FA, Morris MJ, and Allen AM (2001) Potentiation of cholinergic transmission in the rat hippocampus by angiotensin IV and LVVhemorphin-7. Neuropharmacology 40:618-623.

Lee J, Mustafa T, McDowall SG, Mendelsohn FA, Brennan M, Lew RA, Albiston AL, and Chai SY (2003) Structure-activity study of LVV-hemorphin-7: angiotensin AT4 receptor ligand and inhibitor of insulin-regulated aminopeptidase. J Pharmacol Exp Ther 305:205-211.

Lee MH, El-Shewy HM, Luttrell DK, and Luttrell LM (2008) Role of beta-arrestinmediated desensitization and signaling in the control of angiotensin AT1a receptor-stimulated transcription. J Biol Chem 283:2088-2097.

Lefkowitz RJ (1998) G protein-coupled receptors. III. New roles for receptor kinases and beta-arrestins in receptor signaling and desensitization. J Biol Chem 273: $18677-18680$.

Lefkowitz RJ and Shenoy SK (2005) Transduction of receptor signals by betaarrestins. Science 308:512-517.

Lehtonen JY, Daviet L, Nahmias C, Horiuchi M, and Dzau VJ (1999) Analysis of functional domains of angiotensin II type 2 receptor involved in apoptosis. $\mathrm{Mol}$ Endocrinol 13:1051-1060. 
Lemarié CA and Schiffrin EL (2010) The angiotensin II type 2 receptor in cardiovascular disease. $J$ Renin Angiotensin Aldosterone Syst 11:19-31.

Lembo PM, Grazzini E, Groblewski T, O’Donnell D, Roy MO, Zhang J, Hoffert C, Cao J, Schmidt R, and Pelletier M, et al. (2002) Proenkephalin A gene products activate a new family of sensory neuron-specific GPCRs. Nat Neurosci 5:201-209.

Lenkei Z, Palkovits M, Corvol P, and Llorens-Cortès C (1997) Expression of angiotensin type-1 (AT1) and type-2 (AT2) receptor mRNAs in the adult rat brain: a functional neuroanatomical review. Front Neuroendocrinol 18:383-439.

Leung KH, Chang RS, Lotti VJ, Roscoe WA, Smith RD, Timmermans PB, and Chiu AT (1992a) AT1 receptors mediate the release of prostaglandins in porcine smooth muscle cells and rat astrocytes. Am J Hypertens 5:648-656.

Leung KH, Roscoe WA, Smith RD, Timmermans PB, and Chiu AT (1992b) Characterization of biochemical responses of angiotensin II (AT2) binding sites in the rat pheochromocytoma PC12W cells. Eur J Pharmacol 227:63-70.

Levy BI, Benessiano J, Henrion D, Caputo L, Heymes C, Duriez M, Poitevin P, and Samuel JL (1996) Chronic blockade of AT2-subtype receptors prevents the effect of angiotensin II on the rat vascular structure. J Clin Invest 98:418-425.

Lew RA, Mustafa T, Ye S, McDowall SG, Chai SY, and Albiston AL (2003) Angiotensin AT4 ligands are potent, competitive inhibitors of insulin regulated aminopeptidase (IRAP). $J$ Neurochem 86:344-350.

Li A, Rockne KJ, Sturchio N, Song W, Ford JC, and Wei H (2009a) PCBs in sediments of the Great Lakes-distribution and trends, homolog and chlorine patterns, and in situ degradation. Environ Pollut 157:141-147.

Li D and Mehta JL (2000) Upregulation of endothelial receptor for oxidized LDL (LOX-1) by oxidized LDL and implications in apoptosis of human coronary artery endothelial cells: evidence from use of antisense LOX-1 mRNA and chemical inhibitors. Arterioscler Thromb Vasc Biol 20:1116-1122.

Li D, Saldeen T, Romeo F, and Mehta JL (2000) Oxidized LDL upregulates angiotensin II type 1 receptor expression in cultured human coronary artery endothelial cells: the potential role of transcription factor NF-kappaB. Circulation 102 1970-1976.

Li H, Qi Y, Li C, Braseth LN, Gao Y, Shabashvili AE, Katovich MJ, and Sumners C (2009b) Angiotensin type 2 receptor-mediated apoptosis of human prostate cancer cells. Mol Cancer Ther 8:3255-3265.

Li H, Weatherford ET, Davis DR, Keen HL, Grobe JL, Daugherty A, Cassis LA, Allen AM, and Sigmund CD (2011) Renal proximal tubule angiotensin AT1A receptors regulate blood pressure. Am J Physiol Regul Integr Comp Physiol 301: R1067-R1077.

Li J, Culman J, Hortnagl H, Zhao Y, Gerova N, Timm M, Blume A, Zimmermann M, Seidel K, and Dirnagl U, et al. (2005) Angiotensin AT2 receptor protects against cerebral ischemia-induced neuronal injury. FASEB $J$ 19:617-619.

Li J, Zhao X, Li X, Lerea KM, and Olson SC (2007a) Angiotensin II type 2 receptordependent increases in nitric oxide synthase expression in the pulmonary endothelium is mediated via a $\mathrm{G}$ alpha i3/Ras/Raf/MAPK pathway. Am $\mathrm{J}$ Physiol Cell Physiol 292:C2185-C2196.

Li JM, Mogi M, Tsukuda K, Tomochika H, Iwanami J, Min LJ, Nahmias C, Iwai M, and Horiuchi M (2007b) Angiotensin II-induced neural differentiation via angiotensin II type 2 (AT2) receptor-MMS2 cascade involving interaction between AT2 receptor-interacting protein and Src homology 2 domain-containing proteintyrosine phosphatase 1. Mol Endocrinol 21:499-511.

Li P, Sun HJ, Cui BP, Zhou YB, and Han Y (2013) Angiotensin-(1-7) in the rostral ventrolateral medulla modulates enhanced cardiac sympathetic afferent reflex and sympathetic activation in renovascular hypertensive rats. Hypertension 61 : 820-827.

Li W, Peng H, Mehaffey EP, Kimball CD, Grobe JL, van Gool JM, Sullivan MN, Earley S, Danser AH, and Ichihara A, et al. (2014) Neuron-specific (pro)renin receptor knockout prevents the development of salt-sensitive hypertension. Hypertension 63:316-323.

Li XC, Shao Y, and Zhuo JL (2009c) AT1a receptor knockout in mice impairs urine concentration by reducing basal vasopressin levels and its receptor signaling proteins in the inner medulla. Kidney Int 76:169-177.

Li YD, Block ER, and Patel JM (2002) Activation of multiple signaling modules is critical in angiotensin IV-induced lung endothelial cell proliferation. Am J Physiol Lung Cell Mol Physiol 283:L707-L716.

Liao DF, Duff JL, Daum G, Pelech SL, and Berk BC (1996) Angiotensin II stimulates MAP kinase kinase kinase activity in vascular smooth muscle cells, Role of Raf. Circ Res 79:1007-1014.

Liao DF, Monia B, Dean N, and Berk BC (1997) Protein kinase C-zeta mediates angiotensin II activation of ERK1/2 in vascular smooth muscle cells. J Biol Chem 272:6146-6150.

Liao YH, Wei YM, Wang M, Wang ZH, Yuan HT, and Cheng LX (2002) Autoantibodies against AT1-receptor and alpha1-adrenergic receptor in patients with hypertension. Hypertens Res 25:641-646.

Lijnen P and Petrov V (1999) Renin-angiotensin system, hypertrophy and gene expression in cardiac myocytes. $J \mathrm{Mol}$ Cell Cardiol 31:949-970.

Lim H and Zhu YZ (2006) Role of transforming growth factor-beta in the progression of heart failure. Cell Mol Life Sci 63:2584-2596.

Linseman DA, Benjamin CW, and Jones DA (1995) Convergence of angiotensin II and platelet-derived growth factor receptor signaling cascades in vascular smooth muscle cells. J Biol Chem 270:12563-12568.

Lips DJ, deWindt LJ, van Kraaij DJ, and Doevendans PA (2003) Molecular determinants of myocardial hypertrophy and failure: alternative pathways for beneficial and maladaptive hypertrophy. Eur Heart $J$ 24:883-896.

Liu G, Hitomi H, Hosomi N, Lei B, Pelisch N, Nakano D, Kiyomoto H, Ma H, and Nishiyama A (2010) Mechanical stretch potentiates angiotensin II-induced proliferation in spontaneously hypertensive rat vascular smooth muscle cells. Hypertens Res 33:1250-1257.

Liu GC, Oudit GY, Fang F, Zhou J, and Scholey JW (2012) Angiotensin-(1-7)-induced activation of ERK1/2 is cAMP/protein kinase A-dependent in glomerular mesangial cells. Am J Physiol Renal Physiol 302:F784-F790.
Liu HQ, Wei XB, Sun R, Cai YW, Lou HY, Wang JW, Chen AF, and Zhang XM (2006) Angiotensin II stimulates intercellular adhesion molecule-1 via an AT1 receptor/ nuclear factor-kappaB pathway in brain microvascular endothelial cells. Life Sci 78:1293-1298.

Liu Q, Tang Z, Surdenikova L, Kim S, Patel KN, Kim A, Ru F, Guan Y, Weng HJ, and Geng Y, et al. (2009) Sensory neuron-specific GPCR Mrgprs are itch receptors mediating chloroquine-induced pruritus. Cell 139:1353-1365.

Liu YH, Yang XP, Sharov VG, Nass O, Sabbah HN, Peterson E, and Carretero OA (1997) Effects of angiotensin-converting enzyme inhibitors and angiotensin II type 1 receptor antagonists in rats with heart failure. Role of kinins and angiotensin II type 2 receptors. J Clin Invest 99:1926-1935.

Lo M, Liu KL, Lantelme P, and Sassard J (1995) Subtype 2 of angiotensin II receptors controls pressure-natriuresis in rats. J Clin Invest 95:1394-1397.

Lochard N, Thibault G, Silversides DW, Touyz RM, and Reudelhuber TL (2004) Chronic production of angiotensin IV in the brain leads to hypertension that is reversible with an angiotensin II AT1 receptor antagonist. Circ Res 94:1451-1457.

Lodwick D (2001) Receptor double-trouble in preeclampsia. Nat Med 7:999-1000.

Lokuta AJ, Cooper C, Gaa ST, Wang HE, and Rogers TB (1994) Angiotensin II stimulates the release of phospholipid-derived second messengers through multiple receptor subtypes in heart cells. J Biol Chem 269:4832-4838.

Lombardi D, Gordon KL, Polinsky P, Suga S, Schwartz SM, and Johnson RJ (1999) Salt-sensitive hypertension develops after short-term exposure to Angiotensin II. Hypertension 33:1013-1019.

Lopez-Ilasaca M, Crespo P, Pellici PG, Gutkind JS, and Wetzker R (1997) Linkage of $\mathrm{G}$ protein-coupled receptors to the MAPK signaling pathway through PI 3-kinase gamma. Science 275:394-397.

Lopez-Ilasaca M, Liu X, Tamura K, and Dzau VJ (2003) The angiotensin II type I receptor-associated protein, ATRAP, is a transmembrane protein and a modulator of angiotensin II signaling. Mol Biol Cell 14:5038-5050.

Lopez Verrilli MA, Rodriguez Fermepín M, Longo Carbajosa N, Landa S, Cerrato BD, García S, Fernandez BE, and Gironacci MM (2012) Angiotensin-(1-7) through Mas receptor up-regulates neuronal norepinephrine transporter via Akt and Erk1/2dependent pathways. J Neurochem 120:46-55.

Loyens E, Schallier A, Chai SY, De Bundel D, Vanderheyden P, Michotte Y, and Smolders I (2011) Deletion of insulin-regulated aminopeptidase in mice decreases susceptibility to pentylenetetrazol-induced generalized seizures. Seizure 20:602-605.

Luchtefeld M, Drexler H, and Schieffer B (2001) Role of G beta-subunit in angiotensin II-type 1 receptor signaling. Biochem Biophys Res Commun 280:756-760.

Luft FC (2004) Present status of genetic mechanisms in hypertension. Med Clin North Am 88:1-18.

Lukaszuk A, Demaegdt H, Feytens D, Vanderheyden P, Vauquelin G, and Tourwé D (2009) The replacement of His(4) in angiotensin IV by conformationally constrained residues provides highly potent and selective analogues. J Med Chem $\mathbf{5 2}$ : $5612-5618$

Lukaszuk A, Demaegdt H, Szemenyei E, Tóth G, Tymecka D, Misicka A, Karoyan P, Vanderheyden P, Vauquelin G, and Tourwé D (2008) Beta-homo-amino acid scan of angiotensin IV. J Med Chem 51:2291-2296.

Lukaszuk A, Demaegdt H, Van den Eynde I, Vanderheyden P, Vauquelin G, and Tourwe D (2011) Conformational constraints in angiotensin IV to probe the role of Tyr(2), Pro(5) and Phe(6). J Pept Sci 17:545-553.

Luttrell LM, Ferguson SS, Daaka Y, Miller WE, Maudsley S, Della Rocca GJ, Lin F, Kawakatsu H, Owada K, and Luttrell DK, et al. (1999) Beta-arrestin-dependent formation of beta 2 adrenergic receptor-Src protein kinase complexes. Science $\mathbf{2 8 3}$ : $655-661$.

Luttrell LM, Roudabush FL, Choy EW, Miller WE, Field ME, Pierce KL, and Lefkowitz RJ (2001) Activation and targeting of extracellular signal-regulated kinases by beta-arrestin scaffolds. Proc Natl Acad Sci USA 98:2449-2454.

Lyngsø C, Erikstrup N, and Hansen JL (2009) Functional interactions between 7TM receptors in the renin-angiotensin system-dimerization or crosstalk? Mol Cell Endocrinol 302:203-212.

Macari D, Whitebread S, Cumin F, De Gasparo M, and Levens N (1994) Renal action of the angiotensin AT2 receptor ligands CGP 42112 and PD 123319 after blockade of the renin-angiotensin system. Eur J Pharmacol 259:27-36.

Madamanchi NR, Li S, Patterson C, and Runge MS (2001) Reactive oxygen species regulate heat-shock protein 70 via the JAK/STAT pathway. Arterioscler Thromb Vasc Biol 21:321-326.

Magaldi AJ, Cesar KR, de Araújo M, Simões e Silva AC, and Santos RA (2003) Angiotensin-(1-7) stimulates water transport in rat inner medullary collecting duct: evidence for involvement of vasopressin V2 receptors. Pflugers Arch 447: $223-230$.

Maillard MP, Würzner G, Nussberger J, Centeno C, Burnier M, and Brunner HR (2002) Comparative angiotensin II receptor blockade in healthy volunteers: the importance of dosing. Clin Pharmacol Ther 71:68-76.

Malik RA (2000) Can diabetic neuropathy be prevented by angiotensin-converting enzyme inhibitors? Ann Med 32:1-5.

Malik RA, Williamson S, Abbott C, Carrington AL, Iqbal J, Schady W, and Boulton AJ (1998) Effect of angiotensin-converting-enzyme (ACE) inhibitor trandolapril on human diabetic neuropathy: randomised double-blind controlled trial. Lancet $\mathbf{3 5 2}$ : 1978-1981.

Many A, Hubel CA, Fisher SJ, Roberts JM, and Zhou Y (2000) Invasive cytotrophoblasts manifest evidence of oxidative stress in preeclampsia. Am J Pathol 156:321-331.

Marie J, Maigret B, Joseph MP, Larguier R, Nouet S, Lombard C, and Bonnafous JC (1994) Tyr292 in the seventh transmembrane domain of the AT1A angiotensin II receptor is essential for its coupling to phospholipase C. J Biol Chem 269: 20815-20818.

Mario EG, Santos SH, Ferreira AV, Bader M, Santos RA, and Botion LM (2012) Angiotensin-(1-7) Mas-receptor deficiency decreases peroxisome proliferatoractivated receptor gamma expression in adipocytes. Peptides 33:174-177. 
Marrero MB, Schieffer B, Paxton WG, Heerdt L, Berk BC, Delafontaine P, and Bernstein KE (1995a) Direct stimulation of Jak/STAT pathway by the angiotensin II AT1 receptor. Nature 375:247-250.

Marrero MB, Schieffer B, Paxton WG, Schieffer E, and Bernstein KE (1995b) Electroporation of pp60c-src antibodies inhibits the angiotensin II activation of phospholipase C-gamma 1 in rat aortic smooth muscle cells. J Biol Chem 270:15734-15738.

Marrero MB, Venema VJ, Ju H, Eaton DC, and Venema RC (1998) Regulation of angiotensin II-induced JAK2 tyrosine phosphorylation: roles of SHP-1 and SHP-2. Am J Physiol 275:C1216-C1223.

Martin KA, Grant SG, and Hockfield S (1992) The mas proto-oncogene is developmentally regulated in the rat central nervous system. Brain Res Dev Brain Res 68:75-82.

Martin KA and Hockfield S (1993) Expression of the mas proto-oncogene in the rat hippocampal formation is regulated by neuronal activity. Brain Res Mol Brain Res 19:303-309.

Martin SS, Boucard AA, Clément M, Escher E, Leduc R, and Guillemette G (2004) Analysis of the third transmembrane domain of the human type 1 angiotensin II receptor by cysteine scanning mutagenesis. J Biol Chem 279:51415-51423.

Martin SS, Holleran BJ, Escher E, Guillemette G, and Leduc R (2007) Activation of the angiotensin II type 1 receptor leads to movement of the sixth transmembrane domain: analysis by the substituted cysteine accessibility method. Mol Pharmacol 72:182-190.

Marui N, Offermann MK, Swerlick R, Kunsch C, Rosen CA, Ahmad M, Alexander RW, and Medford RM (1993) Vascular cell adhesion molecule-1 (VCAM-1) gene transcription and expression are regulated through an antioxidant-sensitive mechanism in human vascular endothelial cells. J Clin Invest 92:1866-1874.

Masaki H, Kurihara T, Yamaki A, Inomata N, Nozawa Y, Mori Y, Murasawa S, Kizima K, Maruyama K, and Horiuchi M, et al. (1998) Cardiac-specific overexpression of angiotensin II AT2 receptor causes attenuated response to AT1 receptor-mediated pressor and chronotropic effects. J Clin Invest 101:527-535.

Matsubara H, Shibasaki Y, Okigaki M, Mori Y, Masaki H, Kosaki A, Tsutsumi Y, Uchiyama Y, Fujiyama S, and Nose A, et al. (2001) Effect of angiotensin II type 2 receptor on tyrosine kinase Pyk2 and c-Jun NH2-terminal kinase via SHP-1 tyrosine phosphatase activity: evidence from vascular-targeted transgenic mice of AT2 receptor. Biochem Biophys Res Commun 282:1085-1091.

Matsumoto H, Nagasaka T, Hattori A, Rogi T, Tsuruoka N, Mizutani S, and Tsujimoto M (2001) Expression of placental leucine aminopeptidase/ oxytocinase in neuronal cells and its action on neuronal peptides. Eur J Biochem 268:3259-3266.

Matsumoto T, Ozono R, Oshima T, Matsuura H, Sueda T, Kajiyama G, and Kambe M (2000) Type 2 angiotensin II receptor is downregulated in cardiomyocytes of patients with heart failure. Cardiovasc Res 46:73-81.

McAllister-Lucas LM, Ruland J, Siu K, Jin X, Gu S, Kim DS, Kuffa P, Kohrt D, Mak TW, and Nuñez G, et al. (2007) CARMA3/Bcl10/MALT1-dependent NF-kappaB activation mediates angiotensin II-responsive inflammatory signaling in nonimmune cells. Proc Natl Acad Sci USA 104:139-144.

McGillis JP, Sudduth-Klinger J, Harrowe G, Mitsuhashi M, and Payan DG (1989) Transient expression of the angiotensin II receptor: a rapid and functional analysis of a calcium-mobilizing seven-transmembrane domain receptor in COS-7 cells. Biochem Biophys Res Commun 165:935-941.

Mecca AP, Regenhardt RW, O'Connor TE, Joseph JP, Raizada MK, Katovich MJ, and Sumners C (2011) Cerebroprotection by angiotensin-(1-7) in endothelin-1induced ischaemic stroke. Exp Physiol 96:1084-1096.

Mederos y Schnitzler M, Storch U, and Gudermann T (2011) AT1 receptors as mechanosensors. Curr Opin Pharmacol 11:112-116.

Meffert S, Stoll M, Steckelings UM, Bottari SP, and Unger T (1996) The angiotensin II AT2 receptor inhibits proliferation and promotes differentiation in PC12W cells. Mol Cell Endocrinol 122:59-67.

Mehta PK and Griendling KK (2007) Angiotensin II cell signaling: physiological and pathological effects in the cardiovascular system. Am J Physiol Cell Physiol 292 C82-C97.

Mercure C, Yogi A, Callera GE, Aranha AB, Bader M, Ferreira AJ, Santos RA, Walther T, Touyz RM, and Reudelhuber TL (2008) Angiotensin(1-7) blunts hypertensive cardiac remodeling by a direct effect on the heart. Circ Res 103 $1319-1326$

Mertens B, Vanderheyden P, Michotte Y, and Sarre S (2010) Direct angiotensin II type 2 receptor stimulation decreases dopamine synthesis in the rat striatum. Neuropharmacology 58:1038-1044

Metzger R, Bader M, Ludwig T, Berberich C, Bunnemann B, and Ganten D (1995) Expression of the mouse and rat mas proto-oncogene in the brain and peripheral tissues. FEBS Lett 357:27-32.

Meyer P, Papadimitriou A, and Worcel M (1974) Specific supersensitivity of smooth muscle to angiotensin II after nephrectomy. Br J Pharmacol 51:435-439.

Michel MC, Foster C, Brunner HR, and Liu L (2013) A systematic comparison of the properties of clinically used angiotensin II type 1 receptor antagonists. Pharmacol Rev 65:809-848.

Mifune M, Ohtsu H, Suzuki H, Nakashima H, Brailoiu E, Dun NJ, Frank GD, Inagami T, Higashiyama $S$, and Thomas WG, et al. (2005) $G$ protein coupling and second messenger generation are indispensable for metalloprotease-dependent, heparin-binding epidermal growth factor shedding through angiotensin II type-1 receptor. J Biol Chem 280:26592-26599.

Mifune M, Sasamura H, Shimizu-Hirota R, Miyazaki H, and Saruta T (2000) Angiotensin II type 2 receptors stimulate collagen synthesis in cultured vascular smooth muscle cells. Hypertension 36:845-850.

Miller-Wing AV, Hanesworth JM, Sardinia MF, Hall KL, Wright JW, Speth RC, Grove KL, and Harding JW (1993) Central angiotensin IV binding sites: distribution and specificity in guinea pig brain. J Pharmacol Exp Ther 266:1718-1726.

Miura S, Feng YH, Husain A, and Karnik SS (1999) Role of aromaticity of agonist switches of angiotensin II in the activation of the AT1 receptor. J Biol Chem 274: $7103-7110$
Miura S, Fujino M, Hanzawa H, Kiya Y, Imaizumi S, Matsuo Y, Tomita S, Uehara Y, Karnik SS, and Yanagisawa H, et al. (2006) Molecular mechanism underlying inverse agonist of angiotensin II type 1 receptor. J Biol Chem 281:19288-19295.

Miura S and Karnik SS (1999) Angiotensin II type 1 and type 2 receptors bind angiotensin II through different types of epitope recognition. J Hypertens $\mathbf{1 7}$ 397-404.

Miura S and Karnik SS (2000) Ligand-independent signals from angiotensin II type 2 receptor induce apoptosis. EMBO J 19:4026-4035.

Miura S and Karnik SS (2002) Constitutive activation of angiotensin II type 1 receptor alters the orientation of transmembrane Helix-2. J Biol Chem 277: 24299-24305.

Miura S, Karnik SS, and Saku K (2005) Constitutively active homo-oligomeric angiotensin II type 2 receptor induces cell signaling independent of receptor conformation and ligand stimulation. J Biol Chem 280:18237-18244.

Miura S, Karnik SS, and Saku K (2011) Review: angiotensin II type 1 receptor blockers: class effects versus molecular effects. J Renin Angiotensin Aldosterone Syst 12:1-7.

Miura S, Kiya Y, Hanzawa H, Nakao N, Fujino M, Imaizumi S, Matsuo Y, Yanagisawa H, Koike H, and Komuro I, et al. (2012) Small molecules with similar structures exhibit agonist, neutral antagonist or inverse agonist activity toward angiotensin II type 1 receptor. PLoS One 7:e37974.

Miura S, Matsuo Y, Kiya Y, Karnik SS, and Saku K (2010) Molecular mechanisms of the antagonistic action between AT1 and AT2 receptors. Biochem Biophys Res Commun 391:85-90.

Miura S, Okabe A, Matsuo Y, Karnik SS, and Saku K (2013) Unique binding behavior of the recently approved angiotensin II receptor blocker azilsartan compared with that of candesartan. Hypertens Res 36:134-139.

Miura S, Saku K, and Karnik SS (2003a) Molecular analysis of the structure and function of the angiotensin II type 1 receptor. Hypertens Res 26:937-943.

Miura S, Zhang J, Boros J, and Karnik SS (2003b) TM2-TM7 interaction in coupling movement of transmembrane helices to activation of the angiotensin II type-1 receptor. J Biol Chem 278:3720-3725.

Miura S, Zhang J, and Karnik SS (2000) Angiotensin II type 1 receptor-function affected by mutations in cytoplasmic loop CD. FEBS Lett 470:331-335.

Miura S, Zhang J, Matsuo Y, Saku K, and Karnik SS (2004) Activation of extracellular signal-activated kinase by angiotensin II-induced Gq-independent epidermal growth factor receptor transactivation. Hypertens Res 27:765-770.

Miyaki K, Hara A, Araki J, Zhang L, Song Y, Kimura T, Omae K, and Muramatsu M (2006) C3123A polymorphism of the angiotensin II type 2 receptor gene and salt sensitivity in healthy Japanese men. J Hum Hypertens 20:467-469.

Mizuno M, Sada T, Ikeda M, Fukuda N, Miyamoto M, Yanagisawa H, and Koike H (1995) Pharmacology of CS-866, a novel nonpeptide angiotensin II receptor antagonist. Eur J Pharmacol 285:181-188.

Moeller I, Allen AM, Chai SY, Zhuo J, and Mendelsohn FA (1998) Bioactive angiotensin peptides. J Hum Hypertens 12:289-293.

Moeller I, Chai SY, Oldfield BJ, McKinley MJ, Casley D, and Mendelsohn FA (1995) Localization of angiotensin IV binding sites to motor and sensory neurons in the sheep spinal cord and hindbrain. Brain Res 701:301-306.

Moeller I, Lew RA, Mendelsohn FA, Smith AI, Brennan ME, Tetaz TJ, and Chai SY (1997) The globin fragment LVV-hemorphin-7 is an endogenous ligand for the AT4 receptor in the brain. $J$ Neurochem 68:2530-2537.

Moeller I, Paxinos G, Mendelsohn FA, Aldred GP, Casley D, and Chai SY (1996) Distribution of AT4 receptors in the Macaca fascicularis brain. Brain Res 712: 307-324.

Mogi M, Iwai M, and Horiuchi M (2009) New insights into the regulation of angiotensin receptors. Curr Opin Nephrol Hypertens 18:138-143.

Mondorf UF, Geiger H, Herrero M, Zeuzem S, and Piiper A (2000) Involvement of the platelet-derived growth factor receptor in angiotensin II-induced activation of extracellular regulated kinases 1 and 2 in human mesangial cells. FEBS Lett $\mathbf{4 7 2}$ 129-132.

Monnot C, Bihoreau C, Conchon S, Curnow KM, Corvol P, and Clauser E (1996) Polar residues in the transmembrane domains of the type 1 angiotensin II receptor are required for binding and coupling. Reconstitution of the binding site by coexpression of two deficient mutants. J Biol Chem 271:1507-1513.

Monnot C, Weber V, Stinnakre J, Bihoreau C, Teutsch B, Corvol P, and Clauser E (1991) Cloning and functional characterization of a novel mas-related gene, modulating intracellular angiotensin II actions. Mol Endocrinol 5:1477-1487.

Moon JY, Tanimoto M, Gohda T, Hagiwara S, Yamazaki T, Ohara I, Murakoshi M, Aoki T, Ishikawa Y, and Lee SH, et al. (2011) Attenuating effect of angiotensin(1-7) on angiotensin II-mediated $\mathrm{NAD}(\mathrm{P}) \mathrm{H}$ oxidase activation in type 2 diabetic nephropathy of KK-A(y)/Ta mice. Am J Physiol Renal Physiol 300:F1271-F1282.

Moore AF, Heiderstadt NT, Huang E, Howell NL, Wang ZQ, Siragy HM, and Carey RM (2001) Selective inhibition of the renal angiotensin type 2 receptor increase blood pressure in conscious rats. Hypertension 37:1285-1291.

Moreno M, Gonzalo T, Kok RJ, Sancho-Bru P, van Beuge M, Swart J, Prakash J, Temming K, Fondevila C, and Beljaars L, et al. (2010) Reduction of advanced liver fibrosis by short-term targeted delivery of an angiotensin receptor blocker to hepatic stellate cells in rats. Hepatology 51:942-952.

Mori S, Tanaka M, Nanba D, Nishiwaki E, Ishiguro $H$, Higashiyama $S$, and Matsuura N (2003) PACSIN3 binds ADAM12/meltrin alpha and up-regulates ectodomain shedding of heparin-binding epidermal growth factor-like growth factor. J Biol Chem 278:46029-46034.

Moriguchi Y, Matsubara H, Mori Y, Murasawa S, Masaki H, Maruyama K, Tsutsumi Y, Shibasaki Y, Tanaka Y, and Nakajima T, et al. (1999) Angiotensin II-induced transactivation of epidermal growth factor receptor regulates fibronectin and transforming growth factor-beta synthesis via transcriptional and posttranscriptional mechanisms. Circ Res 84:1073-1084.

Motley ED, Eguchi K, Gardner C, Hicks AL, Reynolds CM, Frank GD, Mifune M, Ohba M, and Eguchi S (2003) Insulin-induced Akt activation is inhibited by 
angiotensin II in the vasculature through protein kinase C-alpha. Hypertension 41 : $775-780$.

Mottl AK, Shoham DA, and North KE (2008) Angiotensin II type 1 receptor polymorphisms and susceptibility to hypertension: a HuGE review. Genet Med 10: 560-574.

Mountford SJ, Albiston AL, Charman WN, Ng L, Holien JK, Parker MW, Nicolazzo JA, Thompson PE, and Chai SY (2014) Synthesis, structure-activity relationships and brain uptake of a novel series of benzopyran inhibitors of insulin-regulated aminopeptidase. $J$ Med Chem 57:1368-1377.

Mukoyama M, Horiuchi M, Nakajima M, Pratt RE, and Dzau VJ (1995) Characterization of a rat type 2 angiotensin II receptor stably expressed in 293 cells. Mol Cell Endocrinol 112:61-68.

Mukoyama M, Nakajima M, Horiuchi M, Sasamura H, Pratt RE, and Dzau VJ (1993) Expression cloning of type 2 angiotensin II receptor reveals a unique class of seventransmembrane receptors. $J$ Biol Chem 268:24539-24542.

Müller P, Cohen T, de Gasparo M, Sioufi A, Racine-Poon A, and Howald H (1994) Angiotensin II receptor blockade with single doses of valsartan in healthy, normotensive subjects. Eur J Clin Pharmacol 47:231-245.

Munafo A, Christen Y, Nussberger J, Shum LY, Borland RM, Lee RJ, Waeber B, Biollaz J, and Brunner HR (1992) Drug concentration response relationships in normal volunteers after oral administration of losartan, an angiotensin II receptor antagonist. Clin Pharmacol Ther 51:513-521.

Munzenmaier DH and Greene AS (1996) Opposing actions of angiotensin II on microvascular growth and arterial blood pressure. Hypertension 27:760-765.

Murasawa S, Mori Y, Nozawa Y, Gotoh N, Shibuya M, Masaki H, Maruyama K, Tsutsumi Y, Moriguchi Y, and Shibazaki Y, et al. (1998) Angiotensin II type 1 receptor-induced extracellular signal-regulated protein kinase activation is mediated by $\mathrm{Ca} 2+/$ calmodulin-dependent transactivation of epidermal growth factor receptor. Circ Res 82:1338-1348.

Murphy TJ, Alexander RW, Griendling KK, Runge MS, and Bernstein KE (1991) Isolation of a cDNA encoding the vascular type-1 angiotensin II receptor. Nature 351:233-236.

Naffah-Mazzacoratti MG, Gouveia TL, Simões PS, and Perosa SR (2014) What have we learned about the kallikrein-kinin and renin-angiotensin systems in neurological disorders? World J Biol Chem 5:130-140.

Nagata K, Somura F, Obata K, Odashima M, Izawa H, Ichihara S, Nagasaka T, Iwase M, Yamada Y, and Nakashima N, et al. (2002) AT1 receptor blockade reduces cardiac calcineurin activity in hypertensive rats. Hypertension 40:168-174

Nakajima M, Hutchinson HG, Fujinaga M, Hayashida W, Morishita R, Zhang L, Horiuchi M, Pratt RE, and Dzau VJ (1995) The angiotensin II type 2 (AT2) receptor antagonizes the growth effects of the AT1 receptor: gain-of-function study using gene transfer. Proc Natl Acad Sci USA 92:10663-10667.

Nakajima M, Mukoyama M, Pratt RE, Horiuchi M, and Dzau VJ (1993) Cloning of cDNA and analysis of the gene for mouse angiotensin II type 2 receptor. Biochem Biophys Res Commun 197:393-399.

Nakamura S, Nakamura I, Ma L, Vaughan DE, and Fogo AB (2000) Plasminogen activator inhibitor-1 expression is regulated by the angiotensin type 1 receptor in vivo. Kidney Int 58:251-259.

Nakanishi M, Harada M, Kishimoto I, Kuwahara K, Kawakami R, Nakagawa Y, Yasuno S, Usami S, Kinoshita H, and Adachi Y, et al. (2007) Genetic disruption of angiotensin II type 1a receptor improves long-term survival of mice with chronic severe aortic regurgitation. Circulation 71:1310-1316

Nakashima H, Frank GD, Shirai H, Hinoki A, Higuchi S, Ohtsu H, Eguchi K, Sanjay A, Reyland ME, and Dempsey PJ, et al. (2008) Novel role of protein kinase C-delta Tyr 311 phosphorylation in vascular smooth muscle cell hypertrophy by angiotensin II. Hypertension 51:232-238.

Nakayama M, Yan X, Price RL, Borg TK, Ito K, Sanbe A, Robbins J, and Lorell BH (2005) Chronic ventricular myocyte-specific overexpression of angiotensin II type 2 receptor results in intrinsic myocyte contractile dysfunction. Am J Physiol Heart Circ Physiol 288:H317-H327.

Navarrete Santos A, Augustin R, Lazzari G, Galli C, Sreenan JM, and Fischer B (2000a) The insulin-dependent glucose transporter isoform 4 is expressed in bovine blastocysts. Biochem Biophys Res Commun 271:753-760.

Navarrete Santos A, Langner J, and Riemann D (2000b) Enzymatic activity is not a precondition for the intracellular calcium increase mediated by mAbs specific for aminopeptidase N/CD13. Adv Exp Med Biol 477:43-47.

Navarrete Santos A, Roentsch J, Danielsen EM, Langner J, and Riemann D (2000c) Aminopeptidase N/CD13 is associated with raft membrane microdomains in monocytes. Biochem Biophys Res Commun 269:143-148.

Naveri L, Stromberg C, and Saavedra JM (1994) Angiotensin IV reverses the acute cerebral blood flow reduction after experimental subarachnoid hemorrhage in the rat. J Cereb Blood Flow Metab 14:1096-1099.

Nguyen G, Delarue F, Burcklé C, Bouzhir L, Giller T, and Sraer JD (2002) Pivotal role of the renin/prorenin receptor in angiotensin II production and cellular responses to renin. J Clin Invest 109:1417-1427.

Nickenig G, Michaelsen F, Müller C, Berger A, Vogel T, Sachinidis A, Vetter H, and Böhm M (2002) Destabilization of AT(1) receptor mRNA by calreticulin. Circ Res 90:53-58.

Nickenig G, Sachinidis A, Michaelsen F, Böhm M, Seewald S, and Vetter H (1997) Upregulation of vascular angiotensin II receptor gene expression by low-density lipoprotein in vascular smooth muscle cells. Circulation 95:473-478.

Nickenig G, Strehlow K, Bäumer AT, Baudler S, Wassmann S, Sauer H, and Böhm M (2000) Negative feedback regulation of reactive oxygen species on AT1 receptor gene expression. Br J Pharmacol 131:795-803.

Nikiforovich GV, Mihalik B, Catt KJ, and Marshall GR (2005) Molecular mechanisms of constitutive activity: mutations at position 111 of the angiotensin AT1 receptor. $J$ Pept Res 66:236-248.

Nikolaou A, Stijlemans B, Laoui D, Schouppe E, Tran HT, Tourwe D, Chai SY, Vanderheyden PM, and Van Ginderachter JA (2014) Presence and regulation of insulin-regulated aminopeptidase in mouse macrophages. $J$ Renin Angiotensin Aldosterone Syst 15:466-470.

Nikolaou A, Van den Eynde I, Tourwé D, Vauquelin G, Tóth G, Mallareddy JR, Poglitsch M, Van Ginderachter JA, and Vanderheyden PM (2013) [3H]IVDE77, a novel radioligand with high affinity and selectivity for the insulin-regulated aminopeptidase. Eur J Pharmacol 702:93-102.

Nio Y, Matsubara H, Murasawa S, Kanasaki M, and Inada M (1995) Regulation of gene transcription of angiotensin II receptor subtypes in myocardial infarction. $J$ Clin Invest 95:46-54.

Nishida M, Ogushi M, Suda R, Toyotaka M, Saiki S, Kitajima N, Nakaya M, Kim KM, Ide T, and Sato Y, et al. (2011) Heterologous down-regulation of angiotensin type 1 receptors by purinergic $\mathrm{P} 2 \mathrm{Y} 2$ receptor stimulation through S-nitrosylation of NFkappaB. Proc Natl Acad Sci USA 108:6662-6667.

Noda K, Feng YH, Liu XP, Saad Y, Husain A, and Karnik SS (1996) The active state of the AT1 angiotensin receptor is generated by angiotensin II induction. Biochemistry 35:16435-16442.

Noda K, Saad Y, and Karnik SS (1995a) Interaction of Phe8 of angiotensin II with Lys199 and His256 of AT1 receptor in agonist activation. $J$ Biol Chem 270: 28511-28514.

Noda K, Saad Y, Kinoshita A, Boyle TP, Graham RM, Husain A, and Karnik SS (1995b) Tetrazole and carboxylate groups of angiotensin receptor antagonists bind to the same subsite by different mechanisms. J Biol Chem 270:2284-2289.

Noda M, Shibouta Y, Inada Y, Ojima M, Wada T, Sanada T, Kubo K, Kohara Y, Naka $\mathrm{T}$, and Nishikawa K (1993) Inhibition of rabbit aortic angiotensin II (AII) receptor by CV-11974, a new nonpeptide AII antagonist. Biochem Pharmacol 46:311-318.

Nomura S, Ito T, Yamamoto E, Sumigama S, Iwase A, Okada M, Shibata K, Ando H, Ino K, and Kikkawa F, et al. (2005) Gene regulation and physiological function of placental leucine aminopeptidase/oxytocinase during pregnancy. Biochim Biophys Acta 1751:19-25.

Nouet S, Amzallag N, Li JM, Louis S, Seitz I, Cui TX, Alleaume AM, Di Benedetto M, Boden C, and Masson M, et al. (2004) Trans-inactivation of receptor tyrosine kinases by novel angiotensin II AT2 receptor-interacting protein, ATIP. J Biol Chem 279:28989-28997.

Nouet S and Nahmias C (2000) Signal transduction from the angiotensin II AT2 receptor. Trends Endocrinol Metab 11:1-6.

Oakley RH, Laporte SA, Holt JA, Caron MG, and Barak LS (2000) Differential affinities of visual arrestin, beta arrestin1, and beta arrestin2 for G protein-coupled receptors delineate two major classes of receptors. J Biol Chem 275:17201-17210.

Ogawa S and Glass CK (2004) Factor XIIIA (cross)links AT1 receptors to atherosclerosis. Cell 119:313-314

Ogihara T, Asano T, Ando K, Chiba Y, Sakoda H, Anai M, Shojima N, Ono H, Onishi $\mathrm{Y}$, and Fujishiro M, et al. (2002) Angiotensin II-induced insulin resistance is associated with enhanced insulin signaling. Hypertension 40:872-879.

Ogihara T, Nagano M, Higaki J, Kohara K, and Mikami H (1995) Persistent inhibition of the pressor and aldosterone responses to angiotensin-II by TCV-116 in normotensive subjects. J Cardiovasc Pharmacol 26:490-494.

Ohashi H, Takagi H, Oh H, Suzuma K, Suzuma I, Miyamoto N, Uemura A, Watanabe D, Murakami T, and Sugaya T, et al. (2004) Phosphatidylinositol 3-kinase/Akt regulates angiotensin II-induced inhibition of apoptosis in microvascular endothelial cells by governing survivin expression and suppression of caspase-3 activity. Circ Res 94:785-793.

Ohkubo N, Matsubara H, Nozawa Y, Mori Y, Murasawa S, Kijima K, Maruyama K, Masaki H, Tsutumi Y, and Shibazaki Y, et al. (1997) Angiotensin type 2 receptors are reexpressed by cardiac fibroblasts from failing myopathic hamster hearts and inhibit cell growth and fibrillar collagen metabolism. Circulation 96:3954-3962.

Ohtsu H, Dempsey PJ, and Eguchi S (2006a) ADAMs as mediators of EGF receptor transactivation by $\mathrm{G}$ protein-coupled receptors. Am J Physiol Cell Physiol 291: C1-C10.

Ohtsu H, Dempsey PJ, Frank GD, Brailoiu E, Higuchi S, Suzuki H, Nakashima H, Eguchi K, and Eguchi S (2006b) ADAM17 mediates epidermal growth factor receptor transactivation and vascular smooth muscle cell hypertrophy induced by angiotensin II. Arterioscler Thromb Vasc Biol 26:e133-e137.

Ohtsu H, Suzuki H, Nakashima H, Dhobale S, Frank GD, Motley ED, and Eguchi S (2006c) Angiotensin II signal transduction through small GTP-binding proteins: mechanism and significance in vascular smooth muscle cells. Hypertension 48 $534-540$

Ohyama K, Yamano Y, Sano T, Nakagomi Y, Hamakubo T, Morishima I, and Inagami T (1995) Disulfide bridges in extracellular domains of angiotensin II receptor type IA. Regul Pept 57:141-147.

Ojima M, Igata H, Tanaka M, Sakamoto H, Kuroita T, Kohara Y, Kubo K, Fuse H, Imura Y, and Kusumoto K, et al. (2011) In vitro antagonistic properties of a new angiotensin type 1 receptor blocker, azilsartan, in receptor binding and function studies. J Pharmacol Exp Ther 336:801-808.

Ojima M, Inada Y, Shibouta Y, Wada T, Sanada T, Kubo K, and Nishikawa K (1997) Candesartan (CV-11974) dissociates slowly from the angiotensin AT1 receptor. Eur $J$ Pharmacol 319:137-146.

Olivares-Reyes JA, Shah BH, Hernández-Aranda J, García-Caballero A, Farshor MP, García-Sáinz JA, and Catt KJ (2005) Agonist-induced interactions between angiotensin AT1 and epidermal growth factor receptors. Mol Pharmacol 68: $356-364$

Olivares-Reyes JA, Smith RD, Hunyady L, Shah BH, and Catt KJ (2001) Agonistinduced signaling, desensitization, and internalization of a phosphorylationdeficient AT1A angiotensin receptor. J Biol Chem 276:37761-37768.

Oliveira L, Costa-Neto CM, Nakaie CR, Schreier S, Shimuta SI, and Paiva AC (2007) The angiotensin II AT1 receptor structure-activity correlations in the light of rhodopsin structure. Physiol Rev 87:565-592.

Oliverio MI, Kim HS, Ito M, Le T, Audoly L, Best CF, Hiller S, Kluckman K, Maeda $\mathrm{N}$, and Smithies O, et al. (1998) Reduced growth, abnormal kidney structure, and type 2 (AT2) angiotensin receptor-mediated blood pressure regulation in mice 
lacking both AT1A and AT1B receptors for angiotensin II. Proc Natl Acad Sci USA 95:15496-15501.

Olson ML, Olson EA, Qualls JH, Stratton JJ, Harding JW, and Wright JW (2004) Norleucine1-Angiotensin IV alleviates mecamylamine-induced spatial memory deficits. Peptides 25:233-241.

Omura T, Yoshiyama M, Ishikura F, Kobayashi H, Takeuchi K, Beppu S, and Yoshikawa J (2001) Myocardial ischemia activates the JAK-STAT pathway through angiotensin II signaling in in vivo myocardium of rats. J Mol Cell Cardiol 33:307-316.

Oppermann M, Diversé-Pierluissi M, Drazner MH, Dyer SL, Freedman NJ, Peppel KC, and Lefkowitz RJ (1996a) Monoclonal antibodies reveal receptor specificity among G-protein-coupled receptor kinases. Proc Natl Acad Sci USA 93:7649-7654.

Oppermann M, Freedman NJ, Alexander RW, and Lefkowitz RJ (1996b) Phosphorylation of the type $1 \mathrm{~A}$ angiotensin II receptor by $\mathrm{G}$ protein-coupled receptor kinases and protein kinase C. J Biol Chem 271:13266-13272.

Oppermann M, Gess B, Schweda F, and Castrop H (2010) Atrap deficiency increases arterial blood pressure and plasma volume. J Am Soc Nephrol 21:468-477.

Oro C, Qian H, and Thomas WG (2007) Type 1 angiotensin receptor pharmacology: signaling beyond G proteins. Pharmacol Ther 113:210-226.

Oshita A, Iwai M, Chen R, Ide A, Okumura M, Fukunaga S, Yoshii T, Mogi M, Higaki J, and Horiuchi M (2006) Attenuation of inflammatory vascular remodeling by angiotensin II type 1 receptor-associated protein. Hypertension 48:671-676.

Ott C, Titze SI, Schwarz TK, Kreutz R, Hilgers KF, Schmidt BM, Schlaich MP, and Schmieder RE (2007) High sodium intake modulates left ventricular mass in patients with $\mathrm{G}$ expression of $+1675 \mathrm{G} / \mathrm{A}$ angiotensin II receptor type 2 gene. $J$ Hypertens 25:1627-1632.

Pachori AS, Wang H, Gelband CH, Ferrario CM, Katovich MJ, and Raizada MK (2000) Inability to induce hypertension in normotensive rat expressing AT(1) receptor antisense. Circ Res 86:1167-1172.

Padia SH and Carey RM (2013) AT2 receptors: beneficial counter-regulatory role in cardiovascular and renal function. Pflugers Arch 465:99-110.

Page IH and Helmer OM (1940) Angiotonin-Activator, Renin- and AngiotoninInhibitor, and the Mechanism of Angiotonin Tachyphylaxis in Normal, Hypertensive, and Nephrectomized Animals. J Exp Med 71:495-519.

Pang J, Yan C, Natarajan K, Cavet ME, Massett MP, Yin G, and Berk BC (2008) GIT1 mediates HDAC5 activation by angiotensin II in vascular smooth muscle cells. Arterioscler Thromb Vasc Biol 28:892-898.

Papaiahgari S, Zhang Q, Kleeberger SR, Cho HY, and Reddy SP (2006) Hyperoxia stimulates an Nrf2-ARE transcriptional response via ROS-EGFR-PI3K-Akt/ERK MAP kinase signaling in pulmonary epithelial cells. Antioxid Redox Signal 8: 43-52.

Paradis P, Dali-Youcef N, Paradis FW, Thibault G, and Nemer M (2000) Overexpression of angiotensin II type I receptor in cardiomyocytes induces cardiac hypertrophy and remodeling. Proc Natl Acad Sci USA 97:931-936.

Parnot C, Bardin S, Miserey-Lenkei S, Guedin D, Corvol P, and Clauser E (2000) Systematic identification of mutations that constitutively activate the angiotensin II type $1 \mathrm{~A}$ receptor by screening a randomly mutated cDNA library with an original pharmacological bioassay. Proc Natl Acad Sci USA 97:7615-7620.

Paschos P and Tziomalos K (2012) Nonalcoholic fatty liver disease and the reninangiotensin system: Implications for treatment. World J Hepatol 4:327-331.

Passos-Silva DG, Verano-Braga T, and Santos RA (2013) Angiotensin-(1-7): beyond the cardio-renal actions. Clin Sci (Lond) 124:443-456.

Patel CB, Noor N, and Rockman HA (2010) Functional selectivity in adrenergic and angiotensin signaling systems. Mol Pharmacol 78:983-992.

Patel JM, Martens JR, Li YD, Gelband CH, Raizada MK, and Block ER (1998) Angiotensin IV receptor-mediated activation of lung endothelial NOS is associated with vasorelaxation. Am J Physiol 275:L1061-L1068.

Patel VB, Bodiga S, Fan D, Das SK, Wang Z, Wang W, Basu R, Zhong J, Kassiri Z, and Oudit GY (2012) Cardioprotective effects mediated by angiotensin II type 1 receptor blockade and enhancing angiotensin 1-7 in experimental heart failure in angiotensin-converting enzyme 2-null mice. Hypertension 59:1195-1203.

Patiag D, Qu X, Gray S, Idris I, Wilkes M, Seale JP, and Donnelly R (2000) Possible interactions between angiotensin II and insulin: effects on glucose and lipid metabolism in vivo and in vitro. J Endocrinol 167:525-531.

Paul M, Poyan Mehr A, and Kreutz R (2006) Physiology of local renin-angiotensin systems. Physiol Rev 86:747-803.

Peach MJ (1977) Renin-angiotensin system: biochemistry and mechanisms of action Physiol Rev 57:313-370.

Peach MJ and Levens NR (1980) Molecular approaches to the study of angiotensin receptors. Adv Exp Med Biol 130:171-194.

Peck GR, Ye S, Pham V, Fernando RN, Macaulay SL, Chai SY, and Albiston AL (2006) Interaction of the Akt substrate, AS160, with the glucose transporter 4 vesicle marker protein, insulin-regulated aminopeptidase. Mol Endocrinol 20: 2576-2583.

Pederson ES, Harding JW, and Wright JW (1998) Attenuation of scopolamineinduced spatial learning impairments by an angiotensin IV analog. Regul Pept 74: 97-103.

Peiró C, Vallejo S, Gembardt F, Azcutia V, Heringer-Walther S, Rodríguez-Mañas L, Schultheiss HP, Sánchez-Ferrer CF, and Walther T (2007) Endothelial dysfunction through genetic deletion or inhibition of the $\mathrm{G}$ protein-coupled receptor Mas: a new target to improve endothelial function. J Hypertens 25:2421-2425.

Peng H, Li W, Seth DM, Nair AR, Francis J, and Feng Y (2013) (Pro)renin receptor mediates both angiotensin II-dependent and -independent oxidative stress in neuronal cells. PLoS One 8:e58339.

Perbal B (2004) CCN proteins: multifunctional signalling regulators. Lancet 363: 62-64.

Pérodin J, Deraët M, Auger-Messier M, Boucard AA, Rihakova L, Beaulieu ME Lavigne P, Parent JL, Guillemette G, and Leduc R, et al. (2002) Residues 293 and 294 are ligand contact points of the human angiotensin type 1 receptor. Biochemistry 41:14348-14356.
Petracco RG, Kong A, Grechukhina O, Krikun G, and Taylor HS (2012) Global gene expression profiling of proliferative phase endometrium reveals distinct functional subdivisions. Reprod Sci 19:1138-1145.

Pfeffer MA and Braunwald E (1990) Ventricular remodeling after myocardial infarction. Experimental observations and clinical implications. Circulation 81: 1161-1172.

Pham V, Albiston AL, Downes CE, Wong CH, Diwakarla S, Ng L, Lee S, Crack PJ, and Chai SY (2012) Insulin-regulated aminopeptidase deficiency provides protection against ischemic stroke in mice. J Neurotrauma 29:1243-1248.

Pinaud F, Bocquet A, Dumont O, Retailleau K, Baufreton C, Andriantsitohaina R, Loufrani L, and Henrion D (2007) Paradoxical role of angiotensin II type 2 receptors in resistance arteries of old rats. Hypertension 50:96-102.

Pinheiro SV, Ferreira AJ, Kitten GT, da Silveira KD, da Silva DA, Santos SH, Gava E, Castro CH, Magalhães JA, and da Mota RK, et al. (2009) Genetic deletion of the angiotensin-(1-7) receptor Mas leads to glomerular hyperfiltration and microalbuminuria. Kidney Int 75:1184-1193.

Pinheiro SV, Simões e Silva AC, Sampaio WO, de Paula RD, Mendes EP, Bontempo ED, Pesquero JB, Walther T, Alenina N, and Bader M, et al. (2004) Nonpeptide AVE 0991 is an angiotensin-(1-7) receptor Mas agonist in the mouse kidney. $H y$ pertension 44:490-496.

Polte TR, Naftilan AJ, and Hanks SK (1994) Focal adhesion kinase is abundant in developing blood vessels and elevation of its phosphotyrosine content in vascular smooth muscle cells is a rapid response to angiotensin II. J Cell Biochem 55: 106-119.

Porrello ER, D'Amore A, Curl CL, Allen AM, Harrap SB, Thomas WG, and Delbridge LM (2009) Angiotensin II type 2 receptor antagonizes angiotensin II type 1 receptor-mediated cardiomyocyte autophagy. Hypertension 53:1032-1040.

Poupart ME, Fessart D, Cotton M, Laporte SA, and Claing A (2007) ARF6 regulates angiotensin II type 1 receptor endocytosis by controlling the recruitment of AP-2 and clathrin. Cell Signal 19:2370-2378.

Prenzel N, Zwick E, Daub H, Leserer M, Abraham R, Wallasch C, and Ullrich A (1999) EGF receptor transactivation by G-protein-coupled receptors requires metalloproteinase cleavage of proHB-EGF. Nature 402:884-888.

Ptasinska-Wnuk D, Kunert-Radek J, and Pawlikowski M (2003) Angiotensins II and IV stimulate the rat anterior pituitary cell proliferation independently of the AT1 receptor subtype. Neuroendocrinol Lett 24:397-400.

Pucell AG, Hodges JC, Sen I, Bumpus FM, and Husain A (1991) Biochemical properties of the ovarian granulosa cell type 2-angiotensin II receptor. Endocrinology 128:1947-1959.

Pueyo ME, Gonzalez W, Nicoletti A, Savoie F, Arnal JF, and Michel JB (2000) Angiotensin II stimulates endothelial vascular cell adhesion molecule-1 via nuclear factor-kappaB activation induced by intracellular oxidative stress. Arterioscler Thromb Vasc Biol 20:645-651.

Pulakat L, Gray A, Johnson J, Knowle D, Burns V, and Gavini N (2002) Role of C-terminal cytoplasmic domain of the AT2 receptor in ligand binding and signaling. FEBS Lett 524:73-78.

Pulakat L, Tadessee AS, Dittus JJ, and Gavini N (1998) Role of Lys215 located in the fifth transmembrane domain of the AT2 receptor in ligand-receptor interaction. Regul Pept 73:51-57.

Qian H, Pipolo L, and Thomas WG (1999) Identification of protein kinase C phosphorylation sites in the angiotensin II (AT1A) receptor. Biochem $J$ 343:637-644.

Qian H, Pipolo L, and Thomas WG (2001) Association of beta-Arrestin 1 with the type $1 \mathrm{~A}$ angiotensin II receptor involves phosphorylation of the receptor carboxyl terminus and correlates with receptor internalization. Mol Endocrinol 15:1706-1719.

Rabelo LA, Xu P, Todiras M, Sampaio WO, Buttgereit J, Bader M, Santos RA, and Alenina N (2008) Ablation of angiotensin (1-7) receptor Mas in C57Bl/6 mice causes endothelial dysfunction. J Am Soc Hypertens 2:418-424.

Rajagopal K, Lefkowitz RJ, and Rockman HA (2005) When 7 transmembrane receptors are not G protein-coupled receptors. J Clin Invest 115:2971-2974.

Rajagopalan S, Kurz S, Münzel T, Tarpey M, Freeman BA, Griendling KK, and Harrison DG (1996) Angiotensin II-mediated hypertension in the rat increases vascular superoxide production via membrane NADH/NADPH oxidase activation. Contribution to alterations of vasomotor tone. J Clin Invest 97:1916-1923.

Rakesh K, Yoo B, Kim IM, Salazar N, Kim KS, and Rockman HA (2010) betaArrestin-biased agonism of the angiotensin receptor induced by mechanical stress. Sci Signal 3:ra46.

Rakušan D, Bürgelová M, Vaněčková I, Vaňourková Z, Husková Z, Skaroupková $\mathrm{P}$ Mrázová I, Opočenský M, Kramer HJ, and Netuka I, et al. (2010) Knockout of angiotensin 1-7 receptor Mas worsens the course of two-kidney, one-clip Goldblatt hypertension: roles of nitric oxide deficiency and enhanced vascular responsiveness to angiotensin II. Kidney Blood Press Res 33:476-488.

Ramchandran R, Takezako T, Saad Y, Stull L, Fink B, Yamada H, Dikalov S, Harrison DG, Moravec C, and Karnik SS (2006) Angiotensinergic stimulation of vascular endothelium in mice causes hypotension, bradycardia, and attenuated angiotensin response. Proc Natl Acad Sci USA 103:19087-19092.

Re RN and Cook JL (2011) Noncanonical intracrine action. J Am Soc Hypertens 5: $435-448$

Reaves PY, Wang HW, Katovich MJ, Gelband CH, and Raizada MK (2000) Attenuation of hypertension by systemic delivery of retroviral vector containing type I angiotensin II receptor antisense cDNA. Methods 22:211-218.

Regenhardt RW, Bennion DM, and Sumners C (2014a) Cerebroprotective action of angiotensin peptides in stroke. Clin Sci (Lond) 126:195-205.

Regenhardt RW, Desland F, Mecca AP, Pioquinto DJ, Afzal A, Mocco J, and Sumners C (2013) Anti-inflammatory effects of angiotensin-(1-7) in ischemic stroke. Neuropharmacology 71:154-163.

Regenhardt RW, Mecca AP, Desland F, Ritucci-Chinni PF, Ludin JA, Greenstein D, Banuelos C, Bizon JL, Reinhard MK, and Sumners C (2014b) Centrally administered angiotensin-(1-7) increases the survival of stroke-prone spontaneously hypertensive rats. Exp Physiol 99:442-453. 
Regitz-Zagrosek V, Friedel N, Heymann A, Bauer P, Neuss M, Rolfs A, Steffen C, Hildebrandt A, Hetzer R, and Fleck E (1995) Regulation, chamber localization, and subtype distribution of angiotensin II receptors in human hearts. Circulation 91: 1461-1471.

Rehman A, Leibowitz A, Yamamoto N, Rautureau Y, Paradis P, and Schiffrin EL (2012) Angiotensin type 2 receptor agonist compound 21 reduces vascular injury and myocardial fibrosis in stroke-prone spontaneously hypertensive rats. Hypertension 59:291-299.

Reis AB, Araújo FC, Pereira VM, Dos Reis AM, Santos RA, and Reis FM (2010) Angiotensin (1-7) and its receptor Mas are expressed in the human testis: implications for male infertility. J Mol Histol 41:75-80.

Reis FM, Bouissou DR, Pereira VM, Camargos AF, dos Reis AM, and Santos RA (2011) Angiotensin-(1-7), its receptor Mas, and the angiotensin-converting enzyme type 2 are expressed in the human ovary. Fertil Steril 95:176-181.

Renieri A, Pescucci C, Longo I, Ariani F, Mari F, and Meloni I (2005) Non-syndromic $\mathrm{X}$-linked mental retardation: from a molecular to a clinical point of view. $J$ Cell Physiol 204:8-20.

Rice AS, Dworkin RH, McCarthy TD, Anand P, Bountra C, McCloud PI, Hill J, Cutter G, Kitson G, and Desem N, et al.; EMA401-003 study group (2014) EMA401, an orally administered highly selective angiotensin II type 2 receptor antagonist, as a novel treatment for postherpetic neuralgia: a randomised, double-blind, placebocontrolled phase 2 clinical trial. Lancet 383:1637-1647.

Riemekasten G, Philippe A, Näther M, Slowinski T, Müller DN, Heidecke H, Matucci-Cerinic M, Czirják L, Lukitsch I, and Becker M, et al. (2011) Involvement of functional autoantibodies against vascular receptors in systemic sclerosis. Ann Rheum Dis 70:530-536.

Rittel W, Iselin B, Kappeler H, Riniker B, and Schwyzer R (1957) Synthesis of a highly effective angiotensin II amide (L-asparagynyl-L-arginyl-L-valyl-L-tyrosylL-isoleucyl-L-histidyl-L-prolyl-L-phenylalanine. in German Helv Chim Acta 40: 614-624.

Rivard K, Grandy SA, Douillette A, Paradis P, Nemer M, Allen BG, and Fiset C (2011) Overexpression of type 1 angiotensin II receptors impairs excitationcontraction coupling in the mouse heart. Am J Physiol Heart Circ Physiol 301: H2018-H2027.

Roberts JM (2000) Angiotensin-1 receptor autoantibodies: A role in the pathogenesis of preeclampsia? Circulation 101:2335-2337.

Roberts KA, Krebs LT, Kramár EA, Shaffer MJ, Harding JW, and Wright JW (1995) Autoradiographic identification of brain angiotensin IV binding sites and differential c-Fos expression following intracerebroventricular injection of angiotensin II and IV in rats. Brain Res 682:13-21.

Rodríguez-Vita J, Sánchez-López E, Esteban V, Rupérez M, Egido J, and Ruiz-Ortega M (2005) Angiotensin II activates the Smad pathway in vascular smooth muscle cells by a transforming growth factor-beta-independent mechanism. Circulation 111:2509-2517.

Rogi T, Tsujimoto M, Nakazato H, Mizutani S, and Tomoda Y (1996) Human placental leucine aminopeptidase/oxytocinase. A new member of type II membranespanning zinc metallopeptidase family. J Biol Chem 271:56-61.

Ross SA, Scott HM, Morris NJ, Leung WY, Mao F, Lienhard GE, and Keller SR (1996) Characterization of the insulin-regulated membrane aminopeptidase in 3T3-L1 adipocytes. J Biol Chem 271:3328-3332.

Rowe BP, Kalivas PW, and Speth RC (1990) Autoradiographic localization of angiotensin II receptor binding sites on noradrenergic neurons of the locus coeruleus of the rat. $J$ Neurochem 55:533-540.

Ruan X, Oliverio MI, Coffman TM, and Arendshorst WJ (1999) Renal vascular reactivity in mice: AngII-induced vasoconstriction in AT1A receptor null mice. J Am Soc Nephrol 10:2620-2630.

Rubanyi GM and Vanhoutte PM (1986) Superoxide anions and hyperoxia inactivate endothelium-derived relaxing factor. Am J Physiol 250:H822-H827.

Ruiz-Ortega M, Lorenzo O, Rupérez M, Blanco J, and Egido J (2001a) Systemic infusion of angiotensin II into normal rats activates nuclear factor-kappaB and AP-1 in the kidney: role of AT(1) and AT(2) receptors. Am J Pathol 158:1743-1756.

Ruiz-Ortega M, Lorenzo O, Rupérez M, Esteban V, Suzuki Y, Mezzano S, Plaza JJ, and Egido $J$ (2001b) Role of the renin-angiotensin system in vascular diseases: expanding the field. Hypertension 38:1382-1387.

Ruiz-Ortega M, Lorenzo O, Rupérez M, König S, Wittig B, and Egido J (2000) Angiotensin II activates nuclear transcription factor kappaB through AT(1) and AT(2) in vascular smooth muscle cells: molecular mechanisms. Circ Res 86:1266-1272.

Rupérez M, Lorenzo O, Blanco-Colio LM, Esteban V, Egido J, and Ruiz-Ortega M (2003) Connective tissue growth factor is a mediator of angiotensin II-induced fibrosis. Circulation 108:1499-1505.

Sabe H, Hamaguchi M, and Hanafusa H (1997) Cell to substratum adhesion is involved in v-Src-induced cellular protein tyrosine phosphorylation: implication for the adhesion-regulated protein tyrosine phosphatase activity. Oncogene 14 1779-1788.

Sabri A, Govindarajan G, Griffin TM, Byron KL, Samarel AM, and Lucchesi PA (1998) Calcium- and protein kinase C-dependent activation of the tyrosine kinase PYK2 by angiotensin II in vascular smooth muscle. Circ Res 83:841-851.

Sadoshima J (1998) Versatility of the angiotensin II type 1 receptor. Circ Res $\mathbf{8 2}$ $1352-1355$

Sadoshima J and Izumo S (1996) The heterotrimeric G q protein-coupled angiotensin II receptor activates p21 ras via the tyrosine kinase-Shc-Grb2-Sos pathway in cardiac myocytes. EMBO J 15:775-787.

Sadoshima J, Xu Y, Slayter HS, and Izumo S (1993) Autocrine release of angiotensin II mediates stretch-induced hypertrophy of cardiac myocytes in vitro. Cell $\mathbf{7 5}$ : 977-984.

Saito Y and Berk BC (2001) Transactivation: a novel signaling pathway from angiotensin II to tyrosine kinase receptors. J Mol Cell Cardiol 33:3-7.

Salem S, Jankowski V, Asare Y, Liehn E, Welker P, Raya-Bermudez A, PinedaMartos C, Rodriguez M, Muñoz-Castañeda JR, and Bruck H, et al. (2015) Identification of the vasoconstriction-inhibiting factor (VIF), a potent endogenous cofactor of angiotensin II acting on the angiotensin II type 2 receptor. Circulation 131:1426-1434.

Sampaio WO, Henrique de Castro C, Santos RA, Schiffrin EL, and Touyz RM (2007a) Angiotensin-(1-7) counterregulates angiotensin II signaling in human endothelial cells. Hypertension 50:1093-1098.

Sampaio WO, Souza dos Santos RA, Faria-Silva R, da Mata Machado LT, Schiffrin EL, and Touyz RM (2007b) Angiotensin-(1-7) through receptor Mas mediates endothelial nitric oxide synthase activation via Akt-dependent pathways. Hypertension 49:185-192.

Sandberg K, Ji H, Clark AJ, Shapira H, and Catt KJ (1992) Cloning and expression of a novel angiotensin II receptor subtype. J Biol Chem 267:9455-9458.

Sandmann S, Spitznagel H, Chung O, Xia QG, Illner S, Jänichen G, Rossius B, Daemen MJ, and Unger T (1998) Effects of the calcium channel antagonist mibefradil on haemodynamic and morphological parameters in myocardial infarctioninduced cardiac failure in rats. Cardiovasc Res 39:339-350.

Santos EL, Pesquero JB, Oliveira L, Paiva AC, and Costa-Neto CM (2004a) Mutagenesis of the AT1 receptor reveals different binding modes of angiotensin II and [Sar1]-angiotensin II. Regul Pept 119:183-188.

Santos EL, Reis RI, Silva RG, Shimuta SI, Pecher C, Bascands JL, Schanstra JP, Oliveira L, Bader M, and Paiva AC, et al. (2007) Functional rescue of a defective angiotensin II AT1 receptor mutant by the Mas protooncogene. Regul Pept 141: 159-167.

Santos RA and Baracho NC (1992) Angiotensin-(1-7) is a potent antidiuretic peptide in rats. Braz J Med Biol Res 25:651-654.

Santos RA, Campagnole-Santos MJ, and Andrade SP (2000) Angiotensin-(1-7): an update. Regul Pept 91:45-62.

Santos RA, Campagnole-Santos MJ, Baracho NC, Fontes MA, Silva LC, Neves LA Oliveira DR, Caligiorne SM, Rodrigues AR, and Gropen Júnior C, et al (1994) Characterization of a new angiotensin antagonist selective for angiotensin-(1-7): evidence that the actions of angiotensin-(1-7) are mediated by specific angiotensin receptors. Brain Res Bull 35:293-298.

Santos RA, Castro CH, Gava E, Pinheiro SV, Almeida AP, Paula RD, Cruz JS, Ramos AS, Rosa KT, and Irigoyen MC, et al. (2006) Impairment of in vitro and in vivo heart function in angiotensin-(1-7) receptor MAS knockout mice. Hypertension 47: 996-1002.

Santos RA, Ferreira AJ, Nadu AP, Braga AN, de Almeida AP, Campagnole-Santos MJ, Baltatu O, Iliescu R, Reudelhuber TL, and Bader M (2004b) Expression of an angiotensin-(1-7)-producing fusion protein produces cardioprotective effects in rats. Physiol Genomics 17:292-299.

Santos RA, Ferreira AJ, Verano-Braga T, and Bader M (2013) Angiotensinconverting enzyme 2, angiotensin-(1-7) and Mas: new players of the reninangiotensin system. J Endocrinol 216:R1-R17.

Santos RA, Haibara AS, Campagnole-Santos MJ, Simões e Silva AC, Paula RD, Pinheiro SV, Leite MF, Lemos VS, Silva DM, and Guerra MT, et al. (2003a) Characterization of a new selective antagonist for angiotensin-(1-7), D-pro7angiotensin-(1-7). Hypertension 41:737-743.

Santos RA, Simoes e Silva AC, Maric C, Silva DM, Machado RP, de Buhr I, HeringerWalther S, Pinheiro SV, Lopes MT, and Bader M, et al. (2003b) Angiotensin-(1-7) is an endogenous ligand for the G protein-coupled receptor Mas. Proc Natl Acad Sci USA 100:8258-8263.

Santos SH, Fernandes LR, Mario EG, Ferreira AV, Pôrto LC, Alvarez-Leite JI, Botion LM, Bader M, Alenina N, and Santos RA (2008) Mas deficiency in FVB/N mice produces marked changes in lipid and glycemic metabolism. Diabetes 57: 340-347.

Sanz-Rosa D, Oubiña MP, Cediel E, de Las Heras N, Vegazo O, Jiménez J, Lahera V, and Cachofeiro V (2005) Effect of AT1 receptor antagonism on vascular and circulating inflammatory mediators in SHR: role of NF-kappaB/IkappaB system. Am $J$ Physiol Heart Circ Physiol 288:H111-H115.

Sardinia MF, Hanesworth JM, Krebs LT, and Harding JW (1993) AT4 receptor binding characteristics: D-amino acid- and glycine-substituted peptides. Peptides 14:949-954.

Sardinia MF, Hanesworth JM, Krishnan F, and Harding JW (1994) AT4 receptor structure-binding relationship: N-terminal-modified angiotensin IV analogues. Peptides 15:1399-1406.

Sarkis A, Lopez B, and Roman RJ (2004) Role of 20-hydroxyeicosatetraenoic acid and epoxyeicosatrienoic acids in hypertension. Curr Opin Nephrol Hypertens 13: $205-214$.

Sasaki K, Murohara T, Ikeda H, Sugaya T, Shimada T, Shintani S, and Imaizumi T (2002) Evidence for the importance of angiotensin II type 1 receptor in ischemiainduced angiogenesis. J Clin Invest 109:603-611.

Sasaki K, Yamano Y, Bardhan S, Iwai N, Murray JJ, Hasegawa M, Matsuda Y, and Inagami T (1991) Cloning and expression of a complementary DNA encoding a bovine adrenal angiotensin II type-1 receptor. Nature 351:230-233.

Sasamura H, Hein L, Krieger JE, Pratt RE, Kobilka BK, and Dzau VJ (1992) Cloning, characterization, and expression of two angiotensin receptor (AT-1) isoforms from the mouse genome. Biochem Biophys Res Commun 185:253-259.

Sasamura H, Shimizu-Hirota R, Nakaya H, and Saruta T (2001) Effects of AT1 receptor antagonist on proteoglycan gene expression in hypertensive rats. Hypertens Res 24:165-172.

Saulière A, Bellot M, Paris H, Denis C, Finana F, Hansen JT, Altié MF, Seguelas MH, Pathak A, and Hansen JL, et al. (2012) Deciphering biased-agonism complexity reveals a new active AT1 receptor entity. Nat Chem Biol 8:622-630.

Savergnini SQ, Beiman M, Lautner RQ, de Paula-Carvalho V, Allahdadi K, Pessoa DC, Costa-Fraga FP, Fraga-Silva RA, Cojocaru G, and Cohen Y, et al. (2010) Vascular relaxation, antihypertensive effect, and cardioprotection of a novel peptide agonist of the MAS receptor. Hypertension 56:112-120.

Savergnini SQ, Ianzer D, Carvalho MB, Ferreira AJ, Silva GA, Marques FD, Peluso AA, Beiman M, Cojocaru G, and Cohen Y, et al. (2013) The novel Mas agonist CGEN-856S, attenuates isoproterenol-induced cardiac remodeling and myocardial infarction injury in rats. PLoS One 8:e57757. 
Sayeski PP, Ali MS, Harp JB, Marrero MB, and Bernstein KE (1998) Phosphorylation of p130Cas by angiotensin II is dependent on c-Src, intracellular $\mathrm{Ca} 2+$, and protein kinase C. Circ Res 82:1279-1288.

Schäfer B, Marg B, Gschwind A, and Ullrich A (2004) Distinct ADAM metalloproteinases regulate $\mathrm{G}$ protein-coupled receptor-induced cell proliferation and survival. J Biol Chem 279:47929-47938.

Schambye HT, Hjorth SA, Bergsma DJ, Sathe G, and Schwartz TW (1994) Differentiation between binding sites for angiotensin II and nonpeptide antagonists on the angiotensin II type 1 receptors. Proc Natl Acad Sci USA 91:7046-7050.

Schena M, Mulatero P, Schiavone D, Mengozzi G, Tesio L, Chiandussi L, and Veglio F (1999) Vasoactive hormones induce nitric oxide synthase mRNA expression and nitric oxide production in human endothelial cells and monocytes. Am J Hypertens 12:388-397.

Schieffer B, Schieffer E, Hilfiker-Kleiner D, Hilfiker A, Kovanen PT, Kaartinen M, Nussberger J, Harringer W, and Drexler H (2000) Expression of angiotensin II and interleukin 6 in human coronary atherosclerotic plaques: potential implications for inflammation and plaque instability. Circulation 101:1372-1378.

Schmidt B and Schieffer B (2003) Angiotensin II AT1 receptor antagonists. Clinical implications of active metabolites. J Med Chem 46:2261-2270.

Schmieder RE, Erdmann J, Delles C, Jacobi J, Fleck E, Hilgers K, and RegitzZagrosek V (2001) Effect of the angiotensin II type 2-receptor gene (+1675 G/A) on left ventricular structure in humans. J Am Coll Cardiol 37:175-182.

Schneider AW, Kalk JF, and Klein CP (1999) Effect of losartan, an angiotensin II receptor antagonist, on portal pressure in cirrhosis. Hepatology 29:334-339.

Schorb W, Booz GW, Dostal DE, Conrad KM, Chang KC, and Baker KM (1993) Angiotensin II is mitogenic in neonatal rat cardiac fibroblasts. Circ Res 72 $1245-1254$

Schultz J, Witt SA, Glascock BJ, Nieman ML, Reiser PJ, Nix SL, Kimball TR, and Doetschman T (2002) TGF-beta1 mediates the hypertrophic cardiomyocyte growth induced by angiotensin II. J Clin Invest 109:787-796.

Seachrist JL, Laporte SA, Dale LB, Babwah AV, Caron MG, Anborgh PH, and Ferguson SS (2002) Rab5 association with the angiotensin II type 1A receptor promotes Rab5 GTP binding and vesicular fusion. J Biol Chem 277:679-685.

Seals DF and Courtneidge SA (2003) The ADAMs family of metalloproteases: multidomain proteins with multiple functions. Genes Dev 17:7-30.

Seki Y, Kai H, Shibata R, Nagata T, Yasukawa H, Yoshimura A, and Imaizumi T (2000) Role of the JAK/STAT pathway in rat carotid artery remodeling after vascular injury. Circ Res 87:12-18.

Seko T, Ito M, Kureishi Y, Okamoto R, Moriki N, Onishi K, Isaka N, Hartshorne DJ, and Nakano T (2003) Activation of RhoA and inhibition of myosin phosphatase as important components in hypertension in vascular smooth muscle. Circ Res 92: 411-418.

Semple PF, Boyd AS, Dawes PM, and Morton JJ (1976) Angiotensin II and its heptapeptide (2-8), hexapeptide (3-8), and pentapeptide (4-8) metabolites in arterial and venous blood of man. Circ Res 39:671-678.

Sen CK and Packer L (1996) Antioxidant and redox regulation of gene transcription. FASEB J 10:709-720.

Senbonmatsu T, Ichihara S, Price E Jr, Gaffney FA, and Inagami T (2000) Evidence for angiotensin II type 2 receptor-mediated cardiac myocyte enlargement during in vivo pressure overload. J Clin Invest 106:R25-R29.

Senbonmatsu T, Saito T, Landon EJ, Watanabe O, Price E Jr, Roberts RL, Imboden H, Fitzgerald TG, Gaffney FA, and Inagami T (2003) A novel angiotensin II type 2 receptor signaling pathway: possible role in cardiac hypertrophy. EMBO J 22: $6471-6482$.

Seshiah PN, Weber DS, Rocic P, Valppu L, Taniyama Y, and Griendling KK (2002) Angiotensin II stimulation of $\mathrm{NAD}(\mathrm{P}) \mathrm{H}$ oxidase activity: upstream mediators. Circ Res 91:406-413.

Seta K, Nanamori M, Modrall JG, Neubig RR, and Sadoshima J (2002) AT1 receptor mutant lacking heterotrimeric $G$ protein coupling activates the Src-Ras-ERK pathway without nuclear translocation of ERKs. J Biol Chem 277:9268-9277.

Seta K and Sadoshima J (2003) Phosphorylation of tyrosine 319 of the angiotensin II type 1 receptor mediates angiotensin II-induced trans-activation of the epidermal growth factor receptor. J Biol Chem 278:9019-9026.

Sevá Pessôa B, van der Lubbe N, Verdonk K, Roks AJ, Hoorn EJ, and Danser AH (2013) Key developments in renin-angiotensin-aldosterone system inhibition. Nat Rev Nephrol 9:26-36.

Shah BH, Alberto Olivares-Reyes J, Yesilkaya A, and Catt KJ (2002) Independence of angiotensin II-induced MAP kinase activation from angiotensin type 1 receptor internalization in clone 9 hepatocytes. Mol Endocrinol 16:610-620.

Shah BH, Yesilkaya A, Olivares-Reyes JA, Chen HD, Hunyady L, and Catt KJ (2004) Differential pathways of angiotensin II-induced extracellularly regulated kinase 1 / 2 phosphorylation in specific cell types: role of heparin-binding epidermal growth factor. Mol Endocrinol 18:2035-2048.

Shalamanova L, Wilkinson MC, McArdle F, Jackson MJ, and Rustom R (2010) Characterisation of the expression of the Renin-Angiotensin system in primary and immortalised human renal proximal tubular cells. Nephron, Exp Nephrol 116:e53-e61.

Shanmugam S, Llorens-Cortes C, Clauser E, Corvol P, and Gasc JM (1995) Expression of angiotensin II AT2 receptor mRNA during development of rat kidney and adrenal gland. Am J Physiol 268:F922-F930.

Shemesh R, Toporik A, Levine Z, Hecht I, Rotman G, Wool A, Dahary D, Gofer E, Kliger Y, and Soffer MA, et al. (2008) Discovery and validation of novel peptide agonists for G-protein-coupled receptors. J Biol Chem 283:34643-34649.

Shirai H, Takahashi K, Katada T, and Inagami T (1995) Mapping of G protein coupling sites of the angiotensin II type 1 receptor. Hypertension 25:726-730.

Shukla AK, Violin JD, Whalen EJ, Gesty-Palmer D, Shenoy SK, and Lefkowitz RJ (2008) Distinct conformational changes in beta-arrestin report biased agonism at seven-transmembrane receptors. Proc Natl Acad Sci USA 105:9988-9993.

Shyu KG, Chen CC, Wang BW, and Kuan P (2001) Angiotensin II receptor antagonist blocks the expression of connexin43 induced by cyclical mechanical stretch in cultured neonatal rat cardiac myocytes. J Mol Cell Cardiol 33:691-698.
Sikkema JM, van Rijn BB, Franx A, Bruinse HW, de Roos R, Stroes ES, and van Faassen EE (2001) Placental superoxide is increased in pre-eclampsia. Placenta 22:304-308.

Silva LC, Fontes MA, Campagnole-Santos MJ, Khosla MC, Campos RR Jr, Guertzenstein PG, and Santos RA (1993) Cardiovascular effects produced by micro-injection of angiotensin-(1-7) on vasopressor and vasodepressor sites of the ventrolateral medulla. Brain Res 613:321-325.

Singh A, Boyer JL, Der CJ, and Zohn IE (2010a) Transformation by a nucleotideactivated P2Y receptor is mediated by activation of Galphai, Galphaq and Rhodependent signaling pathways. J Mol Signal 5:11.

Singh T, Singh K, and Sharma PL (2010b) Ameliorative potential of angiotensin1-7/ Mas receptor axis in streptozotocin-induced diabetic nephropathy in rats. Methods Find Exp Clin Pharmacol 32:19-25.

Singh VP, Le B, Bhat VB, Baker KM, and Kumar R (2007) High-glucose-induced regulation of intracellular ANG II synthesis and nuclear redistribution in cardiac myocytes. Am J Physiol Heart Circ Physiol 293:H939-H948.

Sipahi S, Hur E, Demirtas S, Kocayigit I, Bozkurt D, Tamer A, Gunduz H, and Duman S (2011) Body composition monitor measurement technique for the detection of volume status in peritoneal dialysis patients: the effect of abdominal fullness. Int Urol Nephrol 43:1195-1199.

Siragy HM (2000) The role of the AT2 receptor in hypertension. Am J Hypertens 13: $62 \mathrm{~S}-67 \mathrm{~S}$

Siragy HM (2010) The angiotensin II type 2 receptor and the kidney. J Renin Angiotensin Aldosterone Syst 11:33-36.

Siragy HM and Carey RM (1996) The subtype-2 (AT2) angiotensin receptor regulates renal cyclic guanosine $3^{\prime}, 5^{\prime}$-monophosphate and AT1 receptor-mediated prostaglandin E2 production in conscious rats. J Clin Invest 97:1978-1982.

Siragy HM, Inagami T, Ichiki T, and Carey RM (1999) Sustained hypersensitivity to angiotensin II and its mechanism in mice lacking the subtype-2 (AT2) angiotensin receptor. Proc Natl Acad Sci USA 96:6506-6510.

Siragy HM, Jaffa AA, Margolius HS, and Carey RM (1996) Renin-angiotensin system modulates renal bradykinin production. Am J Physiol 271:R1090-R1095.

Sironi L, Calvio AM, Arnaboldi L, Corsini A, Parolari A, de Gasparo M, Tremoli E, and Mussoni L (2001) Effect of valsartan on angiotensin II-induced plasminogen activator inhibitor-1 biosynthesis in arterial smooth muscle cells. Hypertension $\mathbf{3 7}$ 961-966.

Skeggs LT Jr, Lentz KE, Kahn JR, Shumway NP, and Woods KR (1956) The amino acid sequence of hypertensin. II. J Exp Med 104:193-197.

Skurk T, Lee YM, and Hauner H (2001) Angiotensin II and its metabolites stimulate PAI-1 protein release from human adipocytes in primary culture. Hypertension 37: 1336-1340.

Skurk T, van Harmelen V, and Hauner H (2004) Angiotensin II stimulates the release of interleukin- 6 and interleukin- 8 from cultured human adipocytes by activation of NF-kappaB. Arterioscler Thromb Vasc Biol 24:1199-1203.

Smith MT, Lau T, Wallace VC, Wyse BD, and Rice AS (2014) Analgesic efficacy of small-molecule angiotensin II type 2 receptor antagonists in a rat model of antiretroviral toxic polyneuropathy. Behav Pharmacol 25:137-146.

Smith MT, Woodruff TM, Wyse BD, Muralidharan A, and Walther T (2013a) A small molecule angiotensin II type 2 receptor $\left(\mathrm{AT}_{2} \mathrm{R}\right)$ antagonist produces analgesia in a rat model of neuropathic pain by inhibition of p38 mitogen-activated protein kinase (MAPK) and p44/p42 MAPK activation in the dorsal root ganglia. Pain Med 14:1557-1568.

Smith MT, Wyse BD, and Edwards SR (2013b) Small molecule angiotensin II type 2 receptor $\left(\mathrm{AT}_{2} \mathrm{R}\right)$ antagonists as novel analgesics for neuropathic pain: comparative pharmacokinetics, radioligand binding, and efficacy in rats. Pain Med 14:692-705. Smith NJ, Chan HW, Qian H, Bourne AM, Hannan KM, Warner FJ, Ritchie RH, Pearson RB, Hannan RD, and Thomas WG (2011) Determination of the exact molecular requirements for type 1 angiotensin receptor epidermal growth factor receptor transactivation and cardiomyocyte hypertrophy. Hypertension 57:973-980

Smith NJ and Luttrell LM (2006) Signal switching, crosstalk, and arrestin scaffolds: novel G protein-coupled receptor signaling in cardiovascular disease. Hypertension 48:173-179.

Smith RD, Baukal AJ, Zolyomi A, Gaborik Z, Hunyady L, Sun L, Zhang M, Chen HC, and Catt KJ (1998a) Agonist-induced phosphorylation of the endogenous AT1 angiotensin receptor in bovine adrenal glomerulosa cells. Mol Endocrinol 12:634-644.

Smith RD, Hunyady L, Olivares-Reyes JA, Mihalik B, Jayadev S, and Catt KJ (1998b) Agonist-induced phosphorylation of the angiotensin AT1a receptor is localized to a serine/threonine-rich region of its cytoplasmic tail. Mol Pharmacol 54:935-941.

Somsel Rodman J and Wandinger-Ness A (2000) Rab GTPases coordinate endocytosis. J Cell Sci 113:183-192.

Song H, Stevens CF, and Gage FH (2002) Astroglia induce neurogenesis from adult neural stem cells. Nature 417:39-44.

Sookoian S, Fernández MA, and Castaño G (2005) Effects of six months losartan administration on liver fibrosis in chronic hepatitis $\mathrm{C}$ patients: a pilot study. World $J$ Gastroenterol 11:7560-7563.

Souza AP, Sobrinho DB, Almeida JF, Alves GM, Macedo LM, Porto JE, Vencio EF, Colugnati DB, Dos Santos RA, and Ferreira AJ, et al. (2013) Angiotensin II Type 1 receptor blockade restores angiotensin-(1-7)-induced coronary vasodilation in hypertrophic rat hearts. Clin Sci 125:449-459

Speth RC and Kim KH (1990) Discrimination of two angiotensin II receptor subtypes with a selective agonist analogue of angiotensin II, p-aminophenylalanine6 angiotensin II. Biochem Biophys Res Commun 169:997-1006.

Speth RC, Rowe BP, Grove KL, Carter MR, and Saylor D (1991) Sulfhydryl reducing agents distinguish two subtypes of angiotensin II receptors in the rat brain. Brain Res 548:1-8.

Spiering W, Kroon AA, Fuss-Lejeune MM, Daemen MJ, and de Leeuw PW (2000) Angiotensin II sensitivity is associated with the angiotensin II type 1 receptor $\mathrm{A}(1166)$ $\mathrm{C}$ polymorphism in essential hypertensives on a high sodium diet. Hypertension 36 : 411-416. 
Steckelings UM, Kaschina E, and Unger T (2005) The AT2 receptor-a matter of love and hate. Peptides 26:1401-1409.

Steckelings UM, Larhed M, Hallberg A, Widdop RE, Jones ES, Wallinder C, Namsolleck P, Dahlöf B, and Unger T (2011a) Non-peptide AT2-receptor agonists. Curr Opin Pharmacol 11:187-192.

Steckelings UM, Paulis L, Unger T, and Bader M (2011b) Emerging drugs which target the renin-angiotensin-aldosterone system. Expert Opin Emerg Drugs 16: $619-630$.

Stegbauer J and Coffman TM (2011) New insights into angiotensin receptor actions: from blood pressure to aging. Curr Opin Nephrol Hypertens 20:84-88.

Stegbauer J, Potthoff SA, Quack I, Mergia E, Clasen T, Friedrich S, Vonend O, Woznowski M, Königshausen E, and Sellin L, et al. (2011) Chronic treatment with angiotensin-(1-7) improves renal endothelial dysfunction in apolipoproteinEdeficient mice. $\mathrm{Br} J$ Pharmacol 163:974-983.

Stoll M, Steckelings UM, Paul M, Bottari SP, Metzger R, and Unger T (1995) The angiotensin AT2-receptor mediates inhibition of cell proliferation in coronary endothelial cells. J Clin Invest 95:651-657.

Stragier B, Clinckers R, Meurs A, De Bundel D, Sarre S, Ebinger G, Michotte Y, and Smolders I (2006) Involvement of the somatostatin-2 receptor in the anticonvulsant effect of angiotensin IV against pilocarpine-induced limbic seizures in rats. J Neurochem 98:1100-1113.

Stragier B, De Bundel D, Sarre S, Smolders I, Vauquelin G, Dupont A, Michotte Y, and Vanderheyden P (2008) Involvement of insulin-regulated aminopeptidase in the effects of the renin-angiotensin fragment angiotensin IV: a review. Heart Fail Rev 13:321-337.

Stragier B, Demaegdt H, De Bundel D, Smolders I, Sarre S, Vauquelin G, Ebinger G, Michotte Y, and Vanderheyden P (2007) Involvement of insulin-regulated aminopeptidase and/or aminopeptidase $\mathrm{N}$ in the angiotensin IV-induced effect on dopamine release in the striatum of the rat. Brain Res 1131:97-105.

Stragier B, Sarre S, Vanderheyden P, Vauquelin G, Fournié-Zaluski MC, Ebinger G, and Michotte Y (2004) Metabolism of angiotensin II is required for its in vivo effect on dopamine release in the striatum of the rat. $J$ Neurochem 90:1251-1257.

Stroth U, Blume A, Mielke K, and Unger T (2000) Angiotensin AT(2) receptor stimulates ERK1 and ERK2 in quiescent but inhibits ERK in NGF-stimulated PC12W cells. Brain Res Mol Brain Res 78:175-180.

Stroth U, Meffert S, Gallinat S, and Unger T (1998) Angiotensin II and NGF differentially influence microtubule proteins in PC12W cells: role of the AT2 receptor. Brain Res Mol Brain Res 53:187-195.

$\mathrm{Su}$ JB (2014) Different cross-talk sites between the renin-angiotensin and the kallikrein-kinin systems. J Renin Angiotensin Aldosterone Syst 15:319-328.

Subramanian H, Kashem SW, Collington SJ, Qu H, Lambris JD, and Ali H (2011) PMX-53 as a dual CD88 antagonist and an agonist for Mas-related gene 2 (MrgX2) in human mast cells. Mol Pharmacol 79:1005-1013.

Sugaya T, Nishimatsu S, Tanimoto K, Takimoto E, Yamagishi T, Imamura K, Goto S, Imaizumi K, Hisada Y, and Otsuka A, et al. (1995) Angiotensin II type 1a receptor-deficient mice with hypotension and hyperreninemia. J Biol Chem $\mathbf{2 7 0}$ 18719-18722.

Sugden PH and Clerk A (1997) Regulation of the ERK subgroup of MAP kinase cascades through G protein-coupled receptors. Cell Signal 9:337-351.

Sugino H, Ozono R, Kurisu S, Matsuura H, Ishida M, Oshima T, Kambe M, Teranishi $\mathrm{Y}$, Masaki H, and Matsubara H (2001) Apoptosis is not increased in myocardium overexpressing type 2 angiotensin II receptor in transgenic mice. Hypertension $\mathbf{3 7}$ $1394-1398$

Sumners C and Myers LM (1991) Angiotensin II decreases cGMP levels in neuronal cultures from rat brain. Am J Physiol 260:C79-C87.

Sumners C, Tang W, Zelezna B, and Raizada MK (1991) Angiotensin II receptor subtypes are coupled with distinct signal-transduction mechanisms in neurons and astrocytes from rat brain. Proc Natl Acad Sci USA 88:7567-7571.

Suzuki H and Eguchi S (2006) Growth factor receptor transactivation in mediating end organ damage by angiotensin II. Hypertension 47:339-340.

Suzuki H, Motley ED, Frank GD, Utsunomiya H, and Eguchi S (2005) Recent progress in signal transduction research of the angiotensin II type-1 receptor: protein kinases, vascular dysfunction and structural requirement. Curr Med Chem Cardiovasc Hematol Agents 3:305-322.

Suzuki J, Matsubara H, Urakami M, and Inada M (1993) Rat angiotensin II (type 1A) receptor mRNA regulation and subtype expression in myocardial growth and hypertrophy. Circ Res 73:439-447.

Swanson GN, Hanesworth JM, Sardinia MF, Coleman JK, Wright JW, Hall KL, Miller-Wing AV, Stobb JW, Cook VI, and Harding EC, et al. (1992) Discovery of a distinct binding site for angiotensin II (3-8), a putative angiotensin IV receptor. Regul Pept 40:409-419.

Taguchi K, Kobayashi T, Takenouchi Y, Matsumoto T, and Kamata K (2011) Angiotensin II causes endothelial dysfunction via the GRK2/Akt/eNOS pathway in aortas from a murine type 2 diabetic model. Pharmacol Res 64:535-546.

Takahashi S and Mendelsohn ME (2003) Synergistic activation of endothelial nitricoxide synthase (eNOS) by HSP90 and Akt: calcium-independent eNOS activation involves formation of an HSP90-Akt-CaM-bound eNOS complex. J Biol Chem 278 30821-30827.

Takezako T, Gogonea C, Saad Y, Noda K, and Karnik SS (2004) "Network leaning" as a mechanism of insurmountable antagonism of the angiotensin II type 1 receptor by non-peptide antagonists. J Biol Chem 279:15248-15257.

Tallant EA, Ferrario CM, and Gallagher PE (2005) Angiotensin-(1-7) inhibits growth of cardiac myocytes through activation of the mas receptor. Am J Physiol Heart Circ Physiol 289:H1560-H1566.

Tamarat R, Silvestre JS, Durie M, and Levy BI (2002) Angiotensin II angiogenic effect in vivo involves vascular endothelial growth factor- and inflammationrelated pathways. Lab Invest 82:747-756.

Tamura K, Tanaka Y, Tsurumi Y, Azuma K, Shigenaga A, Wakui H, Masuda S, and Matsuda M (2007) The role of angiotensin AT1 receptor-associated protein in renin-angiotensin system regulation and function. Curr Hypertens Rep 9:121-127.
Tanaka M, Chen Z, Bartunkova S, Yamasaki N, and Izumo S (1999) The cardiac homeobox gene $\mathrm{Csx} / \mathrm{Nkx} 2.5$ lies genetically upstream of multiple genes essential for heart development. Development 126:1269-1280.

Tanaka M, Nanba D, Mori S, Shiba F, Ishiguro H, Yoshino K, Matsuura N, and Higashiyama S (2004) ADAM binding protein Eve-1 is required for ectodomain shedding of epidermal growth factor receptor ligands. $J$ Biol Chem 279: $41950-41959$.

Tanaka Y, Tamura K, Koide Y, Sakai M, Tsurumi Y, Noda Y, Umemura M, Ishigami T, Uchino K, and Kimura K, et al. (2005) The novel angiotensin II type 1 receptor (AT1R)-associated protein ATRAP downregulates AT1R and ameliorates cardiomyocyte hypertrophy. FEBS Lett 579:1579-1586.

Tang H, Zhao ZJ, Landon EJ, and Inagami T (2000) Regulation of calcium-sensitive tyrosine kinase Pyk2 by angiotensin II in endothelial cells. Roles of Yes tyrosine kinase and tyrosine phosphatase SHP-2. J Biol Chem 275:8389-8396.

Taniyama Y and Griendling KK (2003) Reactive oxygen species in the vasculature: molecular and cellular mechanisms. Hypertension 42:1075-1081.

Taniyama Y, Hitomi H, Shah A, Alexander RW, and Griendling KK (2005) Mechanisms of reactive oxygen species-dependent downregulation of insulin receptor substrate-1 by angiotensin II. Arterioscler Thromb Vasc Biol 25:1142-1147.

Taniyama Y, Ushio-Fukai M, Hitomi H, Rocic P, Kingsley MJ, Pfahnl C, Weber DS, Alexander RW, and Griendling KK (2004) Role of p38 MAPK and MAPKAPK-2 in angiotensin II-induced Akt activation in vascular smooth muscle cells. Am J Physiol Cell Physiol 287:C494-C499.

Taniyama Y, Weber DS, Rocic P, Hilenski L, Akers ML, Park J, Hemmings BA Alexander RW, and Griendling KK (2003) Pyk2- and Src-dependent tyrosine phosphorylation of PDK1 regulates focal adhesions. Mol Cell Biol 23:8019-8029.

Tassone EJ, Sciacqua A, Andreozzi F, Presta I, Perticone M, Carnevale D, Casaburo M, Hribal ML, Sesti G, and Perticone F (2013) Angiotensin (1-7) counteracts the negative effect of angiotensin II on insulin signalling in HUVECs. Cardiovasc Res 99:129-136.

Tatemoto K, Nozaki Y, Tsuda R, Konno S, Tomura K, Furuno M, Ogasawara H, Edamura K Takagi $\mathrm{H}$, and Iwamura $\mathrm{H}$, et al (2006) Immunoglobulin E-independent activation of mast cell is mediated by Mrg receptors. Biochem Biophys Res Commun 349:1322-1328.

Tchekalarova J, Kambourova T, and Georgiev V (2001) Effects of angiotensin III and angiotensin IV on pentylenetetrazol seizure susceptibility (threshold and kindling): interaction with adenosine A(1) receptors. Brain Res Bull 56:87-91.

Teerlink JR (1996) Neurohumoral mechanisms in heart failure: a central role for the renin-angiotensin system. J Cardiovasc Pharmacol 27 (Suppl 2):S1-S8.

Than A, Leow MK, and Chen P (2013) Control of adipogenesis by the autocrine interplays between angiotensin 1-7/Mas receptor and angiotensin II/AT1 receptor signaling pathways. J Biol Chem 288:15520-15531.

Thibault G, Lacombe MJ, Schnapp LM, Lacasse A, Bouzeghrane F, and Lapalme G (2001) Upregulation of alpha(8)beta(1)-integrin in cardiac fibroblast by angiotensin II and transforming growth factor-beta1. Am $J$ Physiol Cell Physiol 281: C1457-C1467.

Thomas WG (1999) Regulation of angiotensin II type 1 (AT1) receptor function. Regul Pept 79:9-23.

Thomas WG, Baker KM, Motel TJ, and Thekkumkara TJ (1995) Angiotensin II receptor endocytosis involves two distinct regions of the cytoplasmic tail. A role for residues on the hydrophobic face of a putative amphipathic helix. J Biol Chem 270: 22153-22159.

Thomas WG, Brandenburger Y, Autelitano DJ, Pham T, Qian H, and Hannan RD (2002) Adenoviral-directed expression of the type 1A angiotensin receptor promotes cardiomyocyte hypertrophy via transactivation of the epidermal growth factor receptor. Circ Res 90:135-142.

Thomas WG and Mendelsohn FA (2003) Angiotensin receptors: form and function and distribution. Int $J$ Biochem Cell Biol 35:774-779.

Thomas WG, Motel TJ, Kule CE, Karoor V, and Baker KM (1998) Phosphorylation of the angiotensin II (AT1A) receptor carboxyl terminus: a role in receptor endocytosis. Mol Endocrinol 12:1513-1524.

Thomas WG and Qian H (2003) Arresting angiotensin type 1 receptors. Trends Endocrinol Metab 14:130-136.

Thomas WG, Qian H, Chang CS, and Karnik S (2000) Agonist-induced phosphorylation of the angiotensin II (AT $(1 \mathrm{~A})$ ) receptor requires generation of a conformation that is distinct from the inositol phosphate-signaling state. $J$ Biol Chem 275: $2893-2900$

Thway TM, Shlykov SG, Day MC, Sanborn BM, Gilstrap LC 3rd, Xia Y, and Kellems $\mathrm{RE}$ (2004) Antibodies from preeclamptic patients stimulate increased intracellular $\mathrm{Ca} 2+$ mobilization through angiotensin receptor activation. Circulation 110 1612-1619.

Tigerstedt R and Bergman PQ (1898) Niere und Kreislauf. Skand Arch Physiol 8: $223-271$.

Tirupula KC, Desnoyer R, Speth RC, and Karnik SS (2014) Atypical signaling and functional desensitization response of MAS receptor to peptide ligands. PLoS One 9:e103520.

Toda N, Ayajiki K, and Okamura T (2007) Interaction of endothelial nitric oxide and angiotensin in the circulation. Pharmacol Rev 59:54-87.

Tohgo A, Choy EW, Gesty-Palmer D, Pierce KL, Laporte S, Oakley RH, Caron MG, Lefkowitz RJ, and Luttrell LM (2003) The stability of the G protein-coupled receptor-beta-arrestin interaction determines the mechanism and functional consequence of ERK activation. J Biol Chem 278:6258-6267.

Tohgo A, Pierce KL, Choy EW, Lefkowitz RJ, and Luttrell LM (2002) beta-Arrestin scaffolding of the ERK cascade enhances cytosolic ERK activity but inhibits ERKmediated transcription following angiotensin AT1a receptor stimulation. $J$ Biol Chem 277:9429-9436.

Torsoni MA, Carvalheira JB, Calegari VC, Bezerra RM, Saad MJ, Gontijo JA and Velloso LA (2004) Angiotensin II (AngII) induces the expression of suppressor of cytokine signaling (SOCS)-3 in rat hypothalamus - a mechanism for desensitization of AngII signaling. J Endocrinol 181:117-128. 
Touyz RM (2004) Reactive oxygen species and angiotensin II signaling in vascular cells - implications in cardiovascular disease. Braz J Med Biol Res 37:1263-1273.

Touyz RM, He G, El Mabrouk M, and Schiffrin EL (2001a) p38 Map kinase regulates vascular smooth muscle cell collagen synthesis by angiotensin II in SHR but not in WKY. Hypertension 37:574-580.

Touyz RM, He G, Wu XH, Park JB, Mabrouk ME, and Schiffrin EL (2001b) Src is an important mediator of extracellular signal-regulated kinase 1/2-dependent growth signaling by angiotensin II in smooth muscle cells from resistance arteries of hypertensive patients. Hypertension 38:56-64.

Touyz RM, Yao G, Quinn MT, Pagano PJ, and Schiffrin EL (2005) p47phox associates with the cytoskeleton through cortactin in human vascular smooth muscle cells: role in NAD $(\mathrm{P}) \mathrm{H}$ oxidase regulation by angiotensin II. Arterioscler Thromb Vasc Biol 25:512-518.

Tsujimoto M and Hattori A (2005) The oxytocinase subfamily of M1 aminopeptidases. Biochim Biophys Acta 1751:9-18.

Tsutamoto T, Wada A, Maeda K, Mabuchi N, Hayashi M, Tsutsui T, Ohnishi M, Sawaki M, Fujii M, and Matsumoto T, et al. (2000) Angiotensin II type 1 receptor antagonist decreases plasma levels of tumor necrosis factor alpha, interleukin-6 and soluble adhesion molecules in patients with chronic heart failure. $J$ Am Coll Cardiol 35:714-721.

Tsutsumi K and Saavedra JM (1991) Differential development of angiotensin II receptor subtypes in the rat brain. Endocrinology 128:630-632.

Tsutsumi Y, Matsubara H, Masaki H, Kurihara H, Murasawa S, Takai S, Miyazaki M, Nozawa Y, Ozono R, and Nakagawa K, et al. (1999) Angiotensin II type 2 receptor overexpression activates the vascular kinin system and causes vasodilation. J Clin Invest 104:925-935

Tsutsumi Y, Matsubara H, Ohkubo N, Mori Y, Nozawa Y, Murasawa S, Kijima K Maruyama K, Masaki H, and Moriguchi Y, et al. (1998) Angiotensin II type 2 receptor is upregulated in human heart with interstitial fibrosis, and cardiac fibroblasts are the major cell type for its expression. Circ Res 83:1035-1046.

Tsuzuki S, Eguchi S, and Inagami T (1996a) Inhibition of cell proliferation and activation of protein tyrosine phosphatase mediated by angiotensin II type 2 (AT2) receptor in R3T3 cells. Biochem Biophys Res Commun 228:825-830.

Tsuzuki S, Matoba T, Eguchi S, and Inagami T (1996b) Angiotensin II type 2 receptor inhibits cell proliferation and activates tyrosine phosphatase. Hypertension 28 916-918

Tu ML, Wang HQ, Lei HD, Luo GS, Liu XJ, Liu WS, Xiong C, Liu YQ, and Ren SQ (2005) [Effects of adenoviral vector containing human angiotensin II type 1 receptor antisense cDNA on biological action of human pulmonary artery smooth muscle cells]. Zhonghua Jie He He Hu Xi Za Zhi. 28:263-267.

Turner NA, Ball SG, and Balmforth AJ (2001) The mechanism of angiotensin II-induced extracellular signal-regulated kinase-1/2 activation is independent of angiotensin AT(1A) receptor internalisation. Cell Signal 13:269-277.

Turu G, Szidonya L, Gáborik Z, Buday L, Spät A, Clark AJ, and Hunyady L (2006) Differential beta-arrestin binding of AT1 and AT2 angiotensin receptors. FEBS Lett 580:41-45.

Uchiyama-Tanaka Y, Matsubara H, Nozawa Y, Murasawa S, Mori Y, Kosaki A, Maruyama K, Masaki H, Shibasaki Y, and Fujiyama S, et al. (2001) Angiotensin II signaling and HB-EGF shedding via metalloproteinase in glomerular mesangial cells. Kidney Int 60:2153-2163.

Ueki M, Koda M, Shimizu T, Mitsuta A, Yamamoto T, and Murawaki Y (2009) Effect of an angiotensin-II type-1 receptor blocker, candesartan on hepatic fibrosis in chronic hepatitis C: a prospective study. Hepatogastroenterology 56:1100-1104.

Ueki M, Koda M, Yamamoto S, Matsunaga Y, and Murawaki Y (2006) Preventive and therapeutic effects of angiotensin II type 1 receptor blocker on hepatic fibrosis induced by bile duct ligation in rats. $J$ Gastroenterol 41:996-1004.

Uemura H, Hoshino K, and Kubota Y (2011) Engagement of renin-angiotensin system in prostate cancer. Curr Cancer Drug Targets 11:442-450.

Uemura H, Ishiguro H, and Kubota Y (2006) Angiotensin II receptor blocker: possibility of antitumor agent for prostate cancer. Mini Rev Med Chem 6:835-844.

Uemura H and Kubota Y (2012) [Cancer and renin-angiotensin system]. Nihon Rinsho 70:1530-1535.

Uemura H, Nakaigawa N, Ishiguro H, and Kubota Y (2005) Antiproliferative efficacy of angiotensin II receptor blockers in prostate cancer. Curr Cancer Drug Targets 5: 307-323.

Ulmasov B, Xu Z, Tetri LH, Inagami T, and Neuschwander-Tetri BA (2009) Protective role of angiotensin II type 2 receptor signaling in a mouse model of pancreatic fibrosis. Am J Physiol Gastrointest Liver Physiol 296:G284-G294.

Unal H, Jagannathan R, Bhat MB, and Karnik SS (2010) Ligand-specific conformation of extracellular loop-2 in the angiotensin II type 1 receptor. J Biol Chem 285:16341-16350

Unal H, Jagannathan R, Bhatnagar A, Tirupula K, Desnoyer R, and Karnik SS (2013) Long range effect of mutations on specific conformational changes in the extracellular loop 2 of angiotensin II type 1 receptor. J Biol Chem 288:540-551.

Unal H, Jagannathan R, and Karnik SS (2012) Mechanism of GPCR-directed autoantibodies in diseases. Adv Exp Med Biol 749:187-199.

Unal H and Karnik SS (2012) Domain coupling in GPCRs: the engine for induced conformational changes. Trends Pharmacol Sci 33:79-88.

Unal $\mathrm{H}$ and Karnik SS (2014) Constitutive activity in the angiotensin II type 1 receptor: discovery and applications. Adv Pharmacol 70:155-174.

Unger T (1999) The angiotensin type 2 receptor: variations on an enigmatic theme. $J$ Hypertens 17:1775-1786.

Urban JD, Clarke WP, von Zastrow M, Nichols DE, Kobilka B, Weinstein H, Javitch JA, Roth BL, Christopoulos A, and Sexton PM, et al. (2007) Functional selectivity and classical concepts of quantitative pharmacology. J Pharmacol Exp Ther 320: $1-13$.

Ushio-Fukai M, Alexander RW, Akers M, Lyons PR, Lassègue B, and Griendling KK (1999a) Angiotensin II receptor coupling to phospholipase D is mediated by the betagamma subunits of heterotrimeric $\mathrm{G}$ proteins in vascular smooth muscle cells. Mol Pharmacol 55:142-149.
Ushio-Fukai M, Alexander RW, Akers M, Yin Q, Fujio Y, Walsh K, and Griendling KK (1999b) Reactive oxygen species mediate the activation of Akt/protein kinase B by angiotensin II in vascular smooth muscle cells. J Biol Chem 274: 22699-22704.

Ushio-Fukai M, Zafari AM, Fukui T, Ishizaka N, and Griendling KK (1996) p22phox is a critical component of the superoxide-generating NADH/NADPH oxidase system and regulates angiotensin II-induced hypertrophy in vascular smooth muscle cells. J Biol Chem 271:23317-23321.

Vallega GA, Canessa ML, Berk BC, Brock TA, and Alexander RW (1988) Vascular smooth muscle $\mathrm{Na}+\mathrm{H}+$ exchanger kinetics and its activation by angiotensin II. Am J Physiol 254:C751-C758.

Vanderheyden PM, Fierens FL, De Backer J, and Vauquelin G (2000a) Reversible and syntopic interaction between angiotensin receptor antagonists on Chinese hamster ovary cells expressing human angiotensin II type 1 receptors. Biochem Pharmacol 59:927-935.

Vanderheyden PM, Fierens FL, and Vauquelin G (2000b) Angiotensin II type 1 receptor antagonists. Why do some of them produce insurmountable inhibition? Biochem Pharmacol 60:1557-1563.

van Geel PP, Pinto YM, Voors AA, Buikema H, Oosterga M, Crijns HJ, and van Gilst WH (2000) Angiotensin II type 1 receptor A1166C gene polymorphism is associated with an increased response to angiotensin II in human arteries. Hypertension 35: $717-721$.

van Kesteren CA, van Heugten HA, Lamers JM, Saxena PR, Schalekamp MA, and Danser AH (1997) Angiotensin II-mediated growth and antigrowth effects in cultured neonatal rat cardiac myocytes and fibroblasts. J Mol Cell Cardiol 29: $2147-2157$.

Van Liefde I and Vauquelin G (2009) Sartan-AT1 receptor interactions: in vitro evidence for insurmountable antagonism and inverse agonism. Mol Cell Endocrinol 302:237-243.

van't Veer LJ, van der Feltz MJ, van den Berg-Bakker CA, Cheng NC, Hermens RP, van Oorschot DA, Kievits T, and Schrier PI (1993) Activation of the mas oncogene involves coupling to human alphoid sequences. Oncogene 8:2673-2681.

Vauquelin G, Michotte Y, Smolders I, Sarre S, Ebinger G, Dupont A, and Vanderheyden P (2002a) Cellular targets for angiotensin II fragments: pharmacological and molecular evidence. J Renin Angiotensin Aldosterone Syst $\mathbf{3}$ : 195-204

Vauquelin G, Van Liefde I, Birzbier BB, and Vanderheyden PM (2002b) New insights in insurmountable antagonism. Fundam Clin Pharmacol 16:263-272.

Velloso EP, Vieira R, Cabral AC, Kalapothakis E, and Santos RA (2007) Reduced plasma levels of angiotensin-(1-7) and renin activity in preeclamptic patients are associated with the angiotensin I- converting enzyme deletion/deletion genotype. Braz J Med Biol Res 40:583-590.

Venema RC, Ju H, Venema VJ, Schieffer B, Harp JB, Ling BN, Eaton DC and Marrero MB (1998a) Angiotensin II-induced association of phospholipase Cgamma1 with the G-protein-coupled AT1 receptor. J Biol Chem 273:7703-7708.

Venema RC, Venema VJ, Eaton DC, and Marrero MB (1998b) Angiotensin II-induced tyrosine phosphorylation of signal transducers and activators of transcription 1 is regulated by Janus-activated kinase 2 and Fyn kinases and mitogen-activated protein kinase phosphatase 1. J Biol Chem 273:30795-30800.

Verano-Braga T, Schwammle V, Sylvester M, Passos-Silva DG, Peluso AA, Etelvino GM, Santos RA, and Roepstorff P (2012) Time-resolved quantitative phosphoproteomics: new insights into angiotensin-(1-7) signaling networks in human endothelial cells. J Proteome Res 11:3370-3381.

Verdonk K, Durik M, Abd-Alla N, Batenburg WW, van den Bogaerdt AJ, van Veghel R, Roks AJ, Danser AH, and van Esch JH (2012) Compound 21 induces vasorelaxation via an endothelium- and angiotensin II type 2 receptor-independent mechanism. Hypertension 60:722-729.

Verheijen I, De Backer JP, Vanderheyden P, and Vauquelin G (2003) A two-state model of antagonist-AT1 receptor interaction: further support by binding studies at low temperature. Biochem Pharmacol 65:1339-1341.

Verheijen I, Fierens FL, Debacker JP, Vauquelin G, and Vanderheyden PM (2000) Interaction between the partially insurmountable antagonist valsartan and human recombinant angiotensin II type 1 receptors. Fundam Clin Pharmacol 14:577-585.

Verlander JW, Hong S, Pech V, Bailey JL, Agazatian D, Matthews SW, Coffman TM, Le T, Inagami T, and Whitehill FM, et al. (2011) Angiotensin II acts through the angiotensin 1a receptor to upregulate pendrin. Am J Physiol Renal Physiol 301: F1314-F1325.

Vervoort VS, Beachem MA, Edwards PS, Ladd S, Miller KE, de Mollerat X, Clarkson K, DuPont B, Schwartz CE, and Stevenson RE, et al. (2002) AGTR2 mutations in $\mathrm{X}$-linked mental retardation. Science 296:2401-2403.

Viana GE, Pereira VM, Honorato-Sampaio K, Oliveira CA, Santos RA, and Reis AM (2011) Angiotensin-(1-7) induces ovulation and steroidogenesis in perfused rabbit ovaries. Exp Physiol 96:957-965.

Villar AJ and Pedersen RA (1994) Parental imprinting of the Mas protooncogene in mouse. Nat Genet 8:373-379.

Vinh A, Widdop RE, Drummond GR, and Gaspari TA (2008) Chronic angiotensin IV treatment reverses endothelial dysfunction in ApoE-deficient mice. Cardiovasc Res 77:178-187.

Violin JD, DeWire SM, Yamashita D, Rominger DH, Nguyen L, Schiller K, Whalen EJ, Gowen M, and Lark MW (2010) Selectively engaging $\beta$-arrestins at the angiotensin II type 1 receptor reduces blood pressure and increases cardiac performance. $J$ Pharmacol Exp Ther 335:572-579.

Violin JD and Lefkowitz RJ (2007) Beta-arrestin-biased ligands at seventransmembrane receptors. Trends Pharmacol Sci 28:416-422.

Violin JD, Soergel DG, Boerrigter G, Burnett JC Jr, and Lark MW (2013) GPCR biased ligands as novel heart failure therapeutics. Trends Cardiovasc Med 23: 242-249.

Viswanathan M and Saavedra JM (1992) Expression of angiotensin II AT2 receptors in the rat skin during experimental wound healing. Peptides 13:783-786. 
Von Bohlen und Halbach O, Walther T, Bader M, and Albrecht D (2000) Interaction between Mas and the angiotensin AT1 receptor in the amygdala. $J$ Neurophysiol 83:2012-2021.

von Bohlen und Halbach O, Walther T, Bader M, and Albrecht D (2001) Genetic deletion of angiotensin AT2 receptor leads to increased cell numbers in different brain structures of mice. Regul Pept 99:209-216.

Vyas VK and Ghate M (2010) Substituted benzimidazole derivatives as angiotensin II-AT1 receptor antagonist: a review. Mini Rev Med Chem 10:1366-1384.

Wagh PK, Peace BE, and Waltz SE (2008) Met-related receptor tyrosine kinase Ron in tumor growth and metastasis. Adv Cancer Res 100:1-33.

Wakui H and Tamura K (2012) [Physiology of novel AT1 receptor-binding molecule, ATRAP]. Nihon Rinsho 70:1499-1503.

Wallis MG, Lankford MF, and Keller SR (2007) Vasopressin is a physiological substrate for the insulin-regulated aminopeptidase IRAP. Am J Physiol Endocrinol Metab 293:E1092-E1102.

Wallukat G, Homuth V, Fischer T, Lindschau C, Horstkamp B, Jüpner A, Baur E, Nissen E, Vetter K, and Neichel D, et al. (1999) Patients with preeclampsia develop agonistic autoantibodies against the angiotensin AT1 receptor. $J$ Clin Invest 103: 945-952.

Walters PE, Gaspari TA, and Widdop RE (2005) Angiotensin-(1-7) acts as a vasodepressor agent via angiotensin II type 2 receptors in conscious rats. Hypertension 45:960-966.

Walther T, Balschun D, Voigt JP, Fink H, Zuschratter W, Birchmeier C, Ganten D, and Bader M (1998) Sustained long term potentiation and anxiety in mice lacking the Mas protooncogene. J Biol Chem 273:11867-11873.

Walther T, Voigt JP, Fink H, and Bader M (2000a) Sex specific behavioural alterations in Mas-deficient mice. Behav Brain Res 107:105-109.

Walther T, Wessel N, Kang N, Sander A, Tschöpe C, Malberg H, Bader M, and Voss A (2000b) Altered heart rate and blood pressure variability in mice lacking the Mas protooncogene. Braz J Med Biol Res 33:1-9.

Wan Y, Wallinder C, Plouffe B, Beaudry H, Mahalingam AK, Wu X, Johansson B, Holm M, Botoros M, and Karlén A, et al. (2004) Design, synthesis, and biological evaluation of the first selective nonpeptide AT2 receptor agonist. J Med Chem 47: $5995-6008$

Wang D, Yu X, Cohen RA, and Brecher P (2000a) Distinct effects of N-acetylcysteine and nitric oxide on angiotensin II-induced epidermal growth factor receptor phosphorylation and intracellular $\mathrm{Ca}(2+)$ levels. J Biol Chem 275:12223-12230.

Wang H, Lu D, Reaves PY, Katovich MJ, and Raizada MK (2000b) Retrovirally mediated delivery of angiotensin II type 1 receptor antisense in vitro and in vivo. Methods Enzymol 314:581-590.

Wang J, Paradis P, Aries A, Komati H, Lefebvre C, Wang H, and Nemer M (2005) Convergence of protein kinase $\mathrm{C}$ and JAK-STAT signaling on transcription factor GATA-4. Mol Cell Biol 25:9829-9844.

Warnecke C, Kaup D, Marienfeld U, Poller W, Yankah C, Gräfe M, Fleck E, and Regitz-Zagrosek V (2001) Adenovirus-mediated overexpression and stimulation of the human angiotensin II type 2 receptor in porcine cardiac fibroblasts does not modulate proliferation, collagen I mRNA expression and ERK1/ERK2 activity, but inhibits protein tyrosine phosphatases. $J$ Mol Med (Berl) 79:510-521.

Warnecke C, Sürder D, Curth R, Fleck E, and Regitz-Zagrosek V (1999) Analysis and functional characterization of alternatively spliced angiotensin II type 1 and 2 receptor transcripts in the human heart. J Mol Med (Berl) 77:718-727.

Wasse H, Naqvi N, and Husain A (2012) Impact of Mast Cell Chymase on Renal Disease Progression. Curr Hypertens Rev 8:15-23.

Wassmann S, Czech T, van Eickels M, Fleming I, Böhm M, and Nickenig G (2004a) Inhibition of diet-induced atherosclerosis and endothelial dysfunction in apolipoprotein E/angiotensin II type 1A receptor double-knockout mice. Circulation 110 3062-3067.

Wassmann S, Stumpf M, Strehlow K, Schmid A, Schieffer B, Böhm M, and Nickenig G (2004b) Interleukin-6 induces oxidative stress and endothelial dysfunction by overexpression of the angiotensin II type 1 receptor. Circ Res 94:534-541.

Wayner MJ, Armstrong DL, Phelix CF, Wright JW, and Harding JW (2001) Angiotensin IV enhances LTP in rat dentate gyrus in vivo. Peptides 22:1403-1414.

Webb ML, Liu EC, Cohen RB, Hedberg A, Bogosian EA, Monshizadegan H, Molloy C, Serafino R, Moreland S, and Murphy TJ, et al. (1992) Molecular characterization of angiotensin II type II receptors in rat pheochromocytoma cells. Peptides 13:499-508.

Weber MA, Byyny RL, Pratt JH, Faison EP, Snavely DB, Goldberg AI, and Nelson EB (1995) Blood pressure effects of the angiotensin II receptor blocker, losartan. Arch Intern Med 155:405-411.

Wei H, Ahn S, Barnes WG, and Lefkowitz RJ (2004) Stable interaction between beta-arrestin 2 and angiotensin type $1 \mathrm{~A}$ receptor is required for beta-arrestin 2-mediated activation of extracellular signal-regulated kinases 1 and 2 . J Biol Chem 279:48255-48261.

Wei H, Ahn S, Shenoy SK, Karnik SS, Hunyady L, Luttrell LM, and Lefkowitz RJ (2003) Independent beta-arrestin 2 and G protein-mediated pathways for angiotensin II activation of extracellular signal-regulated kinases 1 and 2. Proc Nat Acad Sci USA 100:10782-10787.

Weidekamm C, Hauser P, Hansmann C, Schwarz C, Klingler H, Mayer G, and Oberbauer R (2002) Effects of AT1 and AT2 receptor blockade on angiotensin II induced apoptosis of human renal proximal tubular epithelial cells. Wien Klin Wochenschr 114:725-729.

Weiss D, Kools JJ, and Taylor WR (2001) Angiotensin II-induced hypertension accelerates the development of atherosclerosis in apoE-deficient mice. Circulation 103:448-454

Wharton J, Morgan K, Rutherford RA, Catravas JD, Chester A, Whitehead BF, De Leval MR, Yacoub MH, and Polak JM (1998) Differential distribution of angiotensin AT2 receptors in the normal and failing human heart. $J$ Pharmacol Exp Ther 284:323-336.

Whitebread S, Mele M, Kamber B, and de Gasparo M (1989) Preliminary biochemical characterization of two angiotensin II receptor subtypes. Biochem Biophys Res Commun 163:284-291.
Whitehead IP, Zohn IE, and Der CJ (2001) Rho GTPase-dependent transformation by G protein-coupled receptors. Oncogene 20:1547-1555.

Widdop RE, Jones ES, Hannan RE, and Gaspari TA (2003) Angiotensin AT2 receptors: cardiovascular hope or hype? Br J Pharmacol 140:809-824.

Wiemer G, Dobrucki LW, Louka FR, Malinski T, and Heitsch H (2002) AVE 0991, a nonpeptide mimic of the effects of angiotensin-(1-7) on the endothelium. Hypertension 40:847-852.

Wierzbicki AS, Lambert-Hammill M, Lumb PJ, and Crook MA (2000) Reninangiotensin system polymorphisms and coronary events in familial hypercholesterolemia. Hypertension 36:808-812.

Wiest SA, Rampersaud A, Zimmerman K, and Steinberg MI (1991) Characterization of distinct angiotensin II binding sites in rat adrenal gland and bovine cerebellum using selective nonpeptide antagonists. J Cardiovasc Pharmacol 17:177-184.

Wilson BA, Cruz-Diaz N, Marshall AC, Pirro NT, Su Y, Gwathmey TM, Rose JC, and Chappell MC (2015) An angiotensin-(1-7) peptidase in the kidney cortex, proximal tubules, and human HK-2 epithelial cells that is distinct from insulindegrading enzyme. Am J Physiol Renal Physiol 308:F594-F601.

Wilson PC, Lee MH, Appleton KM, El-Shewy HM, Morinelli TA, Peterson YK, Luttrell LM, and Jaffa AA (2013) The arrestin-selective angiotensin AT1 receptor agonist [Sar1,Ile4,Ile8]-AngII negatively regulates bradykinin B2 receptor signaling via AT1-B2 receptor heterodimers. J Biol Chem 288:18872-18884.

Wislez M, Bazot M, Milleron B, Bazelly B, Antoine M, Bigot JM, and Carette MF (1998) [Cystic opacity of the posterior mediastinum]. Rev Pneumol Clin 54:30-33.

Wolf G, Wenzel U, Burns KD, Harris RC, Stahl RA, and Thaiss F (2002) Angiotensin II activates nuclear transcription factor-kappaB through AT1 and AT2 receptors. Kidney Int 61:1986-1995

Woolfolk EA, Eguchi S, Ohtsu H, Nakashima H, Ueno H, Gerthoffer WT, and Motley ED (2005) Angiotensin II-induced activation of p21-activated kinase 1 requires $\mathrm{Ca} 2$ + and protein kinase Cdelta in vascular smooth muscle cells. Am J Physiol Cell Physiol 289:C1286-C1294.

Wright JW, Clemens JA, Panetta JA, Smalstig EB, Weatherly LA, Kramár EA Pederson ES, Mungall BH, and Harding JW (1996) Effects of LY231617 and angiotensin IV on ischemia-induced deficits in circular water maze and passive avoidance performance in rats. Brain Res 717:1-11.

Wright JW and Harding JW (1995) Brain angiotensin receptor subtypes AT1, AT2, and AT4 and their functions. Regul Pept 59:269-295.

Wright JW, Krebs LT, Stobb JW, and Harding JW (1995) The angiotensin IV system: functional implications. Front Neuroendocrinol 16:23-52.

Wright JW, Miller-Wing AV, Shaffer MJ, Higginson C, Wright DE, Hanesworth JM, and Harding JW (1993) Angiotensin II(3-8) (ANG IV) hippocampal binding: potential role in the facilitation of memory. Brain Res Bull 32:497-502.

Wright JW, Yamamoto BJ, and Harding JW (2008) Angiotensin receptor subtype mediated physiologies and behaviors: new discoveries and clinical targets. Prog Neurobiol 84:157-181.

Wruck CJ, Funke-Kaiser H, Pufe T, Kusserow H, Menk M, Schefe JH, Kruse ML Stoll M, and Unger T (2005) Regulation of transport of the angiotensin AT2 receptor by a novel membrane-associated Golgi protein. Arterioscler Thromb Vasc Biol 25:57-64.

Wu G, Zhao G, and He Y (2003) Distinct pathways for the trafficking of angiotensin II and adrenergic receptors from the endoplasmic reticulum to the cell surface: Rab1independent transport of a $\mathrm{G}$ protein-coupled receptor. J Biol Chem 278: $47062-47069$.

Wu KK (2002) Regulation of endothelial nitric oxide synthase activity and gene expression. Ann N Y Acad Sci 962:122-130.

Wu L, Iwai M, Nakagami H, Chen R, Suzuki J, Akishita M, de Gasparo M, and Horiuchi M (2002) Effect of angiotensin II type 1 receptor blockade on cardiac remodeling in angiotensin II type 2 receptor null mice. Arterioscler Thromb Vasc Biol 22:49-54.

Wu S, Gao J, Ohlemeyer C, Roos D, Niessen H, Köttgen E, and Gessner R (2005) Activation of AP-1 through reactive oxygen species by angiotensin II in rat cardiomyocytes. Free Radic Biol Med 39:1601-1610.

Xia Y and Kellems RE (2013) Angiotensin receptor agonistic autoantibodies and hypertension: preeclampsia and beyond. Circ Res 113:78-87.

Xia Y, Wen HY, and Kellems RE (2002) Angiotensin II inhibits human trophoblas invasion through AT1 receptor activation. J Biol Chem 277:24601-24608.

Xiao K, McClatchy DB, Shukla AK, Zhao Y, Chen M, Shenoy SK, Yates JR 3rd, and Lefkowitz RJ (2007) Functional specialization of beta-arrestin interactions revealed by proteomic analysis. Proc Natl Acad Sci USA 104:12011-12016.

Xiao K, Sun J, Kim J, Rajagopal S, Zhai B, Villén J, Haas W, Kovacs JJ, Shukla AK, and Hara MR, et al. (2010) Global phosphorylation analysis of beta-arrestinmediated signaling downstream of a seven transmembrane receptor (7TMR). Proc Natl Acad Sci USA 107:15299-15304

Xu P, Costa-Goncalves AC, Todiras M, Rabelo LA, Sampaio WO, Moura MM, Santos SS, Luft FC, Bader M, and Gross V, et al. (2008) Endothelial dysfunction and elevated blood pressure in MAS gene-deleted mice. Hypertension 51:574-580.

$\mathrm{Xu}$ P, Santos RA, Bader M, and Alenina N (2007) Alterations in gene expression in the testis of angiotensin-(1-7)-receptor Mas-deficient mice. Regul Pept 138:51-55.

Yahata Y, Shirakata Y, Tokumaru S, Yang L, Dai X, Tohyama M, Tsuda T, Sayama K, Iwai M, and Horiuchi M, et al. (2006) A novel function of angiotensin II in skin wound healing. Induction of fibroblast and keratinocyte migration by angiotensin II via heparin-binding epidermal growth factor (EGF)-like growth factor-mediated EGF receptor transactivation. J Biol Chem 281:13209-13216.

Yamada H, Akishita M, Ito M, Tamura K, Daviet L, Lehtonen JY, Dzau VJ, and Horiuchi M (1999) AT2 receptor and vascular smooth muscle cell differentiation in vascular development. Hypertension 33:1414-1419.

Yamada M, Kushibiki M, Osanai T, Tomita H, and Okumura K (2008) Vasoconstrictor effect of aldosterone via angiotensin II type 1 (AT1) receptor: possible role of AT1 receptor dimerization. Cardiovasc Res 79:169-178.

Yamada T, Akishita M, Pollman MJ, Gibbons GH, Dzau VJ, and Horiuchi M (1998) Angiotensin II type 2 receptor mediates vascular smooth muscle cell apoptosis and 
antagonizes angiotensin II type 1 receptor action: an in vitro gene transfer study. Life Sci 63:PL289-PL295.

Yamada T, Horiuchi M, and Dzau VJ (1996) Angiotensin II type 2 receptor mediates programmed cell death. Proc Natl Acad Sci USA 93:156-160.

Yamano Y, Ohyama K, Chaki S, Guo DF, and Inagami T (1992) Identification of amino acid residues of rat angiotensin II receptor for ligand binding by site directed mutagenesis. Biochem Biophys Res Commun 187:1426-1431.

Yamano Y, Ohyama K, Kikyo M, Sano T, Nakagomi Y, Inoue Y, Nakamura N, Morishima I, Guo DF, and Hamakubo T, et al. (1995) Mutagenesis and the molecular modeling of the rat angiotensin II receptor (AT1). J Biol Chem 270: 14024-14030.

Yamazaki T, Komuro I, Kudoh S, Zou Y, Shiojima I, Mizuno T, Takano H, Hiroi Y, Ueki K, and Tobe K, et al. (1995) Angiotensin II partly mediates mechanical stressinduced cardiac hypertrophy. Circ Res 77:258-265.

Yan C, Kim D, Aizawa T, and Berk BC (2003a) Functional interplay between angiotensin II and nitric oxide: cyclic GMP as a key mediator. Arterioscler Thromb Vasc Biol 23:26-36.

Yan L, Holleran BJ, Lavigne P, Escher E, Guillemette G, and Leduc R (2010) Analysis of transmembrane domains 1 and 4 of the human angiotensin II AT1 receptor by cysteine-scanning mutagenesis. J Biol Chem 285:2284-2293.

Yan X, Price RL, Nakayama M, Ito K, Schuldt AJ, Manning WJ, Sanbe A, Borg TK Robbins J, and Lorell BH (2003b) Ventricular-specific expression of angiotensin II type 2 receptors causes dilated cardiomyopathy and heart failure in transgenic mice. Am J Physiol Heart Circ Physiol 285:H2179-H2187.

Yang BC, Phillips MI, Mohuczy D, Meng H, Shen L, Mehta P, and Mehta JL (1998) Increased angiotensin II type 1 receptor expression in hypercholesterolemic atherosclerosis in rabbits. Arterioscler Thromb Vasc Biol 18:1433-1439.

Yang R, Smolders I, De Bundel D, Fouyn R, Halberg M, Demaegdt H, Vanderheyden $\mathrm{P}$, and Dupont AG (2008) Brain and peripheral angiotensin II type 1 receptors mediate renal vasoconstrictor and blood pressure responses to angiotensin IV in the rat. J Hypertens 26:998-1007.

Yang R, Walther T, Gembardt F, Smolders I, Vanderheyden P, Albiston AL, Chai SY, and Dupont AG (2010) Renal vasoconstrictor and pressor responses to angiotensin IV in mice are AT1a-receptor mediated. J Hypertens 28:487-494.

Yang X, Taylor L, and Polgar P (1999) Effect of the G-protein, G alpha(i2), and G alpha(i3) subunit knockdown on bradykinin-induced signal transduction in rat-1 cells. Mol Cell Biol Res Commun 1:227-236.

Yasuda N, Akazawa H, Ito K, Shimizu I, Kudo-Sakamoto Y, Yabumoto C, Yano M, Yamamoto R, Ozasa Y, and Minamino T, et al. (2012) Agonist-independent constitutive activity of angiotensin II receptor promotes cardiac remodeling in mice. Hypertension 59:627-633.

Yasuda N, Akazawa H, Qin Y, Zou Y, and Komuro I (2008a) A novel mechanism of mechanical stress-induced angiotensin II type 1-receptor activation without the involvement of angiotensin II. Naunyn Schmiedebergs Arch Pharmacol 377: 393-399.

Yasuda N, Miura S, Akazawa H, Tanaka T, Qin Y, Kiya Y, Imaizumi S, Fujino M, Ito $\mathrm{K}$, and Zou Y, et al. (2008b) Conformational switch of angiotensin II type 1 receptor underlying mechanical stress-induced activation. EMBO Rep 9:179-186.

Yayama K, Hiyoshi H, Imazu D, and Okamoto H (2006) Angiotensin II stimulates endothelial NO synthase phosphorylation in thoracic aorta of mice with abdominal aortic banding via type 2 receptor. Hypertension 48:958-964.

Ye S, Chai SY, Lew RA, and Albiston AL (2007) Insulin-regulated aminopeptidase: analysis of peptide substrate and inhibitor binding to the catalytic domain. Biol Chem 388:399-403

Ye S, Chai SY, Lew RA, Ascher DB, Morton CJ, Parker MW, and Albiston AL (2008) Identification of modulating residues defining the catalytic cleft of insulinregulated aminopeptidase. Biochem Cell Biol 86:251-261.

Yee DK, Suzuki A, Luo L, and Fluharty SJ (2006) Identification of structural determinants for $\mathrm{G}$ protein-independent activation of mitogen-activated protein kinases in the seventh transmembrane domain of the angiotensin II type 1 receptor. Mol Endocrinol 20:1924-1934.

Yoshiji H, Noguchi R, Ikenaka Y, Namisaki T, Kitade M, Kaji K, Shirai Y, Yoshii J, Yanase K, and Yamazaki M, et al. (2009) Losartan, an angiotensin-II type 1 receptor blocker, attenuates the liver fibrosis development of non-alcoholic steatohepatitis in the rat. BMC Res Notes 2:70.

You D, Loufrani L, Baron C, Levy BI, Widdop RE, and Henrion D (2005) High blood pressure reduction reverses angiotensin II type 2 receptor-mediated vasoconstriction into vasodilation in spontaneously hypertensive rats. Circulation 111 1006-1011.

Young D, O'Neill K, Jessell T, and Wigler M (1988) Characterization of the rat mas oncogene and its high-level expression in the hippocampus and cerebral cortex of rat brain. Proc Natl Acad Sci USA 85:5339-5342.

Young D, Waitches G, Birchmeier C, Fasano O, and Wigler M (1986) Isolation and characterization of a new cellular oncogene encoding a protein with multiple potential transmembrane domains. Cell 45:711-719.

Yue H, Li W, Desnoyer R, and Karnik SS (2010) Role of nuclear unphosphorylated STAT3 in angiotensin II type 1 receptor-induced cardiac hypertrophy. Cardiovasc Res 85:90-99.

Yusuf S, Sleight P, Pogue J, Bosch J, Davies R, and Dagenais G; The Heart Outcomes Prevention Evaluation Study Investigators (2000) Effects of an angiotensinconverting-enzyme inhibitor, ramipril, on cardiovascular events in high-risk patients. $N$ Engl J Med 342:145-153.

Zafari AM, Ushio-Fukai M, Akers M, Yin Q, Shah A, Harrison DG, Taylor WR, and Griendling KK (1998) Role of NADH/NADPH oxidase-derived H2O2 in angiotensin II-induced vascular hypertrophy. Hypertension 32:488-495.

Zaman MA, Oparil S, and Calhoun DA (2002) Drugs targeting the renin-angiotensinaldosterone system. Nat Rev Drug Discov 1:621-636.

Zeng C, Asico LD, Wang X, Hopfer U, Eisner GM, Felder RA, and Jose PA (2003a) Angiotensin II regulation of AT1 and D3 dopamine receptors in renal proximal tubule cells of SHR. Hypertension 41:724-729.
Zeng C, Liu Y, Wang Z, He D, Huang L, Yu P, Zheng S, Jones JE, Asico LD, and Hopfer U, et al. (2006) Activation of D3 dopamine receptor decreases angiotensin II type 1 receptor expression in rat renal proximal tubule cells. Circ Res $\mathbf{9 9}$ : 494-500.

Zeng C, Luo Y, Asico LD, Hopfer U, Eisner GM, Felder RA, and Jose PA (2003b) Perturbation of D1 dopamine and AT1 receptor interaction in spontaneously hypertensive rats. Hypertension 42:787-792.

Zeng C, Wang Z, Asico LD, Hopfer U, Eisner GM, Felder RA, and Jose PA (2005a) Aberrant ETB receptor regulation of AT receptors in immortalized renal proximal tubule cells of spontaneously hypertensive rats. Kidney Int 68:623-631.

Zeng C, Yang Z, Wang Z, Jones J, Wang X, Altea J, Mangrum AJ, Hopfer U, Sibley DR, and Eisner GM, et al. (2005b) Interaction of angiotensin II type 1 and D5 dopamine receptors in renal proximal tubule cells. Hypertension 45:804-810.

Zhai P, Galeotti J, Liu J, Holle E, Yu X, Wagner T, and Sadoshima J (2006) An angiotensin II type 1 receptor mutant lacking epidermal growth factor receptor ransactivation does not induce angiotensin II-mediated cardiac hypertrophy. Circ Res 99:528-536.

Zhai P, Yamamoto M, Galeotti J, Liu J, Masurekar M, Thaisz J, Irie K, Holle E, Yu X, and Kupershmidt S, et al. (2005) Cardiac-specific overexpression of AT1 receptor mutant lacking $\mathrm{G}$ alpha $\mathrm{q} / \mathrm{G}$ alpha i coupling causes hypertrophy and bradycardia in transgenic mice. J Clin Invest 115:3045-3056.

Zhang H, Unal H, Gati C, Han GW, Liu W, Zatsepin NA, James D, Wang D, Nelson $\mathrm{G}$, and Weierstall U, et al. (2015) Structure of the angiotensin receptor revealed by serial femtosecond crystallography. Cell 161:833-844.

Zhang GY, Li X, Yi CG, Pan H, He GD, Yu Q, Jiang LF, Xu WH, Li ZJ, and Ding J, et al. (2009) Angiotensin II activates connective tissue growth factor and induces extracellular matrix changes involving Smad/activation and p38 mitogen-activated protein kinase signalling pathways in human dermal fibroblasts. Exp Dermatol 18:947-953.

Zhang J and Pratt RE (1996) The AT2 receptor selectively associates with Gialpha2 and Gialpha3 in the rat fetus. J Biol Chem 271:15026-15033.

Zhang JH, Hanesworth JM, Sardinia MF, Alt JA, Wright JW, and Harding JW (1999) Structural analysis of angiotensin IV receptor (AT4) from selected bovine tissues. $J$ Pharmacol Exp Ther 289:1075-1083.

Zhang M, Zhao X, Chen HC, Catt KJ, and Hunyady L (2000) Activation of the AT1 angiotensin receptor is dependent on adjacent apolar residues in the carboxyl terminus of the third cytoplasmic loop. J Biol Chem 275:15782-15788.

Zhang Q, Thomas SM, Lui VW, Xi S, Siegfried JM, Fan H, Smithgall TE, Mills GB, and Grandis JR (2006) Phosphorylation of TNF-alpha converting enzyme by gastrin-releasing peptide induces amphiregulin release and EGF receptor activation. Proc Natl Acad Sci USA 103:6901-6906.

Zhang T, Li Z, Dang H, Chen R, Liaw C, Tran TA, Boatman PD, Connolly DT, and Adams JW (2012) Inhibition of Mas G-protein signaling improves coronary blood flow, reduces myocardial infarct size, and provides long-term cardioprotection. Am J Physiol Heart Circ Physiol 302:H299-H311.

Zhao X, Li X, Trusa S, and Olson SC (2005a) Angiotensin type 1 receptor is linked to inhibition of nitric oxide production in pulmonary endothelial cells. Regul Pept 132: $113-122$

Zhao Y, Chen X, Cai L, Yang Y, Sui G, and Fu S (2010) Angiotensin II/angiotensin II type I receptor (AT1R) signaling promotes MCF-7 breast cancer cells survival via PI3-kinase/Akt pathway. J Cell Physiol 225:168-173.

Zhao Y, Foryst-Ludwig A, Bruemmer D, Culman J, Bader M, Unger T, and Kintscher U (2005b) Angiotensin II induces peroxisome proliferator-activated receptor gamma in PC12W cells via angiotensin type 2 receptor activation. $J$ Neurochem 94:1395-1401. Zhou A, Dekker GA, Lumbers ER, Lee SY, Thompson SD, McCowan LM, and Roberts CT; SCOPE consortium (2013) The association of AGTR2 polymorphisms with preeclampsia and uterine artery bilateral notching is modulated by maternal BMI. Placenta 34:75-81.

Zhou CC, Ahmad S, Mi T, Abbasi S, Xia L, Day MC, Ramin SM, Ahmed A, Kellems RE, and Xia Y (2008a) Autoantibody from women with preeclampsia induces soluble Fmslike tyrosine kinase-1 production via angiotensin type 1 receptor and calcineurin nuclear factor of activated T-cells signaling. Hypertension 51:1010-1019.

Zhou CC, Ahmad S, Mi T, Xia L, Abbasi S, Hewett PW, Sun C, Ahmed A, Kellems RE, and Xia Y (2007) Angiotensin II induces soluble fms-Like tyrosine kinase-1 release via calcineurin signaling pathway in pregnancy. Circ Res 100:88-95.

Zhou CC, Zhang Y, Irani RA, Zhang H, Mi T, Popek EJ, Hicks MJ, Ramin SM, Kellems RE, and Xia Y (2008b) Angiotensin receptor agonistic autoantibodies induce pre-eclampsia in pregnant mice. Nat Med 14:855-862.

Zhu L, Carretero OA, Liao TD, Harding P, Li H, Sumners C, and Yang XP (2010) Role of prolylcarboxypeptidase in angiotensin II type 2 receptor-mediated bradykinin release in mouse coronary artery endothelial cells. Hypertension 56:384-390.

Zhu M, Natarajan R, Nadler JL, Moore JM, Gelband CH, and Sumners C (2000) Angiotensin II increases neuronal delayed rectifier $\mathrm{K}(+)$ current: role of 12-lipoxygenase metabolites of arachidonic acid. J Neurophysiol 84:2494-2501.

Zhu Z, Zhang SH, Wagner C, Kurtz A, Maeda N, Coffman T, and Arendshorst WJ (1998) Angiotensin AT1B receptor mediates calcium signaling in vascular smooth muscle cells of AT1A receptor-deficient mice. Hypertension 31:1171-1177.

Zimmerman B, Beautrait A, Aguila B, Charles R, Escher E, Claing A, Bouvier M, and Laporte SA (2012) Differential $\beta$-arrestin-dependent conformational signaling and cellular responses revealed by angiotensin analogs. Sci Signal 5:ra33.

Zimmerman B, Simaan M, Lee MH, Luttrell LM, and Laporte SA (2009) c-Srcmediated phosphorylation of AP-2 reveals a general mechanism for receptors internalizing through the clathrin pathway. Cell Signal 21:103-110.

Zimpelmann J and Burns KD (2009) Angiotensin-(1-7) activates growth-stimulatory pathways in human mesangial cells. Am J Physiol Renal Physiol 296:F337-F346.

Zohn IE, Symons M, Chrzanowska-Wodnicka M, Westwick JK, and Der CJ (1998) Mas oncogene signaling and transformation require the small GTP-binding protein Rac. Mol Cell Biol 18:1225-1235.

Zou Y, Akazawa H, Qin Y, Sano M, Takano H, Minamino T, Makita N, Iwanaga K, Zhu W, and Kudoh S, et al. (2004) Mechanical stress activates angiotensin II type 1 receptor without the involvement of angiotensin II. Nat Cell Biol 6:499-506. 


\section{Correction to "Angiotensin Receptors: Interpreters of Pathophysiological Angiotensinergic Stimuli"}

In the above article [Karnik S, Unal H, Kemp J, Tirupula K, Eguchi S, Vanderheyden P, and Thomas W (2015) PharmacolRev 67:754-819], the title was incorrect. The correct title is "International Union of Basic and Clinical Pharmacology. XCIX. Angiotensin Receptors: Interpreters of Pathophysiological Angiotensinergic Stimuli". The XHTML and PDF versions of the article have been corrected.

We regret this error and any inconvenience it may have caused. 